\title{
Acoplamento, $\tau$ de Kendall e redução \\ da dimensão de um vetor aleatório
}

\author{
Verónica Andrea González-López
}

- Trabalho de Tese de Doutorado

- Área de concentração Estatística

- Orientador PhD Carlos Alberto de Bragança Pereira

- Orgão financiador CAPES

Instituto de Matemática e Estatística da Universidade de São Paulo Ano 1999 
Acoplamentos e Associação

\section{Capítulo 3 \\ Medidas de Concordância e de Associação}

Seção 3.1: Medidas de Concordância

Seção 3.2: Medida Adequada de Associação

Seção 3.3: Existência do Acoplamento Associado

\section{Capítulo 4}

Análise Exaustiva da Medida $\tau$-Kenda.l

Seção 4.1: Máxima Associação Positiva (Negativa) 


\section{Capítulo 5 \\ Associação e Predição}

Seção 5.1: Construção de um Preditor

Seção 5.2: Adequacidade

183

Seção 5.3: Predição Empírica

197

Apêndice

Seção A.1

203

- Programa 1: Para o Cálculo de Acoplamentos e Medida $\tau$-Kendall

- Programa 2: Simulação

Seção A.2: Condiçio $T P_{2}\left(R R_{2}\right)$ em pares, Distribuição Conjunta e Operação Bayesiana: Prior/Posterior 


\section{Referências}

- Tipos de Dependências

- Acoplamentos e Classes de Fréchet

Indice de Tópicos

Indice de Siglas 


\section{Agradecimentos}

Los caminos que podian ser escogidos para la definición de este trabajo eran muchos, en ese punto comienza el aporte de mi amigo y orientador, profesor Carlinhos, su intuición profunda dió valor a la investigación y estructuración de este "comienzo" el supo inducir mis pasos y motivar mi curiosidad para que optase libremente por cada una de las lineas estudiadas aqui.

Sé que aun estoy en deuda com muchos de los objetivos planteados en el inicio, mas la historia recien comienza...

Paso a paso, ante cada aspecto nuevo que fue necesario estudiar siempre existía un nexo con los trabajos "casi sociales", que mi querido amigo y profesor Sergio recorria, con esa habilidad que solo él consigue, gracias a su aguda percepción del pensamiento humano e intensa adaptación de esas ideas a la formalización matemática. Fue de ese modo que este trabajo terminó adquiriendo forma. Inclusive, agradezco el haber tenido la oportunidad de trabajar junto a Sergio y Luis Gustavo, las discusiones, con ellos entabladas fueron de extrema importancia, mas aun con ellos aprendi a disfrutar de la investigación.

Imprescindibles fueron los aportes y sugerencias de los estimados profesores Jorge y Nelson, que con gran paciencia y dedicación atendieron mis dudas y descubrieron otros aspectos de los resultados aqui presentes. Muchas gracias! 
Agradezco muy especialmente al querido profesor Julio, por su apoyo y excelente humor. Muchas garcias profesor!

Por la parte estética de este trabajo, tanto como sugerencias, me debo al buen censo de Maria Nazareth, Fabio, Marcelo y por supuesto a mi Orientador, quienes permitieron traducir las ideas y motivantes deste trabajo, a ellos, con todo mi afecto! y por su incansable capacidad y actitud ante el trabajo: Muchas gracias!

Entrego este trabajo en manos de "los míos", por quienes vivo... a quienes debo toda la inquietud que me hizo entrar por este camino...

Agradezco, al Instituto de Matemática e Estatística de la Universidade de São Paulo y a CAPES, por brindarme esta oportunidad de desarrollo. 


\section{Resumo}

Considerando um vetor aleatório $n$-variado, $n>2$, com coordenadas Negativamente Associadas (NA), Positivamente Associadas (PA) ou com ambas as condições nele presentes (por exemplo, algumas variáveis aleatórias Associadas em pares e outras variáveis Negativamente Associadas em pares), percorremos as conexões entre os diferentes conceitos. Analisamos e apresentamos opções que tornam uma estrutura "heterogênea" (estrutura mista: positiva e negativa) numa estrutura "homogênea". Em seguida, adotamos uma medida de associação, logo depois de fazer uma análise dessas medidas. Imediatamente, apresentamos as propriedades de tal medida, surgindo. nesse ponto, sua conexão forte com acoplamentos. Essa medida de associação, em seus valores extremos, percorre uma série de considerações feitas por De Finetti (1953), numa proposta para o estudo de campos de coincidência de opiniões. Todos esses resultados, válidos para o caso contínuo. são revisados no caso discreto.

Retomando aquela medida de associação, apresentamos um estudo de uma possível "função preditora". que é intuída a partir da medida e sua invariância ante transformações não decrescentes. A saber, encaramos a teoria de adequacidade, na procura de um ambiente, no qual seja possível fazer uma predição, de "certo modo" razoável, baseados nos dados que contenham toda a informação pertinente a ser usada, para a predição de uma quantidade não observável, em geral; ou, em qualquer forma, uma variável da qual temos informação passada. Nessa predição encontra-se implícita uma redução da dimensão dos dados, observáveis. que leva em conta a estrutura de associação entre seus componentes, através da função de distribuição 
acumulada, do vetor considerado.

Neste trabalho apresentamos uma proposta de metodologia preditiva, baseada nas propriedades das estruturas associadas. Tal metodologia é fundamentada na função de distribuição acumulada obtida a partir do banco de dados ou registros, que possuem estruturas de associação. Para o desenvolvimento da metodologia, apresentamos os fundamentos teóricos das estruturas associadas, acrescentando novos resultados. Apresentamos ainda um método de validação da metodologia proposta, através de resultados que estabelecem uma relação entre as estruturas associadas e a medida $\tau$ de Kendall. 


\section{Abstract}

This monography presents a new methodology for prediction based on the association structure of the random variables envolved. The methodology uses the empirical cumulated distibution function obtained in a previous observed data. The theory needed to develope the method is presented in details and some new results are obtained. To validate the method we present results relating the association structures with the Kendall $\tau$ measure of association. 


\section{Motivação}

O pensamento motivador deste trabalho foi a predição. Os princípios que traçaram os tópicos percorridos baseavam-se na seguinte situação geral: suponhamos que temos acesso a um banco de dados, constituído por realizações de um vetor aleatório $\left(X_{1}, X_{2}, \cdots, X_{n}\right)$, onde parte dele é observada $\left(X_{2}, \cdots, X_{n}\right)$, enquanto que $X_{1}$ é uma variável aleatória derivada a partir do conhecimento de $\left(X_{2}, \cdots, X_{n}\right)$. Logo, o banco de dados à nossa disposição é : $\left\{\left(x_{i 1}, x_{i 2}, \cdots, x_{i n}\right)\right\}_{i=1}^{N-1}$. Suponhamos agora que um novo vetor, $\left(x_{N 2}, \cdots, x_{N n}\right)$, é observado; Qual seria uma predição adequada para $x_{N 1}$ ?. A saber, pretendemos dar um tratamento para aquele banco de dados, que seja induzido pelo conhecimento da estrutura de associação das variáveis envolvidas. Este problema, muito geral, levou-nos ao desenvolvimento de aspectos diversos, de maneira a dar definição e contexto para o tratamento do mesmo.

Foi preciso escolher uma linha de raciocínio, montar um esquema fornecedor do ambiente no qual pretendíamos enquadrar o problema. O Capítulo 1 discorre sobre conceitos ante os quais, "acreditamos", nosso problema responde. Dentre as razões para essa escolha temos: como a variável $X_{1}$ é resultante a partir de $\left(X_{2}, \cdots, X_{n}\right)$, admitimos então que exite uma forte dependência entre $X_{1}$ e cada uma das outras, tanto quanto $X_{1}$ depende do vetor $\left(X_{2}, \cdots, X_{n}\right)$ como um todo. Nesse mesmo Capítulo fica evidente a dificuldade perante situações de estruturas aleatórias mistas (dependências positivas e negativas), pois tais estruturas correspondem a variáveis aleatórias concordantes e discordantes, respectivamente. No Capítulo 2 apresentamos soluções alternativas para estruturas mistas. Outro aporte feito nesse Capítulo refere-se à possibilidade de reconstruir alguns resultados já conhecidos para 
o caso de positividade, na presença de negatividade.

A idéia que governou a construção do preditor - que assumimos unidimensional, pois a variável a predizer $X_{1}$ é unidimensional - orientou nossos passos ao estudo de medidas de associação, surgidas a partir das medidas de concordância. Intuitivamente, aquele preditor devia potencializar a associação entre $X_{1}$ e cada variável da base observacional, $\left(X_{2}, \cdots, X_{n}\right)$. Mais tarde, essa intuição levou nosso estudo até a medida $\tau$ de Kendall e os acoplamentos associados (distribuições de marginais uniformes).

Continuando nessa linha, o Capítulo 4 trata especificamente os casos de máxima associação: por intermédio da medida $\tau$-Kendall é possivel enxergar a relação entre as variáveis medidas por $\tau$. Foi implementado um programa para o cálculo da medida $\tau$ tanto quanto para o cálculo dos acoplamentos, em casos bivariados discretos adicionado no apêndice do trabalho.

Finalmente, no Capítulo 5 fazemos um estudo analítico da estrutura do preditor de $X_{1}$, colocando em evidência diversos aspectos que o caracterizam. Este preditor será gerado levando-se em conta a informação contida na distribuição acumulada do vetor $\left(X_{2}, \cdots, X_{n}\right)$.

Nesta mesma direção, apresentamos os conceitos de adequacidade. Com base nos princípios que estruturam nosso preditor, apresentamos propostas para uma predição adequada. 
A predição empírica, discutida ao final do Capítulo, analisa a manipulação do banco de dados, assim como o uso de toda a "informação" contida na amostra informação esta embutida na distribuição acumulada empírica. 
"Este palacio es fabrica de los dioses, pensé primeramente. Exploré los inhabitados recintos y corregí: Los dioses que lo edificaron han muerto. Noté sus peculiaridades y dije: Los dioses que lo edificaron estaban locos. Lo dije, bien lo sé, con una incomprensible reprovación que era casi un remordimiento...

Pensé que Argos y yo participábamos de universos distintos; pensé que nuestras percepciones eran iguales, pero que Argos las combinaba de otra manera y construía con ellas otros objetos; pensé que acaso no había objetos para él, sino un vertiginoso y continuo juego de impresiones brevísimas. Pensé en un mundo sin memoria. sin tiempo..."

Jorge Luis Borges (1974), El inmortal-El Aleph.

\section{Capítulo 1 \\ Conceitos de Dependência}

- Seção 1.1: Introdução e Contexto

- Seção 1.2: Definições

- Seção 1.3: Resultados Essenciais, que Caracterizam os Diferentes Tipos de Dependências e suas Conexões

- Seção 1.4: Exemplos 


\subsection{Introdução e contexto}

Nos trabalhos de Joag-Dev e Proschan (1983) são apresentados diversos exemplos de vetores aleatórios $N A$ e com f.d.p. $S-M R R_{2}$ (densidade "estritamente regra reversa de ordem 2", como as distribuições (a): Multinomial, (b): Hipergeométrica Multivariada, (c): Dirichlet, (d): Dirichlet composta com a Multinomial e (e): Medidas invariantes por permutação. Outros enfoques são abordados por Block et al.(1982) apresentando (a), (b), (c), (d) e (f): Normal Multivariada com estrutura especial de covariância como exemplos de f.d.p. $R R_{2}$ em pares (densidade regra reversa de ordem 2, em pares) e, conseqüentemente, $S-M R R_{2}$. No entanto, Karlin e Rinott (1980 b) exibiram provas elaboradas de (a), (b), (c), (f) e (g): distribuição Hahn Multivariada, como densidades $S-M R R_{2}$.

Karlin e Rinott (1980 a) também expõem numerosos exemplos de densidades $M T P_{2}$ ("multivariada totalmente positiva de ordem 2") que envolvem as multinormais (por exemplo, $\underline{X} \sim N(\underline{0}, \Sigma)$, tal que os elementos fora da diagonal de $-\Sigma^{-1}$ sejam não negativos) e ainda provam que variáveis aleatórias independentes possuem f.d.p. conjunta $M T P_{2}$. Outros exemplos de funções de distribuição $M T P_{2}$ são, (i): Logística Multivariada, (ii): Gama Multivariada, (iii): F-Multivariada, (iv): Valor Absoluto Cauchy Multivariada, (v): Multinomial Negativa.

Em 1997, Joe fornece um resultado que mostra a conexão existente, no caso bivariado, entre f.d.p. $T P_{2}$ ("totalmente positiva de ordem 2", bivariada) e variáveis aleatórias $P A$ (f.d.p. $T P_{2}$ implica em variáveis $P A$ ). No entanto, Joag-Dev e Proschan (1983) já tinham provado para o caso bivariado que f.d.p. $R R_{2}$ implica 
em variáveis aleatórias $N A$.

Considerando um vetor aleatório $n$-variado, $n>2$, com coordenadas Negativamente Associadas, Positivamente Associadas ou com ambas as condições presentes (por exemplo, algumas variáveis aleatórias Associadas em pares e outras variáveis Negativamente Associadas em pares) e um componente, que designaremos como variável a predizer, enquanto que, as $n-1$ componentes restantes denotaremos como variáveis base da predição. Pretendemos mostrar que é conveniente utilizar para a criação de um preditor, fundamentado na base, "toda a informação" contida na distribuição acumulada que vincula todo o vetor aleatório (variáveis observáveis e variável a predizer). Por exemplo, consideremos que $\underline{U}=\left(U_{1}, U_{2}, U_{3}\right)$ é o vetor aleatório, onde as variáveis envolvidas têm "marcada dependência entre si". Suponhamos que $U_{2}$ e $U_{3}$ são resultados de análises clínicas e $U_{1}$ é o diagnóstico elaborado na base das outras duas. A associação existente entre $U_{1}$ e $U_{i}, i=2,3$ medida através da medida bivariada $\tau$ de Kendall, será "pronunciada" por um preditor, que condense a informação embutida na distibuição conjunta de $\underline{U}$.

A situação anterior exige um detalhado estudo das possíveis dependências entre variáveis aleatórias, suas relações e "história bibliográfica". Neste ponto, apresentamos algumas humildes contribuições, que permitiram enxergar com maior detalhe o alcance e interpretação dos conceitos. Primeiramente, surgiu a preocupação por estruturas combinadas (algumas variáveis positivamente associadas e outras negativamente associadas). Diante disso. estabelecemos condições sobre o vetor que permitiram obter um novo vetor (transformação do anterior) de coordenadas $P A$ em pares ou $N A$ em pares, substituindo a estrutura de associação heterogênea por 
uma estrutura de associação homogênea.

Perante aquela preocupação toda em relação à associação entre variáveis, fizemos uma moderada pesquisa entre as medidas de concordância, optando pela medida $\tau$ de Kendall, como a mais adaptada ao nosso contexto. No caminho, tentamos motivar a intuição com exemplos ilustrativos. Em seguida, estudamos o comportamento da medida bivariada $\tau$ de Kendall em casos contínuos e discretos.

Percorremos os valores extremos fornecidos pela medida $\tau$ na procura do caráter informativo, em sua relação com a distribuição conjunta e a relação "tipo funcional" existente entre as variáveis para as quais a medida é extrema. 


\subsection{Definições e Notações Gerais}

Daremos, a seguir, as definições básicas que necessitaremos:

$$
\begin{aligned}
& \text { Se } \underline{X}=\left(X_{1}, \cdots, X_{n}\right) \text { e } \underline{Y}=\left(Y_{1}, \cdots, Y_{n}\right), \text { denotamos por } \\
& \underline{X} \vee \underline{Y}:=\left(\max \left\{X_{1}, Y_{1}\right\}, \cdots, \max \left\{X_{n}, Y_{n}\right\}\right), \\
& \underline{X} \wedge \underline{Y}:=\left(\min \left\{X_{1}, Y_{1}\right\}, \cdots, \min \left\{X_{n}, Y_{n}\right\}\right) .
\end{aligned}
$$

\subsubsection{Definição}

Dizemos que o vetor aleatório $\underline{X}=\left(X_{1}, \cdots, X_{n}\right)$ é

(i) Negativamente Associado (NA) [Proschan e Joag-Dev (1983)] se, para toda partição de $\underline{X}$ em $\underline{X}_{1}=\left(X_{\pi(1)}, \cdots, X_{\pi(k)}\right)$ e $\underline{X}_{2}=\left(X_{\pi(k+1)}, \cdots, X_{\pi(n)}\right)$ e para todo par de funções crescentes $f$ e $g$ sobre $R^{k}$ e $R^{n-k}$, respectivamente, temos

$$
\operatorname{Cov}\left(f\left(\underline{X}_{1}\right), g\left(\underline{X}_{2}\right)\right) \leq 0
$$

onde $\pi$ é uma permutação arbitrária de $\{1, \cdots, n\}$.

(ii) Negativamente Associado (NA) em pares, se a definição anterior se verifica para todo par de coordenadas do vetor $\underline{X}$. 


\subsubsection{Definição}

Dizemos que o vetor aleatório $\underline{X}=\left(X_{1}, \cdots, X_{n}\right)$ é

(i) Positivamente Associado (PA ou A) [Joag-Dev (1983)] (ou as variáveis aleatórias $X_{1}, \cdots, X_{n}$ são $\left.P A\right)$ se, para todo par de funções crescentes (em cada coordenada) $f$ e $g$ sobre $R^{n}$, temos $\operatorname{Cov}(f(\underline{X}), g(\underline{X})) \geq 0$.

(ii) Positivamente Associado (PA ou $A$ ) em pares se, a definição anterior se verifica para todo par de coordenadas do vetor $\underline{X}$.

1.2.3 Definição [Karlin e Rinott (1980) a, b ]

Seja $f: R^{n} \rightarrow R$ uma função não negativa,

(i) dizemos que $f$ é $M T P_{2}$ (Multivariate totally positive of order 2) se,

$$
f(\underline{X} \vee \underline{Y}) f(\underline{X} \wedge \underline{Y}) \geq f(\underline{X}) f(\underline{Y})
$$

$\forall \underline{X}, \underline{Y}$ vetores em $R^{n}$

(ii) Dizemos que $f$ é $M R R_{2}$ (Multivariate reverse rule of order 2) se,

$$
f(\underline{X} \vee \underline{Y}) f(\underline{X} \wedge \underline{Y}) \leq f(\underline{X}) f(\underline{Y})
$$

$\forall \underline{X}, \underline{Y}$ vetores em $R^{n}$ 
1.2.4 Definição [Karlin e Rinott (1980) a, Efron (1964)]

Uma função $\xi: R \rightarrow R$ não negativa é dita $P F_{2}$ (freqüência polya de ordem 2) se a transformação $f: R^{2} \rightarrow R$ definida por

$$
f\left(x_{1}, y_{1}\right):=\xi\left(x_{1}-y_{1}\right) \text { é } T P_{2}
$$

onde, $T P_{2}$ (Totally positive of order 2) corresponde à definição de $M T P_{2}$ no caso bivariado.

Nota: análogamente denotamos por $R R_{2}$ (Reverse rule of order 2) quando, temos uma função bivariada $M R R_{2}$.

1.2.5 Definição [Block, Savits, Shaked (1982)]

Seja $f: R^{n} \rightarrow R$ uma função não negativa.

(i) Dizemos que $f$ é $R R_{2}$ em pares se $f$ é $R R_{2}$ fixando-se $n-2$ coordenadas arbitrárias.

(ii) Dizemos que $f$ é $T P_{2}$ em pares se $f$ é $T P_{2}$ fixando-se $n-2$ coordenadas arbitrárias. 
1.2.6 Definição [Karlin e Rinott (1980) b]

Uma função $M R R_{2}, f: R^{n} \rightarrow R$ é fortemente $M R R_{2}$, " $S-M R R_{2}$ " se para qualquer conjunto de $n$ funções $P F_{2},\left\{\xi_{i}\right\}_{i=1}^{n}$, e para cada $j \leq n$, a função:

$$
g_{k-j}\left(x_{i_{j+1}}, \cdots, x_{i_{n}}\right):=\int \cdots \int f(\underline{x}) \prod_{m=1}^{j} \xi_{m}\left(x_{i_{m}}\right) d x_{i_{m}}
$$

é $M R R_{2}$, sempre que a integral exista, onde, $\left\{i_{1}, \cdots, i_{n}\right\}$ é qualquer permutação de $\{1, \cdots, n\}$.

1.2.7 Definição [Karlin e Rinott (1980) a]

Sejam $f_{1}$ e $f_{2}$ duas funções de distribuições de probabilidade em $R^{n}$. Se para todo $\underline{x} \in R^{n}, \underline{y} \in R^{n}$

$$
f_{2}(\underline{x} \vee \underline{y}) f_{1}(\underline{x} \wedge \underline{y}) \geq f_{1}(\underline{x}) f_{2}(\underline{y})
$$

dizemos que, $f_{2}>_{T P 2} f_{1}$.

1.2.8 Definição [Karlin e Rinott (1980) a, b]

Seja $\underline{X}=\left(X_{1}, \cdots, X_{n}\right)$ um vetor aleatório com função de densidade $f_{\underline{X}}$.

Se $f_{\underline{X}}$ é $M T P_{2}\left(M R R_{2}\right)$ dizemos que $\underline{X}$ é $M T P_{2}\left(M R R_{2}\right)$ ou que $X_{1}, \cdots, X_{n}$ são $M T P_{2}\left(M R R_{2}\right)$. 
1.2.9 Definição [Proschan e Joag Dev (1983)]

Sejam $J, K$ intervalos fechados à direita e abertos pela esquerda em $R$. Para variáveis aleatórias $X$ e $Y$ definimos

$$
H(J, K):=P(X \in J, Y \in K)
$$

dizemos que $(X, Y)$ é $T P_{2}\left(R R_{2}\right)$ em intervalos se para $J_{1}<J_{2}, K_{1}<K_{2}$,

$$
H\left(J_{2}, K_{2}\right) H\left(J_{1}, K_{1}\right) \geq(\leq) H\left(J_{1}, K_{2}\right) H\left(J_{2}, K_{1}\right)
$$

onde $I_{1}<I_{2}$ denota, $x \leq y \forall x \in I_{1} . \forall y \in I_{2}, I_{i}$, intervalo em $R, i=1,2$.

\subsubsection{Definição [Lehmann (1966)]}

Sejam $X$ e $Y$ duas variáveis aleatórias, dizemos que elas são $N Q D(P Q D)$, i.e., Negative quadrant dependent (Positive quadrant dependent) se para todo par de valores reais $(x, y)$, verifica-se,

$$
P(X \leq x, Y \leq y) \leq(\geq) P(X \leq x) P(Y \leq y)
$$

1.2.11 Definição [Karlin e Rinott (1980) a, b]

Sejam $X_{1}, X_{2}, \cdots, X_{n}$, variáveis aleatórias. Dizemos que são $N U O D(P U O D)$ i.e., Negative upper orthant dependence (Positive upper orthant dependence), se para toda $n$-upla real $\left(x_{1}, x_{2}, \cdots, x_{n}\right)$, temos

$$
P\left(X_{i}>x_{i}, i=1, \cdots, n\right) \leq(\geq) \prod_{i=1}^{n} P\left(X_{i}>x_{i}\right) .
$$


E dizemos que são NLOD (PLOD), i.e., Negative lower orthant dependence (Positive lower orthant dependence), se para toda $n$-upla real $\left(x_{1}, x_{2}, \cdots, x_{n}\right)$, verifica-se,

$$
P\left(X_{i} \leq x_{i}, i=1, \cdots, n\right) \leq(\geq) \prod_{i=1}^{n} P\left(X_{i} \leq x_{i}\right)
$$

1.2.12 Definição [Karlin e Rinott (1980) a, b]

(i) Dizemos que variáveis aleatórias são NOD (Negative orthant dependence) se são $N U O D$ e $N L O D$.

(ii) Dizemos que variáveis aleatórias são $P O D$ (Positive orthant dependence) se são $P U O D$ e $P L O D$.

Notemos que para $n=2$,

$$
N O D \Leftrightarrow N Q D \text { e } P O D \Leftrightarrow P Q D .
$$

\subsubsection{Definição [Joe (1997)]}

Um vetor aleatório $\underline{X}=\left(X_{1}, X_{2}\right)$ com função de distribuição acumulada $F$ é "Estocasticamente decrescente (crescente)" em $X_{1}$, ou a distribuição condicional $F_{2 \mid 1}$ é "Estocasticamente decrescente (crescente)", se

$$
P\left(X_{2}>x_{2} \mid X_{1}=x_{1}\right)=1-F_{2 \mid 1}\left(x_{2} \mid x_{1}\right) \downarrow(\uparrow) x_{1} \forall x_{2},
$$

i.e.,

$$
F_{2 \mid 1}\left(x_{2} \mid x_{1}\right) \uparrow(\downarrow) \text { em } x_{1} \forall x_{2}
$$


onde $F_{2 \mid 1}\left(x_{2} \mid x_{1}\right)=P\left(X_{2} \leq x_{2} \mid X_{1}=x_{1}\right)$. Usaremos a notação: $X_{2} S D(S I) X_{1}$.

Nota: excepto especificações prévias, é assumida a seguinte notação

Se $\underline{X}=\left(X_{1}, \cdots, X_{n}\right)$ é um vetor aleatório, denotamos por $F_{\underline{X}}$ e $f_{\underline{X}}$ as respectivas f.d.a. (função de distribuição acumulada) e f.d.p. (função de densidade de probabilidade) conjuntas. Entanto que as marginais univariadas respectivas serão chamadas $F_{X_{i}}$ e $f_{X_{i}}, i=1, \cdots, n$.

\subsubsection{Definição [Joe (1997)]}

Seja $\underline{X}=\left(X_{1}, \cdots, X_{n}\right)$ um vetor aleatório com f.d.a. F. Dizemos que, $\underline{X}$ é "Positivamente dependente através da ordem estocástica" $(P D S)$ se a distribuição condicional de $\left\{X_{i}: i \neq j\right\}$ dado $X_{j}=x$ é Estocasticamente crescente em $x$ para todo $j=1, \cdots, n$.

\subsubsection{Definição [Joe (1997)]}

Seja $\underline{X}=\left(X_{1}, \cdots, X_{n}\right)$ um vetor aleatório com f.d.a. $F$. Dizemos que, $\underline{X}$ é "Negativamente dependente através da ordem estocástica" (NDS) se a distribuição condicional de $\left\{X_{i}: i \neq j\right\}$ dado $X_{j}=x$ é Estocasticamente decrescente em $x$ para todo $j=1, \cdots, n$. 


\subsubsection{Definição [Joe (1997)]}

Seja $\underline{X}=\left(X_{1}, \cdots, X_{n}\right)$ um vetor aleatório. Dizemos que $\underline{X}$ é "Condicionalmente decrescente (crescente) em seqüência", denotado por $C D S(C I S)$, se $X_{i}$ é Estocasticamente decrescente (crescente) em $X_{1}, \cdots, X_{i-1}$ para $i=2, \cdots, n$. i.e.,

$$
P\left(X_{i}>x_{i} \mid X_{j}=x_{j}, j=1, \cdots, i-1\right) \downarrow(\uparrow) x_{1}, \cdots, x_{i-1} \forall x_{i} .
$$

\subsubsection{Definição [Joe (1997)]}

Seja $\underline{X}=\left(X_{1}, X_{2}\right)$ um vetor aleatório com f.d.a. $F$ e com marginais $F_{i}, i=1,2$. Dizemos que, $X_{2}$ é "Decrescente (Crescente) pela cauda direita em $X_{1}$ ", se

$$
P\left(X_{2}>x_{2} \mid X_{1}>x_{1}\right)=\frac{\bar{F}\left(x_{1}, x_{2}\right)}{\bar{F}_{1}\left(x_{1}\right)} \downarrow(\uparrow) x_{1} \forall x_{2},
$$

onde $\bar{F}\left(x_{1}, x_{2}\right)=P\left(X_{1}>x_{1}, X_{2}>x_{2}\right)$. Notação: $X_{2} \operatorname{RTD} X_{1}\left(X_{2} \operatorname{RTI} X_{1}\right)$.

\subsubsection{Definição [Joe (1997)]}

Seja $\underline{X}=\left(X_{1}, X_{2}\right)$ um vetor aleatório com f.d.a. $F$ e com marginais $F_{i}, i=1,2$. Dizemos que, $X_{2}$ é "Crescente (Decrescente) pela cauda esquerda em $X_{1}$ ", se

$$
P\left(X_{2} \leq x_{2} \mid X_{1} \leq x_{1}\right)=\frac{F\left(x_{1}, x_{2}\right)}{F_{1}\left(x_{1}\right)} \uparrow(\downarrow) x_{1} \forall x_{2}
$$

Notação: $X_{2} \operatorname{LTI} X_{1}\left(X_{2} \operatorname{LTD} X_{1}\right)$.

\footnotetext{
${ }^{0} \mathrm{NO}$ desenvolvimento da tese - salvo especificação prévia - assumiremos como ordem em $R^{n}$. a ordem parcial usual, isto é: $\left\{X_{k} \leq x_{k}, k \in\left\{i_{1}, \cdots, i_{s}\right\}\right\}$ expressa que para todo $k X_{k} \leq x_{k}, k \in$ $\left\{i_{1}, \cdots, i_{s}\right\}$.
} 
1.2.19 Definição [Joe (1997)]

Seja $\underline{X}=\left(X_{1}, \cdots, X_{n}\right)$ um vetor aleatório com f.d.a. F. $X_{i}$ é $R T D(R T I) \mathrm{em}$ $X_{j}, i \in A^{c}, j \in A$, se

$$
P\left(X_{i}>x_{i}, i \in A^{c} \mid X_{j}>x_{j}, j \in A\right) \downarrow(\uparrow) x_{k}, k \in A,
$$

onde, $A$ é um subconjunto não vazio de $\{1, \cdots, n\}$.

1.2.20 Definição [Joe (1997)]

Seja $\underline{X}=\left(X_{1}, \cdots, X_{n}\right)$ um vetor aleatório com f.d.a. F. $X_{i}$ é $L T I(L T D)$ em $X_{j}, i \in A^{c}, j \in A$, se

$$
P\left(X_{i} \leq x_{i}, i \in A^{c} \mid X_{j} \leq x_{j}, j \in A\right) \uparrow(\downarrow) x_{k}, k \in A,
$$

onde, $A$ é um subconjunto não vazio de $\{1, \cdots, n\}$.

\subsubsection{Fórmula da Composição [Karlin e Rinott(1968)]}

Consideremos uma função $K: R^{2} \rightarrow R$, definamos o operador $K^{*}: R^{2 m} \rightarrow R$

$$
K^{*}\left(\begin{array}{c}
x_{1}, x_{2}, \cdots, x_{m} \\
y_{1}, y_{2}, \cdots, y_{m}
\end{array}\right):=\left|\begin{array}{cc}
K\left(x_{1}, y_{1}\right) & K\left(x_{1}, y_{2}\right) \cdots K\left(x_{1}, y_{m}\right) \\
K\left(x_{2}, y_{1}\right) & K\left(x_{2}, y_{2}\right) \cdots K\left(x_{2}, y_{m}\right) \\
\vdots & \vdots \\
K\left(x_{m}, y_{1}\right) & K\left(x_{m}, y_{2}\right) \cdots K\left(x_{m}, y_{m}\right)
\end{array}\right|
$$

onde $x_{i} \leq x_{i+1}, y_{i} \leq y_{i+1}, i=1, \cdots, m-1$. 
Uma função $K$ é "Sinal-Regular de ordem $r$ " $\left(S R_{r}\right)$ se existir uma seqüência de números $\left\{\epsilon_{m}\right\}_{m}$, onde $\epsilon_{m}$ pode ser +1 ou -1 , tal que

$$
\epsilon_{m} K^{*}\left(\begin{array}{c}
x_{1}, x_{2}, \cdots, x_{m} \\
y_{1}, y_{2}, \cdots, y_{m}
\end{array}\right) \geq 0, \quad 1 \leq m \leq r .
$$

O símbolo $\epsilon_{m}(K)=\epsilon_{m}$ denota o sinal (constante) do determinante da $m$-ésima ordem.

Se $\epsilon_{m}=(-1)^{m(m-1) / 2}$, dizemos que $K$ é "Sinal-regra reversa de ordem $r$ " $\left(R R_{r}\right)$.

Sejam $K, L, M$ funções borel-mensuráveis de duas variáveis satisfazendo,

$$
M(\xi, \eta)=\int_{Y} K(\xi, \zeta) L(\zeta, \eta) d \sigma(\zeta) \quad \xi \in X, \eta \in Z
$$

Assumindo que a integral é absolutamente convergente; $X, Y, Z$ subconjuntos de $R, d \sigma(\zeta)$ uma medida $\sigma$-finita definida sobre $Y$.

$$
\begin{aligned}
& M^{*}\left(\begin{array}{c}
\xi_{1}, \cdots, \xi_{m} \\
\eta_{1}, \cdots, \eta_{m}
\end{array}\right) \text { é dado pela seguinte } \\
& =\frac{1}{m !} \quad \begin{array}{c}
\int \cdots \int \\
\zeta_{1}<\cdots \zeta_{m}, \zeta_{i} \in Y
\end{array} K^{*}\left(\begin{array}{c}
\xi_{1}, \cdots, \xi_{m} \\
\zeta_{1}, \cdots, \zeta_{m}
\end{array}\right) L^{*}\left(\begin{array}{c}
\zeta_{1}, \cdots, \zeta_{m} \\
\eta_{1}, \cdots, \eta_{m}
\end{array}\right) d \sigma\left(\zeta_{1}\right) \cdots d \sigma\left(\zeta_{m}\right)
\end{aligned}
$$

Vide [Karlin(1968)pp.99] Lema 1.1.

(a) Se $K(\xi, \zeta)$ é $S R_{r}$ e $L(\zeta, \eta)$ é $S R_{s}$, então, $M(\xi, \eta)$ é $S R_{\min \{r, s\}}$ e $\epsilon_{p}(M)=$ $\epsilon_{p}(K) \epsilon_{p}(L), p=1, \cdots, \min \{r, s\}$. 
1.2.22 Definição Acoplamento [Sklar (1959), Joe(1997)]

Acoplamento é uma distribuição multivariada com todas suas marginais $U(0,1)$.

Para um vetor aleatório $\underline{X}=\left(X_{1}, \cdots, X_{n}\right)$ com f.d.a. $F_{\underline{X}}$ e com marginais $F_{X_{1}}, \cdots, F_{X_{n}}$. Um acoplamento associado a $F_{\underline{X}}$ é uma função de distribuição acumulada $C_{\underline{X}}: I^{n} \rightarrow I, I=[0,1]$, de marginais uniformes em $(0,1)$ e que satisfaz,

$$
F_{\underline{X}}(\underline{x})=C_{\underline{X}}\left(F_{X_{1}}\left(x_{1}\right), \cdots, F_{X_{n}}\left(x_{n}\right)\right), \quad \forall \underline{x} \in R^{n}, \underline{x}=\left(x_{1}, \cdots, x_{n}\right) .
$$

1.2.23 Definição Medida $\tau$ de Kendall [Nelsen (1992), Joe (1997)]

Sejam $\left(X_{1}^{\prime}, X_{2}^{\prime}\right)$ e $\left(X_{1}^{\prime \prime}, X_{2}^{\prime \prime}\right)$ dois pares de vetores aleatórios, ambos com f.d.a. $F_{X_{1}, X_{2}}$. Os pares são realizações do vetor $\left(X_{1}, X_{2}\right)$. Definimos a medida $\tau_{X_{1}, X_{2}}(\tau$ de Kendall), entre as variáveis $X_{1}$ e $X_{2}$ por

$$
\tau_{X_{1}, X_{2}}:=P\left\{\left(X_{1}^{\prime}-X_{1}^{\prime \prime}\right)\left(X_{2}^{\prime}-X_{2}^{\prime \prime}\right) \geq 0\right\}-P\left\{\left(X_{1}^{\prime}-X_{1}^{\prime \prime}\right)\left(X_{2}^{\prime}-X_{2}^{\prime \prime}\right)<0\right\} .
$$

\footnotetext{
${ }^{0}$ Os resultados indicados com * são propostas e interpretações próprias deste trabalho. Alguns deles são variações de resultados já existentes na literatura que são devidamente especificados, quando for o caso.
} 


\subsection{Resultados essenciais, que caracterizam dife- rentes tipos de dependências e suas conexões}

Apresentamos a seguir um compêndio de resultados que caracterizam os diferentes tipos de dependências. Todos eles estão direcionados, basicamente, para o estudo dos conceitos de variáveis positivamente (negativamente) associadas e $M T P_{2}\left(M R R_{2}\right)$.

A partir desta síntese é possível compreender que os conceitos de positividade e negatividade (nos termos expostos até agora) em geral, não são opostos. Além disso a diversidade das "boas" propriedades da positividade, nem sempre é preservada na sua versão negativa.

Os resultados aqui citados são demonstrados de maneira detalhada nos artigos referenciados.

\section{Positividade}

1.3.1 Teorema [Teorema 2.1., Karlin e Rinott(1980a)] Sejam $f_{1}, f_{2}, f_{3}, f_{4}$, funções não negativas sobre $R^{n}$, satisfazendo.

$$
f_{1}(\underline{x}) f_{2}(\underline{y}) \leq f_{3}(\underline{x} \vee \underline{y}) f_{4}(\underline{x} \wedge \underline{y})
$$

para todo $\underline{x}, \underline{y} \in R^{n}$. Então,

$$
\int_{-\infty}^{+\infty} f_{1}(\underline{x}) d \underline{x} \int_{-\infty}^{+\infty} f_{2}(\underline{x}) d \underline{x} \leq \int_{-\infty}^{+\infty} f_{3}(\underline{x}) d \underline{x} \int_{-\infty}^{+\infty} f_{4}(\underline{x}) d \underline{x} .
$$


O Teorema anterior possibilita a manipulação dos tipos de dependência $T P_{2}$ tanto como a ordem $>_{T P_{2}}$.

1.3.2 Teorema [Corolário 4.15., Barlow e Proschan(1981)] Se $\left(X_{1}, \cdots, X_{n}\right)$ tem f.d.p.TP $P_{2}$ em pares, então, $\left\{X_{1}, \cdots, X_{n}\right\}$ são $P A$.

Caracterizando a classe das funções $M T P_{2}$

1.3.3 Proposição [Proposição 2.1., Karlin e Rinott(1980a)] Seja $f(\underline{x}) T P_{2}$ em pares para todo $\underline{x} \in R^{n}$, e suponha que $f(\underline{x}) f(\underline{y}) \neq 0$ implica $f(\underline{u}) f(\underline{v}) \neq 0 \forall \underline{x}, \underline{y} \in$ $R^{n}, \underline{x} \wedge \underline{y} \leq \underline{u}, \underline{v} \leq \underline{x} \vee \underline{y}$. Então,

$$
f(\underline{x}) f(\underline{y}) \leq f(\underline{x} \vee \underline{y}) f(\underline{x} \wedge \underline{y}), \forall \underline{x}: \underline{y} \in R^{n} .
$$

1.3.4 Proposição [Proposição 3.6., Karlin e Rinott(1980a)] Se $f(\underline{x})$ é $M T P_{2}, \underline{x} \in R^{n}$ e $\left\{\varphi_{i}\right\}_{i=1}^{n}$ é uma seqüência de funções monótonas concordantes, sobre $R$. Então

$$
\psi(\underline{x}):=f\left(\varphi_{1}\left(x_{1}\right), \cdots, \varphi_{n}\left(x_{n}\right)\right)
$$

é $M T P_{2}$ em $R^{n}$.

1.3.5 Proposição [Proposição 3.3., Karlin e Rinott(1980a)] Se $f$ e $g$ são funções $M T P_{2}$. Então, $f g$ é $M T P_{2}$.

1.3.6 Proposição [Proposição 3.5., Karlin e Rinott(1980a)] Variáveis aleatórias independentes têm densidade conjunta $M T P_{2}$.

1.3.5 implica 1.3.6. 
1.3.7 Proposição [Proposição 3.1., Karlin e Rinott(1980a)] Sejam $f_{1}, f_{2}, f_{3}, f_{4}$, funções não negativas sobre $R^{n}$, satisfazendo,

$$
f_{1}(\underline{x}) f_{2}(\underline{y}) \leq f_{3}(\underline{x} \vee \underline{y}) f_{4}(\underline{x} \wedge \underline{y})
$$

para todo $\underline{x}, \underline{y} \in R^{n}$. Então $\forall \underline{x}, \underline{y} \in R^{k}$, tem-se,

$$
\varphi_{1}(\underline{x}) \varphi_{2}(\underline{y}) \leq \varphi_{3}(\underline{x} \vee \underline{y}) \varphi_{4}(\underline{x} \wedge \underline{y})
$$

onde,

$$
\varphi_{i}\left(x_{j_{1}}, \cdots, x_{j_{k}}\right):=\int_{R} \cdots \int_{R} f_{i}(\underline{x}) d x_{j_{k+1}} \cdots d x_{j_{n}}, i=1, \cdots, 4,
$$

onde $j_{1} \leq \cdots \leq j_{k}, k<n$, e o conjunto de índices complementares de $\left\{j_{1}, \cdots, j_{k}\right\}$ (respeito de $\{1, \cdots, n\})$ é: $\left\{j_{k+1}, \cdots, j_{n}\right\}$

\subsection{1 implica 1.3.7}

1.3.8 Proposição [Proposição 3.2., Karlin e Rinott(1980a)] Seja $f$ uma função $M T P_{2}$ sobre $R^{n}$. Então, a função marginal $\varphi$ definida sobre $R^{k}$ por

$$
\varphi\left(x_{1}, \cdots, x_{k}\right):=\int_{R} \cdots \int_{R} f\left(x_{1}, \cdots, x_{k}, x_{k+1}, \cdots, x_{n}\right) d x_{k+1} \cdots d x_{n},
$$

é $M T P_{2}$.

1.3.7 implica 1.3.8.

1.3.9 Proposição [Proposição 3.7., Karlin e Rinott(1980a)] Seja $\underline{X}=\left(X_{1}, \cdots, X_{n}\right)$ um vetor aleatório de componentes independentes, com densidades marginais $f_{X_{1}}, P F_{2}$. Seja $\underline{Y}=\left(Y_{1}, \cdots, Y_{n}\right)$ um vetor aleatório com densidade conjunta $M T P_{2}$ sobre $R^{n}$, e suponha que $\underline{X}$ e $\underline{Y}$ são independentes. Então, $\underline{X}+\underline{Y}$ tem densidade $M T P_{2}$. 
1.3.8 implica 1.3.9.

1.3.10 Proposição [Proposição 3.11., Karlin e Rinott (1980) a] Sejam $X_{1}, \cdots, X_{n}$ variáveis aleatórias i.i.d. cada uma dela com densidade $f$. Então, a densidade conjunta das estatísticas de ordem $X_{(1)}, \cdots, X_{(n)}$ é $M T P_{2}$.

\section{Negatividade}

Caracterizando a classe das funções $M R R_{2}$

1.3.11 Proposição [Proposição, Karlin e Rinott(1980b)] Seja $f(\underline{x}) R R_{2}$ em pares para todo $\underline{x} \in R^{n}$, e suponha que $f(\underline{u}) f(\underline{v}) \neq 0$ implica em $f(\underline{x}) \neq 0 \forall \underline{u}, \underline{v} \in$ $R^{n}, \underline{u} \leq \underline{x} \leq \underline{v}$. Então,

$$
f(\underline{x}) f(\underline{y}) \geq f(\underline{x} \vee \underline{y}) f(\underline{x} \wedge \underline{y}), \forall \underline{x}, \underline{y} \in R^{n}
$$

1.3.12 Proposição [Proposição, Karlin e Rinott(1980b)] Se $f(\underline{x})$ é $M R R_{2}, \underline{x} \in R^{n}$ e $\left\{\varphi_{i}\right\}_{i=1}^{n}$ é uma sequência de funções monótonas concordantes, sobre $R$. Então,

$$
\psi(\underline{x}):=f\left(\varphi_{1}\left(x_{1}\right), \cdots, \varphi_{n}\left(x_{n}\right)\right)
$$

é $M R R_{2}$ em $R^{n}$.

1.3.13 Proposição [Proposição, Karlin e Rinott(1980b)] Se $f$ e $g$ são funções $M R R_{2}$. Então, $f g$ é $M R R_{2}$. 


\subsubsection{Observações}

O1 Se $f$ é $M R R_{2}, f \neq 0$. Então, $\frac{1}{f}$ e $M T P_{2}$.

O2 Consideremos as aplicações $f: R^{n} \rightarrow R, M R R_{2}\left(M T P_{2}\right)$ e $g: R \rightarrow R$ tal que $g(x y)=g(x) g(y) \forall x, y \in R$.

(i) Se $g$ é não decrescente. Então, $g \circ f$ é $M R R_{2}\left(M T P_{2}\right)$.

(ii) Se $g$ é não crescente. Então, $g \circ f$ é $M T P_{2}\left(M R R_{2}\right)$.

Caracterizando a classe das funções $S-M R R_{2}$

1.3.15 Proposição [Proposição 2.6., Karlin e Rinott(1980b)] Seja $\underline{X}=\left(X_{1}, \cdots\right.$ ,$\left.X_{n}\right)=\sum_{j=1}^{m} \underline{Z}^{(j)}$ onde $\underline{Z}^{(j)}=\left(Z_{1}^{(j)}, \cdots, Z_{n}^{(j)}\right), j=1, \cdots, m$, são vetores independentes; cada um deles com distribuição $S-M R R_{2}$ em relação à medida de Lebesgue. ou à medida de contagem sobre os inteiros. Suponhamos também que as marginais dos $Z_{i}^{(j)}, i=1, \cdots, n, j=1, \cdots, m$, são $P F_{2}$. Então, para todo conjunto de funções concordantes e $P F_{2},\left\{\varphi_{i}\right\}_{i=1}^{n}$,

$$
E\left\{\prod_{i=1}^{n} \varphi_{i}\left(X_{i}\right)\right\} \leq \prod_{i=1}^{n} E\left\{\varphi_{i}\left(X_{i}\right)\right\}
$$


1.3.16 Proposição [Proposição 3.1., Karlin e Rinott(1980b)] Sejam $Y_{1}, \cdots, Y_{n} S$ $M R R_{2}$ e sejam $\left\{X_{i}\right\}_{i=1}^{n}$, onde $X_{i}=b_{i}\left(X_{i}\right), i=1, \cdots, n$, com funções $b_{i}$ todas crescentes (decrescentes) e côncavas (convexas). Então, para toda seqüência $\left\{\varphi_{\nu}\right\}$ de funções crescentes (decrescentes) e $P F_{2}$, a função $h$ é $M R R_{2}$. Onde

$$
h\left(x_{\nu_{1}}, \cdots, x_{\nu_{k}}\right):=\int_{-\infty}^{+\infty} \cdots \int_{-\infty}^{+\infty} f_{\underline{X}}\left(x_{1}, \cdots, x_{n}\right) \prod_{s=1}^{n-k} \varphi_{s}\left(x_{j_{s}}\right) d x_{j_{1}} \cdots d x_{j_{n-k}},
$$

$\left\{\nu_{1}, \cdots, \nu_{k}\right\}$ e $\left\{j_{1}, \cdots, j_{n-k}\right\}$ são conjuntos complementares, no total de índices $\{1, \cdots, n\}$.

Resultado: Uma função $\psi$ não negativa e diferenciável é $P F_{2}$ se, e somente se, $\psi \psi^{\prime \prime}-\left(\psi^{\prime}\right)^{2} \geq 0$

O resultado anterior aplicado a cada $\psi_{i}=\varphi_{i} \circ b_{j_{i}}$ implica 1.3 .16 , onde $\varphi_{i}$ e $b_{j_{i}}$ são como na hipóteses de 1.3.15 e 1.3.16, respectivamente.

\subsubsection{Perda da "mágica " de 1.3.1 [Karlin e Rinott(1980 a)]:}

A condição $M R R_{2}$ nem sempre é preservada pela marginalização.

Seja $f\left(x_{1}, x_{2}, x_{3}\right)=\exp \left[-x_{3}\left(x_{1}+x_{2}\right)\right]$, então $f$ é $M R R_{2}$ no domínio $x_{i}>0, i=$ 1,2,3. Mas a marginal

$$
\int_{0}^{\infty} f\left(x_{1}, x_{2}, x_{3}\right) d x_{3}=\frac{1}{\left(x_{1}+x_{2}\right)}
$$

não é $R R_{2}$ [Karlin e $\left.\operatorname{Rinott}(1980 \mathrm{~b})\right]$. 
1.3.18 Propriedades [Proschan (1983)]

(P1)Toda subcoleção de duas ou mais variáveis aleatórias $N A$ resulta $N A$.

(P2)Variáveis aleatórias independentes são $N A$.

(P3)Funções crescentes definidas sobre subcoleções disjuntas de um coleção de variáveis $N A$, são $N A$.

(P4)Junção de vetores independentes de variáveis aleatórias $N A$ é $N A$. Isto é: dados $\underline{X}=\left(X_{1}, \cdots, X_{n}\right) N A$ e $\underline{Y}=\left(Y_{1}, \cdots, Y_{m}\right) N A, \underline{X}$ e $\underline{Y}$ vetores aleatórios independentes. Então, o vetor aleatório $(\underline{X}, \underline{Y})$ é $N A$. 


\subsection{Exemplos}

O nosso objetivo procurado na apresentação dos seguintes exemplos é o de conhecer as técnicas usadas para determinar negatividade ou positividade, em relação aos conceitos apresentados. Mais ainda, pode-se dizer que em outros exemplos encontrados na literatura, as técnicas empregadas para testar esses tipos de dependências são as mesmas.

\section{Positividade}

\subsubsection{Exemplo}

Normal Multivariada

Seja $\underline{T}=\left(T_{1}, \cdots, T_{n}\right)$ com distribuição $N_{n}\left(\underline{\mu}, \Sigma_{1}\right)$,

$$
\underline{\mu}=\left(\mu_{1}, \cdots, \mu_{n}\right), \Sigma_{1}^{-1}=S=\left(r_{i j}\right)_{i, j=1}^{n},-\infty<t_{i}<+\infty, i=1, \cdots, n .
$$

Portanto, a densidade de $\underline{T}$ é

$$
f_{\underline{T}}\left(t_{1}, \cdots, t_{n}\right)=\frac{1}{(2 \pi)^{\frac{n}{2}}\left|\Sigma_{1}\right|^{\frac{1}{2}}} \exp \left\{-\frac{1}{2} \sum_{i, j=1}^{n} r_{i j}\left(t_{i}-\mu_{i}\right)\left(t_{j}-\mu_{j}\right)\right\} .
$$

\section{Afirmação}

$$
f_{\underline{T}} \text { é } T P_{2} \text { em pares } \Leftrightarrow r_{i j}<0, i \neq j \Rightarrow \underline{I} \text { é } P A \text { e } f_{\underline{T}} \text { é } M T P_{2} \text {. }
$$


Primeiramente verifiquemos que,

$$
f_{\underline{T}} \text { é } T P_{2} \text { em pares } \Leftrightarrow r_{i j}<0, \forall i \neq j \text {. }
$$

Prova

$f_{\underline{T}}(\underline{t})=C_{1}(\underline{t}) C_{2}(\underline{t}) e^{-r_{i j} t_{i} t_{j}}$. Se fixarmos $t_{s}, s \neq i, s \neq j(i \neq j)$, podemos obter, $C_{1}$ e $C_{2}$ independentes de $t_{i}$ e $t_{j}$ respectivamente. Como $e^{-r_{i j} t_{i} t_{j}}$ é $T P_{2}$ nas variáveis $T_{i}, T_{j}$ se, e somente se, $r_{i j}<0$. [Barlow e Proschan(1981)]. Logo, temos provada a equivalência.

Por 1.3.2 e 1.3.3,

decorre que o vetor $\underline{T}$ é $P A$ e possui f.d.p. $M T P_{2}$, respectivamente.

\section{Negatividade}

\subsubsection{Exemplo}

Distribuição Multinomial .M(m, $\left.p_{1}, \cdots, p_{n}\right)$

$$
\begin{aligned}
& f\left(k_{1}, \cdots, k_{n}\right) \\
& =\left\{\begin{array}{lll}
\left(\begin{array}{c}
m \\
k_{1}, \ldots, k_{n}
\end{array}\right) p_{1}^{k_{1}} \cdots p_{n}^{k_{n}} \quad \text { se } \sum_{i=1}^{n} k_{i}=m & 0 \leq k_{i} . \text { inteiro } \\
0 & & i=1, \ldots, n .
\end{array}\right.
\end{aligned}
$$

A função de densidade de probabilidade da Multinomial é uma função $S-M R R_{2}$ [Karlin e Rinott(1980 b)]. 
Prova: a prova da afirmação prévia decorre das seguintes três Proposições e do Teorema, apresentados seguidamente.

Proposição : Para $n=2, f\left(k_{1}, k_{2}\right)$ é $R R_{2}$.

Dada $f$ como no exemplo $\left(M R R_{2}\right)$; veremos, como funções $P F_{2}$ dão lugar a uma função $R R_{2}$ em pares e depois como seqüências de funções $P F_{2}$ dão lugar a uma função $M R R_{2}$, resultando $f, S-M R R_{2}$.

Proposição : Seja $a_{k} . k \geq 0$, uma seqüência de funções $P F_{2}$ nos subíndices $k$. Então,

$$
g\left(k_{2}, \cdots, k_{n}\right):=\sum_{\nu=0}^{m} a_{\nu} f\left(\nu, k_{2}, \cdots, k_{n}\right)
$$

é $R R_{2}$ em cada par $k_{i}, k_{j} .2 \leq i<j \leq n$, quando as outras variáveis ficam fixas.

A Proposição é provada notando que para toda $(n-1)$-upla fixa, $\left(k_{2}, \cdots, k_{n}\right)$,

$$
g\left(k_{2}, \cdots, k_{n}\right)=a_{k_{1}} \frac{m !}{k_{1} ! \cdots k_{n} !} \prod_{i=1}^{n} p_{i}^{k_{i}}, \quad k_{1}=m-k_{2}-\cdots-k_{n}
$$

e tendo presente que a condição $R R_{2}$ é preservada pelo produto.

Proposição : Seja $d_{k} . k \geq 0$, uma seqüência de funções $P F_{2}$. Então,

$$
g^{*}\left(k_{3}, \cdots, k_{n}\right):=\sum_{\nu=0}^{m} d_{\nu} g\left(\nu, k_{3}, \cdots, k_{n}\right)
$$

é $R R_{2}$ em cada par $k_{i}, k_{j}, 3 \leq i<j \leq n$, quando as outras variáveis ficam fixas; com $g$ como na Proposição anterior.

Isso é provado usando a fórmula da composição [Karlin e Rinott(1963)], citada na 
Seção 2.

Teorema: Sejam $\left\{a_{k}^{(1)}\right\}, \cdots,\left\{a_{k}^{(n-2)}\right\}, k \geq 0$, seqüências $P F_{2}$. Seja, $g\left(k_{\mu+1}, \cdots, k_{n}\right):=\sum_{k_{\mu}} \cdots \sum_{k_{1}} \prod_{j=1}^{\mu} a_{k_{j}}^{(j)} f\left(k_{1}, \cdots, k_{n}\right) \quad 1 \leq \mu \leq n-2$.

Onde $f$ é a multinomial. Então, $g\left(k_{\mu+1}, \cdots, k_{n}\right)$ é $M R R_{2}$ e assim $f\left(k_{1}, \cdots, k_{n}\right)$ resulta $S-M R R_{2}$.

As primeiras duas Proposições são usadas para o caso $\mu=1$. No entanto, os passos seguidos na prova da Proposição anterior completam esta prova.

\section{Afirmação}

O vetor aleatório $\left(K_{1}, \cdots, K_{n}\right)$ com distribuição de probabilidade dada pela Multinomial é um vetor $N A$ [Proschan(1983)].

Prova da Afirmação:

Considere $\underline{K}=\left(K_{1}, \cdots, K_{n}\right)$ vetor aleatório de um único experimento com distribuição multinomial (i.e. existe um único $i$ tal que $K_{i}=1$ ). Logo,

$$
f_{\underline{K}}\left(k_{1}, \cdots, k_{n}\right)=\left\{\begin{array}{ccc}
\frac{p_{1}^{k_{1} \cdots p_{n} k_{n}}}{k_{1} ! \cdots k_{n} !} & \text { se } \sum_{i=1}^{n} k_{i}=1, \sum_{i=1}^{n} p_{i}=1 & 0 \leq k_{i} \in Z \\
0 & \text { caso contrário } & i=1, \ldots, n .
\end{array}\right.
$$

Sejam $A_{1}$ e $A_{2}$ partições disjuntas de $\{1, \cdots, n\}, f_{1}$ e $f_{2}$ duas funções crescentes atuando sobre cada conjunto de variáveis aleatórias induzido pelas partições anteriores.

$$
\operatorname{Cov}\left\{f_{1}\left(k_{i}, i \in A_{1}\right), f_{2}\left(k_{j}, j \in A_{2}\right)\right\}=L_{1}+L_{2}
$$




$$
\begin{aligned}
& L_{1}:=\operatorname{Cov}\left\{E\left(f_{1}\left(k_{i}, i \in A_{1}\right) \mid I\right), E\left(f_{2}\left(k_{j}, j \in A_{2}\right) \mid I\right)\right\} \\
& L_{2}:=E\left\{\operatorname{Cov}\left(f_{1}\left(k_{i}, i \in A_{1}\right), f_{2}\left(k_{j}, j \in A_{2}\right) \mid I\right)\right\},
\end{aligned}
$$

onde $I$ é a variável aleatória indicadora da posição do 1 .

Para o caso $n=2$, é possível usar a equivalência: $N A$ se, e somente se, $N O D$ (cuja prova apresentaremos mais em frente).

Assumamos que, para $n$-1, $\operatorname{Cov}\left\{f_{1}\left(k_{i}, i \in A_{1}\right), f_{2}\left(k_{j}, j \in A_{2}\right)\right\} \leq 0$; logo, a partir do conhecimento da variável $I, L_{2} \leq 0$, por hipóteses indutiva. Vamos supor que o valor 1 é admitido em $\left\{k_{i}, i \in A_{1}\right\}$; então, $E\left(f_{1}\left(k_{i}, i \in A_{1}\right) \mid I\right)$ pode tomar, no máximo, $\left|A_{1}\right|<n$ valores diferentes. No entanto, $E\left(f_{2}\left(k_{j}, j \in A_{2}\right) \mid I\right)=E\left(f_{2}(\underline{0})\right)$ e como $f_{1}$ é crescente, $E\left(f_{1}\left(k_{i}, i \in A_{1}\right) \mid I\right)$ e $E\left(f_{2}\left(k_{j}, j \in A_{2}\right) \mid I\right)$ tomarão valores discordantes, e aplicando hipóteses indutiva, $L_{1} \leq 0$.

Agora, a Multinomial de parâmetros $\left(m, p_{1}, \cdots, p_{n}\right), m>1$ poderia ser vista como a convolução de $m$ diferentes e independentes Multinomiais de parâmetro $\left(1, p_{1} \cdots, p_{n}\right)$. Então, a partir de 1.3.18, (P4) [Proschan(1983)] citada previamente,

$$
M\left(m, p_{1}, \cdots, p_{n}\right) \text { é } N A \text {. }
$$

1.4.3 Exemplo - Processo de Poisson: Um número fixo de bactérias são colocadas numa placa contendo nutrientes que permitem a multiplicação das bactérias. Após alguns dias, onde tínhamos cada bactéria há uma colônia delas. A localização de cada colônia e o número total de bactérias na placa é agora aleatório. A localização das colônias pode ser vista como um subconjunto aleatório de pontos na placa. 
Chamemos de $K$ o número total de partículas na placa $S$ e para cada $B$ em $S, K_{B}$ denota o número de partículas presentes em $B$. Consistente com isso, $K=K_{S}$.

Seja $m$ o número fixo, original de partículas, com localização aleatória em $S$. Se as $m$ partículas são independentes e uniformemente distribuídas sobre um conjunto $S$ de volume finito $|S|$, então, cada partícula tem probabilidade $p=\frac{|B|}{|S|}$ de ficar em $B$; conseqüentemente, o número de partículas $K_{B}$ que estão em $B$ tem distribuição Binomial de parâmetros $m$ e $p$.

Formalizando, se $\left\{B_{i}\right\}_{i=1}^{n}$ são subconjuntos disjuntos de $S, S=\bigcup_{i=1}^{n} B_{i}$ e $p_{i}=$ $\frac{\left|B_{i}\right|}{|S|}$, logo $\left(K_{B_{1}}, \cdots, K_{B_{n}}\right)$ tem distribuição $M\left(m, p_{1}, \cdots, p_{n}\right)$ e quando $\sum_{i=1}^{n} p_{i}=$ $1, \sum_{i=1}^{n} k_{B_{i}}=m$

$$
P\left(K_{B_{1}}=k_{B_{1}}, \cdots, K_{B_{n}}=k_{B_{n}}\right)=\frac{m ! p_{1}^{k_{B_{1}}} \cdots p_{n}^{k_{B_{n}}}}{k_{B_{1}} ! \cdots k_{B_{n}} !}=\frac{m !}{|S|^{m}} \prod_{i=1}^{n} \frac{\left|B_{i}\right|^{k_{B_{i}}}}{k_{B_{i}} !}
$$

Vamos supor, agora, que $K$ é uma variável aleatória com distribuição de Poisson de parâmetro $\lambda|S|$ e suponhamos que dado $K=m$, as partículas são independentes e uniformemente distribuídas sobre a placa $S$. Assim,

$$
\begin{aligned}
P\left(K_{B_{1}}=k_{B_{1}}, \cdots, K_{B_{n}}=k_{B_{n}} \mid K=m\right) & =\frac{m !}{|S|^{m}} \prod_{i=1}^{n} \frac{\left|B_{i}\right|^{k_{B_{2}}}}{k_{B_{1}} !} \text {, indep. } \\
P\left(K_{B_{1}}=k_{B_{1}}, \cdots, K_{B_{n}}=k_{B_{n}}, K=m\right) & =P(K=m) \frac{m !}{|S|^{m}} \prod_{i=1}^{n} \frac{\left|B_{i}\right|^{k_{B_{i}}}}{k_{B_{i}} !} \\
& =\frac{\lambda^{m}|S|^{m}}{m !} e^{-\lambda|S|} \frac{m !}{|S|^{m}} \prod_{i=1}^{n} \frac{\left|B_{i}\right|^{k_{B_{1}}}}{k_{B_{1}} !} \\
& =\lambda^{m} e^{-\lambda|S|} \prod_{i=1}^{n} \frac{\left|B_{i}\right|^{k_{B_{i}}}}{k_{B_{i}} !},
\end{aligned}
$$

e como $|S|=\sum_{i=1}^{n}\left|B_{i}\right|$ e $m=\sum_{i=1}^{n} k_{B_{i}}$,

$$
P\left(K_{B_{1}}=k_{B_{1}}, \cdots, K_{B_{n}}=k_{B_{n}}\right)=\prod_{i=1}^{n} \frac{\left[\lambda\left|B_{i}\right|\right]^{k_{B_{i}}}}{k_{B_{i}} !} e^{-\lambda\left|B_{i}\right|},
$$




$$
\text { se } \sum_{i=1}^{n} p_{i}=1 \text { e } \sum_{i=1}^{n} k_{B_{i}}=m \text {. }
$$

A partir desse panorama, $K_{B_{1}}, \cdots, K_{B_{n}}$ são independentes com distribuição Poisson de Parâmetros $\lambda\left|B_{i}\right|$ respectivamente. E ainda, consideremos cada Poisson de parâmetro $\lambda\left|B_{i}\right|$ como função de $k_{B_{i}}$ e $\left|B_{i}\right|$, assim, cada uma delas, é $T P_{2}$. Logo, as densidades Poisson $\left(\lambda\left|B_{i}\right|\right)$ são densidades $P F_{2}$ [ Barlow e Proschan(1981) pp.92]. Então, pelo Teorema 2.8 [Proschan(1983)]

$$
K_{B_{1}}, \cdots, K_{B_{n}} \mid \sum_{i=1}^{n} k_{B_{i}}=m \text { são } N A
$$

\subsubsection{Exemplo}

Normal Multivariada

Seja $\underline{Y}=\left(Y_{1}, \cdots, Y_{t}\right)$ com distribuição $N_{t}\left(\underline{0}, \Sigma_{2}\right)$, onde $\Sigma_{2}=\Lambda-\left\|\alpha_{i} \alpha_{j}\right\|_{i, j=1}^{t}$ e

$$
\Lambda=\operatorname{diag}\left(\lambda_{1}, \cdots, \lambda_{t}\right), \lambda_{i}>0, \alpha_{i} \geq 0, i=1, \cdots, t
$$

Logo,

$$
\Sigma_{2}^{-1}=\Lambda^{-1}+\gamma\left\|\beta_{i} \beta_{j}\right\|_{i, j=1}^{t} \operatorname{com} \gamma=\frac{1}{1-\sum_{i=1}^{t} \lambda_{i}^{-1} \alpha_{i}^{2}}
$$

Se

$$
\gamma>0 \text { ou equivalentemente } \sum_{i=1}^{t} \lambda_{i}^{-1} \alpha_{i}^{2}<1 \text {, a distribuição é } S-M R R_{2} \text {. }
$$

Roteiro da prova: Primeiramente, o resultado é provado para o caso $t=2$, usando para isso a fórmula da composição 1.2.21. Em seguida, os passos propostos para a prova da Multinomial como densidade $S-M R R_{2}$ [Karlin e Rinott (1980b); Fahmy, Pereira et al.(1982)]. 
A seguir, apresentamos outros exemplos de aplicações $R R_{2}$

\subsubsection{Formas Quadráticas Homogêneas :}

\section{Exemplo A}

$$
Q_{1}: R^{2} \rightarrow R, Q_{1}(x, y):=x^{2}+y^{2}
$$

Parabolóide Positivo Definido $\left(Q_{1}(x, y)>0\right.$ exceto quando $\left.(x, y)=(0,0)\right)$. Resulta $R R_{2}$ quando $x \in[0,+\infty), y \in[0,+\infty)$ ou (excludente), a forma é $R R_{2}$ se $x \in$ $(-\infty, 0], y \in(-\infty, 0]$.

\section{Exemplo B}

$$
Q_{2}: R^{2} \rightarrow R, Q_{2}(x, y):=y^{2}-x^{2}
$$

Polinômio não Positivo Definido. Resulta $R R_{2}$ se $x \in[0,+\infty), y \in(-\infty, 0]$ ou (excludente), a forma é $R R_{2}$ se $x \in(-\infty, 0], y \in[0,+\infty)$.

\section{Exemplo C}

Se,

$$
Q(x, y):=(x, y)\left(\begin{array}{ll}
a & b \\
c & d
\end{array}\right)\left(\begin{array}{l}
x \\
y
\end{array}\right)=a x^{2}+(b+c) x y+d y^{2}
$$

satisfaz a condição $C_{i}$, então $Q$ é $R R_{2}$ em $D_{i}$, domínio em $R^{2}$. 
$\mathrm{C} 1: \operatorname{sinal}(a d)=\operatorname{sinal}(a(b+c))=\operatorname{sinal}(d(b+c))=+. D_{1}=[0, \infty) \times[0, \infty)$.

C2: $\operatorname{sinal}(a d)=+, \operatorname{sinal}(a(b+c))=\operatorname{sinal}(d(b+c))=-. D_{2}=(-\infty, 0] \times(-\infty, 0]$.

C3: $\operatorname{sinal}(a d)=+; b+c=0 . D_{3}=[0, \infty) \times[0, \infty)$.

C4: $\operatorname{sinal}(a d)=+; b+c=0 . \quad D_{4}=(-\infty, 0] \times(-\infty, 0]$.

C5: $\operatorname{sinal}(a d)=-; b+c=0 . D_{5}=[0, \infty) \times(-\infty, 0]$.

C6: $\operatorname{sinal}(a d)=-; b+c=0 . D_{6}=(-\infty, 0] \times[0, \infty)$.

A verificação disto decorre da seguinte afirmação.

Se.

$a d\left(y^{2}-y^{\prime 2}\right)\left(x^{\prime 2}-x^{2}\right) \geq 0, \quad a(b+c)\left(y-y^{\prime}\right) x^{\prime} x\left(x^{\prime}-x\right) \geq 0, \quad d(b+c)\left(x^{\prime}-x\right) y y^{\prime}\left(y-y^{\prime}\right) \geq 0$, então, $Q$ é $R R_{2}$ num domínio de $R^{2}$.

Assim. os Exemplos $\mathrm{A}$ e $\mathrm{B}$ são conseqüência das condiçōes $C_{3}, C_{4}$ e $C_{5}, C_{6}$, respectivamente. 


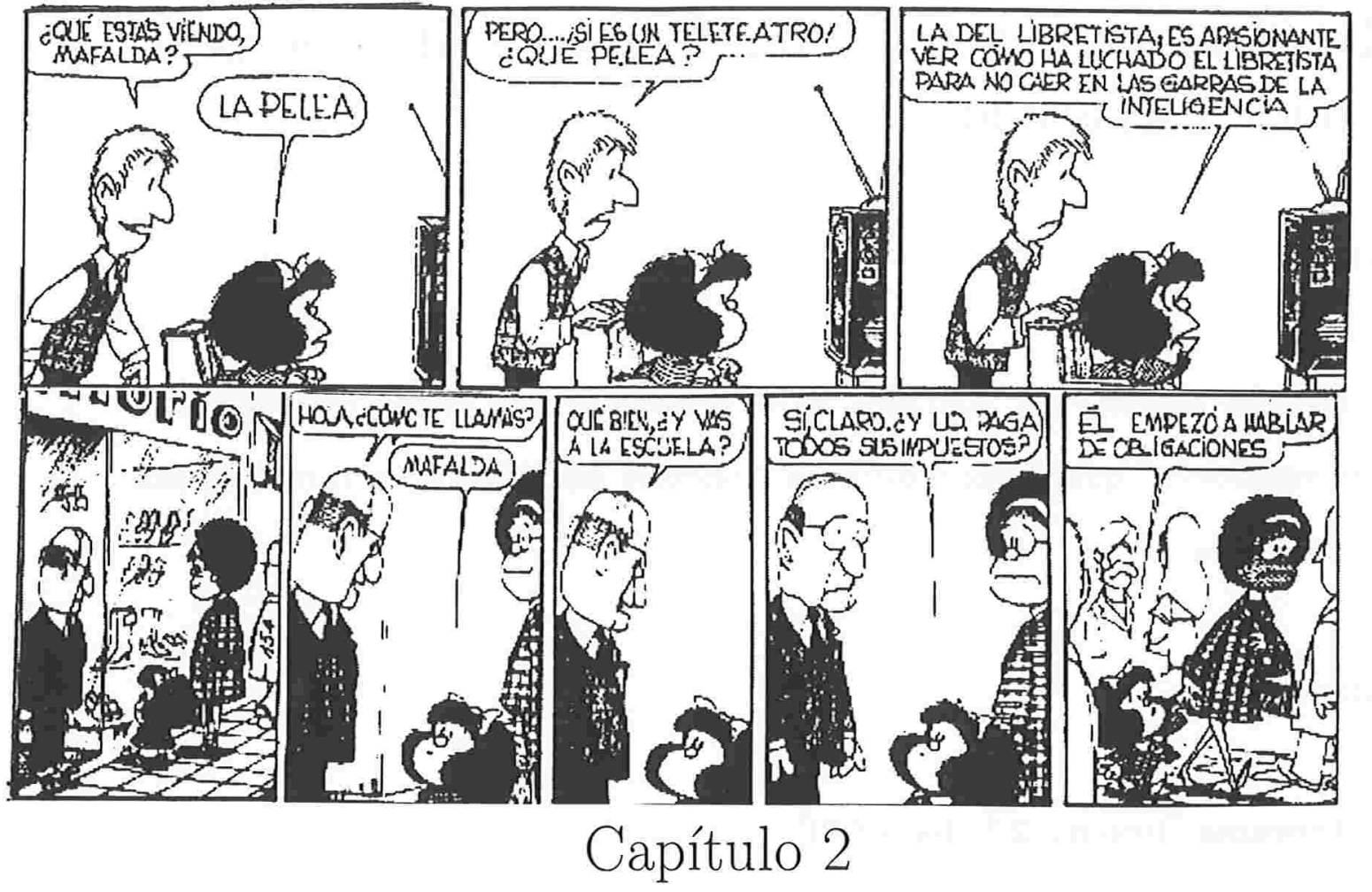

\section{Propriedades das Dependências Positivas e Negativas}

- Seção 2.1: Conexões entre alguns dos Conceitos de Positividade (Negatividade)

- Seção 2.2: Critérios para Examinar um Vetor Aleatório com Respeito à Estrutura de Associação entre suas Variáveis

- Seção 2.3: Mistura de Associações: Vetores Aletórios contendo os dois Tipos de Associações

- Seção 2.4: Complementos das Seções 2 e 3

- Seção 2.5: Herança da condição PA 
2.1. Conexões entre alguns dos conceitos de positividade (negatividade).

\section{Objetivo}

Conhecidas algumas das "conexões" entre os diversos tipos de conceitos de positividade, estabelecer, quando for possível as "conexões" equivalentes entre os conceitos de negatividade.

\section{Positividade}

2.1.1 Teorema [Teorema 2.3, Joe(1997)]

(a) f.d.p.TP $P_{2} \Rightarrow S I \Rightarrow L T D, R T I$;

(b) $L T D$ ou $R T I \Rightarrow P A \Rightarrow P Q D$;

(c) f.d.p. $T P_{2} \Rightarrow$ f.d.a. $T P_{2}$ e função de sobrevivência $T P_{2}$;

(d) f.d.a. $T P_{2} \Rightarrow L T D$, e função de sobreviência $T P_{2} \Rightarrow R T I$.

É possível assegurar as mesmas relações, no caso da dependência negativa?. 


\section{Negatividade}

A noção de $S D$ representa uma condição de dependência negativa, pois $X_{2}$ é menos provável à medida que $X_{1}$ cresce, assumindo valores "grandes".

No entanto, os conceitos de $N D S$ e $C D S$ tentam generalizar o conceito de $S D$.

$R T D$ e $L T I$, podem ser enquadrados dentro da dependência negativa. No primeiro conceito, $X_{2}$ é menos provável à medida que $X_{1}$ atinge valores grandes, ou seja $X_{2}$ não poderá atingir valores grandes. O segundo conceito apresenta $X_{2}$ como mais provável à medida que $X_{1}$ cresce, mas $X_{2}$ só pode atingir um certo valor $x_{2}$.

No seguinte Teorema procuramos estabelecer condições equivalentes às presentes no Teorema anterior, dentro do contexto de negatividade.

\subsubsection{Teorema*}

(a) f.d.p. $R R_{2} \Rightarrow S D$ :

(b) $N A \Leftrightarrow N Q D$ :

(c) f.d.p. $R R_{2} \Rightarrow$ f.d.a. $R R_{2}$ e função de sobrevivência $R R_{2}$;

(d)f.d.a. $R R_{2} \Rightarrow L T I$, e função de sobrevivência $R R_{2} \Rightarrow R T D$. 
Prova de 2.1.2

(a): dados $x<x^{\prime}$, verificar $P\left(X_{2}>y \mid X_{1}=x\right) \geq P\left(X_{2}>y \mid X_{1}=x^{\prime}\right)$ é equivalente a provar que,

$$
\int_{y}^{\infty} f(x, z) d z \int_{-\infty}^{+\infty} f\left(x^{\prime}, w\right) d w \geq \int_{y}^{\infty} f\left(x^{\prime}, z\right) d z \int_{-\infty}^{+\infty} f(x, w) d w
$$

ou

$$
\int_{y}^{\infty} \int_{-\infty}^{+\infty}\left[f(x, z) f\left(x^{\prime}, w\right)-f\left(x^{\prime}, z\right) f(x, w)\right] d w d z \geq 0
$$

onde $f$ é $f . d . p$. conjunta. Agora, sabemos que, se $w \leq z$,

$$
f(x, z) f\left(x^{\prime}, w\right)-f\left(x^{\prime}, z\right) f(x, w) \geq 0,
$$

portanto, é válido que,

$$
f(x, z) f\left(x^{\prime}, w\right)-f\left(x^{\prime}, z\right) f(x, w) \geq 0, \quad \forall(z, w) \in(y, \infty] \times(-\infty, y]
$$

$\log$,

$$
\int_{y}^{\infty} \int_{-\infty}^{y}\left[f(x, z) f\left(x^{\prime}, w\right)-f\left(x^{\prime}, z\right) f(x, w)\right] d w d z \geq 0 .
$$

Notemos que

$$
\int_{y}^{\infty} \int_{y}^{+\infty}\left[f(x, z) f\left(x^{\prime}, w\right)-f\left(x^{\prime}, z\right) f(x, w)\right] d w d z \geq 0
$$

(b): $\Rightarrow$ direta, desde as definições respectivas.

$\Leftarrow$ : provemos a afirmação para funções indicadoras: sejam $A$ e $B$ conjuntos borelianos da reta. Sempre que as funções $I_{A}(x)$ e $I_{B}(y)$ sejam concordantes, como $X$ e $Y$ são $N Q D$, temos

$$
E\left\{I_{A}(X) I_{B}(Y)\right\} \leq E\left\{I_{A}(X)\right\} E\left\{I_{B}(Y)\right\}
$$


como conseqüência direta, dadas duas coleções de valores não negativos $\left\{x_{i}\right\}_{i=1}^{m},\left\{y_{j}\right\}_{j=1}^{k}$ e borelianos da reta $\left\{A_{i}\right\}_{i=1}^{m},\left\{B_{j}\right\}_{j=1}^{k}$, respectivamente,

$$
E\left\{\sum_{i=1}^{m} x_{i} I_{A_{i}}(X) \sum_{j=1}^{k} y_{j} I_{B_{j}}(Y)\right\} \leq E\left\{\sum_{i=1}^{m} x_{i} I_{A_{i}}(X)\right\} E\left\{\sum_{j=1}^{k} y_{j} I_{B_{j}}(Y)\right\} .
$$

Ou seja, a afirmação é válida para o espaço das funções simples.

Consideremos agora duas funções Borel-mensuráveis, monótonas crescentes, não negativas, $f$ e $g$. Então. existem duas seqüências de funções simples $\left\{f_{n}\right\}_{n=1}^{+\infty}$ e $\left\{g_{n}\right\}_{n=1}^{+\infty}$, não negativas, que convergem crescentemente para $f$ e $g$ respectivamente [Teorema 1.5.5. Ash(1972) pp.38]. Logo, pelo Teorema da Convergência Monótona aplicado a $f_{n}, f ; g_{n}, g ; f_{n} g_{n} ; f g$, respectivamente, torna válida a afirmação.

Consideremos, por último, duas funções Borel-mensuráveis. monótonas crescentes, $f$ e $g$. Como $f=f^{+}-f^{-}$e $g=g^{+}-g^{-}$onde $f^{+}, f^{-}, g^{+}, g^{-}$são funções Borelmensuráveis, monótonas, não negativas, notar que

$$
\begin{aligned}
& E\left\{f^{+}(X) g^{+}(Y)\right\} \leq E\left\{f^{+}(X)\right\} E\left\{g^{+}(Y)\right\}, \\
& E\left\{f^{-}(X) g^{-}(Y)\right\} \leq E\left\{f^{-}(X)\right\} E\left\{g^{-}(Y)\right\},
\end{aligned}
$$

pois as funções envolvidas são concordantes, $f^{+}=f \vee 0, g^{+}=g \vee 0$,

$$
\begin{aligned}
& E\left\{f^{+}(X) g^{-}(Y)\right\} \geq E\left\{f^{+}(X)\right\} E\left\{g^{-}(Y)\right\}, \\
& E\left\{f^{-}(X) g^{+}(Y)\right\} \geq E\left\{f^{-}(X)\right\} E\left\{g^{+}(Y)\right\},
\end{aligned}
$$

pois as funções envolvidas são discordantes, $f^{-}=(-f) \vee 0, g^{-}=(-g) \vee 0$. A partir disso se conclui,

$$
E\{f(X) g(Y)\} \leq E\{f(X)\} E\{g(Y)\} .
$$


Notemos que em geral, $N U O D$ e/ou $N L O D$ não implica em $N A$ [Proschan(1983) pp.289].

(c): Consideremos previamente a seguinte equivalência

$x_{1}<y_{1}, x_{2}<y_{2} ; F$, f.d.a.é $R R_{2}$ se. e somente se,

$$
F\left(x_{1}, x_{2}\right) F\left(y_{1}, y_{2}\right)-F\left(x_{1}, y_{2}\right) F\left(y_{1}, x_{2}\right) \leq 0
$$

se. e somente se,

$$
\begin{aligned}
F\left(x_{1}, x_{2}\right)\left[F\left(y_{1}, y_{2}\right)-F\left(y_{1}, x_{2}\right)-F\left(x_{1}, y_{2}\right)+F\left(x_{1}, x_{2}\right)\right] \\
-\left[F\left(x_{1}, y_{2}\right)-F\left(x_{1}, x_{2}\right)\right]\left[F\left(y_{1}, x_{2}\right)-F\left(x_{1}, x_{2}\right)\right] \leq 0 .
\end{aligned}
$$

Agora, por hipótese, temos que,

$$
f\left(s_{1}, s_{2}\right) f\left(t_{1}, t_{2}\right)-f\left(s_{1}, t_{2}\right) f\left(t_{1}, s_{2}\right) \leq 0, \quad s_{2}<t_{2}, s_{1}<t_{1} ; f, f . d . p .
$$

Dados $x_{1}<y_{1}, x_{2}<y_{2}$

e considerando, $-\infty \leq s_{2}<x_{2}<t_{2} \leq y_{2}$ e $-\infty \leq s_{1}<x_{1}<t_{1} \leq y_{1}$, verifica-se

$$
\int_{-\infty}^{x_{1}} \int_{-\infty}^{x_{2}} \int_{x_{1}}^{y_{1}} \int_{x_{2}}^{y_{2}}\left[f\left(s_{1}, s_{2}\right) f\left(t_{1}, t_{2}\right)-f\left(s_{1}, t_{2}\right) f\left(t_{1}, s_{2}\right)\right] d t_{2} d t_{1} d s_{2} d s_{1} \leq 0,
$$

equivalentemente,

$$
\begin{aligned}
F\left(x_{1}, x_{2}\right)\left[F\left(y_{1}, y_{2}\right)\right. & \left.-F\left(y_{1}, x_{2}\right)-F\left(x_{1}, y_{2}\right)+F\left(x_{1}, x_{2}\right)\right] \\
\leq & {\left[F\left(x_{1}, y_{2}\right)-F\left(x_{1}, x_{2}\right)\right]\left[F\left(y_{1}, x_{2}\right)-F\left(x_{1}, x_{2}\right)\right], }
\end{aligned}
$$


finalmente, $F$ é $R R_{2}$.

(d):f.d.a. $R R_{2} \Rightarrow L T I$ :

$$
F \text { é } R R_{2} \text {, se. e somente se, } \frac{F\left(x_{1}, x_{2}\right)}{F\left(x_{1}, y_{2}\right)} \leq \frac{F\left(y_{1}, x_{2}\right)}{F\left(y_{1}, y_{2}\right)}, x_{1}<y_{1}, x_{2}<y_{2} \text {, }
$$

se consideramos $y_{2} \rightarrow+\infty$ a desigualdade anterior fica

$$
\frac{F\left(x_{1}, x_{2}\right)}{F_{1}\left(x_{1}\right)} \leq \frac{F\left(y_{1}, x_{2}\right)}{F_{1}\left(y_{1}\right)}
$$

$\operatorname{logo}, \frac{F\left(\cdot, x_{2}\right)}{F_{1}(\cdot)}$ é crescente.

\section{Positividade}

\subsubsection{Teorema [Teorema 2.4, Joe(1997)pp.27]}

(a) Todo subvetor de um vetor aleatório $P A$ é $P A$;

(b) $P A \Rightarrow P U O D$ e $P L O D$;

(c) $P D S \Rightarrow P U O D$ e $P L O D$ :

(d) $C I S \Rightarrow P A$. 


\section{Negatividade}

\subsubsection{Teorema*}

(a) Todo subvetor de um vetor aleatório $N A$ é $N A$ [Joag-Dev e Proschan(1983)pp.288;

$\operatorname{Kim}(1995)$ pp.852];

(b) $N . A \Rightarrow$ NUOD e NLOD [Joag-Dev e Proschan (1983)pp.288];

(c) $N D S \Rightarrow N U O D$ e $N L O D$;

(d) NA não implica em CDS [Joag-Dev e Proschan (1983)pp.291];

(e) f.d.p $S-M R R_{2} \Rightarrow N U O D$ e $N L O D$ [Karlin e Rinott(1980 b)pp.505].

Prova de 2.1.4:

(c).VDS $\Rightarrow$ NUOD:

Dados $x_{j}, x_{j}^{\prime}$, tal que $x_{j}>x_{j}^{\prime}$, por hipótese temos que

$$
P\left(X_{i}>x_{i}, i \neq j \mid X_{j}=x_{j}\right) \leq P\left(X_{i}>x_{i}, i \neq j \mid X_{j}=x_{j}^{\prime}\right)
$$

conseqüentemente,

$$
\frac{\int_{x,}^{\infty} P\left(X_{i}>x_{i}, i \neq j \mid X_{j}=x\right) d F_{j}(x)}{\int_{x_{j}}^{\infty} d F_{j}(x)} \leq \frac{\int_{x_{j}^{\prime}}^{\infty} P\left(X_{i}>x_{i}, i \neq j \mid X_{j}=x\right) d F_{j}(x)}{\int_{x_{j}^{\prime}}^{\infty} d F_{j}(x)} .
$$


Consideremos $j=1$ e $x_{j}^{\prime} \rightarrow-\infty$, então, a desigualdade anterior fica,

$$
P\left(X_{i}>x_{i}, i=1, \cdots, n\right) \leq P\left(X_{1}>x_{1}\right) P\left(X_{i}>x_{i}, i=2, \cdots, n\right) .
$$

Em particular, $\left(X_{2}, \cdots, X_{n}\right)$ é $N D S$ e podemos aplicar a mesma argumentação, i.e.,

$$
P\left(X_{i}>x_{i}, i=2, \cdots, n\right) \leq P\left(X_{2}>x_{2}\right) P\left(X_{i}>x_{i}, i=3, \cdots, n\right) .
$$

Assim, progressivamente obtemos NUOD.

Um procedimento semelhante prova $N D S \Rightarrow N L O D$.

(e) f.d.p. $S-M R R_{2} \Rightarrow N U O D$ e $N L O D$ : para a prova enunciamos e provamos o seguinte Teorema,

2.1.5 Teorema: Seja $\underline{X}=\left(X_{1}, \cdots, X_{n}\right)$, vetor aleatório tomando valores em $R^{n}$, com densidade de probabilidade $f$, com relação à medida produto $\sigma=\sigma_{1} \times \cdots \times \sigma_{n}$, e suponha $f, S-M R R_{2}$. Sejam $\varphi_{1}, \cdots, \varphi_{n}$, funções concordantes e $P F_{2}$, então, para qualquer $k$ tal que $1 \leq k \leq n$,

$$
E\left[\prod_{i=1}^{n} \varphi_{i}\left(X_{i}\right)\right] \leq E\left[\prod_{i=1}^{k} \varphi_{i}\left(X_{i}\right)\right] E\left[\prod_{i=k+1}^{n} \varphi_{i}\left(X_{i}\right)\right],
$$

sempre que as esperanças existam.

Prova de 2.1.5:

Considerando o seguinte Lema, desenvolvemos a prova para o caso $n=2\left(f\left(x_{1}, x_{2}\right) R R_{2}\right)$; conseqüentemente, procedemos por indução em $n$.

Notação : $d x_{i}=d \sigma_{i}\left(x_{i}\right)$. 
2.1.6 Lema: Se $f\left(x_{1}, x_{2}\right)$ é $R R_{2}$ e $h_{1}, h_{2}$ são crescentes (ou decrescentes) e absolutamente integráveis com respeito a $f$, então

$$
\iint h_{1}\left(x_{1}\right) h_{2}\left(x_{2}\right) f\left(x_{1}, x_{2}\right) d x_{1} d x_{2} \leq \int h_{1}\left(x_{1}\right) f_{1}\left(x_{1}\right) d x_{1} \int h_{2}\left(x_{2}\right) f_{2}\left(x_{2}\right) d x_{2}
$$

onde $f_{i}\left(x_{i}\right), i=1,2$, são as densidades marginais da $f$.

Prova de 2.1.6:

Fato 1: Se

$$
g\left(x_{1}, x_{2}\right) \text { é } R R_{2} \quad \text { e } \int g\left(x_{1}, x_{2}\right) d x_{2} \equiv 1,
$$

então para qualquer função $h$, crescente (ou decrescente) a transformada

$$
\int h\left(x_{2}\right) g\left(x_{1}, x_{2}\right) d x_{2}
$$

é decrescente (ou crescente) em $x_{1}$. [Karlin (1968)-pp 22-Proposição 3.1]

Fato 2: Se $t(x)$ é densidade, $s(x)$, transformação crescente, $u(x)$, transformação decrescente em $x \in R$. Então, pela desigualdade de Tchebycheff,

$$
\int s(x) u(x) t(x) d x \leq \int s(x) t(x) d x \int u(x) t(x) d x
$$

Seja.

$$
u\left(x_{1}\right):=\int h_{2}\left(x_{2}\right)\left(f\left(x_{1}, x_{2}\right) / f_{1}\left(x_{1}\right)\right) d x_{2} .
$$

A integral do segundo termo é decrescente (ou crescente) em $x_{1}$; pelo Fato 1.

$u$ é decrescente (ou crescente) em $x_{1}$. 
$h_{1}$ é crescente (ou decrescente) em $x_{1}$ ( faz o papel de $s$ no Fato 2).

$f_{1}$, f.d.p. (faz o papel de $t$ no Fato 2).

Assim, provamos 2.1.6.

Retomamos a prova de 2.1.5: consideremos a densidade

$$
f_{2}^{* n}\left(x_{2}, \cdots, x_{n}\right):=\int \varphi_{1}\left(x_{1}\right) f_{1}^{n}\left(x_{1}, \cdots, x_{n}\right) d x_{1} / \int \varphi_{1}\left(x_{1}\right) f_{1}\left(x_{1}\right) d x_{1},
$$

onde

$$
f_{i}^{j}\left(x_{i}, \cdots, x_{j}\right)=\int \cdots \int f\left(x_{1}, \cdots, x_{n}\right) d x_{1} \cdots d x_{i-1} d x_{j+1} \cdots d x_{n},
$$

para $1 \leq i \leq j \leq n$

Definamos,

$$
E_{f_{1}}\left[\varphi_{1}\left(X_{1}\right)\right]:=\int \varphi_{1}\left(x_{1}\right) f_{1}\left(x_{1}\right) d x_{1}
$$

Aceitemos 2.1.5 válido para o caso $n-1$. Em seguida, provaremos 2.1.5 para o caso $n$, usando a hipótese na densidade definida por $f_{2}^{* n}\left(x_{2}, \cdots, x_{n}\right)$.

Como,

$$
E_{f_{1}^{n}}\left[\prod_{i=1}^{n} \varphi_{i}\left(X_{i}\right)\right]=E_{f_{2}^{* n}}\left[\prod_{i=2}^{n} \varphi_{i}\left(X_{i}\right)\right] E_{f_{1}}\left[\varphi_{1}\left(X_{1}\right)\right],
$$

e, por hipótese indutiva, para $2 \leq k \leq n$,

$$
E_{f_{2}^{* n}}\left[\prod_{i=2}^{n} \varphi_{i}\left(X_{i}\right)\right] \leq E_{f_{2}^{* n}}\left[\prod_{i=2}^{k} \varphi_{i}\left(X_{i}\right)\right] E_{f_{2}^{* n}}\left[\prod_{i=k+1}^{n} \varphi_{i}\left(X_{i}\right)\right] .
$$

Logo,

$$
E_{f_{2}^{* n}}\left[\prod_{i=2}^{k} \varphi_{i}\left(X_{i}\right)\right]=\frac{1}{E_{f_{1}}\left[\varphi_{1}\left(X_{1}\right)\right]} E_{f_{1}^{n}}\left[\prod_{i=1}^{k} \varphi_{i}\left(X_{i}\right)\right]
$$




$$
E_{f_{2}^{* n}}\left[\prod_{i=k+1}^{n} \varphi_{i}\left(X_{i}\right)\right]=E_{f_{1}^{n}}\left[\prod_{i=k+1}^{n} \varphi_{i}\left(X_{i}\right)\right]
$$

Logo,

$$
E_{f_{1}^{n}}\left[\prod_{i=1}^{n} \varphi_{i}\left(X_{i}\right)\right] \leq E_{f_{1}^{n}}\left[\prod_{i=1}^{k} \varphi_{i}\left(X_{i}\right)\right] E_{f_{1}^{n}}\left[\prod_{i=k+1}^{n} \varphi_{i}\left(X_{i}\right)\right]
$$

Finalizando com isto a prova de 2.1.5.

2.1.7 Corolário: Sob as condições de 2.1.5 (i.e., $\varphi_{i}$ concordantes.)

$$
E\left[\prod_{i=1}^{n} \varphi_{i}\left(X_{i}\right)\right] \leq \prod_{i=1}^{n} E\left[\varphi_{i}\left(X_{i}\right)\right.
$$

2.1.8 Teorema: Seja $\underline{X} S-M R R_{2}$, com respeito às funções $P F_{2}$. Sejam $\varphi_{i}^{*}$ funções $P F_{2}$, não necessariamente monótonas, $i=1, \cdots, k$ e sejam $\varphi_{i}$ funções $P F_{2}$ concordantes, para algum $k$, tal que $1 \leq k \leq n$. Então, para qualquer $l \geq 1$, tal que $k+l \leq n$,

$$
\begin{aligned}
E\left[\prod_{i=1}^{k} \varphi_{i}^{*}\left(X_{i}\right)\right. & \left.\prod_{i=k+1}^{n} \varphi_{i}\left(X_{i}\right)\right] E\left[\prod_{i=1}^{k} \varphi_{i}^{*}\left(X_{i}\right)\right] \\
& \leq E\left[\prod_{i=1}^{k} \varphi_{i}^{*}\left(X_{i}\right) \prod_{i=k+1}^{k+l} \varphi_{i}\left(X_{i}\right)\right] E\left[\prod_{i=1}^{k} \varphi_{i}^{*}\left(X_{i}\right) \prod_{i=k+l+1}^{n} \varphi_{i}\left(X_{i}\right) .\right.
\end{aligned}
$$

Prova de 2.1.8:

aplicando-se diretamente 2.1.5 à densidade

$$
\varphi\left(x_{k+1}, \cdots, x_{n}\right):=C^{-1} \int \cdots \int \varphi_{1}^{*}\left(x_{1}\right) \cdots \varphi_{k}^{*}\left(x_{k}\right) f\left(x_{1}, \cdots, x_{k}\right) d x_{1} \cdots d x_{k}
$$

onde $C:=E\left[\varphi_{1}^{*}\left(X_{1}\right) \cdots \varphi_{k}^{*}\left(X_{k}\right)\right][$ Karlin e $\operatorname{Rinott}(1980 \mathrm{~b}), \mathrm{pp} 505]$. Finalizando a prova de 2.1.8. 
Assim, a partir de 2.1 .5 chegamos na validade de 2.1.8, mas nosso objetivo é provar (e): f.d.p $S-M R R_{2} \Rightarrow N U O D$ e $N L O D$.

Notemos que, como as funções indicadoras de conjuntos na reta, são $P F_{2}$, podemos aplicar 2.1.8. Consideremos,

$$
\begin{aligned}
\varphi_{i}^{*}\left(X_{i}\right) & :=I_{\left[a_{i}, b_{i}\right]} i=1, \cdots, k, \\
\varphi_{j}\left(X_{j}\right) & :=I_{\left(-\infty, b_{j}\right]} j=k+1, \cdots, k+l, \\
\varphi_{s}\left(X_{s}\right) & :=I_{\left(-\infty, b_{s}\right]} s=k+l+1, \cdots, n .
\end{aligned}
$$

Logo, por 2.1.8,

$$
\begin{aligned}
& P\left(a_{i} \leq X_{i} \leq b_{i}, i \in I ; X_{\nu} \geq b_{\nu}, \nu \in J \cup K\right) P\left(a_{i} \leq X_{i} \leq b_{i}, i \in I\right) \\
& \quad \leq P\left(a_{i} \leq X_{i} \leq b_{i}, i \in I ; X_{\nu} \geq b_{\nu}, \nu \in J\right) P\left(a_{i} \leq X_{i} \leq b_{i}, i \in I ; X_{\nu} \geq b_{\nu}, \nu \in K\right) .
\end{aligned}
$$

Na desigualdade anterior pode-se substituir $X_{\nu} \geq b_{\nu}$ por $X_{\nu} \leq b_{\nu}$, para $\nu \in J \cup K$. Onde $I=\{1, \cdots, k\}, J=\{k+1, \cdots, k+l\}, K=\{k+l+1, \cdots, n\}$.

Como conseqüência, se $a_{i}=-\infty, b_{i}=+\infty, i \in I$,

$$
P\left(X_{\nu} \geq b_{\nu}, \nu \in J \cup K\right) \leq P\left(X_{\nu} \geq b_{\nu}, \nu \in J\right) P\left(X_{\nu} \geq b_{\nu}, \nu \in K\right),
$$

e progressivamente pode-se concluir que, se $\underline{X}=\left(X_{1}, \cdots, X_{n}\right)$ tem densidade $S$ $M R R_{2}$, então $\underline{X}$ é $N O D$ ( $\underline{X}$ é $N U O D$ e $\underline{X}$ é $\left.N L O D\right)$.

$$
\begin{aligned}
& \operatorname{NUOD} P\left(X_{i} \geq b_{i}, i=1, \cdots, n\right) \leq \prod_{i=1}^{n} P\left(X_{i} \geq b_{i}\right) \\
& N L O D P\left(X_{i} \leq a_{i}, i=1, \cdots, n\right) \leq \prod_{i=1}^{n} P\left(X_{i} \leq a_{i}\right)
\end{aligned}
$$

Concluindo-se finalmente com a prova de (e). 


\subsubsection{Corolário*}

$X_{1}, X_{2}$ têm f.d.p. conjunta $R R_{2} \Rightarrow X_{1}$ e $X_{2}$ são $N Q D \Leftrightarrow X_{1}$ e $X_{2}$ são $N A$.

Prova de 2.1.9:

notemos que no caso bivariado temos a equivalência entre os dois conceitos anteriores VUOD e $N L O D$, usualmente designado por $N Q D$. E $N Q D$ é equivalente a $N A$, Joag-Dev, Proscham(1982)] ou ponto (b) de 2.1.2.

Além disso, pelo ponto (e) de 2.1.4 ou por [Karlin e Rinott(1980 b)pp.505], concluimos que f.d.p. $R R_{2}$ implica $N Q D$. Concluíndo com a prova de 2.1.9.

Embora no caso bivariado os conceitos de $N U O D$ e $N L O D$ sejam equivalentes, isso pode não ser verificado quando a dimensão é maior [exemplo em EbrahimiGhosh (1981)]. 


\subsection{Critérios para examinar $\left(X_{1}, \cdots, X_{k}\right)$ com respeito à estrutura de associação entre suas variáveis}

\section{Objetivo}

Pesquisar em que condições é possível transformar uma estrutura de associação mista ( $P A$ e $N A$ em pares) numa estrutura homogênea $P A$ ou (excludente) $N A$.

Para realizar nosso objetivo, temos presente os seguintes fatos:

१ Se $\left(X_{1}, X_{2}\right)$ tem f.d.p. $f_{X_{1}, X_{2}}, T P_{2}$. Então, $X_{1}$ e $X_{2}$ são $P A$ [Joe(1997)].

ค Se $\left(X_{1}, X_{2}\right)$ tem f.d.p. $f_{X_{1}, X_{2}}, R R_{2}$. Então, $X_{1}$ e $X_{2}$ são $N A$ [Karlin e Rinott (1980 b); Proschan(1983)] ou 2.1.9.

Previamente enunciamos resultados e analisamos diversas situações. Em seguida Seção 4 - apresentamos as provas dos mesmos.

\subsubsection{Teorema*}

Sejam $\underline{X}=\left(X_{1}, X_{2}\right)$ com f.d.p. $f_{\underline{X}}, R R_{2}$ e $\underline{Y}=\eta(\underline{X})$, onde $\eta\left(x_{1}, x_{2}\right)=$ $\left(\eta_{1}\left(x_{1}\right), \eta_{2}\left(x_{2}\right)\right)$, com coordenadas $\eta_{1}, \eta_{2}$ diferenciáveis inversíveis e discordantes (ou seja, quando uma coordenada cresce a outra decresce). Então, a f.d.p. de $\underline{Y}, f_{\underline{Y}}$, é $T P_{2}$. Conseqüentemente, $\underline{Y}$ é $P A$. 
2.2.2 Examinemos $\left(X_{1}, \cdots, X_{k}\right)$ com variáveis satisfazendo a condição de $P A$ ou NA em pares, quando ambas as condições decorrem dos dois primeiros fatos destacados previamente.

Afirmação

Caso 1: Se $X_{1}$ é a variável a predizer a partir de $\left(X_{2}, \cdots, X_{k}\right)$ e

$$
X_{1}, X_{s} P A, s \neq j_{0}, s=2, \cdots, k ; \quad X_{1}, X_{j_{0}} N A,
$$

podemos construir uma variável $\eta_{j_{0}}\left(X_{j_{0}}\right)$, tal que,

$$
X_{s}, \eta_{j_{0}}\left(X_{j_{0}}\right) P A, 1 \leq s<j_{0} ; \quad \eta_{j_{0}}\left(X_{j_{0}}\right), X_{s} P A, \quad j_{0}+1 \leq s \leq k
$$

De modo semelhante,

Caso 2: Se $X_{j_{0}}$ é a variável a predizer a partir de $\left(X_{1}, \cdots, X_{j_{0}-1}, X_{j_{0}+1}, X_{k}\right)$ e

$$
\begin{array}{rl}
X_{s}, X_{j_{0}} & N A, s=1, \cdots, j_{0}-1 ; \\
X_{j_{0}}, X_{t} \quad N A, t & =j_{0}+1, \cdots, k ; \\
X_{i}, X_{j} \quad P A, i & \neq j_{0} \neq j, i \neq j,
\end{array}
$$

podemos construir $k-1$ variáveis $\eta_{i}\left(X_{i}\right), i \neq j_{0}$, tais que

$$
\begin{aligned}
\eta_{i}\left(X_{i}\right), \eta_{j}\left(X_{j}\right) \quad P A, \forall i, j & \neq j_{0} \\
\eta_{i}\left(X_{i}\right), X_{j_{0}} P A, i & =1, \cdots, j_{0}-1 ; \\
X_{j_{0}}, \eta_{j}\left(X_{j}\right) P A, j & =j_{0}+1, \cdots, k .
\end{aligned}
$$

Em ambos casos, $X_{j_{0}}$ é a variável "responsável" pela negatividade no vetor aleatório. Notemos que o objetivo, na afirmação prévia é obter uma estrutura positiva - esta 
estrutura foi mais pesquisada e oferece certas vantagens em relação à estrutura negativa.

$\operatorname{Em}\left(X_{1}, \cdots, X_{k}\right), X_{1}$ é a variável a predizer

\subsubsection{Teorema*}

Seja $\left(X_{1}, \cdots, X_{j_{0}}, \cdots, X_{k}\right)$ um vetor aleatório, tal que as densidades marginais $f_{i, j}$ são $T P_{2} \forall j \neq j_{0}, j=1, \cdots, k$; e $f_{1, j_{0}}$ é $R R_{2}$.

Suponhamos ainda que para $2 \leq s<j_{0}, j_{0}+1 \leq t \leq k$, as marginais $f_{1, s, j_{0}}$ e $f_{1, j_{0}, t}$ verificam:

(i) $f_{1, s, j_{0}}\left(x_{1}, x_{s}, x_{j_{0}}\right) f_{1, s, j_{0}}\left(x_{1}^{\prime}, x_{s}^{\prime}, x_{j_{0}}^{\prime}\right)$

$\leq f_{1, s, j_{0}}\left(x_{1} \vee x_{1}^{\prime}, x_{s} \vee x_{s}^{\prime}, x_{j_{0}} \wedge x_{j_{0}}^{\prime}\right) f_{1, s, j_{0}}\left(x_{1} \wedge x_{1}^{\prime}, x_{s} \wedge x_{s}^{\prime}, x_{j_{0}} \vee x_{j_{0}}^{\prime}\right)$,

(ii) $f_{1, j_{0}, t}\left(x_{1}, x_{j_{0}}, x_{t}\right) f_{1, j_{0}, t}\left(x_{1}^{\prime}, x_{j_{0}}^{\prime}, x_{t}^{\prime}\right)$

$\leq f_{1, j_{0}, t}\left(x_{1} \vee x_{1}^{\prime}, x_{j_{0}} \wedge x_{j_{0}}^{\prime}, x_{t} \vee x_{t}^{\prime}\right) f_{1, j_{0}, t}\left(x_{1} \wedge x_{1}^{\prime}, x_{j_{0}} \vee x_{j_{0}}^{\prime}, x_{t} \wedge x_{t}^{\prime}\right)$,

respectivamente. Nessas condições, se $\eta_{j_{0}}: R \rightarrow R$ é decrescente, inversível e diferenciável, então

$$
\begin{array}{lll}
(a)\left(X_{s}, \eta_{j_{0}}\left(X_{j_{0}}\right)\right) & \text { tem f.d.p. } & f_{X_{s}, \eta_{j_{0}}\left(X_{j_{0}}\right)}, T P_{2}, \quad 1 \leq s<j_{0} \\
(b)\left(\eta_{j_{0}}\left(X_{j_{0}}\right), X_{t}\right) & \text { tem f.d.p. } \quad f_{\eta_{j_{0}}\left(X_{j_{0}}\right), X_{t}}, T P_{2}, \quad j_{0}+1 \leq t \leq k
\end{array}
$$

Notação:

$$
f_{i, j, k}\left(x_{1}, x_{j}, x_{k}\right):=f_{X_{i}, X_{j}, X_{k}}\left(x_{1}, x_{j}, x_{k}\right), f_{i, j}\left(x_{1}, x_{j}\right)=f_{X_{i}, X_{j}}\left(x_{1}, x_{j}\right) .
$$


2.2.4 Corolário* Seja $\left(X_{1}, \cdots, X_{j_{0}}, \cdots, X_{k}\right)$ um vetor aleatório, tal que as densidades marginais $f_{i, j}$ satisfazem,

$f_{s, j_{0}} R R_{2}, \forall 1 \leq s<j_{0} . \quad f_{j_{0}, t} R R_{2}, \forall j_{0}<t \leq k, f_{1, j} T P_{2}, \forall j \neq j_{0}, j=1, \cdots, k$.

Nessas condições, se $\eta_{j_{0}}: R \rightarrow R$ é decrescente, inversível e diferenciável, então

$$
\begin{aligned}
& (a)\left(X_{s}, \eta_{j_{0}}\left(X_{j_{0}}\right)\right) \text { tem f.d.p. } \quad f_{X_{s}, \eta_{j_{0}}\left(X_{j_{0}}\right), T P_{2}, \quad 1 \leq s<j_{0}} \\
& (b)\left(\eta_{j_{0}}\left(X_{j_{0}}\right), X_{t}\right) \quad \text { tem f.d.p. } \quad f_{\eta_{j_{0}}\left(X_{j_{0}}\right), X_{t}}, T P_{2}, \quad j_{0}+1 \leq t \leq k .
\end{aligned}
$$

\subsubsection{Teorema*}

Seja $\underline{X}=\left(X_{1}, \cdots, X_{J_{0}}, \cdots, X_{k}\right)$ com f.d.p.

$$
f_{X_{1}, \cdots, X_{j_{0}}, \cdots, X_{k}}\left(x_{1}, \cdots, x_{j_{0}}, \cdots, x_{k}\right)=h\left(x_{1}, \cdots, x_{j_{0}-1}, x_{j_{0}+1}, \cdots, x_{k}\right) K\left(x_{1}, x_{j_{0}}\right),
$$

onde $K$ é $R R_{2}$, e $h$ satisfaz a seguinte condição:

$$
\begin{aligned}
\text { (iii) } \int h\left(u, x_{(2)}^{(s-1)} \cdot v, x_{(s+1)}^{(k)}\right) d\left(x_{(2)}^{(s-1)}, x_{(s+1)}^{(k)}\right) \quad & \text { é } \quad T P_{2} \text { nas v.a. } X_{1}, X_{s}, \\
\forall 3 \leq s<j_{0} & \text { ou } \quad j_{0}+1 \leq s<k-1 \\
\int h\left(u, x_{(2)}^{(k-1)}, v\right) d\left(x_{(2)}^{(k-1)}\right) & \text { é } \quad T P_{2} \text { nas v.a. } X_{1}, X_{k} .
\end{aligned}
$$

Então,

$$
f_{X_{1}, X_{s}} \text { é } T P_{2}, \forall s \neq j_{0}, s=2, \cdots, k \text { e } f_{X_{1}, X_{\jmath_{0}}} \text { é } R R_{2} \text {. }
$$

Nesse contexto, se $\eta_{j_{0}}: R \rightarrow R$ é decrescente, inversível e diferenciável; então,

(a) $\left(X_{s}, \eta_{j_{0}}\left(X_{j_{0}}\right)\right)$ tem f.d.p. $f_{X_{s}, \eta_{j_{0}}\left(X_{j_{0}}\right)}, \quad T P_{2}, \quad 1 \leq s<j_{0}$,

(b) $\left(\eta_{j_{0}}\left(X_{j_{0}}\right), X_{s}\right)$ tem f.d.p. $f_{\eta_{j_{0}}\left(X_{j_{0}}\right), X_{s}}, \quad T P_{2}, \quad j_{0}+1 \leq s \leq k$.

Onde $x_{(i)}^{(j)}=\left(x_{i}, x_{i+1}, \cdots, x_{j}\right)$ 
2.3. Mistura de associações:

\section{Vetores Aleatórios contendo os dois Tipos de Associações}

\subsubsection{Exemplo}

Se $\left(X_{1}, X_{2}, X_{3}, X_{4}\right)$ tem distribuição $N_{4}(\underline{0}, \Sigma)$, onde

$$
\Sigma=\left(\begin{array}{rrrr}
0.80 & 0.26 & -0.05 & 0.42 \\
& 3.16 & 0.00 & 1.63 \\
& & 1.58 & 0.00 \\
& & & 2.74
\end{array}\right)
$$

a distribuição de $\underline{X}$ pode ser expressa como produto de uma $N_{3}\left(\underline{0}, \Sigma_{1}^{*}\right)$ com uma $N_{2}\left(\underline{0}, \Sigma_{2}^{*}\right)$,

$$
\text { onde } \Sigma_{1}^{*}=\left(\begin{array}{rrr}
1.50 & 0.50 & 0,80 \\
& 1.50 & 0,90 \\
& & 1.50
\end{array}\right), \Sigma_{2}^{*}=\left(\begin{array}{rr}
1.00 & -0.05 \\
& 1.24
\end{array}\right)
$$

e

$$
\begin{gathered}
\left(X_{1}, X_{2}, X_{4}\right) \text { tem distribuição } N_{3}\left(\underline{0}, \Sigma_{1}^{*}\right), M T P_{2} \\
\left(X_{1}, X_{3}\right) \text { tem distribuição } N_{2}\left(\underline{0}, \Sigma_{2}^{*}\right), R R_{2} .
\end{gathered}
$$

O exemplo anterior motivou a seguinte proposição.

\subsubsection{Proposição*}

Seja $\underline{X}=\left(X_{1}, \cdots, X_{J_{0}}, \cdots, X_{k}\right)$ com f.d.p.

$$
f_{X_{1}, \cdots, X_{j_{0}}, \cdots, X_{k}}\left(x_{1}, \cdots, x_{j_{0}}, \cdots, x_{k}\right)=h\left(x_{1}, \cdots, x_{j_{0}-1}, x_{j_{0}+1}, \cdots, x_{k}\right) K\left(x_{1}, x_{j_{0}}\right)
$$

onde $K$ é $R R_{2}$ e $h$ é $M T P_{2}$; então,

$$
f_{X_{1}, X_{s}} \text { é } T P_{2}, \forall s \neq j_{0}, s=1, \cdots, k \text { e } f_{X_{1}, X_{\jmath_{0}}} \text { é } R R_{2} \text {. }
$$


Nesse contexto, se $\eta_{j_{0}}: R \rightarrow R$ é decrescente, inversível e diferenciável, então,

$$
\begin{aligned}
& (a)\left(X_{s}, \eta_{j_{0}}\left(X_{j_{0}}\right)\right) \text { tem f.d.p. } f_{X_{s}, \eta_{j_{0}}\left(X_{j_{0}}\right)}, T P_{2}, \quad 1 \leq s<j_{0} \\
& (b)\left(\eta_{j_{0}}\left(X_{j_{0}}\right), X_{s}\right) \text { tem f.d.p. } f_{\eta_{j_{0}}\left(X_{j_{0}}\right), X_{s}}, T P_{2}, \quad j_{0}+1 \leq s \leq k . \\
& \text { 2.4. Complementos das SeçÕes } 2 \text { e } 3
\end{aligned}
$$

A seguir, apresentamos as provas dos resultados anteriores e outros complementares.

\subsubsection{Prova: de 2.2.1.}

Consideremos $y_{1}^{\prime} \leq y_{1}, y_{2} \leq y_{2}^{\prime}$. Precisamos verificar

$$
f_{\underline{Y}}\left(y_{1}, y_{2}\right) f_{\underline{Y}}\left(y_{1}^{\prime}, y_{2}^{\prime}\right) \leq f_{\underline{Y}}\left(y_{1}^{\prime}, y_{2}\right) f_{\underline{Y}}\left(y_{1}, y_{2}^{\prime}\right)
$$

e sendo $y_{i}=\eta_{i}\left(x_{i}\right), y_{i}^{\prime}=\eta_{i}\left(x_{i}^{\prime}\right), i=1,2$. A desigualdade a verificar é equivalente a $f_{\underline{X}}\left(\eta_{1}^{-1}\left(y_{1}\right), \eta_{2}^{-1}\left(y_{2}^{\prime}\right)\right) f_{\underline{X}}\left(\eta_{1}^{-1}\left(y_{1}^{\prime}\right), \eta_{2}^{-1}\left(y_{2}\right)\right) \geq f_{\underline{X}}\left(\eta_{1}^{-1}\left(y_{1}\right), \eta_{2}^{-1}\left(y_{2}\right)\right) f_{\underline{X}}\left(\eta_{1}^{-1}\left(y_{1}^{\prime}\right), \eta_{2}^{-1}\left(y_{2}^{\prime}\right)\right)$ Suponhamos sem perda da generalidade que $\eta_{1}$ é monótona decrescente, inversível e portanto com inversa monótona decrescente; $\eta_{2}$ monótona crescente, inversível e portanto com inversa monótona crescente. Assim, como $y_{1}^{\prime} \leq y_{1}$ e $y_{2} \leq y_{2}^{\prime}$,

$$
\begin{aligned}
& \eta_{1}^{-1}\left(y_{1}\right) \leq \eta_{1}^{-1}\left(y_{1}^{\prime}\right) \\
& \eta_{2}^{-1}\left(y_{2}\right) \leq \eta_{2}^{-1}\left(y_{2}^{\prime}\right)
\end{aligned}
$$

e, finalmente, como $f_{\underline{X}}$ é $R R_{2}$, temos a desigualdade. 
2.4.2 Proposição* Seja $\underline{Y}$ com f.d.p. $f_{\underline{Y}} \cdot f_{\underline{Y}} M T P_{2}$ e $f_{2}>_{T P_{2}} f_{1}, f_{1}$ monótona crescente não negativas, então, $\operatorname{Cov}\left(f_{1}(\underline{Y}), f_{2}(\underline{Y})\right) \geq 0$.

Prova de 2.4.2:

$$
\begin{aligned}
& \text { (1) } f_{\underline{Y}} M T P_{2} \Leftrightarrow f_{\underline{Y}}(\underline{u}) f_{\underline{Y}}(\underline{v}) \leq f_{\underline{Y}}(\underline{u} \vee \underline{v}) f_{\underline{Y}}(\underline{u} \wedge \underline{v}) \\
& (2) f_{2}>_{T P_{2}} f_{1} \Leftrightarrow f_{1}(\underline{u}) f_{2}(\underline{v}) \leq f_{2}(\underline{u} \vee \underline{v}) f_{1}(\underline{u} \wedge \underline{v}) \\
& \text { (3) } f_{1} \text { monótona crescente } \Rightarrow f_{1}(\underline{u} \wedge \underline{v}) \leq f_{1}(\underline{u} \vee \underline{v})
\end{aligned}
$$

a partir de (1)(2) e (3),

$$
f_{1}(\underline{u}) f_{\underline{Y}}(\underline{u}) f_{2}(\underline{v}) f_{\underline{Y}}(\underline{v}) \leq f_{1}(\underline{u} \vee \underline{v}) f_{2}(\underline{u} \vee \underline{v}) f_{\underline{Y}}(\underline{u} \vee \underline{v}) f_{\underline{Y}}(\underline{u} \wedge \underline{v})
$$

Definamos,

$$
\begin{aligned}
g_{i}(\cdot) & :=f_{i}(\cdot) f_{\underline{Y}}(\cdot) i=1,2, \\
g_{3}(\cdot) & :=f_{\underline{Y}}(\cdot) \\
g_{4}(\cdot) & :=f_{1}(\cdot) f_{2}(\cdot) f_{\underline{Y}}(\cdot) .
\end{aligned}
$$

Temos que,

$$
g_{1}(\underline{u}) g_{2}(\underline{v}) \leq g_{3}(\underline{u} \wedge \underline{v}) g_{4}(\underline{u} \vee \underline{v})
$$

então, [Karlim e Rinott(1980 a)]permitem garantir a seguinte desigualdade,

$$
\int g_{1}(\underline{y}) d \underline{y} \int g_{2}(\underline{y}) d \underline{y} \leq \int g_{3}(\underline{y}) d \underline{y} \int g_{4}(\underline{y}) d \underline{y}
$$

equivalentemente,

$$
E_{\underline{Y}}\left(f_{1}\right) E_{\underline{Y}}\left(f_{2}\right) \leq E_{\underline{Y}}\left(f_{1} f_{2}\right)
$$

i.e.,

$$
\operatorname{Cov}\left(f_{1}(\underline{Y}), f_{2}(\underline{Y})\right) \geq 0 .
$$




\subsubsection{Teorema*}

Consideremos $\left(X_{1}, \cdots, X_{4}\right)$ tais que as densidades marginais $f_{X_{1}, X_{2}}$ e $f_{X_{1}, X_{4}}$ são $T P_{2}$, enquanto que $f_{X_{1}, X_{3}}$ é $R R_{2}$.

Se $\eta_{3}: R \rightarrow R$ é decrescente, inversível, diferenciável e $f_{X_{1}, X_{2}, X_{3}}, f_{X_{1}, X_{3}, X_{4}}$ verificam.

(i) $f_{X_{1}, X_{2}, X_{3}}\left(x_{1}, x_{2}, x_{3}\right) f_{X_{1}, X_{2}, X_{3}}\left(x_{1}^{\prime}, x_{2}^{\prime}, x_{3}^{\prime}\right)$

$$
\leq f_{X_{1}, X_{2}, X_{3}}\left(x_{1} \vee x_{1}^{\prime}, x_{2} \vee x_{2}^{\prime}, x_{3} \wedge x_{3}^{\prime}\right) f_{X_{1}, X_{2}, X_{3}}\left(x_{1} \wedge x_{1}^{\prime}, x_{2} \wedge x_{2}^{\prime}, x_{3} \vee x_{3}^{\prime}\right),
$$

(ii) $f_{X_{1}, X_{3}, X_{4}}\left(x_{1}, x_{3}, x_{4}\right) f_{X_{1}, X_{3}, X_{4}}\left(x_{1}^{\prime}, x_{3}^{\prime}, x_{4}^{\prime}\right)$

$$
\leq f_{X_{1}, X_{3}, X_{4}}\left(x_{1} \vee x_{1}^{\prime}, x_{3} \wedge x_{3}^{\prime}, x_{4} \vee x_{4}^{\prime}\right) f_{X_{1}, X_{3}, X_{4}}\left(x_{1} \wedge x_{1}^{\prime}, x_{3} \vee x_{3}^{\prime}, x_{4} \wedge x_{4}^{\prime}\right)
$$

respectivamente, então,

$$
\begin{array}{lll}
(a)\left(X_{1}, \eta_{3}\left(X_{3}\right)\right) & \text { tem f.d.p. } & f_{X_{1}, \eta_{3}\left(X_{3}\right)}, T P_{2}, \\
(b)\left(X_{2}, \eta_{3}\left(X_{3}\right)\right) & \text { tem f.d.p. } & f_{X_{2}, \eta_{3}\left(X_{3}\right)}, T P_{2}, \\
(c)\left(\eta_{3}\left(X_{3}\right), X_{4}\right) & \text { tem f.d.p. } & f_{\eta_{3}\left(X_{3}\right), X_{4}}, T P_{2} .
\end{array}
$$

Prova de 2.4.3:

a prova de $(a)$ decorre de 2.2.1. Como as provas de $(b)$ e $(c)$ são análogas, então provemos 
(b) : $f_{X_{2}, \eta_{3}\left(X_{3}\right)}$ é $T P_{2}$ se, e somente se,

$$
\begin{aligned}
& f_{X_{2}, \eta_{3}\left(X_{3}\right)}\left(x_{2}, \eta_{3}\left(x_{3}\right)\right) f_{X_{2}, \eta_{3}\left(X_{3}\right)}\left(x_{2}^{\prime}, \eta_{3}\left(x_{3}^{\prime}\right)\right) \\
& \quad \leq f_{X_{2}, \eta_{3}\left(X_{3}\right)}\left(x_{2} \vee x_{2}^{\prime}, \eta_{3}\left(x_{3}\right) \vee \eta_{3}\left(x_{3}^{\prime}\right)\right) f_{X_{2}, \eta_{3}\left(X_{3}\right)}\left(x_{2} \wedge x_{2}^{\prime}, \eta_{3}\left(x_{3}\right) \wedge \eta_{3}\left(x_{3}^{\prime}\right)\right) .
\end{aligned}
$$

O caso de interesse é quando $x_{2} \leq x_{2}^{\prime}, \quad \eta_{3}\left(x_{3}^{\prime}\right) \leq \eta_{3}\left(x_{3}\right)$ (i.e. $x_{3} \leq x_{3}^{\prime}$ ) a desigualdade a verificar fica,

$f_{X_{2}, \eta_{3}\left(X_{3}\right)}\left(x_{2}, \eta_{3}\left(x_{3}\right)\right) f_{X_{2}, \eta_{3}\left(X_{3}\right)}\left(x_{2}^{\prime}, \eta_{3}\left(x_{3}^{\prime}\right)\right) \leq f_{X_{2}, \eta_{3}\left(X_{3}\right)}\left(x_{2}^{\prime}, \eta_{3}\left(x_{3}\right)\right) f_{X_{2}, \eta_{3}\left(X_{3}\right)}\left(x_{2}, \eta_{3}\left(x_{3}^{\prime}\right)\right)$, equivalentemente,

$$
f_{X_{2}, X_{3}}\left(x_{2}, x_{3}\right) f_{X_{2}, X_{3}}\left(x_{2}^{\prime}, x_{3}^{\prime}\right) \leq f_{X_{2}, X_{3}}\left(x_{2}^{\prime}, x_{3}\right) f_{X_{2}, X_{3}}\left(x_{2}, x_{3}^{\prime}\right)
$$

ou

$$
\begin{aligned}
& \int f_{X_{1}, X_{2}, X_{3}}\left(x_{1}, x_{2}, x_{3}\right) d x_{1} \int f_{X_{1}, X_{2}, X_{3}}\left(x_{1}, x_{2}^{\prime}, x_{3}^{\prime}\right) d x_{1} \\
\leq & \int f_{X_{1}, X_{2}, X_{3}}\left(x_{1}, x_{2}^{\prime}, x_{3}\right) d x_{1} \int f_{X_{1}, X_{2}, X_{3}}\left(x_{1}, x_{2}, x_{3}^{\prime}\right) d x_{1},
\end{aligned}
$$

e por hipótese,

(i) $f_{X_{1}, X_{2}, X_{3}}\left(u, x_{2}, x_{3}\right) f_{X_{1}, X_{2}, X_{3}}\left(v, x_{2}^{\prime}, x_{3}^{\prime}\right)$

$$
\leq f_{X_{1}, X_{2}, X_{3}}\left(u \vee v, x_{2} \vee x_{2}^{\prime}, x_{3} \wedge x_{3}^{\prime}\right) f_{X_{1}, X_{2}, X_{3}}\left(u \wedge v, x_{2} \wedge x_{2}^{\prime}, x_{3} \vee x_{3}^{\prime}\right)
$$

ou analogamente,

$f_{X_{1}, X_{2}, X_{3}}\left(u, x_{2}, x_{3}\right) f_{X_{1}, X_{2}, X_{3}}\left(v, x_{2}^{\prime}, x_{3}^{\prime}\right) \leq f_{X_{1}, X_{2}, X_{3}}\left(u \vee v, x_{2}^{\prime}, x_{3}\right) f_{X_{1}, X_{2}, X_{3}}\left(u \wedge v, x_{2}, x_{3}^{\prime}\right)$.

Logo, por 1.3.1 vale a desigualdade que envolve as integrais. Assim, resulta $(b)$ : $f_{X_{2}, \eta_{3}\left(X_{3}\right)} T P_{2}$. 


\section{Comentário}

É possível verificar que as condições (i) e (ii) são compatíveis com as condições seguintes,

$$
\begin{aligned}
& \left(X_{1}, X_{2}\right) \text { com f.d.p. } f_{X_{1}, X_{2}}, T P_{2} \text {, } \\
& \left(X_{1}, X_{4}\right) \text { com f.d.p. } f_{X_{1}, X_{4}}, T P_{2} \text {, } \\
& \left(X_{1}, X_{3}\right) \text { com f.d.p. } f_{X_{1}, X_{3}}, R R_{2} \text {. }
\end{aligned}
$$

\subsubsection{Corolário*}

Consideremos $\left(X_{1}, \cdots, X_{4}\right)$ tais que $f_{X_{s}, X_{3}}$ é $R R_{2}$ para $s=1,2, f_{X_{3}, X_{4}}$ é $R R_{2}$, enquanto $f_{X_{1}, X_{i}}$ é $T P_{2}$ para $i=2,4$.

Se $\eta_{3}: R \rightarrow R$ é decrescente, inversível e diferenciável, então ,

$$
\begin{array}{lll}
(a)\left(X_{1}, \eta_{3}\left(X_{3}\right)\right) & \text { tem f.d.p. } & f_{X_{1}, \eta_{3}\left(X_{3}\right)}, T P_{2}, \\
(b)\left(X_{2}, \eta_{3}\left(X_{3}\right)\right) & \text { tem f.d.p. } & f_{X_{2}, \eta_{3}\left(X_{3}\right)}, T P_{2}, \\
(c)\left(\eta_{3}\left(X_{3}\right), X_{4}\right) & \text { tem f.d.p. } & f_{\eta_{3}\left(X_{3}\right), X_{4}}, T P_{2} .
\end{array}
$$

Conseqüentemente, vale 2.2 .3 e 2.2 .4 .

Comentário: Podemos dizer que o causante da negatividade no vetor aleatório é a variável $X_{3}\left(X_{j_{0}}\right)$. Assim, ante uma única variável responsável pela megatividade no vetor, podemos obter un vetor aleatório, de estrutura positiva, "equivalente" ao dado inicialmente. 
2.4.5 Prova: de 2.2.5.

Precisamos ver que a distribuição verifica as condições $(i)$ e (ii) de 2.2.3, e que $f_{X_{1}, X_{s}}$ é $T P_{2} \forall s \neq j_{0}, s=1, \cdots, k$; enquanto que $f_{X_{1}, X_{j_{0}}}$ é $R R_{2}$.

Para a última questão, é só lembrar que passo a passo devemos fixar as $k-2$ variáveis restantes, e que $h$ independe de $X_{j_{0}}$.

A partir daí, e tendo presente que $\eta_{j_{0}}$ é decrescente, inversível e diferenciável. pode-se concluir a validez de $(a)$. Análogo procedimento para provar (b).

Para provar a validade da condição $(i)$ de 2.2 .3 , notemos que,

$$
f_{X_{1}, X_{s}, X_{j_{0}}}\left(x_{1}, x_{s}, x_{j_{0}}\right)=K\left(x_{1}, x_{j_{0}}\right) \int h\left(x_{1}, x_{(2)}^{(s-1)}, x_{s}, x_{(s+1)}^{(k)}\right) d\left(x_{(2)}^{(s-1)}, x_{(s+1)}^{(k)}\right) .
$$

Como $K$ é $R R_{2}$,

$$
K\left(x_{1}, x_{j_{0}}\right) K\left(x_{1}^{\prime}, x_{j_{0}}^{\prime}\right) \leq K\left(x_{1}^{\prime}, x_{j_{0}}\right) K\left(x_{1}, x_{j_{0}}^{\prime}\right), \text { qdo } x_{1} \leq x_{1}^{\prime}, x_{j_{0}} \leq x_{j_{0}}^{\prime}
$$

Equivalentemente,

$$
(1) K\left(x_{1}, x_{j_{0}}\right) K\left(x_{1}^{\prime}, x_{j_{0}}^{\prime}\right) \leq K\left(x_{1} \vee x_{1}^{\prime}, x_{j_{0}} \wedge x_{j_{0}}^{\prime}\right) K\left(x_{1} \wedge x_{1}^{\prime}, x_{j_{0}} \vee x_{j_{0}}^{\prime}\right)
$$

A hipótese (iii) permite concluir a prova, pois,

(2) $\int h\left(x_{1}, x_{(2)}^{(s-1)}, x_{s}, x_{(s+1)}^{(k)}\right) d\left(x_{(2)}^{(s-1)}, x_{(s+1)}^{(k)}\right) \int h\left(x_{1}^{\prime}, x_{(2)}^{(s-1)}, x_{s}^{\prime}, x_{(s+1)}^{(k)}\right) d\left(x_{(2)}^{(s-1)}, x_{(s+1)}^{(k)}\right)$

$$
\begin{gathered}
\leq \int h\left(x_{1} \vee x_{1}^{\prime}, x_{(2)}^{(s-1)}, x_{s} \vee x_{s}^{\prime}, x_{(s+1)}^{(k)}\right) d\left(x_{(2)}^{(s-1)}, x_{(s+1)}^{(k)}\right) \\
\int h\left(x_{1} \wedge x_{1}^{\prime}, x_{(2)}^{(s-1)}, x_{s} \wedge x_{s}^{\prime}, x_{(s+1)}^{(k)}\right) d\left(x_{(2)}^{(s-1)}, x_{(s+1)}^{(k)}\right) .
\end{gathered}
$$


Fazendo o produto de (1) e (2) temos que $f_{X_{1}, X_{s}, X_{j_{0}}}$ verifica a condição $(i)$ de 2.2.3, i.e..

$$
\begin{aligned}
& \text { (i) } f_{X_{1}, X_{s}, X_{j_{0}}}\left(x_{1}, x_{s}, x_{j_{0}}\right) f_{X_{1}, X_{s}, X_{j_{0}}}\left(x_{1}^{\prime}, x_{s}^{\prime}, x_{j_{0}}^{\prime}\right) \\
& \quad \leq f_{X_{1}, X_{s}, X_{j 0}}\left(x_{1} \vee x_{1}^{\prime}, x_{s} \vee x_{s}^{\prime}, x_{j_{0}} \wedge x_{j_{0}}^{\prime}\right) f_{X_{1}, X_{s}, X_{j_{0}}}\left(x_{1} \wedge x_{1}^{\prime}, x_{s} \wedge x_{s}^{\prime}, x_{j_{0}} \vee x_{j_{0}}^{\prime}\right)
\end{aligned}
$$

Isso completa a prova para $2 \leq s<j_{0}$, e, com um procedimento análogo tomando $j_{0}+1 \leq t \leq k$. pode-se verificar que $f_{X_{1}, X_{j_{0}}, X_{t}}$ cumpre a condição (ii), de 2.2.3.

Para a prova de 2.3.2 é suficente notar que como $h$ é $M T P_{2}$, então, verifica-se a condição (iii) de 2.2.5.

As técnicas percorridas, tanto quanto o uso de resultados da natureza de [Karlin e Rinott (1980 a)], são novamente explorados, na seção 2 do apêndice. 


\subsection{Herança da condição $P A$}

\section{Afirmação}

Se temos $\left(X_{1}, \cdots, X_{k}\right), k \geq 3$, vetor aleatório $P A, f: R^{k-1} \rightarrow R$, crescente, então, $X_{1}$ e $f\left(X_{2}, \cdots, X_{k}\right)$ são variáveis aleatórias $P A$.

Apresentamos e provamos alguns resultados presentes em [Barlow e Proschan, Statistical Theory of Reliability and Life Testing (1981)pp.30].

\subsubsection{Proposição}

$\underline{T}=\left(T_{1}, \cdots, T_{n}\right)$ é $P A$ (onde cada variável aleatória não é necesariamente binária) se, e somente se, para todo par de funções binárias e crescentes $\Gamma$ e $\Delta$,

$$
\operatorname{Cov}\{\Gamma(\underline{T}), \Delta(\underline{T})\} \geq 0
$$

A propriedade anterior é verificada a partir dos seguintes exercícios.

2.5.2 Exercício : Sejam $S$ e $T$ variáveis aleatórias com $E(S), E(T), E(S T)$ finitas. Dados $s$ e $t$ valores arbitrários na imagem das variáveis $S$ e $T$ respectivamente, consideremos $X_{S}(s):=I_{\{S>s\}}$ e $X_{T}(t):=I_{\{T>t\}}$ como as respectivas funções indicadoras dos conjuntos $\{S>s\}$ e $\{T>t\}$. Então,

$$
\operatorname{Cov}\{S, T\}=\int_{-\infty}^{\infty} \int_{-\infty}^{\infty} \operatorname{Cov}\left\{X_{S}(s), X_{T}(t)\right\} d s d t
$$


2.5.3 Exercício : Se $\underline{T}$ é $P A$ e $f, g$ são funções crescentes, então

$$
\operatorname{Cov}\{f(\underline{T}), g(\underline{T}))\} \geq 0
$$

assumindo que a covariância existe.

Prova de 2.5.1

$\Rightarrow$ : evidente.

$\Leftarrow$ : Notemos que se $f$ e $g$ são funções crescentes arbitrárias, $f(\underline{T})$ e $g(\underline{T})$ são variáveis aleatórias, cujas imagens podem estar em todo $R$ ou num subconjunto dele. Se consideramos as funções $X_{f(\underline{T})}(s)$ e $X_{g(\underline{T})}(t)$, elas são funções crescentes binárias de $\underline{T}$. Definindo,

$$
S:=f(\underline{T}), T:=g(\underline{T}), \Gamma_{s}(\underline{T}):=X_{f(\underline{T})}(s), \Delta_{t}(\underline{T}):=X_{g(\underline{T})}(t),
$$

como, para cada par de valores fixos $s$ e $t$ as funções $\Gamma_{s}$ e $\Delta_{t}$ são funções crescentes e binárias, então,

$$
\operatorname{Cov}\left\{\Gamma_{s}(\underline{T}), \Delta_{t}(\underline{T})\right\} \geq 0
$$

por hipótese. Finalmente, pelo Exercício 5.2 resulta

$$
\operatorname{Cov}\{f(\underline{T}), g(\underline{T})\} \geq 0
$$

para $f$ e $g$ funções crescentes e arbitrárias. Imediatamente - por definição - $\underline{T}$ é $P A$. 
2.5.4 Proposição: Funções crescentes de variáveis aleatórias $P A$ são $P A$.

Prova: Consideremos $\underline{T}=\left(T_{1}, \cdots, T_{n}\right)$ um vetor $P A$, e uma seqüência de $m$ funções crescentes $f_{i}: R^{n} \rightarrow R, i=1, \cdots, m$ com $m \neq n$ em geral. Definamos as variáveis aleatórias $S_{i}:=f_{i}(\underline{T})$, o vetor $\underline{S}:=\left(S_{1}, \cdots, S_{m}\right)$ tem coordenadas que em geral são combinação de todas as variáveis aleatórias $P A,\left\{T_{i}\right\}_{i=1}^{n}$. Sejam $\Gamma, \Delta: R^{m} \rightarrow R$ funções crescentes, binárias e arbitrárias. Logo,

$$
\begin{gathered}
\Gamma(f(\underline{T})):=\Gamma\left(f_{1}(\underline{T}), \cdots, f_{m}(\underline{T})\right) \mathrm{e} \\
\Delta(f(\underline{T})):=\Delta\left(f_{1}(\underline{T}), \cdots, f_{m}(\underline{T})\right)
\end{gathered}
$$

são funções crescentes binárias, como funções de $\underline{T}$. Assim,

$$
\operatorname{Cov}_{\underline{S}}\{\Gamma(\underline{S}), \Delta(\underline{S})\}=\operatorname{Cov}_{\underline{T}}\{\Gamma(f(\underline{T})), \Delta(f(\underline{T}))\} \geq 0
$$

pois, $\underline{T}$ é $P A$ e $\Gamma \circ f, \Delta \circ f$ são crescentes binárias. Portanto, pela arbitrariedade das funções $\Gamma$ e $\Delta$ e a afirmação precedente, temos, $\underline{S} P A$.

\subsubsection{Aplicação}

Se $\left(X_{1}, X_{2}, X_{3}\right)$ é $P A, f_{i}: R^{3} \rightarrow R, i=1,2$, onde $f_{1}(u, v, w)=u, f_{2}(u, v, w)$ é função crescente de $v$ e $w$ e não depende de $u$, (com abuso da notação $f_{2}(u, v, w)=$ $\left.f_{2}(v, w)\right)$.

Definamos,

$$
\underline{S}:=\left(S_{1}, S_{2}\right)=\left(f_{1}\left(X_{1}, X_{2}, X_{3}\right), f_{2}\left(X_{1}, X_{2}, X_{3}\right)\right)=\left(X_{1}, f_{2}\left(X_{2}, X_{3}\right)\right)
$$

$\underline{S}$ è $P A$. 
2.5.6 Definição Seja $h: R^{2} \rightarrow R$ crescente que envia o par $(u, v)$ num numero real $z$. Suponhamos que. cada vez que fixamos a primeira coordenada, seja possível definir a aplicação inversa, assim, $h_{u}^{-1}: R \rightarrow R$ é a aplicação que para cada $z \in$ $R, h_{u}^{-1}(z)=v$, onde $h(u, v)=z$. Suponhamos que exista $\frac{\partial h}{\partial v}$.

\subsubsection{Proposição*}

Seja $h: R^{2} \rightarrow R$ crescente, satisfazendo 2.5.6. Consideremos o vetor aleatório $\left(X_{1}, X_{2}, X_{3}\right)$ tal que $\left(X_{1}, X_{3}\right)$ é $P A$; se

$$
f_{X_{1}, X_{3}}\left(x_{1}, h_{(\cdot)}^{-1}(z)\right)>_{T P_{2}(\cdot)} \frac{\partial h}{\partial v}\left(\cdot, h_{(\cdot)}^{-1}(z)\right)
$$

para todo $x_{1}$ na imagem de $X_{1}$ e (.) na imagem de $X_{2}, z \in R$. Então $X_{1}$ e $h\left(X_{2}, X_{3}\right)$ são $P A$.

Prova de 2.5.7:

sejam $U_{1}$ e $U_{2}$ variáveis aleatórias e $\underline{Z}$ vetor aleatório,

$$
\operatorname{Cov}\left\{U_{1}, U_{2}\right\}=E\left\{\operatorname{Cov}\left(U_{1}, U_{2} \mid \underline{Z}\right)\right\}+\operatorname{Cov}\left\{E\left(U_{1} \mid \underline{Z}\right), E\left(U_{2} \mid \underline{Z}\right)\right\} \quad\left(P_{1}\right) .
$$

lembremos que.

$$
\operatorname{Cov}\left\{\psi_{1}(X), \psi_{2}(X)\right\} \geq 0 \quad\left(P_{2}\right)
$$

onde $X$ representa qualquer $v \cdot a$. e $\psi_{i}, i=1,2$ são duas funções monótonas concordantes. 
Consideremos $\Gamma, \Delta: R^{2} \rightarrow R$ funções crescentes, binárias. Precisamos verificar que,

$$
\operatorname{Cov}\left\{\Gamma\left(X_{1}, h\left(X_{2}, X_{3}\right)\right), \Delta\left(X_{1}, h\left(X_{2}, X_{3}\right)\right)\right\} \geq 0
$$

e usando 2.5.1 (Barlow et al. (1981)), teríamos $X_{1}$ e $h\left(X_{2}, X_{3}\right) P A$.

Consideremos em $\left(P_{1}\right)$

$$
U_{1}:=\Gamma\left(X_{1}, h\left(X_{2}, X_{3}\right)\right), \quad U_{2}:=\Delta\left(X_{1}, h\left(X_{2}, X_{3}\right)\right), \quad \underline{Z}:=X_{2} .
$$

Notemos que, para cada $X_{2}=x_{2}$ fixo, as funções

$$
\Gamma\left(X_{1}, h\left(x_{2}, X_{3}\right)\right) \text { e } \Delta\left(X_{1}, h\left(x_{2}, X_{3}\right)\right)
$$

são funções crescentes e binárias de $X_{1}$ e $X_{3}$, pois a função $h$ é crescente e $\Gamma, \Delta$ são binárias crescentes. Como $\left(X_{1}, X_{3}\right)$ é $P A$, então,

$$
\operatorname{Cov}\left\{\Gamma\left(X_{1}, h\left(x_{2}, X_{3}\right)\right), \Delta\left(X_{1}, h\left(x_{2}, X_{3}\right)\right)\right\} \geq 0, \quad \forall x_{2} \in R \quad \text { (A). }
$$

Como por hipótese.

$$
f_{X_{1} \cdot X_{3}}\left(x_{1}, h_{(\cdot)}^{-1}(z)\right)>_{T P_{2}(\cdot)} \frac{\partial h}{\partial v}\left(\cdot, h_{(\cdot)}^{-1}(z)\right)
$$

sejam $x_{2}$ e $x_{2}^{\prime}$ na imagem de $X_{2}$ tais que $x_{2}<x_{2}^{\prime}$ temos,

$$
f_{X_{1}, X_{3}}\left(x_{1}, h_{x_{2}}^{-1}(u)\right) \frac{\partial h}{\partial x_{3}}\left(x_{2}^{\prime}, h_{x_{2}^{\prime}}^{-1}(u)\right) \leq f_{X_{1}, X_{3}}\left(x_{1}, h_{x_{2}^{\prime}}^{-1}(u)\right) \frac{\partial h}{\partial x_{3}}\left(x_{2}, h_{x_{2}}^{-1}(u)\right)
$$

por outra parte. para cada $x_{2}$ fixo,

$$
\begin{aligned}
E\left(\Gamma\left(X_{1}, h\left(x_{2}, X_{3}\right)\right)\right) & =\int_{-\infty}^{+\infty} \int_{-\infty}^{+\infty} \Gamma\left(x_{1}, h\left(x_{2}, x_{3}\right)\right) f_{X_{1}, X_{3}}\left(x_{1}, x_{3}\right) d x_{3} d x_{1} \\
& =\int_{-\infty}^{+\infty} \int_{-\infty}^{+\infty} \Gamma\left(x_{1}, u\right) f_{X_{1}, X_{3}}\left(x_{1}, h_{x_{2}}^{-1}(u)\right) \frac{d u}{\frac{\partial h\left(x_{2}, h_{x_{2}}^{-1}(u)\right)}{\partial x_{3}}} d x_{1}
\end{aligned}
$$


onde $u=h\left(x_{2}, x_{3}\right)$, portanto, $x_{3}=h_{x_{2}}^{-1}(u)$ e $d x_{3}=\frac{d u}{\frac{\partial h\left(x_{2}, h_{x_{2}}^{-1}(u)\right)}{\partial x_{3}}}$; pela desigualdade que notamos antes, é possível verificar

$$
E\left(\Gamma\left(X_{1}, h\left(x_{2}, X_{3}\right)\right)\right) \leq E\left(\Gamma\left(X_{1}, h\left(x_{2}^{\prime}, X_{3}\right)\right)\right) x_{2}<x_{2}^{\prime}
$$

Logo, $E\left(\Gamma\left(X_{1}, h\left(\cdot, X_{3}\right)\right)\right)$ é uma função crescente na imagem de $X_{2}$. De modo semelhante, $E\left(\Delta\left(X_{1}, h\left(\cdot, X_{3}\right)\right)\right)$ é uma função crescente na imagem de $X_{2}$. Finalmente, pela propriedade $\left(P_{2}\right)$, temos,

$$
\operatorname{Cov}\left\{E\left(\Gamma\left(X_{1}, h\left(X_{2}, X_{3}\right)\right) \mid X_{2}\right) . E\left(\Delta\left(X_{1}, h\left(X_{2}, X_{3}\right)\right) \mid X_{2}\right)\right\} \geq 0 \text { q.c. (B). }
$$

Agora, a partir de (A) e (B) conclui-se

$$
\operatorname{Cov}\left\{\Gamma\left(X_{1}, h\left(X_{2}, X_{3}\right)\right), \Delta\left(X_{1}, h\left(X_{2}, X_{3}\right)\right)\right\} \geq 0
$$

2.5.8 Exemplo : Seja o vetor $\left(X_{1}, X_{2}, X_{3}\right)$ com valores em $[a, b],[1,2],[2,3]$ respectivamente, por simplicidade assumimos $a>0$ e $b>0$. Onde cada um dos anteriores são intervalos da reta real. Suponhamos que a $f . d . p$. de $\left(X_{1}, X_{3}\right)$ é dada por $f_{X_{1}, X_{3}}(u, v)=c v e^{u v}$, se $u \in[a, b]$ e $v \in[2,3]$, e definida como 0 no caso contrário.

Logo, consideremos $h: R_{>0}^{2} \rightarrow R^{+}$, como $h\left(x_{2}, x_{3}\right):=x_{2}^{2}+x_{3}^{2}$. Notemos que

$$
\frac{\partial h}{\partial x_{3}}\left(x_{2}, x_{3}\right)=2 x_{3} ; \quad h_{x_{2}}^{-1}(z)=\left(z-x_{2}^{2}\right)^{\frac{1}{2}}, x_{2} \text { fixo. }
$$

Além disso, $f_{X_{1}, X_{3}}$ é $T P_{2}$ e

$$
\frac{f_{X_{1}, X_{3}}\left(x,\left(z-\left(x_{2}^{\prime}\right)^{2}\right)^{\frac{1}{2}}\right)}{\left(z-\left(x_{2}^{\prime}\right)^{2}\right)^{\frac{1}{2}}} \geq \frac{f_{X_{1}, X_{3}}\left(x,\left(z-x_{2}^{2}\right)^{\frac{1}{2}}\right)}{\left(z-x_{2}^{2}\right)^{\frac{1}{2}}}, \forall x_{1}, \quad x_{2}<x_{2}^{\prime}
$$

equivalentemente

$$
f_{X_{1}, X_{3}}\left(x, h_{(\cdot)}^{-1}(z)\right)>_{T P_{2}(\cdot)} \frac{\partial h}{\partial x_{3}}\left(\cdot, h_{(\cdot)}^{-1}(z)\right)
$$



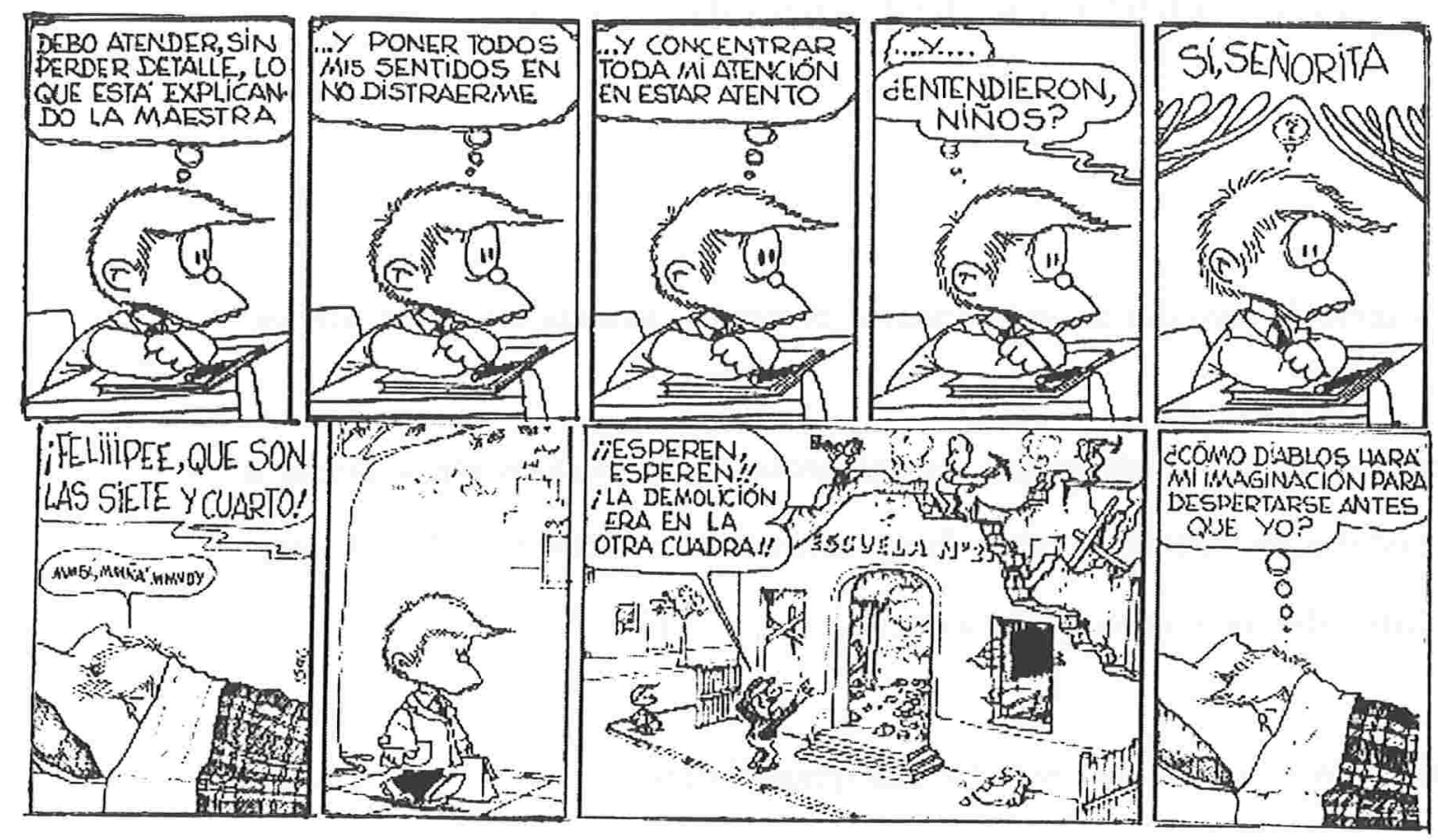

\section{Capítulo 3}

Medidas de Concordância e de Associação

- Seção 3.1: Medidas de Concordância

- Seção 3.2: Medida Adequada de Associação

- Seção 3.3: Existência do Acoplamento Associado

- Seção 3.4: Comportamento da medida $\tau$ de Kendall 
78

\subsection{Medidas de Concordância}

Objetivo

Estudo e origem de medidas de concordância bivariada, exemplos e propriedades.

3.1.1 Medidas de Concordância: previamente, apresentamos ideias gerais a respeito de medidas de associação entre duas variáveis aleatórias $X_{1}$ e $X_{2}$. Denotamos por $P$ a probabilidade vinculando $X_{1}$ e $X_{2}$.

As medidas da associação são baseadas nas quantidades

$$
P\left\{\left(X_{1}>x_{1,0}, X_{2}>x_{2,0}\right)\right\}, P\left\{\left(X_{1} \leq x_{1,0}, X_{2} \leq x_{2,0}\right)\right\}
$$

[Kruskal, W. (1958)].

As quantidades anteriores podem ser expressas em termos de

$$
P\left\{\left(X_{1}-x_{1,0}\right)\left(X_{2}-x_{2,0}\right) \geq 0\right\}
$$

A prévia quantidade é simplesmente a probabilidade de que o desvio de $X_{1}$ e $X_{2}$ a partir de $x_{1,0}$ e $x_{2,0}$, respectivamente, tenha o mesmo sinal; isto é, que $\left(X_{1}, X_{2}\right)$ esteja no primeiro e terceiro quadrantes determinados a partir do ponto $\left(x_{1,0} ; x_{2,0}\right) \in R^{2}$.

Por exemplo, poderíamos considerar $x_{i, 0}:=\operatorname{Med}\left(X_{i}\right)$, mediana de $X_{i}, i=1,2$. 
Logo definimos

$$
\sigma_{c}:=P\left\{\left(X_{1}-\operatorname{Med}\left(X_{1}\right)\right)\left(X_{2}-\operatorname{Med}\left(X_{2}\right)\right) \geq 0\right\}
$$

como sendo a probabilidade de que os desvios de $X_{1}$ e $X_{2}$ a partir de suas respectivas medianas tenham o mesmo sinal. A probabilidade de concordância, de sinal, $\sigma_{c}$ toma valores entre 0 e 1 inclusive. Ela é 1 se, e somente se, $X_{1}-\operatorname{Med}\left(X_{1}\right)$ e $X_{2}-\operatorname{Med}\left(X_{2}\right)$ são ambas não negativas ou ambas negativas com probabilidade 1. As duas condições são mutuamente excludentes. E ela é 0 se, e somente se, $X_{1}-\operatorname{Med}\left(X_{1}\right)$ e $X_{2}-\operatorname{Med}\left(X_{2}\right)$ têm diferente sinal com probabilidade 1 . Se $X_{1}$ e $X_{2}$ são independentes, $\sigma_{c}=\frac{1}{2}$, mas a recíproca não é válida, em geral. Consideremos a seguir,

$$
\sigma_{d}:=P\left\{\left(X_{1}-\operatorname{Med}\left(X_{1}\right)\right)\left(X_{2}-\operatorname{Med}\left(X_{2}\right)\right)<0\right\},
$$

ou a probabilidade de que o vetor $\left(X_{1}, X_{2}\right)$ esteja no segundo e quarto quadrantes do sistema de coordenadas de origem em $\left(\operatorname{Med}\left(X_{1}\right), \operatorname{Med}\left(X_{2}\right)\right)$. Equivalentemente $\sigma_{d}$ é a probabilidade de que $X_{1}-\operatorname{Med}\left(X_{1}\right)$ e $X_{2}-\operatorname{Med}\left(X_{2}\right)$ tenham sinais diferentes. Evidentemente $\sigma_{c}+\sigma_{d}=1$.

Com essas duas probabilidades, é definida uma quantidade natural.

$$
\tau_{X_{1}, X_{2}}^{\operatorname{Med}\left(X_{1}\right), \operatorname{Med}\left(X_{2}\right)}:=\sigma_{c}-\sigma_{d}=2 \sigma_{c}-1 .
$$

Notemos que $\tau_{X_{1}, X_{2}}^{\operatorname{Med}\left(X_{1}\right), \operatorname{Med}\left(X_{2}\right)}=1$ se, e somente se, $X_{1}$ e $X_{2}$ desviam na mesma direção a partir das respectivas medianas. $\tau_{X_{1}, X_{2}}^{\operatorname{Med}\left(X_{1}\right), \operatorname{Med}\left(X_{2}\right)}=-1 \mathrm{se}$, e somente se, $X_{1}$ e $X_{2}$ desviam em direções opostas a partir das respectivas medianas. Se $X_{1}$ e $X_{2}$ são independentes, então, $\tau_{X_{1}, X_{2}}^{\operatorname{Med}\left(X_{1}\right), \operatorname{Med}\left(X_{2}\right)}=0$; mas a recíproca não é sempre válida. Assim, $\tau_{X_{1}, X_{2}}^{\operatorname{Med}\left(X_{1}\right), \operatorname{Med}\left(X_{2}\right)}$ define uma medida de concordância cuja imagem é $[-1,1]$, adquirindo o valor 0 no caso de independência. 
O significado da medida anterior talvez possa ser esclarecido com o seguinte exemplo:

Suponhamos que é de nosso interesse estudar a associação entre dois testes de inteligência, quando se submete uma mesma população a ambos os testes. A pontuação nos dois testes corresponde às variáveis aleatórias $X_{1}$ e $X_{2}$ respectivamente. $\tau_{X_{1}, X_{2}}^{\operatorname{Med}\left(X_{1}\right), \operatorname{Med}\left(X_{2}\right)}$ é uma mensuração de que as pontuações tiradas pela população como resposta aos testes de inteligência tenham um desvio

$$
\frac{\tau_{X_{1}, X_{2}}^{\operatorname{Med}\left(X_{1}\right), \operatorname{Med}\left(X_{2}\right)}+1}{2},
$$

na mesma direção, com relação às medianas.

Se agora considerássemos todas as probabilidades possíveis, calculadas para valores arbitrários $\left(x_{1,0} ; x_{2,0}\right)$, isto é $P\left(\left(X_{1}-x_{1,0}\right)\left(X_{2}-x_{2,0}\right) \geq 0\right) \forall\left(x_{1,0} ; x_{2,0}\right)$, podemos fabricar outra quantidade, globalmente mais representativa.

$$
\pi_{c}:=P\left(\left(X_{1}-X_{1}^{\prime}\right)\left(X_{2}-X_{2}^{\prime}\right) \geq 0\right)
$$

e, de forma equivalente,

$$
\pi_{d}:=P\left(\left(X_{1}-X_{1}^{\prime}\right)\left(X_{2}-X_{2}^{\prime}\right)<0\right)
$$

onde $\left(X_{1}, X_{2}\right)$ e $\left(X_{1}^{\prime}, X_{2}^{\prime}\right)$ são tomados como dois vetores aleatórios independentes com a mesma distribuição. A saber, $\pi_{c}$ é a probabilidade de que duas observações hipotéticas bivariadas, sobre a distribuição bivariada de interesse. sejam concordantes. $\pi_{d}$ tem um significado semelhante, mas para discordância. 
É preciso notar que $\pi_{c}$ para as variáveis $\left(X_{1}, X_{2}\right)$ é igual a calcular $\sigma_{c}$ para $\left(X_{1}-\right.$ $\left.X_{1}^{\prime}, X_{2}-X_{2}^{\prime}\right)$, onde, naturalmente, $\left(X_{1}, X_{2}\right)$ e $\left(X_{1}^{\prime}, X_{2}^{\prime}\right)$ são independentes com a mesma distribuição, e $\operatorname{Med}\left(X_{1}-X_{1}^{\prime}\right)=\operatorname{Med}\left(X_{2}-X_{2}^{\prime}\right)=0$.

Assim, a medida de associação baseada nas quantidades $\pi_{c}$ e $\pi_{d}$ é a medida $\tau$ de Kendall. A mesma é definida para o par de variáveis aleatórias $X_{1}, X_{2}$, como segue,

$$
\tau_{X_{1}, X_{2}}:=\pi_{c}-\pi_{d}
$$

Notemos que $\tau_{X_{1}, X_{2}}=\tau_{V_{1}, V_{2}}^{\operatorname{Med}\left(V_{1}\right), \operatorname{Med}\left(V_{2}\right)}$, onde $V_{1}:=X_{1}-X_{1}^{\prime}$ e $V_{2}:=X_{2}-X_{2}^{\prime}$, descritos anteriormente, ou também podemos interpretar o $\tau_{X_{1}, X_{2}}$ como o coeficiente de correlação entre o $\operatorname{sinal}\left(X_{1}-X_{1}^{\prime}\right)$ e o $\operatorname{sinal}\left(X_{2}-X_{2}^{\prime}\right)$. É por isso que muitas vezes o $\tau$ de Kendall é chamado "correlação sinal diferença". Hoeffding chama-o de " covariância sinal diferença" (pois $\left.\operatorname{Var}\left[\operatorname{sinal}\left(X_{1}-X_{1}^{\prime}\right)\right]=\operatorname{Var}\left[\operatorname{sinal}\left(X_{2}-X_{2}^{\prime}\right)\right]\right)$.

Interpretemos $\tau$ como a esperança de ganhar "um dólar". Suponhamos que as observações $\left(X_{1}, X_{2}\right)$ e $\left(X_{1}^{\prime}, X_{2}^{\prime}\right)$ estão disponíveis, mas primeiro são revelados os valores de $X_{1}$ e $X_{2}$, por algum recurso mecânico. Suponhamos que participamos de um jogo onde predizemos $X_{2}<X_{2}^{\prime}$ quando $X_{1}<X_{1}^{\prime}$ e $X_{2}>X_{2}^{\prime}$ quando $X_{1}>X_{1}^{\prime}$. Se nossa predição for correta, ganhamos um dólar, caso contrário, perdemos um dólar. Uma vez comunicada a predição. o recurso mecânico revela $X_{2}$ e $X_{2}^{\prime}$, concluindo-se o jogo. Assim, a esperança de ganhar um dólar é $\tau_{X_{1}, X_{2}}$.

No exemplo dos testes de inteligência, suponhamos que estamos interessados em conhecer a associação entre ambos os testes de inteligência, para uma população específica. Tomemos aleatoriamente dois indivíduos da população de interesse e 
comparemos os resultados dos testes para ambos os indivíduos. $\pi_{c}$ é a probabilidade de que o mais inteligente, de acordo com um dos testes, seja também o mais inteligente de acordo com o outro teste. $\pi_{d}$ é a probabilidade de que essas ordens difiram. E $\tau$ é justamente a diferença entre essas probabilidades.

Outras propriedades [Kruskal, W.(1958); Genest, Ch. e MacKay, J.(1986); Joe, H.(1997)]

ค $\tau_{X_{1}, X_{2}}=\tau_{X_{1}-X_{1}^{\prime}, X_{2}-X_{2}^{\prime}}^{\operatorname{Med}\left(X_{1}-X_{2}^{\prime}\right) \operatorname{Med}\left(X_{2}-X_{2}^{\prime}\right)}, \operatorname{Med}\left(X_{i}-X_{i}^{\prime}\right)=0, \quad i=1,2$.

A $\tau_{X_{1}, X_{2}}=\rho_{\text {sinal }\left(X_{1}-X_{1}^{\prime}\right), \operatorname{sinal}\left(X_{2}-X_{2}^{\prime}\right)}$ onde $\left(X_{1}, X_{2}\right)$ e $\left(X_{1}^{\prime}, X_{2}^{\prime}\right)$ são independentes e ambos possuem a mesma f.d.a. $F_{X_{1}, X_{2}}$,

$$
\operatorname{var}\left[\operatorname{sinal}\left(X_{1}-X_{1}^{\prime}\right)\right]=\operatorname{var}\left[\operatorname{sinal}\left(X_{2}-X_{2}^{\prime}\right)\right]=1
$$

A A relação existente entre a medida $\tau_{X_{1}, X_{2}}$ e o coeficiente de correlação $\rho_{U_{1}, U_{2}}$ onde $U_{i}:=F_{X_{i}}\left(X_{i}\right)$ e $F_{X_{i}}$ é a $f . d . a$. da variável $X_{i}$, contínua, $i=1$, 2. é a seguinte

$$
-1 \leq 3 \tau_{X_{1}, X_{2}}-2 \rho_{U_{1}, U_{2}} \leq 1
$$

$-1 \leq \tau_{X_{1}, X_{2}} \leq 1$. 


\subsubsection{Proposição}

Sejam $\left(X_{1}^{\prime}, X_{2}^{\prime}\right),\left(X_{1}^{\prime \prime}, X_{2}^{\prime \prime}\right)$ dois vetores independentes, realizações da mesma distribuição, de marginais acumuladas $F_{X_{1}}$ e $F_{X_{2}}$, contínuas. Então,

(i)

$$
\tau_{X_{1}, X_{2}}=2 P\left(\left(X_{1}^{\prime}-X_{1}^{\prime \prime}\right)\left(X_{2}^{\prime}-X_{2}^{\prime \prime}\right) \geq 0\right)-1
$$

(ii) Sejam $f_{1}$ e $f_{2}$ duas aplicações, não decrescentes; $T_{i}:=f_{i}\left(X_{i}\right), i=1,2$;

$$
\tau_{T_{1}, T_{2}}=\tau_{X_{1}, X_{2}}
$$

(iii) $\tau_{X_{1}, X_{2}}=-\tau_{X_{1},-X_{2}}$.

(iv) Sejam $g_{1}$ e $g_{2}$ duas aplicações monótonas discordantes (i.e., uma delas não decrescente e a outra não crescente). $S_{i}:=g_{i}\left(X_{i}\right), i=1,2$;

$$
\tau_{X_{1}, X_{2}}=-\tau_{S_{1}, S_{2}}
$$

Prova de 3.1.2:

(i) : É claro que

$$
P\left(\left(X_{1}^{\prime}-X_{1}^{\prime \prime}\right)\left(X_{2}^{\prime}-X_{2}^{\prime \prime}\right) \geq 0\right)+P\left(\left(X_{1}^{\prime}-X_{1}^{\prime \prime}\right)\left(X_{2}^{\prime}-X_{2}^{\prime \prime}\right)<0\right)=1,
$$

onde $\left(X_{1}^{\prime}, X_{2}^{\prime}\right)$ e $\left(X_{1}^{\prime \prime}, X_{2}^{\prime \prime}\right)$ são dois pares de vetores aleatórios independentes, ambos com f.d.a. $F_{X_{1}, X_{2}}$. Logo,

$$
\tau_{X_{1}, X_{2}}=2 P\left(\left(X_{1}^{\prime}-X_{1}^{\prime \prime}\right)\left(X_{2}^{\prime}-X_{2}^{\prime \prime}\right) \geq 0\right)-1
$$


Notemos que em geral, ante a ausência da hipótese das marginais contínuas, a medida $\tau$ terá a seguinte expressão

$$
\begin{aligned}
\tau_{X_{1}, X_{2}}= & 2 P\left(X_{1}^{\prime} \leq X_{1}^{\prime \prime}, X_{2}^{\prime} \leq X_{2}^{\prime \prime}\right)-P\left(X_{1}^{\prime} \leq X_{1}^{\prime \prime}\right) \\
& -P\left(X_{2}^{\prime} \leq X_{2}^{\prime \prime}\right)+1+P\left(X_{1}^{\prime}=X_{1}^{\prime \prime}, X_{2}^{\prime}>X_{2}^{\prime \prime}\right) \\
& +P\left(X_{1}^{\prime}>X_{1}^{\prime \prime}, X_{2}^{\prime}=X_{2}^{\prime \prime}\right) \\
& -P\left(X_{1}^{\prime}>X_{1}^{\prime \prime}, X_{2}^{\prime}<X_{2}^{\prime \prime}\right)-P\left(X_{1}^{\prime}<X_{1}^{\prime \prime}, X_{2}^{\prime}>X_{2}^{\prime \prime}\right)
\end{aligned}
$$

A expressão prévia serve para a implementação do cálculo computacional da medida $\tau$, no caso discreto.

(ii): Definamos

$$
\eta_{1,2}:=P\left\{\left(X_{1}^{\prime}-X_{1}^{\prime \prime}\right)\left(X_{2}^{\prime}-X_{2}^{\prime \prime}\right) \geq 0\right\}
$$

como as marginais $F_{X_{i}}, i=1,2$ são contínuas,

$$
\eta_{1,2}=P\left(X_{1}^{\prime} \leq X_{1}^{\prime \prime}, X_{2}^{\prime} \leq X_{2}^{\prime \prime}\right)+P\left(X_{1}^{\prime}>X_{1}^{\prime \prime}, X_{2}^{\prime}>X_{2}^{\prime \prime}\right)
$$

Notemos que, como $f_{1}$ e $f_{2}$ são não decrescentes temos para $i=1,2$,

$$
\begin{aligned}
& \left\{X_{i}^{\prime} \leq X_{i}^{\prime \prime}\right\} \subseteq\left\{f_{i}\left(X_{i}^{\prime}\right) \leq f_{i}\left(X_{i}^{\prime \prime}\right)\right\}, \quad\left\{f_{i}\left(X_{i}^{\prime}\right)<f_{i}\left(X_{i}^{\prime \prime}\right)\right\} \subseteq\left\{X_{i}^{\prime}<X_{i}^{\prime \prime}\right\} \\
& \left\{X_{i}^{\prime} \geq X_{i}^{\prime \prime}\right\} \subseteq\left\{f_{i}\left(X_{i}^{\prime}\right) \geq f_{i}\left(X_{i}^{\prime \prime}\right)\right\}, \quad\left\{f_{i}\left(X_{i}^{\prime}\right)>f_{i}\left(X_{i}^{\prime \prime}\right)\right\} \subseteq\left\{X_{i}^{\prime}>X_{i}^{\prime \prime}\right\} .
\end{aligned}
$$

Por outro lado pela continuidade das marginais $F_{X_{1}}$ e $F_{X_{2}}$, temos

$$
\begin{aligned}
P\left(X_{1}^{\prime} \leq X_{1}^{\prime \prime}, X_{2}^{\prime} \leq X_{2}^{\prime \prime}\right) & \leq P\left(f_{1}\left(X_{1}^{\prime}\right) \leq f_{1}\left(X_{1}^{\prime \prime}\right), f_{2}\left(X_{2}^{\prime}\right) \leq f_{2}\left(X_{2}^{\prime \prime}\right)\right) \\
& =P\left(f_{1}\left(X_{1}^{\prime}\right)<f_{1}\left(X_{1}^{\prime \prime}\right), f_{2}\left(X_{2}^{\prime}\right)<f_{2}\left(X_{2}^{\prime \prime}\right)\right) \\
& \leq P\left(X_{1}^{\prime}<X_{1}^{\prime \prime}, X_{2}^{\prime}<X_{2}^{\prime \prime}\right),
\end{aligned}
$$


assim,

$$
P\left(X_{1}^{\prime} \leq X_{1}^{\prime \prime}, X_{2}^{\prime} \leq X_{2}^{\prime \prime}\right)=P\left(f_{1}\left(X_{1}^{\prime}\right) \leq f_{1}\left(X_{1}^{\prime \prime}\right), f_{2}\left(X_{2}^{\prime}\right) \leq f_{2}\left(X_{2}^{\prime \prime}\right)\right),
$$

e similarmente

$$
P\left(X_{1}^{\prime}>X_{1}^{\prime \prime}, X_{2}^{\prime}>X_{2}^{\prime \prime}\right)=P\left(f_{1}\left(X_{1}^{\prime}\right)>f_{1}\left(X_{1}^{\prime \prime}\right), f_{2}\left(X_{2}^{\prime}\right)>f_{2}\left(X_{2}^{\prime \prime}\right)\right)
$$

segue que

$$
\tau_{X_{1}, X_{2}}=\tau_{T_{1}, T_{2}} .
$$

(iii) : Notemos que

$$
\left\{\left(X_{1}^{\prime}-X_{1}^{\prime \prime}\right)\left(X_{2}^{\prime}-X_{2}^{\prime \prime}\right) \geq 0\right\}=\left\{\left(X_{1}^{\prime}-x_{1}^{\prime \prime}\right)\left(-X_{2}^{\prime}+X_{2}^{\prime \prime}\right) \leq 0\right\},
$$

como $F_{X_{1}}$ e $F_{X_{2}}$ são contínuas, temos

$$
P\left\{\left(X_{1}^{\prime}-x_{1}^{\prime \prime}\right)\left(-X_{2}^{\prime}+X_{2}^{\prime \prime}\right) \leq 0\right\}=P\left\{\left(X_{1}^{\prime}-x_{1}^{\prime \prime}\right)\left(-X_{2}^{\prime}+X_{2}^{\prime \prime}\right)<0\right\},
$$

assim

$$
\tau_{X_{1}, X_{2}}=-\tau_{X_{1},-X_{2}} .
$$

(iv) : Resulta como conseqüência de (ii) e (iii). 
86

\subsection{Medida Adequada de Associação}

\section{Objetivos}

- Considerando a medida de concordância $\tau_{X_{1}, X_{2}}$ de Kendall para o par $\left(X_{1}, X_{2}\right)$, dar sua expressão no caso de densidades marginais contínuas e no caso discreto.

- Determinar "quando" a medida de concordância $\tau$ de Kendall constitui uma medida de associação, tanto positiva como negativa.

- Fornecer exemplos das diversas situações.

Nesta seção, procuramos uma medida que interprete a condição $M T P_{2}\left(M R R_{2}\right)$, no caso bivariado, pois estamos interessados em variáveis aleatórias $P A(N A)$, no caso em que as densidades bivariadas são $T P_{2}\left(R R_{2}\right)$; ou seja, densidades conjuntas $T P_{2}\left(R R_{2}\right)$ em pares.

A seguir, apresentamos alguns tópicos que permitiram estabelecer as propriedades da medida $\tau$ de Kendall, na sua relação com o conceito de associação (positiva ou negativa) e sua conexão com as densidades de marginais contínuas.

O seguinte teorema será de extrema utilidade para o desenvolvimento dinâmico da seção. Encontra-se provado detalhadamente em [Mikusinski et al. (1991)]. 
3.2.1 Teorema [Fréchet (1951), Mikusinski (1991), Joe (1997) pp.58]

Seja $\left(X_{1}, \cdots, X_{n}\right)$ um vetor aleatório com função de distribuição acumulada conjunta $F_{X_{1}, \cdots, X_{n}}$ de marginais univariadas acumuladas, $\left\{F_{X_{i}}\right\}_{i=1}^{n}$. Então, $\max \left\{0, \sum_{i=1}^{n} F_{X_{i}}\left(x_{i}\right)-(n-1)\right\} \leq F_{X_{1}, \cdots, X_{n}}\left(x_{1}, \cdots, x_{n}\right) \leq \min \left\{F_{X_{1}}\left(x_{1}\right), \cdots, F_{X_{n}}\left(x_{n}\right)\right\}$, $\forall n$-upla $\left(x_{1}, \cdots, x_{n}\right)$ na imagem do vetor aleatório $\left(X_{1}, \cdots, X_{n}\right)$.

Notemos que, quando as marginais univariadas são uniformes em $(0,1) 3.2 .1$ fornece limitantes para todos os acoplamentos possíveis. Por exemplo, se $n=2$

$$
\max \left\{0, x_{1}+x_{2}-1\right\} \leq C_{X_{1}, X_{2}}\left(x_{1}, x_{2}\right) \leq \min \left\{x_{1}, x_{2}\right\}, \quad \forall x_{i} \in[0,1], i=1,2 .
$$

Vide [Nelsen (1995) e Figura 3.2.2]

Comentário: Notemos que a f.d.a. dada pelo $\min \left\{x_{1}, x_{2}\right\}$ em $[0,1]^{2}$ é uma função $T P_{2}$ entanto que a f.d.a. dada por $\max \left\{0, x_{1}+x_{2}-1\right\}$ em $[0,1]^{2}$ é $R R_{2}$, e entre essas duas funções encontram-se todas as distribuições em $R^{2}$, cujas marginais são $U(0,1)$. Que informação pode ser tirada respeito das variáveis aleatórias que possuem uma u outra distribuição extrema?. Que pode ser dito respeito da medida de associação $\tau$ de Kendall?. Todas essas questões serão respondidas no Capítulo 4.

O ponto 3.2.3 apresentado a seguir, terá uma seção (3 deste Capítulo) dedicada ao desenvolvimento da prova. 
3.2.3 Teorema [Sklar (1959)]

Seja $\left(X_{1}, \cdots, X_{n}\right)$ um vetor aleatório com função de distribuição acumulada conjunta $F_{X_{1}, \cdots, X_{n}}$ de marginais univariadas acumuladas $\left\{F_{X_{i}}\right\}_{i=1}^{n}$. Então, existe algum acoplamento $C_{X_{1}, \cdots, X_{n}}$ (f.d.a. de marginais univariadas $U(0,1)$ ), associado à f.d.a. $F_{X_{1}, \cdots, X_{n}}$.

As duas proposições seguintes são conseqüencias das definições envolvidas nelas.

\subsubsection{Proposição*}

Seja o vetor aleatório $\left(X_{1}, X_{2}\right)$ com f.d.a. conjunta $F_{X_{1}, X_{2}}$ e acoplamento associado $C_{X_{1}, X_{2}}$. Então, se $C_{X_{1}, X_{2}}$ é $T P_{2}\left(R R_{2}\right)$ em intervalos, $F_{X_{1}, X_{2}}$ é $T P_{2}\left(R R_{2}\right)$ em intervalos.

\subsubsection{Proposição [Joe (1997)]}

Seja o vetor aleatório $\left(X_{1}, X_{2}\right)$ com função de densidade da probabilidade conjunta $f_{X_{1}, X_{2}} T P_{2}\left(R R_{2}\right)$. Então, a função acumulada conjunta $F_{X_{1}, X_{2}}$ é $T P_{2}\left(R R_{2}\right)$, conseqüentemente, $F_{X_{1}, X_{2}}$ é $T P_{2}\left(R R_{2}\right)$ em intervalos.

A seguir, apresentamos 3.2 .6 e 3.2 .8 , que serão provados oportunamente. 
figura 3.2.2: Acoplamentos Extremos e Acoplamento de Independência

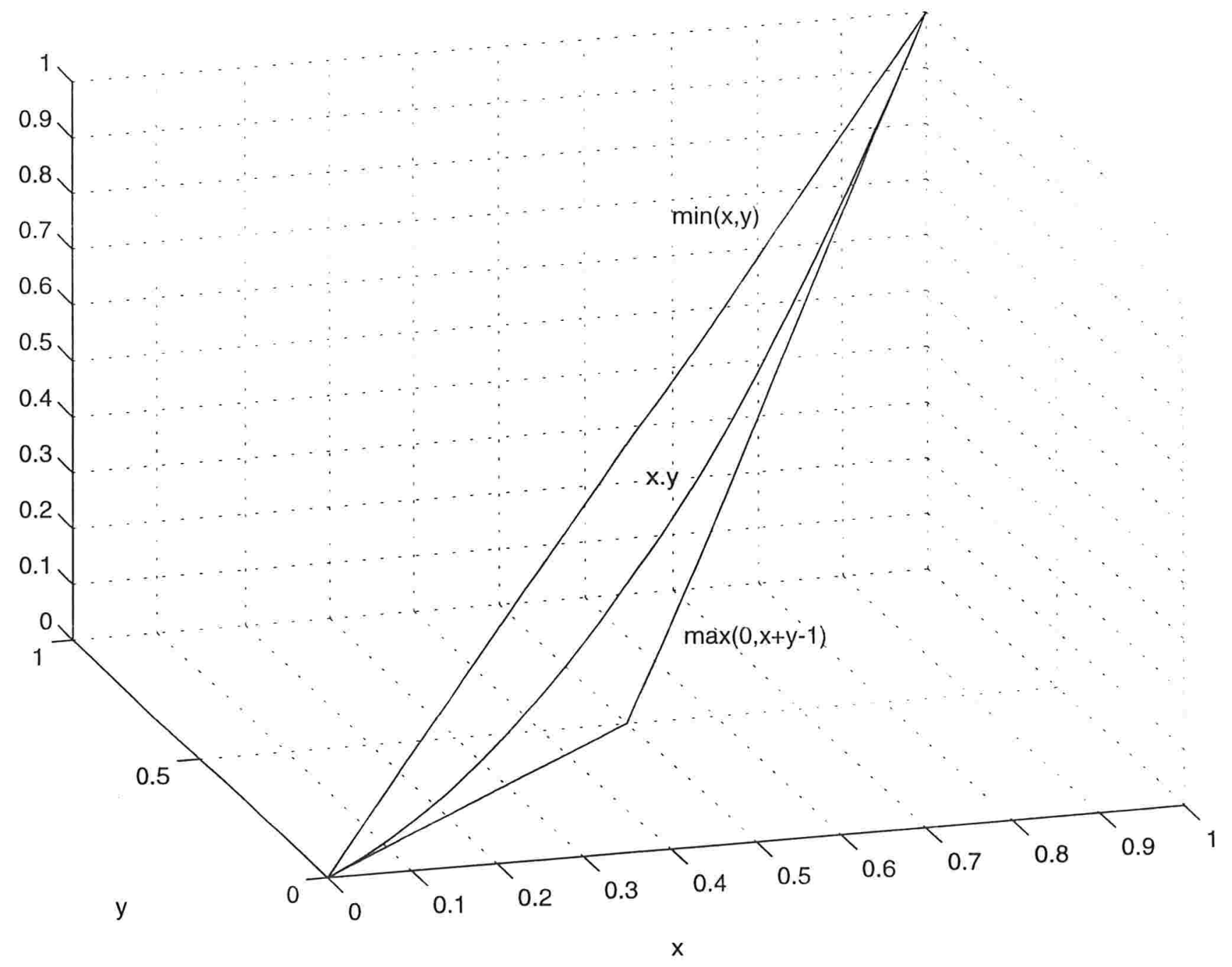


3.2.6 Proposição Seja $\left(X_{1}, X_{2}\right)$ um vetor aleatório, $F_{X_{1}, X_{2}}$ sua $f . d . a$. conjunta.

(i) Se as marginais acumuladas univariadas $F_{X_{1}}$ e $F_{X_{2}}$ são contínuas, então,

$$
\tau_{X_{1}, X_{2}}=4 \int F_{X_{1}, X_{2}}\left(x_{1}, x_{2}\right) d F_{X_{1}, X_{2}}\left(x_{1}, x_{2}\right)-1
$$

(ii) Se $F_{X_{1}, X_{2}}$ for absolutamente contínua, com f.d.p. conjunta $f_{X_{1}, X_{2}}$ e acoplamento associado $C_{X_{1}, X_{2}}$. Então,

$$
\tau_{X_{1}, X_{2}}=4 \int_{[0,1]^{2}} C_{X_{1}, X_{2}}\left(u_{1}, u_{2}\right) d C_{X_{1}, X_{2}}\left(u_{1}, u_{2}\right)-1 .
$$

Onde, em ambos os casos, $\tau_{X_{1}, X_{2}}$ denota a medida $\tau$ de Kendall para as variáveis $X_{1}$ e $X_{2}$.

\subsubsection{Definição}

Dizemos que as variáveis aleatórias $X_{1}$ e $X_{2}$ são $P A\left(T P_{2}\right)$ se a f.d.p. conjunta for $T P_{2}$.

Dizemos que as variáveis aleatórias $X_{1}$ e $X_{2}$ são $N A\left(R R_{2}\right)$ se a $f . d . p$. conjunta for $R R_{2}$. 
3.2.8 Teorema [Nelsen (1992)]

(i) Sejam $X_{1}, X_{2}$ variáveis aleatórias com f.d.a. conjunta $F_{X_{1}, X_{2}}$, absolutamente contínua e f.d.p. conjunta $f_{X_{1}, X_{2}}$.

Seja $T_{X_{1}, X_{2}}$ a média das medidas "locais" da condição $T P_{2}\left(R R_{2}\right)$, definida por

$$
\begin{aligned}
T_{X_{1}, X_{2}} & :=\int_{-\infty}^{+\infty} \int_{-\infty}^{+\infty} \int_{-\infty}^{x_{2}^{\prime}} \int_{-\infty}^{x_{1}^{\prime}}\left[f_{X_{1}, X_{2}}\left(x_{1}^{\prime}, x_{2}^{\prime}\right) f_{X_{1}, X_{2}}\left(x_{1}, x_{2}\right)\right. \\
& \left.-f_{X_{1}, X_{2}}\left(x_{1}, x_{2}^{\prime}\right) f_{X_{1}, X_{2}}\left(x_{1}^{\prime}, x_{2}\right)\right] d x_{1} d x_{2} d x_{1}^{\prime} d x_{2}^{\prime}
\end{aligned}
$$

(implícitamente $-\infty<x_{1}<x_{1}^{\prime}<\infty$ e $-\infty<x_{2}<x_{2}^{\prime}<\infty$ ). Então,

$$
T_{X_{1}, X_{2}}=\frac{\tau_{X_{1}, X_{2}}}{2} .
$$

Nessas condições, se a f.d.p., $f_{X_{1}, X_{2}}$ é $T P_{2}\left(R R_{2}\right)$, então $\tau_{X_{1}, X_{2}}$ é uma "medida de associação" $T P_{2}\left(R R_{2}\right)$, pois $\tau_{X_{1}, X_{2}}$ é uma média ponderada das medidas "locais" da condição $T P_{2}\left(R R_{2}\right)$.

(ii) Se a f.d.a. $F_{X_{1}, X_{2}}$ é $T P_{2}\left(R R_{2}\right)$ em intervalos, $C_{X_{1}, X_{2}}$ é o acoplamento associado à f.d.a. Sejam $\left\{u_{p}\right\}_{p=0}^{n}, 0=u_{0}<u_{1}<\cdots<u_{n}$ e $\left\{v_{q}\right\}_{q=0}^{m}, 0=v_{0}<v_{1}<$ $\cdots<v_{m}$ partições de $I=[0,1]$, definindo $J_{p}=\left[u_{p-1}, u_{p}\right]$ e $K_{q}=\left[v_{q-1}, v_{q}\right]$ temos uma partição de $I^{2}, P$, em $m n$ retângulos $J_{p} \times K_{q}$. Denotamos por $\|P\|$ a norma de $P$ - norma do ínfimo.

Então, se $1 \leq r<p \leq n$ e $1 \leq s<q \leq m$,

$$
C_{X_{1}, X_{2}}\left(J_{p}, K_{q}\right) C_{X_{1}, X_{2}}\left(J_{r}, K_{s}\right)-C_{X_{1}, X_{2}}\left(J_{p}, K_{s}\right) C_{X_{1}, X_{2}}\left(J_{r}, K_{q}\right)
$$

representa uma medida "local" da condição $T P_{2}\left(R R_{2}\right)$ em intervalos. Onde

$$
C\left(\left[u_{1}, u_{2}\right],\left[v_{1}, v_{2}\right]\right):=C\left(u_{2}, v_{2}\right)-C\left(u_{1}, v_{2}\right)-C\left(u_{2}, v_{1}\right)+C\left(u_{1}, v_{1}\right)
$$


Se definimos

$$
\begin{gathered}
T_{X_{1}, X_{2}}:=\lim _{\|P\| \rightarrow 0} \sum_{p=2}^{n} \sum_{q=2}^{m} \sum_{r=1}^{p-1} \sum_{s=1}^{q-1}\left[C_{X_{1}, X_{2}}\left(J_{p}, K_{q}\right) C_{X_{1}, X_{2}}\left(J_{r}, K_{s}\right)\right. \\
\left.-C_{X_{1}, X_{2}}\left(J_{p}, K_{s}\right) C_{X_{1}, X_{2}}\left(J_{r}, K_{q}\right)\right],
\end{gathered}
$$

$T_{X_{1}, X_{2}}$ existe $\mathrm{e}$

$$
T_{X_{1}, X_{2}}=\frac{\tau_{X_{1}, X_{2}}}{2}
$$

sempre que $F_{X_{1}, X_{2}}$ seja absolutamente contínua (para ter

$$
\left.\tau_{X_{1}, X_{2}}=4 \int_{I^{2}} C_{X_{1}, X_{2}} d C_{X_{1}, X_{2}}-1 .\right)
$$

\subsubsection{Observações}

(i) Nem sempre a medida de concordância $\tau$ representa uma medida de associação $T P_{2}\left(R R_{2}\right)$.

(ii) Se as marginais acumuladas são contínuas e a densidade (acumulada ou de probabilidade) for da classe $T P_{2}\left(R R_{2}\right)$, então, escolhemos a $\tau$ como medida de associação.

(iii) Se as marginais acumuladas não são contínuas, mas a densidade (acumulada ou de probabilidade), for da classe $T P_{2}\left(R R_{2}\right)$, então, a medida de associação, no sentido apresentado é a quantidade $T$, definida em 3.2.8.

Em seguida, desenvolvemos cada um dos tópicos, anteriormente mencionados. 


\subsection{Existência do Acoplamento Associado}

3.3.1 De nição Seja $F_{X}: R \rightarrow[0,1]$ a função de distribuição acumulada contínua, da variável aleatória $X$. Definamos, $\forall u \in(0,1) F_{X}^{q i}$ (quase-inversa)

$$
\begin{aligned}
F_{X}^{q i}: & (0,1) \rightarrow R \\
& u \rightarrow \inf \left\{x \in R: F_{X}(x) \geq u\right\}
\end{aligned}
$$

Notemos que a função anterior está bem definida $\forall u \in(0,1)$.

Tomemos qualquer valor $u \in(0,1)$. Como $\lim _{x \rightarrow+\infty} F_{X}(x)=1$ e $F_{X}$ é contínua não decrescente, existe $z_{1} \in R$ tal que $F_{X}\left(z_{1}\right)>u$. Assim, $\left\{x \in R: F_{X}(x) \geq u\right\}$ é não vazio. No entanto. como $\lim _{x \rightarrow-\infty} F_{X}(x)=0$, existe $z_{0} \in R$ tal que $F_{X}\left(z_{0}\right)<u$, logo, $z_{0}$ é limitante inferior de $\left\{x \in R: F_{X}(x) \geq u\right\}$.

Mais geralmente, o conjunto $L:=\left\{x: F_{X}(x)<u\right\}$ é um conjunto de limitantes inferiores de $\left\{x: F_{X}(x) \geq u\right\}$. Suponhamos, pois, que exista algum $z \in L$ e algum elemento $x \in\left\{x: F_{X}(x) \geq u\right\}$, tal que $z>x$, então, $F_{X}(z) \geq F_{X}(x) \geq u$. Isso levaria a concluir que $z$ não pertence ao $L$ : absurdo.

Finalmente, pelo axioma da completitude de $R,\left\{x \in R: F_{X}(x) \geq u\right\}$ admite ínfimo, qualquer que seja $u \in(0,1)$. 
Por outro lado, $\left\{x: F_{X}(x) \geq u\right\}=F_{X}^{-1}([u, 1])$, i.e. a preimagem de um intervalo fechado na reta, por uma função contínua $F_{X}$. Logo, o ínfimo existente é um mínimo do conjunto.

Cabe destacar que, se $F_{X}$ é estritamente crescente, a função acima definida $F_{X}^{q i}$ coincide com a inversa usual.

\subsubsection{Lema}

Sejam $F_{X}: R \rightarrow[0,1]$ uma função de distribuição acumulada contínua e $F_{X}^{q i}$ a quase-inversa da $F_{X}$. Então, $\forall u \in(0,1)$ e $\forall t \in R$

$$
F_{X}^{q i}(u) \leq t \Leftrightarrow u \leq F_{X}(t)
$$

Prova de 3.3.2:

Consideremos uma seqüência não crescente $\left\{x_{n}: n \geq 1\right\} \subseteq\left\{x \in R: F_{X}(x) \geq u\right\}$, $\operatorname{com} x_{n} \downarrow F_{X}^{q i}(u)$. Como $F_{X}$ é contínua à direita, temos $F_{X}\left(x_{n}\right) \downarrow F_{X}\left(F_{X}^{q i}(u)\right)$; como $u \leq F_{X}\left(x_{n}\right)$ se conclui que $u \leq F_{X}\left(F_{X}^{q i}(u)\right)$.

$(\Rightarrow): F_{X}^{q i}(u) \leq t \Rightarrow F_{X}\left(F_{X}^{q i}(u)\right) \leq F_{X}(t)$, pois $F_{X}$ é não decrescente. E, como $u \leq F_{X}\left(F_{X}^{q i}(u)\right)$, temos que $u \leq F_{X}(t)$.

$$
(\Leftarrow): u \leq F_{X}(t) \Rightarrow t \in\left\{x \in R: F_{X}(x) \geq u\right\} \Rightarrow t \geq F_{X}^{q i}(u)
$$


De modo complementar, introduzimos a seguinte definição e o resultado correspondente.

3.3.3 Definição Seja $F_{X}: R \rightarrow[0,1]$ a função de distribuição acumulada, da variável aleatória $X$. Definamos $\forall u \in(0,1)$, a aplicação $F_{X}^{q i G}$ (quase-inversa generalizada)

$$
\begin{gathered}
F_{X}^{q i G}:(0,1) \rightarrow R \\
F_{X}^{q i G}(u):=\left\{\begin{array} { l } 
{ \xi _ { i } \quad \text { se } u \in ( F _ { X } ( \xi _ { i } ^ { - } ) , F _ { X } ( \xi _ { i } ^ { + } ) ] } \\
{ \operatorname { i n f } \{ x \in R : F _ { X } ( x ) \geq u \} }
\end{array} \quad \text { se } \left\{\begin{array}{l}
u \in\left(F_{X}\left(\xi_{i}^{+}\right), F_{X}\left(\xi_{i+1}^{-}\right)\right] \\
u>F_{X}\left(\xi_{i}^{+}\right), \forall i \\
u \leq F_{X}\left(\xi_{i}^{-}\right), \forall i
\end{array}\right.\right.
\end{gathered}
$$

$\left\{\xi_{i}\right\}_{i=0}^{\infty}$ pontos de descontinuidade da $F_{X}$.

Notação

$$
\left(F_{X}\left(\xi_{i}^{+}\right), F_{X}\left(\xi_{i+1}^{-}\right)\right]=F_{X}\left(\xi_{i+1}^{-}\right) \text {se } F_{X}\left(\xi_{i}^{+}\right)=F_{X}\left(\xi_{i+1}^{-}\right)
$$

Notemos que a função anterior está bem definida $\forall u \in(0,1)$, pois ela coincide bloco a bloco com a quase-inversa da $F_{X}$ (onde ela poderia ser assim definida, pela continuidade). O conjunto de pontos de descontinuidade da $F_{X},\left\{\xi_{i}\right\}_{i=0}^{\infty}$, no máximo enumerável, determina, na imagem da $F_{X}$, intervalos semi-abertos $\left(F_{X}\left(\xi_{i}^{-}\right), F_{X}\left(\xi_{i}^{+}\right)\right]$. Nesses intervalos podemos definir $F_{X}^{q i G} \operatorname{como} \xi_{i}$, já que o objetivo é reobter o valor, em $R$, que foi enviado pela $F_{X}$ até $F_{X}\left(\xi_{i}\right)$.

Para esta nova definição da quase-inversa generalizada ainda continua valendo 3.3.2. Para isso, só é preciso provar

$$
F_{X}^{q i G}(u) \leq t \Leftrightarrow u \leq F_{X}(t), \quad \forall u \in\left(F_{X}\left(\xi_{i}^{-}\right), F_{X}\left(\xi_{i}^{+}\right)\right] .
$$


Seja $u \in\left(F_{X}\left(\xi_{i}^{-}\right), F_{X}\left(\xi_{i}^{+}\right)\right]$,

$(\Rightarrow): \xi_{i}=F_{X}^{q i G}(u) \leq t \Rightarrow F_{X}\left(\xi_{i}\right) \leq F_{X}(t)$, pois $F_{X}$ é não decrescente; como $F_{X}$ é contínua pela direita $F_{X}\left(\xi_{i}\right)=F_{X}\left(\xi_{i}^{+}\right)$e $u \leq F_{X}\left(\xi_{i}^{+}\right)$, logo $u \leq F_{X}(t)$.

$$
\begin{gathered}
(\Leftarrow): u \leq F_{X}(t) \Rightarrow\left\{\begin{array}{l}
\operatorname{se} F_{X}(t) \in\left(F_{X}\left(\xi_{i}^{-}\right), F_{X}\left(\xi_{i}^{+}\right)\right] \quad \Rightarrow t \geq \xi_{i}=F_{X}^{q i G}(u) \\
\operatorname{se} F_{X}(t) \in\left(F_{X}\left(\xi_{i}^{+}\right), F_{X}\left(\xi_{i+1}^{-}\right)\right] \Rightarrow(*)
\end{array}\right. \\
(*)=\left\{\begin{array}{l}
t \in\left\{x \in R: F_{X}(x) \geq F_{X}(t)\right\} \\
\Rightarrow t \geq F_{X}^{q i G}\left(F_{X}(t)\right)>\xi_{i}\left(=F_{X}^{q i G}(u)\right)
\end{array}\right.
\end{gathered}
$$

Notemos que, se $X$ é uma variável aleatória com f.d.a. $F_{X}$, e portanto admite uma quase-inversa generalizada $F_{X}^{q i G}$, então

$$
P\left(X \in\left(F_{X}^{q i G}\left(F_{X}(x)\right), x\right]\right)=F_{X}(x)-F_{X}\left(F_{X}^{q i G}\left(F_{X}(x)\right)\right)=0, \forall x \in R
$$

\subsubsection{Lema}

Seja $F_{X}: R \rightarrow[0,1]$ função de distribuição acumulada contínua, da variável aleatória $X$. Então, $F_{X}\left(F_{X}^{q i}(u)\right)=u, \forall u \in(0,1)$ (a quase-inversa é uma inversa à direita).

\section{Prova de 3.3.4:}

tomemos $u \in(0,1)$. Seja $A_{=u}:=\left\{x \in R: F_{X}(x)=u\right\}$. Como $\lim _{x \rightarrow+\infty} F_{X}(x)=$ $1, \lim _{x \rightarrow-\infty} F_{X}(x)=0$ e $F_{X}$ é contínua, $A_{=u} \neq \emptyset$. Se $A_{=u}$ possuir apenas um elemento, digamos $x_{0}$, então $F_{X}\left(F_{X}^{q i}(u)\right)=F_{X}\left(x_{0}\right)=u$. Se $A_{=u}$ possuir mais que um elemento, então, sendo $F_{X}$ não decrescente, $A_{=u}$ será da forma $A_{=u}=\left[x_{1}, x_{2}\right]$, para 
$x_{1}, x_{2} \in R, x_{1}<x_{2}$. Em conseqüência, $F_{X}\left(F_{X}^{q i}(u)\right)=F_{X}\left(x_{1}\right)=u$. Em qualquer um dos ciois casos, $F_{X}\left(F_{X}^{q i}(u)\right)=u$.

Note que $F: R \rightarrow[0,1]$ é uma função de distribuição acumulada e $F^{q i G}$, sua inversa generalizada. Então, $F\left(F^{q i G}(y)\right) \geq y, \forall y \in(0,1)$. Na verdade, 3.3.4 é parcialmente válido, a saber, se $y \in(0,1)$ pode ser expressado como $y=F(x)$ para algum $x \in R$, então $F\left(F^{q i G}(y)\right)=y$.

\subsubsection{Lema}

Seja $F_{X}: R \rightarrow[0,1]$ função de distribuição acumulada contínua, da variável aleatória $X$. Então, a variável aleatória $F_{X}(X)$ é uniforme em $(0,1)$.

Prova de 3.3.5:

tomemos $u \in(0,1)$

$$
\begin{aligned}
P\left(F_{X}(X) \leq u\right) & =P\left(F_{X}(X)=u\right)+P\left(F_{X}(X)<u\right) \\
& =P\left(F_{X}(X)<u\right) \text { pois } F_{X} \text { é contínua } \\
& =P\left(X<F_{X}^{q i}(u)\right) \text { por } 3.3 .2 \\
& =F_{X}\left(F_{X}^{q i}(u)\right)=u \text { por } 3.3 .4
\end{aligned}
$$


Levando em conta 3.3.2 antes provado é possível demonstrar que:

(i) Dado $-M<0$; supor que $\nexists M_{0} \in R$ tal que $F_{X}\left(M_{0}\right)=0$; logo, $F_{X}(-M)>0$.

Defina $\varepsilon_{-M}:=F_{X}(-M)>0$. Se $u \leq \varepsilon_{-M} \Rightarrow F_{X}^{q i}(u) \leq-M$, pela aplicação de 3.3.2, i.e., é possível fazer a função $F_{X}^{q i}$ arbitrariamente pequena.

Logo, $\lim _{u \rightarrow 0^{+}} F_{X}^{q i}(u)=-\infty$, pela arbitrariedade de $M$.

Defina,

$$
F_{X}^{q i}(0):=-\infty
$$

(ii) Se existir $M_{0} \in R$ tal que $F_{X}\left(M_{0}\right)=0$. Então, $\forall M \leq M_{0}, F_{X}(M)=0$.

Logo, $\left\{x: F_{X}(x)=0\right\} \neq \emptyset$ e $\inf \left\{x: F_{X}(x) \geq 0\right\}=-\infty$. Mais tarde, podemos estender a definição de $F_{X}^{q i}$, designando

$$
F_{X}^{q i}(0):=-\infty
$$

(iii) Dado $M>0$; suponhamos que $\exists M_{1} \in R$ tal que $F_{X}\left(M_{1}\right)=1$; logo, $F_{X}(M)<1$ ou equivalentemente, $1-F_{X}(M)>0$.

Definamos $\varepsilon_{M}:=1-F_{X}(M)>0$. Se $1-u<\varepsilon_{M} \Rightarrow F_{X}^{q i}(u)>M$, pela aplicação de 3.3 .2 , i.e., é possível fazer a função $F_{X}^{q i}$ arbitrariamente grande.

Logo, $\lim _{u \rightarrow 1^{-}} F_{X}^{q i}(u)=+\infty$, pela arbitrariedade de $M$. 
Definamos

$$
F_{X}^{q i}(1):=+\infty
$$

(iv) Se existir $M_{1} \in R$ tal que $F_{X}\left(M_{1}\right)=1$. Então, $\left\{x: F_{X}(x)=1\right\} \neq \emptyset$, e como $\left\{x: F_{X}(x)=1\right\}$ é limitado inferiormente em $R$, pois $F_{X}$ é contínua. Então, pelo axioma da completitude, existe $m:=\inf \left\{x: F_{X}(x)=1\right\} . \mathrm{E} \forall x, x \geq m, F_{X}(x)=1$, i.e., temos um patamar em $[m,+\infty)$.

Definamos

$$
F_{X}^{q i}(1):=m=\inf \left\{x: F_{X}(x)=1\right\}
$$

\subsubsection{Definição}

$$
\begin{aligned}
& F_{X}^{q i}(0)=-\infty \\
& F_{X}^{q i}(1)=\left\{\begin{array}{cc}
\inf \left\{x: F_{X}(x)=1\right\} & \text { se } \exists M: F_{X}(M)=1 \\
+\infty & \text { caso contrário. }
\end{array}\right.
\end{aligned}
$$

notemos que $F_{X}^{q i}$ pode ser extendida continuamente em 0 . Mas nem sempre é possível sua extensão contínua em 1 .

\subsection{7 lema*}

Seja $F_{X}$, f.d.a. contínua, da variável aleatória $X$. Então, $F_{X}^{q i}$ é contínua à direita, $\forall u \in(0,1)$. 
Prova de 3.3.7:

sejam, $u \in(0,1)$ e $\epsilon>0$, arbitrários. Adotemos $\delta_{u, \epsilon} \leq F_{X}\left(\epsilon+F_{X}^{q i}(u)\right)-u$. Logo, $\forall v \in(0,1)$ tal que $v-u<\delta$, então, $F_{X}^{q i}(v)-F_{X}^{q i}(u) \leq \epsilon$.

Pois, se $v-u<\delta \Rightarrow v \leq F_{X}\left(\epsilon+F_{X}^{q i}(u)\right)$ se, e somente, se 3.3.2)

$$
F_{X}^{q i}(v) \leq \epsilon+F_{X}^{q i}(u)
$$

Logo, pela arbitrariedade de $u$ e $\epsilon$, temos a continuidade pela direita.

Prova de 3.2.3:

\subsubsection{Teorema*}

Dado um vetor aleatório $n$-dimensional $\underline{X}=\left(X_{1}, \cdots, X_{n}\right)$ com f.d.a. $F_{\underline{X}}$ e funções de distribuições marginais contínuas $F_{X_{i}}$, a aplicação

$$
C_{\underline{X}}:[0,1]^{n} \rightarrow[0,1]
$$

definida por

$$
C_{\underline{X}}\left(u_{1}, \cdots, u_{n}\right):=F_{\underline{X}}\left(F_{X_{1}}^{q i}\left(u_{1}\right), \cdots, F_{X_{n}}^{q i}\left(u_{n}\right)\right),\left(u_{1}, \cdots, u_{n}\right) \in I^{n}, I=[0,1]
$$

é f.d.a. de marginais univariadas, uniformes. 
Prova de 3.3.8:

previamente é preciso notar que, como as aplicações $F_{X_{i}}^{q i}$ estão bem definidas, então $F_{\underline{X}}$ pode ser aplicada aos pontos de $R^{n}$, da forma $\left(F_{X_{1}}^{q i}\left(u_{1}\right), \cdots, F_{X_{n}}^{q i}\left(u_{n}\right)\right)$, onde $u_{i} \in I, \quad i=1, \cdots, n$.

$$
\begin{aligned}
C_{\underline{X}}\left(0, u_{2}, \cdots, u_{n}\right) & =F_{\underline{X}}\left(F_{X_{1}}^{q i}(0), F_{X_{2}}^{q i}\left(u_{2}\right), \cdots, F_{X_{n}}^{q i}\left(u_{n}\right)\right) \\
& =F_{\underline{X}}\left(-\infty, F_{X_{2}}^{q i}\left(u_{2}\right), \cdots, F_{X_{n}}^{q i}\left(u_{n}\right)\right) \\
& =0
\end{aligned}
$$

enquanto que,

$$
\begin{aligned}
C_{\underline{X}}(1, \cdots, 1) & =F_{\underline{X}}\left(F_{X_{1}}^{q i}(1), \cdots, F_{X_{n}}^{q i}(1)\right) \\
& =1 .
\end{aligned}
$$

Decorre das definições previas das $F_{X_{i}}^{q i}$ nos pontos 0 e 1 .

Notemos que $F_{\underline{X}}$ é continua pela direita e as $\left\{F_{X_{i}}^{q i}\right\}_{i=1}^{n}$, são todas contínuas pela direita (por 3.3.7). Logo, $C_{\underline{X}}$ é contínua pela direita.

Por outro lado, como as $F_{X_{i}}^{q i}$ são não decrescentes, pois as $F_{X_{i}}$ são não decrescentes, e como $F_{\underline{X}}$ é f.d.a. resulta $C_{\underline{X}}\left(\underline{X} \in\left(a_{1}, b_{1}\right] \times \cdots \times\left(a_{n}, b_{n}\right]\right) \geq 0, a_{i} \leq b_{i} i=$ $1, \cdots, n$.

Para demonstrar que as marginais univariadas de $C_{\underline{X}}$ são uniformes $(0,1)$ precisamos notar que, como as marginais acumuladas univariadas são contínuas, resul$\operatorname{tam} F_{X_{i}}\left(X_{i}\right)$ variáveis aleatórias com distribuição $U(0,1), i=1, \cdots, n$ (vide 3.3.5). 
Nota 1: Como $X_{i}$ tem f.d.a. $F_{X_{i}}$ contínua, então $P\left(X_{i}=u\right)=0, \forall u \in R$.

Nota 2: Como $F_{X_{i}}\left(X_{i}\right)$ é $U(0,1)$, pois $F_{X_{i}}$ é contínua, então $P\left(F_{X_{i}}\left(X_{i}\right)=u\right)=$ $0, \forall u \in(0,1)$.

Sem perda da generalidade, provemos para $n=2$, que $C_{\underline{X}}$ tem distribuições marginais uniformes,

$$
\begin{aligned}
C_{X_{1}, X_{2}}\left(u_{1}, u_{2}\right) & =F_{X_{1}, X_{2}}\left(F_{X_{1}}^{q i}\left(u_{1}\right), F_{X_{2}}^{q i}\left(u_{2}\right)\right) \\
\lim _{u_{2} \rightarrow 1} C_{X_{1}, X_{2}}\left(u_{1}, u_{2}\right) & =\lim _{u_{2} \rightarrow 1} F_{X_{1}, X_{2}}\left(F_{X_{1}}^{q i}\left(u_{1}\right), F_{X_{2}}^{q i}\left(u_{2}\right)\right) \\
& =F_{X_{1}, X_{2}}\left(F_{X_{1}}^{q i}\left(u_{1}\right),+\infty\right) \\
& =P\left(X_{1} \leq F_{X_{1}}^{q i}\left(u_{1}\right)\right) \\
& =u_{1}, \quad \text { por } 3.3 .4
\end{aligned}
$$

A prova do caso $n>2$ é obtida só com aplicações reiteradas da anterior.

Notemos que, na prova precedente, assumimos a possibilidade de extender $F_{X_{i}}^{q i}$ no valor 1 , como sendo $+\infty$. Mas a prova é semelhante, quando não for o caso. 


\subsubsection{Proposição* Identificando o acoplamento associado}

Consideremos a função de distribuição acumulada $C_{\underline{X}}$, definida por

$$
C_{\underline{X}}\left(u_{1}, \cdots, u_{n}\right)=F_{\underline{X}}\left(F_{X_{1}}^{q i}\left(u_{1}\right), \cdots, F_{X_{n}}^{q i}\left(u_{n}\right)\right),\left(u_{1}, \cdots, u_{n}\right) \in I^{n}
$$

onde $F_{\underline{X}}$ é a $f . d . a$. do vetor aletório $\underline{X}=\left(X_{1}, \cdots, X_{n}\right)$ de marginais acumuladas contínuas $\left\{F_{X_{i}}\right\}_{i=1}^{n}$. Então, $C_{\underline{X}}$ é a função de distribuição acumulada conjunta, das variáveis aleatórias $F_{X_{1}}\left(X_{1}\right), \cdots, F_{X_{n}}\left(X_{n}\right)$, i. e.,

$P\left(F_{X_{1}}\left(X_{1}\right) \leq u_{1}, \cdots, F_{X_{n}}\left(X_{n}\right) \leq u_{n}\right)=C_{X_{1}, \cdots, X_{n}}\left(u_{1}, \cdots, u_{n}\right), \quad\left(u_{1}, \cdots, u_{n}\right) \in(0,1)^{n}$

Prova

novamente, resolvamos o caso bivariado, sem perda da generalidade.

$$
\begin{aligned}
P\left(F_{X_{1}}\left(X_{1}\right) \leq u_{1}, F_{X_{2}}\left(X_{2}\right) \leq u_{2}\right) \\
=P\left(F_{X_{1}}\left(X_{1}\right) \leq u_{1}, F_{X_{2}}\left(X_{2}\right)<u_{2}\right)+P\left(F_{X_{1}}\left(X_{1}\right) \leq u_{1}, F_{X_{2}}\left(X_{2}\right)=u_{2}\right) \\
=P\left(F_{X_{1}}\left(X_{1}\right) \leq u_{1}, F_{X_{2}}\left(X_{2}\right)<u_{2}\right), \text { pela Nota } 2, i=2 \\
=P\left(F_{X_{1}}\left(X_{1}\right) \leq u_{1}, X_{2}<F_{X_{2}}^{q i}\left(u_{2}\right)\right), \text { desde } 3.3 .2 \text { sobre } X_{2} \\
=P\left(F_{X_{1}}\left(X_{1}\right) \leq u_{1}, X_{2} \leq F_{X_{2}}^{q i}\left(u_{2}\right)\right), \text { pela Nota } 1, i=2 \\
=P\left(X_{1} \leq F_{X_{1}}^{q i}\left(u_{1}\right), X_{2} \leq F_{X_{2}}^{q i}\left(u_{2}\right)\right), 3.3 .2 \text { e Nota } 1,2, i=1 \\
=F_{X_{1}, X_{2}}\left(F_{X_{1}}^{q i}\left(u_{1}\right), F_{X_{2}}^{q i}\left(u_{2}\right)\right) \\
=C_{X_{1}, X_{2}}\left(u_{1}, u_{2}\right) .
\end{aligned}
$$




\subsubsection{Teorema*}

Seja $F_{\underline{X}}$ a função de distribuição acumulada do vetor aleatório $n$-dimensional $\underline{X}=\left(X_{1}, \cdots, X_{n}\right)$, e $\left\{F_{X_{i}}\right\}_{i=1}^{n}$ a sequência de distribuições acumuladas marginais, contínuas. Então, a função de distribuição acumulada, de marginais uniformes, $C_{\underline{X}}: I^{n} \rightarrow I, I=[0,1]$, definida por

$$
C_{\underline{X}}\left(u_{1}, \cdots, u_{n}\right)=F_{\underline{X}}\left(F_{X_{1}}^{q i}\left(u_{1}\right), \cdots, F_{X_{n}}^{q i}\left(u_{n}\right)\right),\left(u_{1}, \cdots, u_{n}\right) \in I^{n}
$$

é o único acoplamento associado a $F_{\underline{X}}$, onde cada $F_{X_{i}}^{q i}$ foi definida anteriormante.

Prova de 3.3.10:

como já verificamos anteriormente, $C_{\underline{X}}$ constitui uma f.d.a. de marginais uniformes $(0,1)$. Precisamos demonstrar que $C_{\underline{X}}$ é um acoplamento associado. Provaremos isto para $n=2$, sem perda da generalidade.

Nota 3: Se $X$ é uma variável aleatória com f.d.a. contínua $F_{X}$, e portanto admite uma quase-inversa $F_{X}^{q i}$, verifica-se

$$
\begin{aligned}
P\left(X \in\left(F_{X}^{q i}\left(F_{X}(x)\right), x\right]\right) & =F_{X}(x)-F_{X}\left(F_{X}^{q i}\left(F_{X}(x)\right)\right) \\
& =0, \forall x \in R
\end{aligned}
$$

é direta conseqüência de 3.3.4. Percebamos que $F^{q i}(F(x)) \leq x, \forall x \in R$.

Consideremos $x_{1}$ e $x_{2}$ valores na imagem de $X_{1}$ e $X_{2}$ respectivamente. 


$$
\begin{aligned}
C_{X_{1}, X_{2}}\left(F_{X_{1}}\left(x_{1}\right), F_{X_{2}}\left(x_{2}\right)\right) \\
=F_{X_{1}, X_{2}}\left(F_{X_{1}}^{q i}\left(F_{X_{1}}\left(x_{1}\right)\right), F_{X_{2}}^{q i}\left(F_{X_{2}}\left(x_{2}\right)\right)\right) \text {, por definição da } C_{X_{1}, X_{2}} \\
=P\left(X_{1} \leq t, X_{2} \leq F_{X_{2}}^{q i}\left(F_{X_{2}}\left(x_{2}\right)\right)\right), t=F_{X_{1}}^{q i}\left(F_{X_{1}}\left(x_{1}\right)\right) \\
=P\left(X_{1} \leq t, X_{2} \leq x_{2}\right)-P\left(X_{1} \leq t, X_{2} \in\left(F_{X_{2}}^{q i}\left(F_{X_{2}}\left(x_{2}\right)\right), x_{2}\right]\right) \\
=P\left(X_{1} \leq t, X_{2} \leq x_{2}\right), \text { pela Nota } 3 \text { aplicada a } X_{2} \\
=P\left(X_{1} \leq F_{X_{1}}^{q i}\left(F_{X_{1}}\left(x_{1}\right)\right), X_{2} \leq x_{2}\right) \\
=P\left(X_{1} \leq x_{1}, X_{2} \leq x_{2}\right)-P\left(X_{1} \in\left(F_{X_{1}}^{q i}\left(F_{X_{1}}\left(x_{1}\right)\right), x_{1}\right], X_{2} \leq x_{2}\right) \\
=P\left(X_{1} \leq x_{1}, X_{2} \leq x_{2}\right), \text { pela Nota } 3 \text { aplicada a } X_{1} \\
=F_{X_{1}, X_{2}}\left(x_{1}, x_{2}\right) .
\end{aligned}
$$

A seguir, demonstraremos a unicidade do acoplamento. Sejam $C_{\underline{X}}$ e $C_{\underline{X}}^{*}$ dois acoplamentos associados a $F_{\underline{X}}$ e consideremos $\left(u_{1}, \cdots, u_{n}\right) \in(0,1)^{n}$.

Notemos que, como cada uma das $F_{X_{i}} i=1, \cdots, n$ é contínua, para todo $u_{i} \in$ $(0,1)$ existe algum $x_{i} \in R$ tal que $u_{i}=F_{X_{i}}\left(x_{i}\right), i=1, \cdots, n$.

$$
\begin{aligned}
C_{\underline{X}}\left(u_{1}, \cdots, u_{n}\right) & =C_{\underline{X}}\left(F_{X_{1}}\left(x_{1}\right), \cdots, F_{X_{n}}\left(x_{n}\right)\right) \text {, pois } F_{X_{i}} \text { são contínuas } \\
& =F_{\underline{X}}\left(x_{1}, \cdots, x_{n}\right), \text { pois } C_{\underline{X}} \text { é um acoplamento associado a } F_{\underline{X}} \\
& =C_{\underline{X}}^{*}\left(F_{X_{1}}\left(x_{1}\right), \cdots, F_{X_{n}}\left(x_{n}\right)\right) \text {, pois } C_{\underline{X}}^{*} \text { é um acop. associado } \\
& =C_{\underline{X}}^{*}\left(u_{1}, \cdots, u_{n}\right), \text { pois } u_{i}=F_{X_{i}}\left(x_{i}\right) .
\end{aligned}
$$


3.3.11 Existência do acoplamento associado, caso puramente discreto. [Joe (1997)]

A seguir, apresentamos uma adaptação da prova presente em [Joe (1997)].

Consideremos as variáveis aleatórias discretas $X_{1}, \cdots, X_{n}$ com valores em $D_{1}, \cdots$, $D_{n}$ respectivamente, onde cada $D_{j}$ é uma seqüência consecutiva de inteiros (por simplicidade). Denotemos por $i_{j}$ os elementos no conjunto $D_{j}$. Sejam $F_{j}\left(i_{j}\right)$ e $f_{j}\left(i_{j}\right)$ a $f . d . a$. e f.d.p. univariadas, respectivamente, para a j-ésima variável. Sejam $P\left(i_{1}, \cdots, i_{n}\right)$ e $p\left(i_{1}, \cdots, i_{n}\right)$ a $f . d . a$. e f.d.p. $n$-variadas, respectivamente. $C_{\underline{X}}$ associado com $P$ precisa satisfazer

$$
P\left(i_{1}, \cdots, i_{n}\right)=C_{\underline{X}}\left(F_{1}\left(i_{1}\right), \cdots, F_{n}\left(i_{n}\right)\right), \quad \forall\left(i_{1}, \cdots, i_{n}\right) \in D_{1} \times \cdots \times D_{n} .
$$

Definimos então, $C_{\underline{X}}$ uniforme no retângulo $\otimes_{1 \leq j \leq n}\left[F_{j}\left(i_{j}-1\right), F_{j}\left(i_{j}\right)\right]$ isto é,

$$
c_{\underline{X}}\left(u_{1}, \cdots, u_{n}\right):= \begin{cases}\frac{p\left(i_{1}, \cdots, i_{n}\right)}{\prod_{j=1}^{n} f_{j}\left(i_{j}\right)} & \text { se, }\left(u_{1}, \cdots, u_{n}\right) \in \otimes_{1 \leq j \leq n}\left[F_{j}\left(i_{j}-1\right), F_{j}\left(i_{j}\right)\right], \\ & \text { para algum }\left(i_{1}, \cdots, i_{n}\right) \in D_{1} \times \cdots \times D_{n} \\ 0 & \text { caso contrário }\end{cases}
$$

é a f.d.p. da distribuição acumulada $C_{\underline{X}}$.

A seguir, verificamos que $C_{\underline{X}}$ é um acoplamento associado com $P$.

Prova de 3.3.11:

provemos para $n=2$, sem perda da generalidade,

$$
P\left(i_{1}, i_{2}\right)=\sum_{s_{1} \in T_{i_{1}}} \sum_{s_{2} \in T_{i_{2}}} p\left(s_{1}, s_{2}\right), \quad T_{i_{k}}:=D_{k} \cap\left(-\infty, i_{k}\right], k=1,2
$$


106

Por outro lado, definamos

$$
\begin{aligned}
& M\left(u_{k}\right):=\left\{t_{k}: t_{k} \in\left[F_{k}\left(i_{k}-1\right), F_{k}\left(i_{k}\right)\right], \forall i_{k} \in D_{k}, t_{k} \leq u_{k}\right\}, \forall u_{k} \in[0,1] \\
& C_{\underline{X}}\left(u_{1}, u_{2}\right)=\sum_{t_{1} \in M\left(u_{1}\right)} \sum_{t_{2} \in M\left(u_{2}\right)} \frac{p\left(i_{1}, i_{2}\right)}{f_{1}\left(i_{1}\right) f_{2}\left(i_{2}\right)} \\
& C_{\underline{X}}\left(F_{1}\left(i_{1}\right), F_{2}\left(i_{2}\right)\right)=\sum_{t_{1} \in M\left(F_{1}\left(i_{1}\right)\right)} \sum_{t_{2} \in M\left(F_{2}\left(i_{2}\right)\right)} \frac{p\left(s_{1}, s_{2}\right)}{f_{1}\left(s_{1}\right) f_{2}\left(s_{2}\right)} \\
&=\sum_{s_{1} \in T_{i_{1}}} f_{1}\left(s_{1}\right) \sum_{s_{2} \in T_{i_{2}}} f_{2}\left(s_{2}\right) \frac{p\left(s_{1}, s_{2}\right)}{f_{1}\left(s_{1}\right) f_{2}\left(s_{2}\right)} \\
&=P\left(i_{1}, i_{2}\right) .
\end{aligned}
$$

Notemos que, considerando a função de distribuição acumulada marginal, a partir do acoplamento, para a primeira variável, ela corresponde a uma $U(0,1)$, pois,

$$
\sum_{i_{2}} \cdots \sum_{i_{n}} \frac{p\left(i_{1}, \cdots, i_{n}\right)}{\prod_{j=1}^{n} f_{j}\left(i_{j}\right)} f_{2}\left(i_{2}\right) \cdots f_{n}\left(i_{n}\right)=\left[f_{1}\left(i_{1}\right)\right]^{-1} \sum_{i_{2}} \cdots \sum_{i_{n}} p\left(i_{1}, \cdots, i_{n}\right)=1 .
$$

Logo, generalizando, as marginais do acoplamento associado correspondem a uma $U(0,1)$.

Os pontos 3.3.8, 3.3.10, 3.3.11 provaram 3.2.3. 
A seguir, apresentamos exemplos de acoplamentos associados a uma certa função de distribuição acumulada.

\subsubsection{Exemplo}

$$
\begin{aligned}
F_{X, Y}(x, y)=\exp \left\{-\left[e^{-x}+e^{-y}-\left(e^{\delta x}+e^{\delta y}\right)^{-\frac{1}{\delta}}\right]\right\},-\infty<x, y<+\infty, \delta>0 \\
F_{X}(x)=\exp \left\{-e^{-x}\right\} \\
F_{Y}(y)=\exp \left\{-e^{-y}\right\} \\
F_{X}^{-1}(u)=-\ln (-\ln (u)), 0<u<1 \\
F_{Y}^{-1}(v)=-\ln (-\ln (v)), 0<v<1 \\
C_{X, Y}(u, v)=\operatorname{logo} \\
C_{X, Y}(u, v)=\operatorname{uvexp}\left\{\left[(-\ln (u))^{-\delta}+(-\ln (v))^{-\delta}\right]^{-\frac{1}{\delta}}\right\}
\end{aligned}
$$

que verifica:

$$
F_{X, Y}(x, y)=C_{X, Y}\left(F_{X}(x), F_{Y}(y)\right)
$$

Generalizando:

$$
\begin{gathered}
F_{\underline{X}}(\underline{x})=\exp \left\{-\left[\sum_{i=1}^{n} e^{-x_{i}}-\left(\sum_{i=1}^{n} e^{\delta x_{i}}\right)^{-\frac{1}{\delta}}\right]\right\},-\infty<x_{i}<+\infty, \delta>0 \\
F_{X_{i}}\left(x_{i}\right)=\exp \left\{-e^{-x_{i}}\right\} \\
F_{X_{i}}^{-1}\left(u_{i}\right)=-\ln \left(-\ln \left(u_{i}\right)\right), 0<u_{i}<1 \\
C_{\underline{X}}(\underline{u})=\prod_{i=1}^{n} u_{i} \exp \left\{\left[\sum_{i=1}^{n}\left(-\ln \left(u_{i}\right)\right)^{-\delta}\right]^{-\frac{1}{\delta}}\right\}
\end{gathered}
$$


que verifica:

$$
F_{\underline{X}}(\underline{x})=C_{\underline{X}}\left(F_{X_{1}}\left(x_{1}\right), \cdots, F_{X_{n}}\left(x_{n}\right)\right)
$$

\subsubsection{Exemplo}

$$
\begin{aligned}
F_{X, Y}(x, y)=1-\left[e^{-\delta x}+e^{-2 \delta y}-e^{-\delta(x+2 y)}\right]^{\frac{1}{\delta}}, 0 \leq x, y, 1 \leq \delta \\
F_{X}(x)=1-e^{-x} \\
F_{Y}(y)=1-e^{-2 y} \\
F_{X}^{-1}(u)=-\ln (1-u), 0<u<1 \\
F_{Y}^{-1}(v)=-\frac{1}{2} \ln (1-v), 0<v<1 \\
\quad \operatorname{logo} \\
C_{X, Y}(u, v)=F_{X, Y}\left(F_{X}^{-1}(u), F_{Y}^{-1}(v)\right), 0<u, v<1 \\
C_{X, Y}(u, v)=1-\left[(1-u)^{\delta}+(1-v)^{\delta}-(1-u)^{\delta}(1-v)^{\delta}\right]^{\frac{1}{\delta}}
\end{aligned}
$$

que verifica:

$$
F_{X, Y}(x, y)=C_{X, Y}\left(F_{X}(x), F_{Y}(y)\right)
$$

\subsubsection{Exemplo}

$$
\begin{aligned}
F_{X, Y}(x, y)=\exp \left\{-\left(e^{-\delta x}+e^{-\delta y}\right)^{\frac{1}{\delta}}\right\},-\infty<x, y<+\infty, 1 \leq \delta \\
F_{X}(x)=\exp \left\{-e^{-x}\right\} \\
F_{Y}(y)=\exp \left\{-e^{-y}\right\} \\
F_{X}^{-1}(u)=-\ln (-\ln (u)), 0<u<1 \\
F_{Y}^{-1}(v)=-\ln (-\ln (v)), 0<v<1 \\
C_{X, Y}(u, v)=\log 0 \\
F_{X, Y}\left(F_{X}^{-1}(u), F_{Y}^{-1}(v)\right), 0<u, v<1
\end{aligned}
$$




$$
C_{X, Y}(u, v)=\exp \left\{-\left(\ln ^{\delta}\left(\frac{1}{u}\right)+\ln ^{\delta}\left(\frac{1}{v}\right)\right)^{\frac{1}{\delta}}\right\}
$$

que verifica:

$$
F_{X, Y}(x, y)=C_{X, Y}\left(F_{X}(x), F_{Y}(y)\right)
$$

Generalizando:

$$
\begin{gathered}
F_{\underline{X}}(\underline{x})=\exp \left\{-\left(\sum_{i=1}^{n} e^{-\delta x_{i}}\right)^{\frac{1}{\delta}}\right\},-\infty<x_{i}<+\infty, 1 \leq \delta \\
F_{X_{i}}\left(x_{i}\right)=\exp \left\{-e^{-x_{i}}\right\} \\
F_{X_{i}}^{-1}\left(u_{i}\right)=-\ln \left(-\ln \left(u_{i}\right)\right), 0<u_{i}<1 \\
C_{\underline{X}}(\underline{u})=\exp \left\{-\left(\sum_{i=1}^{n} \ln ^{\delta}\left(\frac{1}{u_{i}}\right)\right)^{\frac{1}{\delta}}\right\}
\end{gathered}
$$

que verifica:

$$
F_{\underline{X}}(\underline{x})=C_{\underline{X}}\left(F_{X_{1}}\left(x_{1}\right), \cdots, F_{X_{n}}\left(x_{n}\right)\right)
$$

\subsubsection{Exemplo}

$$
\begin{aligned}
F_{X, Y}(x, y)= & \left(1+e^{-x}+e^{-y}\right)^{-1},-\infty<x, y<+\infty \\
F_{X}(x)= & \left(1+e^{-x}\right)^{-1} \\
F_{Y}(y)= & \left(1+e^{-y}\right)^{-1} \\
F_{X}^{-1}(u)= & -\ln \left(\frac{1}{u}-1\right), 0<u<1 \\
F_{Y}^{-1}(v)= & -\ln \left(\frac{1}{v}-1\right), 0<v<1 \\
& \operatorname{logo} \\
C_{X, Y}(u, v)= & F_{X, Y}\left(F_{X}^{-1}(u), F_{Y}^{-1}(v)\right), 0<u, v<1
\end{aligned}
$$




$$
C_{X, Y}(u, v)=\left(\frac{1}{u}+\frac{1}{v}-1\right)^{-1}
$$

que verifica:

$$
F_{X, Y}(x, y)=C_{X, Y}\left(F_{X}(x), F_{Y}(y)\right)
$$

Generalizando:

$$
\begin{gathered}
F_{\underline{X}}(\underline{x})=\left(1+\sum_{i=1}^{n} e^{-x_{i}}\right)^{-1},-\infty<x_{i}<+\infty \\
F_{X_{i}}\left(x_{i}\right)=\left(1+e^{-x_{i}}\right)^{-1} \\
F_{X_{i}}^{-1}\left(u_{i}\right)=-\ln \left(\frac{1}{u_{i}}-1\right), 0<u_{i}<1 \\
C_{\underline{X}}(\underline{u})=\left(\left(\sum_{i=1}^{n} \frac{1}{u_{i}}\right)-(n-1)\right)^{-1}
\end{gathered}
$$

que verifica:

$$
F_{\underline{X}}(\underline{x})=C_{\underline{X}}\left(F_{X_{1}}\left(x_{1}\right), \cdots, F_{X_{n}}\left(x_{n}\right)\right)
$$

\subsubsection{Exemplo}

$$
\begin{gathered}
F_{X, Y}(x, y)=\left(1+x^{-\eta}+y^{-\eta}\right)^{-\frac{1}{\theta}}, \eta>0, \theta>, o<x, y<+\infty \\
F_{X}(x)=\left(1+x^{-\eta}\right)^{-\frac{1}{\theta}} \\
F_{Y}(y)=\left(1+y^{-\eta}\right)^{-\frac{1}{\theta}} \\
F_{X}^{-1}(u)=\left(u^{-\theta}-1\right)^{-\frac{1}{\eta}}, 0<u<1 \\
F_{Y}^{-1}(v)=\left(v^{-\theta}-1\right)^{-\frac{1}{\eta}}, 0<v<1 \\
C_{X, Y}(u, v)=\left(u^{-\theta}+v^{-\theta}-1\right)^{-\frac{1}{\theta}}
\end{gathered}
$$

que satisfaz

$$
F_{X, Y}(x, y)=C_{X, Y}\left(F_{X}(x), F_{Y}(y)\right)
$$




\subsubsection{Exemplo (discreto)}

Assumamos a notação dos acoplamentos no caso puramente discreto.

Suponhamos que $D_{1}:=\{0,1\}$ e $D_{2}:=\{0,1\}$ são os domínios respectivos das variáveis $X$ e $Y$.

Tabela 1: $p(x, y)$

\begin{tabular}{|c|c|c|c|}
\hline \hline $\mathrm{Y} \backslash \mathrm{X}$ & 0 & 1 & $f_{2}(\cdot)$ \\
\hline 0 & $1 / 4$ & $1 / 8$ & $3 / 8$ \\
\hline 1 & $3 / 8$ & $1 / 4$ & $5 / 8$ \\
\hline$f_{1}(\cdot)$ & $5 / 8$ & $3 / 8$ & 1 \\
\hline \hline
\end{tabular}

As probabilidades pontuais fornecidas pela Tabela 1 correspondem a variáveis aleatórias $X$ e $Y$ não independentes (consideremos $x=0, y=1$ ).

Tabela 2: $P(x, y)$

\begin{tabular}{|c|c|c|c|}
\hline \hline $\mathrm{Y} \backslash \mathrm{X}$ & 0 & 1 & $F_{2}(\cdot)$ \\
\hline 0 & $1 / 4$ & $3 / 8$ & $3 / 8$ \\
\hline 1 & $5 / 8$ & 1 & 1 \\
\hline$F_{1}(\cdot)$ & $5 / 8$ & 1 & 1 \\
\hline \hline
\end{tabular}

Tabela 3: $p(x, y) /\left(f_{1}(x) f_{2}(y)\right)$

\begin{tabular}{|c|c|c|}
\hline \hline $\mathrm{Y} \backslash \mathrm{X}$ & 0 & 1 \\
\hline 0 & $64 / 60$ & $8 / 9$ \\
\hline 1 & $24 / 25$ & $64 / 60$ \\
\hline \hline
\end{tabular}

A seguir, fabricamos a densidade do acoplamento associado, na partição fornecida pela imagem da f.d.a. $F_{1}$, para o eixo $x$; pela imagem da $f . d . a . F_{2}$, para o eixo $y$. A saber, 


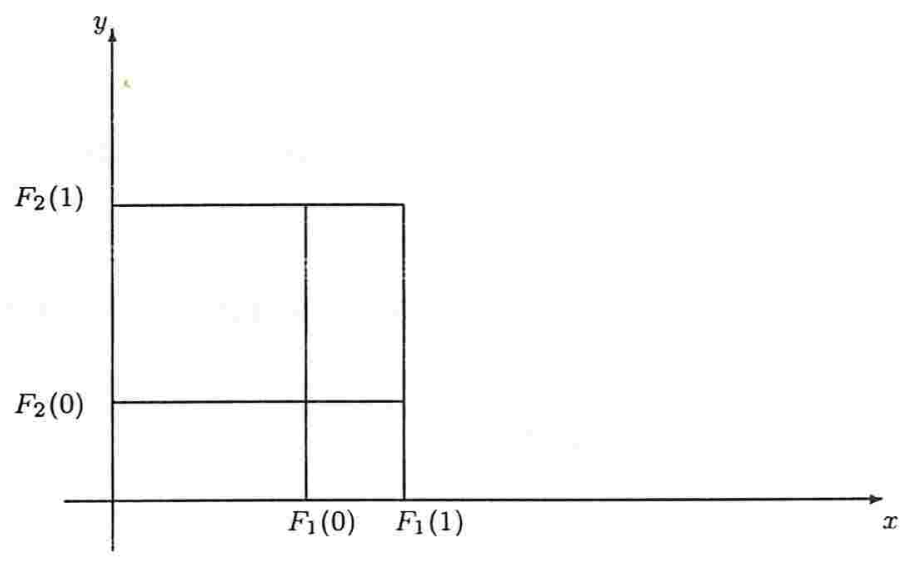

$$
\begin{aligned}
F_{1}(-1)=F_{2}(-1) & =0 \\
F_{1}(0) & =5 / 8 \\
F_{2}(0) & =3 / 8 \\
F_{1}(1)=F_{2}(1) & =1
\end{aligned}
$$

A seguir, apresentamos a densidade $c$ do acoplamento $C$

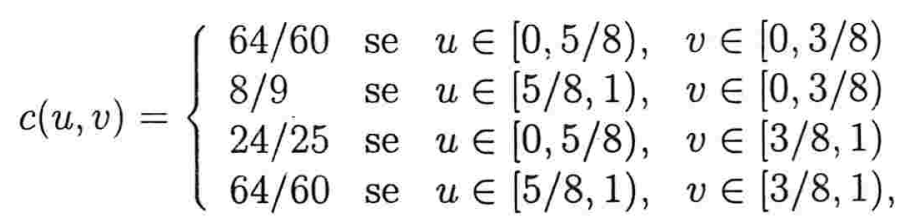

$c$ é definida como 0 no caso contrário. Vemos que a anterior tem marginais uniformes.

Seja $k(x, y)=p(x, y) /\left(f_{1}(x) f_{2}(y)\right)$,

se $u \in\left[F_{1}(-1), F_{1}(0)\right)$,

$$
k(0,0) \frac{3}{8}+k(0,1) \frac{5}{8}=\frac{2}{5}+\frac{3}{5}=1,
$$


onde $\operatorname{Pr}\left(v \in\left[F_{2}(-1), F_{2}(0)\right)\right)=3 / 8 ; \operatorname{Pr}\left(v \in\left[F_{2}(0), F_{2}(1)\right)\right)=5 / 8$.

Se $u \in\left[F_{1}(0), F_{1}(1)\right]$,

$$
k(1,0) \frac{3}{8}+k(1,1) \frac{5}{8}=\frac{1}{3}+\frac{2}{3}=1
$$

Logo, para a primeira variável,

$$
C(u)= \begin{cases}1 & \text { se } u \in[0,1] \\ 0 & c c\end{cases}
$$

O mesmo acontece com a segunda variável.

A partir da densidade prévia, podemos obter a f.d.a. C, acoplamento associado da $P$ original.

$$
C(u, v)=\left\{\begin{array}{lll}
\frac{16}{15} u v & \text { se } u \in[0,5 / 8), & v \in[0,3 / 8) \\
\frac{1}{25} u+\frac{24}{25} u v & \text { se } u \in[5 / 8,1), & v \in[0,3 / 8) \\
\frac{1}{9} v+\frac{8}{9} u v & \text { se } u \in[0,5 / 8), & v \in[3 / 8,1) \\
\frac{1}{15}\{1-u-v\}+\frac{64}{60} u v & \text { se } u \in[5 / 8,1), & v \in[3 / 8,1)
\end{array}\right.
$$

Tabela 4: $C\left(F_{1}(x), F_{2}(y)\right)$

\begin{tabular}{|c|c|c|}
\hline \hline $\mathrm{Y} \backslash \mathrm{X}$ & 0 & 1 \\
\hline 0 & $1 / 4$ & $3 / 8$ \\
\hline 1 & $5 / 8$ & 1 \\
\hline \hline
\end{tabular}

Como as tabelas 2 e 4 são as mesmas, $C$ é acoplạmento associado a $P$. 


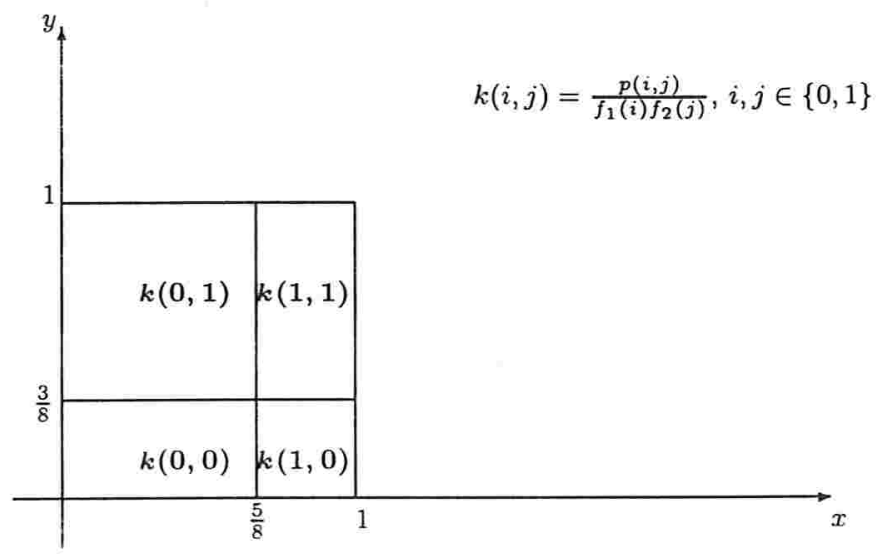

\subsubsection{Exemplo (discreto)}

Suponhamos que $D_{1}=\{0,1,2\}$ e $D_{2}=\{0,1\}$ são os domínios respectivos das variáveis $X$ e $Y$.

Tabela 1: $p(x, y)$

\begin{tabular}{|c|c|c|c|c|}
\hline \hline $\mathrm{Y} \backslash \mathrm{X}$ & 0 & 1 & 2 & $f_{2}(\cdot)$ \\
\hline 0 & .15 & .19 & .15 & .49 \\
\hline 1 & .12 & .21 & .18 & .51 \\
\hline$f_{1}(\cdot)$ & .27 & .40 & .33 & 1 \\
\hline \hline
\end{tabular}

Tabela 2: $P(x, y)$

\begin{tabular}{|c|c|c|c|c|}
\hline \hline $\mathrm{Y} \backslash \mathrm{X}$ & 0 & 1 & 2 & $F_{2}(\cdot)$ \\
\hline 0 & .15 & .34 & .49 & .49 \\
\hline 1 & .27 & .67 & 1 & 1 \\
\hline$F_{1}(\cdot)$ & .27 & .67 & 1 & 1 \\
\hline \hline
\end{tabular}


Tabela 3: $p(x, y) /\left(f_{1}(x) f_{2}(y)\right)$

\begin{tabular}{|c|c|c|c|}
\hline \hline $\mathrm{Y} \backslash \mathrm{X}$ & 0 & 1 & 2 \\
\hline 0 & 1.13 & .97 & .93 \\
\hline 1 & .87 & 1.03 & 1.07 \\
\hline \hline
\end{tabular}

A seguir, fabricamos a densidade do acoplamento associado, na partição fornecida pela imagem da f.d.a. $F_{1}$, para o eixo $x$; pela imagem da f.d.a. $F_{2}$, para o eixo $y$. A saber,

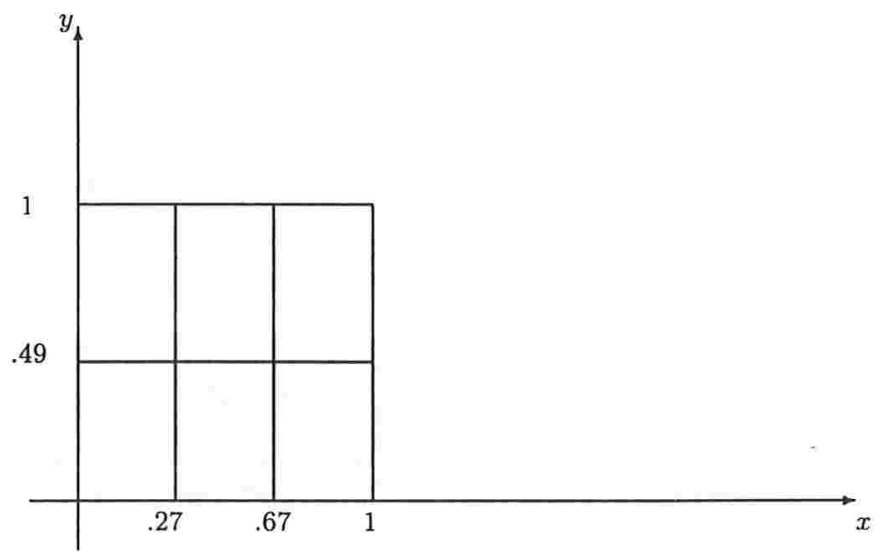

A seguir, apresentamos a densidade $c$ do acoplamento $C$

$$
c(u, v)=\left\{\begin{array}{lll}
1.13 & \text { se } u \in[0, .27), & v \in[0, .49) \\
.87 & \text { se } u \in[0, .27), & v \in[.49,1] \\
.97 & \text { se } u \in[.27, .67), & v \in[0, .49) \\
1.03 & \text { se } u \in[.27, .67), & v \in[.49,1] \\
.93 & \text { se } u \in[.67,1], & v \in[0, .49) \\
1.07 & \text { se } u \in[.67,1], & v \in[.49,1]
\end{array}\right.
$$

$c$ é definida como 0 no caso contrário. 


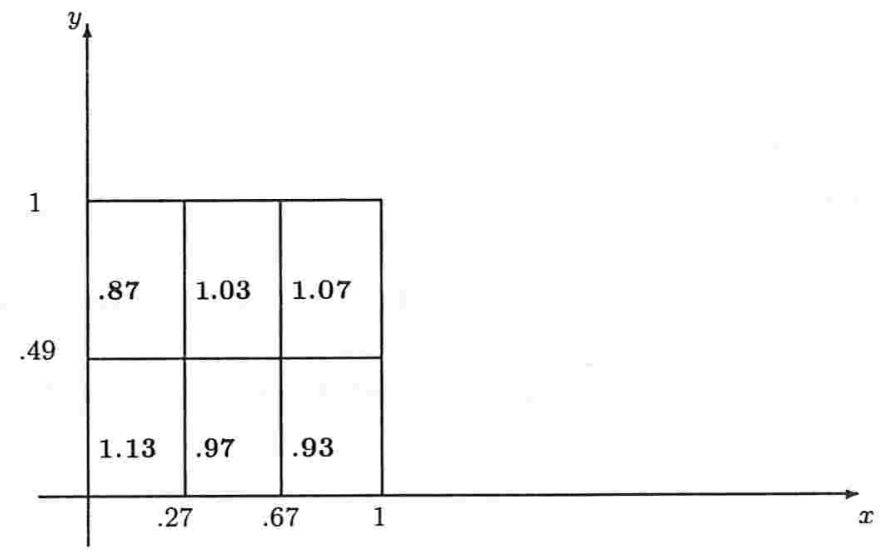

A partir da densidade prévia, podemos obter a f.d.a. C, acoplamento associado da $P$ original.

$$
C(u, v)=\left\{\begin{array}{lll}
1.13 u v & \text { se } u \in[0, .27), & v \in[0, .49) \\
-.13 u+.87 u v & \text { se } u \in[0, .27), & v \in[.49,1] \\
.04 v+.97 u v & \text { se } u \in[.27, .67), & v \in[0, .49) \\
.04-.03 u-.04 v+1.03 u v & \text { se } u \in[.27, .67), & v \in[.49,1] \\
.07 v+.93 u v & \text { se } u \in[.67,1], & v \in[0, .49) \\
.07-.07 u-.07 v+1.07 u v & \text { se } u \in[.67,1], & v \in[.49,1]
\end{array}\right.
$$

Tabela 4: $C\left(F_{1}(x), F_{2}(y)\right)$

\begin{tabular}{|c|c|c|c|}
\hline \hline $\mathrm{Y} \backslash \mathrm{X}$ & 0 & 1 & 2 \\
\hline 0 & .15 & .34 & .49 \\
\hline 1 & .27 & .67 & 1 \\
\hline \hline
\end{tabular}

Como as tabelas 2 e 4 são iguais, $C$ é acoplamento associado a $P$. 
Densidade acumulada, seu acoplamento e a condição $T P_{2}\left(R R_{2}\right)$

É preciso fazer um comentário deste tópico, que consolida a importância do estudo dos Núcleos Positivos, que correspondem à generalização das transformações $T P_{2}$ ou $R R_{2}$ [ Karlin (1968)].

\subsubsection{Definição}

Uma função $K: R^{2} \rightarrow R$ é Sinal-Regular de ordem $r\left(S R_{r}\right)$ se existe uma seqüência de números $\left\{\epsilon_{m}\right\}_{m}$, onde $\epsilon_{m} \in\{-1,+1\}$ tais que,

$$
\epsilon_{m} K\left(\begin{array}{c}
x_{1}, x_{2}, \cdots, x_{m} \\
y_{1}, y_{2}, \cdots, y_{m}
\end{array}\right) \geq 0, \quad 1 \leq m \leq r
$$

dizemos que é Sinal-Regular de Ordem r Estrita $\left(S S R_{r}\right)$ quando a desigualdade anterior é estrita.

Seja $\epsilon_{m}(K):=\epsilon_{m}$, sinal (constante) do determinante da $m$-ésima ordem, na desigualdade anterior.

No caso em que $\epsilon_{m}=(-1)^{m(m-1) / 2}$, dizemos que $K$ é Sinal-Regra Reversa de Ordem $r\left(R R_{r}\right)$. 


\subsubsection{Teorema [Karlin(1968)]}

Sejam $K: R^{2} \rightarrow R$ uma aplicação $S R_{r}$ sobre $R^{2}, \varphi, \psi$ duas aplicações de $R$ em $R$, que preservam o mesmo sinal (constante) em $R$. Então,

(a) $L\left(x_{1}, x_{2}\right):=\varphi\left(x_{1}\right) \psi\left(x_{2}\right) K\left(x_{1}, x_{2}\right)$ é $S R_{r}$.

(b) Se $\varphi^{-1}$ e $\psi^{-1}$ são aplicações estritamente crescentes,

$$
L(u, v):=K[\varphi(u), \psi(v)], \quad u \in R, v \in R,
$$

é uma função $S R_{r}$ e $\epsilon_{m}(K)=\epsilon_{m}(L)$ para $m=1, \cdots, r$.

Se $\varphi$ é estritamente crescente e $\psi$ estritamente decrescente, então

$L(u, v)$ é $S R_{r}$ e $\epsilon_{m}(K)=(-1)^{m(m-1) / 2} \epsilon_{m}(L)$ para $m=1, \cdots, r$.

A seguir, apresentamos uma adaptação da prova de (b), presente em [Karlin(1968)].

$$
\begin{aligned}
& \epsilon_{m} L\left(\begin{array}{c}
u_{1}, u_{2}, \cdots, u_{m} \\
v_{1}, v_{2}, \cdots, v_{m}
\end{array}\right)=\epsilon_{m}\left|\begin{array}{cc}
L\left(u_{1}, v_{1}\right) & L\left(u_{1}, v_{2}\right) \cdots L\left(u_{1}, v_{m}\right) \\
L\left(u_{2}, v_{1}\right) & L\left(u_{2}, v_{2}\right) \cdots L\left(u_{2}, v_{m}\right) \\
\vdots & \\
L\left(u_{m}, v_{1}\right) & L\left(u_{m}, v_{2}\right) \cdots L\left(u_{m}, v_{m}\right)
\end{array}\right| \\
& =\epsilon_{m}\left|\begin{array}{cc}
K\left(\varphi\left(u_{1}\right), \psi\left(v_{1}\right)\right) & K\left(\varphi\left(u_{1}\right), \psi\left(v_{2}\right)\right) \cdots K\left(\varphi\left(u_{1}\right), \psi\left(v_{m}\right)\right) \\
K\left(\varphi\left(u_{2}\right), \psi\left(v_{1}\right)\right) & K\left(\varphi\left(u_{2}\right), \psi\left(v_{2}\right)\right) \cdots K\left(\varphi\left(u_{2}\right), \psi\left(v_{m}\right)\right) \\
\vdots & \vdots \\
K\left(\varphi\left(u_{m}\right), \psi\left(v_{1}\right)\right) & K\left(\varphi\left(u_{m}\right), \psi\left(v_{2}\right)\right) \cdots K\left(\varphi\left(u_{m}\right), \psi\left(v_{m}\right)\right)
\end{array}\right| \\
& =\epsilon_{m} K\left(\begin{array}{c}
\varphi\left(u_{1}\right), \varphi\left(u_{2}\right), \cdots, \varphi\left(u_{m}\right) \\
\psi\left(v_{1}\right), \psi\left(v_{2}\right), \cdots, \psi\left(v_{m}\right)
\end{array}\right)
\end{aligned}
$$


Acoplamentos e Associação

$=\epsilon_{m} K\left(\begin{array}{c}p_{1}, p_{2}, \cdots, p_{m} \\ q_{1}, q_{2}, \cdots, q_{m}\end{array}\right) \geq 0$

sempre que $p_{i}:=\varphi\left(u_{i}\right)$ e $q_{i}:=\psi\left(v_{i}\right), \quad i=1, \cdots, m$,

pois $u_{1}<u_{2}<\cdots<u_{m}, v_{1}<v_{2}<\cdots<v_{m}$ e $\varphi^{-1}, \psi^{-1}$ são aplicações estritamente crescentes. Como $K$ é $S R_{r}$ conclui-se a última desigualdade.

\subsubsection{Aplicação da existência do acoplamento}

Seja $F_{\underline{X}} f . d . a$. do vetor aleatório $\underline{X}=\left(X_{1}, X_{2}\right)$ com marginais acumuladas $F_{X_{1}}$ e $F_{X_{2}}$, e suponhamos que existem $F_{X_{1}}^{-1}$ e $F_{X_{2}}^{-1}$, respectivamente. Então, existe um único acoplamento associado $C_{\underline{X}}$ tal que,

$$
F_{\underline{X}}\left(x_{1}, x_{2}\right)=C_{\underline{X}}\left(F_{X_{1}}\left(x_{1}\right), F_{X_{2}}\left(x_{2}\right)\right) .
$$

Logo, se $C_{\underline{X}}$ é $S R_{r}$ então, $F_{\underline{X}}$ é $S R_{r}$.

Comentário: Se consideramos como mais simples a análise feita sobre distribuições em $R^{2}$ de marginais uniformes em $(0,1)$, então, pela aplicação anterior resultará mais simples identificar a presência de propriedades vinculadas com $T P_{2}$ e $R R_{2}$, desde os acoplamentos associados que a partir da própria distribuição considerada. 


\subsection{Comportamento da medida $\tau$-Kendall}

Nesta Seção vamos apresentar as provas de 3.2.6 e 3.2.8 [Nelsen (1992)].

Lembremos a seguir os resultados obtidos.

Sejam $\left(X_{1}^{\prime}, X_{2}^{\prime}\right)$ e $\left(X_{1}^{\prime \prime}, X_{2}^{\prime \prime}\right)$ dois pares de vetores aleatórios independentes, ambos com f.d.a. $F_{X_{1}, X_{2}}$.

Se as marginais acumuladas $F_{X_{1}}$ e $F_{X_{2}}$ são contínuas, então

$$
\tau_{X_{1}, X_{2}}=2 P\left\{\left(X_{1}^{\prime}-X_{1}^{\prime \prime}\right)\left(X_{2}^{\prime}-X_{2}^{\prime \prime}\right) \geq 0\right\}-1
$$

Em geral, ante a ausência da hipótese das marginais contínuas, a medida $\tau$ terá a seguinte expressão,

$$
\begin{aligned}
\tau_{X_{1}, X_{2}}= & 2 P\left(X_{1}^{\prime} \leq X_{1}^{\prime \prime}, X_{2}^{\prime} \leq X_{2}^{\prime \prime}\right)-P\left(X_{1}^{\prime} \leq X_{1}^{\prime \prime}\right) \\
& -P\left(X_{2}^{\prime} \leq X_{2}^{\prime \prime}\right)+1+P\left(X_{1}^{\prime}=X_{1}^{\prime \prime}, X_{2}^{\prime}>X_{2}^{\prime \prime}\right) \\
& +P\left(X_{1}^{\prime}>X_{1}^{\prime \prime}, X_{2}^{\prime}=X_{2}^{\prime \prime}\right) \\
& -P\left(X_{1}^{\prime}>X_{1}^{\prime \prime}, X_{2}^{\prime}<X_{2}^{\prime \prime}\right)-P\left(X_{1}^{\prime}<X_{1}^{\prime \prime}, X_{2}^{\prime}>X_{2}^{\prime \prime}\right),
\end{aligned}
$$

esta última expressão facilita o cálculo computacional da medida, no caso discreto. 
3.4.1 Prova de 3.2.6:

(i) :

$$
\begin{aligned}
P\left(X_{1}^{\prime}>X_{1}^{\prime \prime}, X_{2}^{\prime}>X_{2}^{\prime \prime}\right)= & P\left(X_{1}^{\prime} \leq X_{1}^{\prime \prime}, X_{2}^{\prime} \leq X_{2}^{\prime \prime}\right) \\
& -P\left(X_{1}^{\prime} \leq X_{1}^{\prime \prime}\right)-P\left(X_{2}^{\prime} \leq X_{2}^{\prime \prime}\right)+1
\end{aligned}
$$

e como as $F_{X_{i}}$ são contínuas

$P\left\{\left(X_{1}^{\prime}-X_{1}^{\prime \prime}\right)\left(X_{2}^{\prime}-X_{2}^{\prime \prime}\right) \geq 0\right\}=P\left(X_{1}^{\prime} \leq X_{1}^{\prime \prime}, X_{2}^{\prime} \leq X_{2}^{\prime \prime}\right)+P\left(X_{1}^{\prime}>X_{1}^{\prime \prime}, X_{2}^{\prime}>X_{2}^{\prime \prime}\right)$

$\operatorname{logo}$

$$
\begin{aligned}
P\left\{\left(X_{1}^{\prime}-X_{1}^{\prime \prime}\right)\left(X_{2}^{\prime}-X_{2}^{\prime \prime}\right) \geq 0\right\}= & 2 P\left(X_{1}^{\prime} \leq X_{1}^{\prime \prime}, X_{2}^{\prime} \leq X_{2}^{\prime \prime}\right) \\
& -P\left(X_{1}^{\prime} \leq X_{1}^{\prime \prime}\right)-P\left(X_{2}^{\prime} \leq X_{2}^{\prime \prime}\right)+1
\end{aligned}
$$

$$
\begin{aligned}
P\{ & \left.\left(X_{1}^{\prime}-X_{1}^{\prime \prime}\right)\left(X_{2}^{\prime}-X_{2}^{\prime \prime}\right) \geq 0\right\} \\
= & \int_{-\infty}^{+\infty} \int_{-\infty}^{+\infty} P\left\{\left(X_{1}^{\prime}-X_{1}^{\prime \prime}\right)\left(X_{2}^{\prime}-X_{2}^{\prime \prime}\right) \geq 0 \mid X_{1}^{\prime \prime}=x_{1}^{\prime \prime}, X_{2}^{\prime \prime}=x_{2}^{\prime \prime}\right\} d F_{X_{1}, X_{2}}\left(x_{1}^{\prime \prime}, x_{2}^{\prime \prime}\right) \\
= & \int_{-\infty}^{+\infty} \int_{-\infty}^{+\infty}\left\{2 P\left(X_{1}^{\prime} \leq x_{1}^{\prime \prime}, X_{2}^{\prime} \leq x_{2}^{\prime \prime}\right)\right. \\
& \left.-P\left(X_{1}^{\prime} \leq x_{1}^{\prime \prime}\right)-P\left(X_{2}^{\prime} \leq x_{2}^{\prime \prime}\right)+1\right\} d F_{X_{1}, X_{2}}\left(x_{1}^{\prime \prime}, x_{2}^{\prime \prime}\right) \\
= & 2 \int_{-\infty}^{+\infty} \int_{-\infty}^{+\infty} F_{X_{1}, X_{2}}\left(x_{1}, x_{2}\right) d F_{X_{1}, X_{2}}\left(x_{1}, x_{2}\right)+1 \\
& -\int_{-\infty}^{+\infty} F_{X_{1}}\left(x_{1}\right) d F_{X_{1}}\left(x_{1}\right)-\int_{-\infty}^{+\infty} F_{X_{2}}\left(x_{2}\right) d F_{X_{2}}\left(x_{2}\right)
\end{aligned}
$$

notemos que é possível substituir a integral pela somatória, se for pertinente. Agora, como $F_{X_{1}, X_{2}}$ é contínua, as respectivas marginais $F_{X_{1}}$ e $F_{X_{2}}$ são contínuas, portanto 
vale 3.3.5, para cada uma delas. Assim, como a variável aleatória $F_{X_{i}}\left(X_{i}\right)$ é $U(0,1)$, para $i=1,2$; resulta,

$$
\int_{-\infty}^{+\infty} F_{X_{i}}(x) d F_{X_{i}}(x)=\int_{0}^{1} u d u=1 / 2, \quad i=1,2 .
$$

Então

$$
P\left\{\left(X_{1}^{\prime}-X_{1}^{\prime \prime}\right)\left(X_{2}^{\prime}-X_{2}^{\prime \prime}\right) \geq 0\right\}=2 \int_{-\infty}^{+\infty} \int_{-\infty}^{+\infty} F_{X_{1}, X_{2}}\left(x_{1}, x_{2}\right) d F_{X_{1}, X_{2}}\left(x_{1}, x_{2}\right),
$$

e, conseqüentemente

$$
\tau_{X_{1}, X_{2}}=4 \int_{-\infty}^{+\infty} \int_{-\infty}^{+\infty} F_{X_{1}, X_{2}}\left(x_{1}, x_{2}\right) d F_{X_{1}, X_{2}}\left(x_{1}, x_{2}\right)-1
$$

(ii) : Considerando o acoplamento associado à f.d.a., temos

$$
F_{X_{1}, X_{2}}\left(x_{1}, x_{2}\right)=C_{X_{1}, X_{2}}\left(F_{X_{1}}\left(x_{1}\right), F_{X_{2}}\left(x_{2}\right)\right), \forall x_{1}, x_{2}
$$

Como $F_{X_{1}, X_{2}}$ é absolutamente contínua, então $c_{X_{1}, X_{2}}$, verifica,

$$
c_{X_{1}, X_{2}}(u, v):=\frac{\partial^{2} C_{X_{1}, X_{2}}(u, v)}{\partial u \partial v} \forall u, v \in[0,1]
$$

é a densidade do acoplamento associado à $F_{X_{1}, X_{2}}$ e

$$
f_{X_{1}, X_{2}}\left(x_{1}, x_{2}\right)=c\left(F_{X_{1}}\left(x_{1}\right), F_{X_{2}}\left(x_{2}\right)\right) f_{X_{1}}\left(x_{1}\right) f_{X_{2}}\left(x_{2}\right), \quad \forall x_{1}, x_{2}
$$

onde $f_{X_{1}}$ e $f_{X_{2}}$ são as funções de distribuição de probabilidade marginais, para $X_{1}$ e $X_{2}$ respectivamente.

A partir da primeira parte da proposição, e fazendo as substituições,

$$
\begin{gathered}
u:=F_{X_{1}}\left(x_{1}\right), v:=F_{X_{2}}\left(x_{2}\right) ; d u=f_{X_{1}}\left(x_{1}\right) d x_{1}, d v=f_{X_{2}}\left(x_{2}\right) d x_{2} \text {, respectivamente, } \\
\int_{R^{2}} F_{X_{1}, X_{2}}\left(x_{1}, x_{2}\right) d F_{X_{1}, X_{2}}\left(x_{1}, x_{2}\right)=\int_{[0,1]^{2}} C_{X_{1}, X_{2}}\left(x_{1}, x_{2}\right) d C_{X_{1}, X_{2}}\left(x_{1}, x_{2}\right) .
\end{gathered}
$$


$\tau_{X_{1}, X_{2}}$ como medida de associação $T P_{2}\left(R R_{2}\right)$

O Teorema de Nelsen do ano de 1992, apresentado no tópico 3.2.8, esclarece a importância da medida $\tau$ de Kendall como uma medida de associação positiva (negativa), resultante da condição $T P_{2}\left(R R_{2}\right)$ sobre a função de densidade de probabilidade conjunta e/ou a propria função acumulada de distribuição conjunta.

O resultado oferece duas versões. A primeira delas exige que a f.d.p. do par de variáveis envolvidas seja $T P_{2}\left(R R_{2}\right)$, enquanto que a segunda impõe condições sobre a f.d.a. do par de variáveis.

\subsubsection{Prova de 3.2.8:}

para a prova de $(i)$ é preciso recorrer à existência do acoplamento $C_{X_{1}, X_{2}}$ e sua densidade $c_{X_{1}, X_{2}}$ associadas a $F_{X_{1}, X_{2}}$, e lembrar que

$$
\begin{aligned}
F_{X_{1}, X_{2}}\left(x_{1}, x_{2}\right) & =C_{X_{1}, X_{2}}\left(F_{X_{1}}\left(x_{1}\right), F_{X_{2}}\left(x_{2}\right)\right) \\
c_{X_{1}, X_{2}}(u, v) & =\frac{\partial^{2}}{\partial u \partial v} C_{X_{1}, X_{2}}(u, v), \text { densidade do acoplamento } \\
f_{X_{1}, X_{2}}\left(x_{1}, x_{2}\right) & =c_{X_{1}, X_{2}}\left(F_{X_{1}}\left(x_{1}\right), F_{X_{2}}\left(x_{2}\right)\right) f_{X_{1}}\left(x_{1}\right) f_{X_{2}}\left(x_{2}\right)
\end{aligned}
$$

onde $f_{X_{i}}$ é a f.d.p. marginal univariada da v.a. $X_{i}, i=1,2$.

Fazendo a troca de variáveis $u=F_{X_{1}}\left(x_{1}\right)$ e $v=F_{X_{2}}\left(x_{2}\right)$, temos

$$
\begin{aligned}
T_{X_{1}, X_{2}}= & \int_{0}^{1} \int_{0}^{1} \int_{0}^{v_{2}} \int_{0}^{u_{2}}\left[c_{X_{1}, X_{2}}\left(u_{2}, v_{2}\right) c_{X_{1}, X_{2}}\left(u_{1}, v_{1}\right)\right. \\
& \left.-c_{X_{1}, X_{2}}\left(u_{1}, v_{2}\right) c_{X_{1}, X_{2}}\left(u_{2}, v_{1}\right)\right] d u_{1} d v_{1} d u_{2} d v_{2} .
\end{aligned}
$$


Seja

$$
\begin{aligned}
T_{X_{1}, X_{2}}\left(u_{2}, v_{2}\right):= & \int_{0}^{v_{2}} \int_{0}^{u_{2}}\left[c_{X_{1}, X_{2}}\left(u_{2}, v_{2}\right) c_{X_{1}, X_{2}}\left(u_{1}, v_{1}\right)\right. \\
& \left.-c_{X_{1}, X_{2}}\left(u_{1}, v_{2}\right) c_{X_{1}, X_{2}}\left(u_{2}, v_{1}\right)\right] d u_{1} d v_{1} .
\end{aligned}
$$

Segue-se que,

$$
\begin{aligned}
& T_{X_{1}, X_{2}}\left(u_{2}, v_{2}\right) \\
& \quad=C_{X_{1}, X_{2}}\left(u_{2}, v_{2}\right) \frac{\partial^{2}}{\partial u \partial v} C_{X_{1}, X_{2}}\left(u_{2}, v_{2}\right)-\frac{\partial}{\partial u} C_{X_{1}, X_{2}}\left(u_{2}, v_{2}\right) \frac{\partial}{\partial v} C_{X_{1}, X_{2}}\left(u_{2}, v_{2}\right) .
\end{aligned}
$$

Logo,

$$
\begin{aligned}
T_{X_{1}, X_{2}}= & \int_{0}^{1} \int_{0}^{1} C_{X_{1}, X_{2}}(u, v) \frac{\partial^{2}}{\partial u \partial v} C_{X_{1}, X_{2}}(u, v) d u d v \\
& -\int_{0}^{1} \int_{0}^{1} \frac{\partial}{\partial u} C_{X_{1}, X_{2}}(u, v) \frac{\partial}{\partial v} C_{X_{1}, X_{2}}(u, v) d u d v
\end{aligned}
$$

A primeira integral dupla é igual a $\frac{1}{4}\left(\tau_{X_{1}, X_{2}}+1\right)$ entanto a segunda é $\frac{1}{4}\left(1-\tau_{X_{1}, X_{2}}\right)$, esta última verifica-se mediante integração por partes.

$$
\begin{aligned}
& \int_{0}^{1} \frac{\partial}{\partial u} C_{X_{1}, X_{2}}(u, v) \frac{\partial}{\partial v} C_{X_{1}, X_{2}}(u, v) d u \\
& =\left.\frac{\partial}{\partial v} C_{X_{1}, X_{2}}(u, v) C_{X_{1}, X_{2}}(u, v)\right|_{u=0}{ }^{1}-\int_{0}^{1} C_{X_{1}, X_{2}}(u, v) \frac{\partial^{2}}{\partial u \partial v} C_{X_{1}, X_{2}}(u, v) d u \\
& =\frac{\partial}{\partial v} C_{X_{2}}(v) C_{X_{2}}(v)-\int_{0}^{1} C_{X_{1}, X_{2}}(u, v) \frac{\partial^{2}}{\partial u \partial v} C_{X_{1}, X_{2}}(u, v) d u
\end{aligned}
$$

Logo,

$$
\begin{aligned}
\int_{0}^{1} \frac{\partial}{\partial v} C_{X_{2}}(v) C_{X_{2}}(v) d v & =\frac{1}{2}, \text { acoplamento tem marginais } U(0,1) \\
\int_{0}^{1} \int_{0}^{1} C_{X_{1}, X_{2}}(u, v) \frac{\partial^{2}}{\partial u \partial v} C_{X_{1}, X_{2}}(u, v) d u d v & =\frac{1}{4}\left\{\tau_{X_{1}, X_{2}}+1\right\}, \text { por 3.2.6. }
\end{aligned}
$$

Finalmente, $T_{X_{1}, X_{2}}=\frac{\tau_{X_{1}, X_{2}}}{2}$. 
Para provar (ii) notemos que se rearranjamos os termos em $T_{X_{1}, X_{2}}$,

$$
\begin{aligned}
T_{X_{1}, X_{2}}= & \lim _{\|P\| \rightarrow 0} \sum_{p=2}^{n} \sum_{q=2}^{m}\left[C_{X_{1}, X_{2}}\left(u_{p}, v_{q}\right) C_{X_{1}, X_{2}}\left(u_{p-1}, v_{q-1}\right)\right. \\
& \left.-C_{X_{1}, X_{2}}\left(u_{p-1}, v_{q}\right) C_{X_{1}, X_{2}}\left(u_{p}, v_{q-1}\right)\right] .
\end{aligned}
$$

Consideremos a identidade $2(a d-b c)=(a+b+c+d)(a-b-c+d)-\left(a^{2}-b^{2}-c^{2}+d^{2}\right)$ e denotemos $C_{p, q}:=C_{X_{1}, X_{2}}\left(u_{p}, v_{q}\right)$,

conclui-se que

$2 T_{X_{1}, X_{2}}=\lim _{\|P\| \rightarrow 0} \sum_{p=1}^{n} \sum_{q=1}^{m}\left[\left(C_{p, q}+C_{p-1, q}+C_{p, q-1}+C_{p-1, q-1}\right)\left(C_{p, q}-C_{p-1, q}-\right.\right.$ $\left.\left.C_{p, q-1}+C_{p-1, q-1}\right)-\left(C_{p, q}^{2}-C_{p-1, q}^{2}-C_{p, q-1}^{2}+C_{p-1, q-1}^{2}\right)\right]$,

mas, $C_{X_{1}, X_{2}}$ é contínua em cada retângulo $J_{p} \times K_{q}$ e pelo teorema do valor intermediário poderemos garantir que para cada $J_{p} \times K_{q}$ existe $\left(u_{p}^{*}, v_{q}^{*}\right) \in J_{p} \times K_{q}$ tal que

$$
4 C_{X_{1}, X_{2}}\left(u_{p}^{*}, v_{q}^{*}\right)=C_{p, q}+C_{p-1, q}+C_{p, q-1}+C_{p-1, q-1}
$$

Se $\Delta C_{p, q}:=C_{p, q}-C_{p-1, q}-C_{p, q-1}+C_{p-1, q-1}$, representa a probabilidade atribuída a $J_{p} \times K_{q}$ pelo acoplamento $C_{X_{1}, X_{2}}$. Logo,

$$
\begin{aligned}
2 T_{X_{1}, X_{2}}= & \lim _{\|P\| \rightarrow 0}\left\{\sum_{p=1}^{n} \sum_{q=1}^{m} 4 C_{X_{1}, X_{2}}\left(u_{p}^{*}, v_{q}^{*}\right) \Delta C_{p, q}\right. \\
& \left.-\sum_{p=1}^{n} \sum_{q=1}^{m}\left[\left(C_{p, q}^{2}-C_{p-1, q}^{2}-C_{p, q-1}^{2}+C_{p-1, q-1}^{2}\right)\right]\right\} .
\end{aligned}
$$

Como $\lim _{\|P\| \rightarrow 0} \sum_{p=1}^{n} \sum_{q=1}^{m}\left[\left(C_{p, q}^{2}-C_{p-1, q}^{2}-C_{p, q-1}^{2}+C_{p-1, q-1}^{2}\right)\right]=1$, temos que $2 T_{X_{1}, X_{2}}$ é o limite de uma soma de Riemann para

$$
\tau_{X_{1}, X_{2}}=4 \int_{I^{2}} C_{X_{1}, X_{2}}(u, v) d C_{X_{1}, X_{2}}(u, v)-1
$$


Assim, provamos que $T_{X_{1}, X_{2}}=\frac{1}{2} \tau_{X_{1}, X_{2}}$; sempre que $\tau_{X_{1}, X_{2}}$ seja dado pela fórmula,

$$
4 \int_{[0,1]^{2}} C_{X_{1}, X_{2}}\left(u_{1}, u_{2}\right) d C_{X_{1}, X_{2}}\left(u_{1}, u_{2}\right)-1
$$

i.e., no caso absolutamente contínuo. 
3.4.3 Observação (i) Nem sempre a medida de concordância $\tau$ representa uma medida de associação $T P_{2}\left(R R_{2}\right)$.

Medida $\tau$ para variáveis aleatórias discretas,

$$
\begin{aligned}
\tau_{X_{1}, X_{2}}= & 2 P\left(X_{1}^{\prime} \leq X_{1}^{\prime \prime}, X_{2}^{\prime} \leq X_{2}^{\prime \prime}\right)-P\left(X_{1}^{\prime} \leq X_{1}^{\prime \prime}\right) \\
& -P\left(X_{2}^{\prime} \leq X_{2}^{\prime \prime}\right)+1+P\left(X_{1}^{\prime}=X_{1}^{\prime \prime}, X_{2}^{\prime}>X_{2}^{\prime \prime}\right) \\
& +P\left(X_{1}^{\prime}>X_{1}^{\prime \prime}, X_{2}^{\prime}=X_{2}^{\prime \prime}\right) \\
& -P\left(X_{1}^{\prime}>X_{1}^{\prime \prime}, X_{2}^{\prime}<X_{2}^{\prime \prime}\right)-P\left(X_{1}^{\prime}<X_{1}^{\prime \prime}, X_{2}^{\prime}>X_{2}^{\prime \prime}\right)
\end{aligned}
$$

Fixando os valores $x_{1}^{\prime \prime}$ e $x_{2}^{\prime \prime}$, assumimos a notação discreta, $P(x, y), p(x, y)$, f.d.a. e f.d.p. conjunta entre $X_{1}$ e $X_{2} . F_{i}$ e $f_{i}$, f.d.a. e f.d.p. marginal, para a variável $X_{i}, i=1,2$.

$$
P\left(X_{1}^{\prime}>x_{1}^{\prime \prime}, X_{2}^{\prime}=x_{2}^{\prime \prime}\right)=\sum_{j=x_{1}^{\prime \prime}+1}^{+\infty} p\left(j, x_{2}^{\prime \prime}\right),
$$

logo,

$$
\begin{aligned}
P\left(X_{1}^{\prime}>X_{1}^{\prime \prime}, X_{2}^{\prime}=X_{2}^{\prime \prime}\right) & =\sum_{x_{1}^{\prime \prime}} \sum_{x_{2}^{\prime \prime}} p\left(x_{1}^{\prime \prime}, x_{2}^{\prime \prime}\right) \sum_{j=x_{1}^{\prime \prime}+1}^{+\infty} p\left(j, x_{2}^{\prime \prime}\right) \\
P\left(X_{1}^{\prime}=X_{1}^{\prime \prime}, X_{2}^{\prime}>X_{2}^{\prime \prime}\right) & =\sum_{x_{1}^{\prime \prime}} \sum_{x_{2}^{\prime \prime}} p\left(x_{1}^{\prime \prime}, x_{2}^{\prime \prime}\right) \sum_{i=x_{2}^{\prime \prime}+1}^{+\infty} p\left(x_{1}^{\prime \prime}, i\right) \\
P\left(X_{1}^{\prime}>X_{1}^{\prime \prime}, X_{2}^{\prime}<X_{2}^{\prime \prime}\right) & =\sum_{x_{2}^{\prime \prime}} \sum_{x_{1}^{\prime \prime}} p\left(x_{1}^{\prime \prime}, x_{2}^{\prime \prime}\right)\left\{\sum_{j>x_{1}^{\prime \prime}} \sum_{i<x_{2}^{\prime \prime}} p(j, i)\right\} \\
P\left(X_{1}^{\prime}<X_{1}^{\prime \prime}, X_{2}^{\prime}>X_{2}^{\prime \prime}\right) & =\sum_{x_{2}^{\prime \prime}} \sum_{x_{1}^{\prime \prime}} p\left(x_{1}^{\prime \prime}, x_{2}^{\prime \prime}\right)\left\{\sum_{j<x_{1}^{\prime \prime}} \sum_{i>x_{2}^{\prime \prime}} p(j, i)\right\}
\end{aligned}
$$

Assumimos que os domínios $D_{1}$ e $D_{2}$ das variáveis $X_{1}$ e $X_{2}$, respectivamente. são seqüências consecutivas de reais. Com estas considerações, a medida $\tau$ fica 


$$
\begin{aligned}
\tau_{X_{1}, X_{2}}= & 2 \sum_{i_{1} \in D_{1}} \sum_{i_{2} \in D_{2}} P\left(i_{1}, i_{2}\right) p\left(i_{1}, i_{2}\right)-\sum_{i_{1} \in D_{1}} F_{1}\left(i_{1}\right) f_{1}\left(i_{1}\right)-\sum_{i_{2} \in D_{2}} F_{2}\left(i_{2}\right) f_{2}\left(i_{2}\right) \\
& +\sum_{i_{2} \in D_{2}} \sum_{i_{1} \in D_{1}} p\left(i_{1}, i_{2}\right)\left\{\sum_{\substack{j \in D_{1}, j>i_{1}}} p\left(j, i_{2}\right)\right\} \\
& +\sum_{i_{2} \in D_{2}} \sum_{i_{1} \in D_{1}} p\left(i_{1}, i_{2}\right)\left\{\sum_{\substack{i \in D_{2}, i>i_{2}}} p\left(i_{1}, i\right)\right\}+1 \\
& -\sum_{i_{2} \in D_{2}} \sum_{i_{1} \in D_{1}} p\left(i_{1}, i_{2}\right)\left\{\sum_{\substack{j \in D_{1}, j>i_{1}}} \quad i \in D_{2},\right. \\
& -\sum_{i_{2} \in D_{2}} \sum_{i_{1} \in D_{1}} p\left(i_{1}, i_{2}\right)\left\{\begin{array}{l}
j<i_{2} \\
j \in D_{1}, \quad i \in D_{2}, \\
j<i_{1} \quad i>i_{2}
\end{array}\right.
\end{aligned}
$$

Definamos entre as variáveis $X_{1}$ e $X_{2}$ a seguinte medida de associação, como fizemos para variáveis contínuas. Sejam $i_{j}, i_{j}^{\prime} \in D_{j}, i_{j} \leq i_{j}^{\prime}, j=1,2$.

$$
\begin{aligned}
T_{X_{1}, X_{2}}:=\sum_{i_{2}^{\prime} \in D_{2}} \sum_{i_{1}^{\prime} \in D_{1}} \sum_{\substack{i_{2} \in D_{2}, i_{2} \leq i_{2}^{\prime}}} \sum_{\substack{i_{1} \in D_{1} \\
i_{1} \leq i_{1}^{\prime}}}\left\{p\left(i_{1}^{\prime}, i_{2}^{\prime}\right) p\left(i_{1}, i_{2}\right)-p\left(i_{1}, i_{2}^{\prime}\right) p\left(i_{1}^{\prime}, i_{2}\right)\right\} \\
T_{X_{1}, X_{2}}=\sum_{i_{2} \in D_{2}} \sum_{i_{1} \in D_{1}} P\left(i_{1}, i_{2}\right) p\left(i_{1}, i_{2}\right)-\sum_{i_{2} \in D_{2}} \sum_{i_{1} \in D_{1}} P\left(X_{1} \leq i_{1}, X_{2}=i_{2}\right) P\left(X_{1}=i_{1}, X_{2} \leq i_{2}\right)
\end{aligned}
$$

Finalmente,

$$
T_{X_{1}, X_{2}}=\sum_{i_{2} \in D_{2}} \sum_{i_{1} \in D_{1}} P\left(i_{1}, i_{2}\right) p\left(i_{1}, i_{2}\right)-\sum_{i_{2} \in D_{2}} \sum_{i_{1} \in D_{1}}\left\{\sum_{\substack{j \in D_{1} \\ j \leq i_{1}}} p\left(j, i_{2}\right)\right\}\left\{\sum_{\substack{i \in D_{2} \\ i \leq i_{2}}} p\left(i_{1}, i\right)\right\}
$$

\footnotetext{
${ }^{0}$ As fórmulas aqui apresentadas, $\tau, T$ são implementadas num programa-vide Apêndice.
} 
Nota 1:

Notemos que para cada termo da medida $\tau$ podemos achar uma expressão equivalente em função do acoplamento associado $C_{1,2}$ ou suas marginais acumuladas $C_{1}$ e $C_{2}$. Por exemplo,

$$
\sum_{i_{2} \in D_{2}} \sum_{i_{1} \in D_{1}} P\left(i_{1}, i_{2}\right) p\left(i_{1}, i_{2}\right)=\sum_{i_{2} \in D_{2}} \sum_{i_{1} \in D_{1}} C_{1,2}\left(F_{1}\left(i_{1}\right), F_{2}\left(i_{2}\right)\right) c_{1,2}\left(u_{1}, u_{2}\right) f_{1}\left(i_{1}\right) f_{2}\left(i_{2}\right),
$$

onde $u_{j} \in\left[F_{j}\left(i_{j}-1\right), F_{j}\left(i_{j}\right)\right], j=1,2$, e $C_{1,2}$ é um acoplamento associado à f.d.a.P.

Considerando. $u_{j}=F_{j}\left(i_{j}\right), \Delta u_{j}=f_{j}\left(i_{j}\right) j=1,2$, temos,

$$
\sum_{i_{2} \in D_{2}} \sum_{i_{1} \in D_{1}} P\left(i_{1}, i_{2}\right) p\left(i_{1}, i_{2}\right)=\sum_{u_{2}} \sum_{u_{1}} C_{1,2}\left(u_{1}, u_{2}\right) c_{1,2}\left(u_{1}, u_{2}\right) \Delta u_{1} \Delta u_{2}
$$

Nota 2:

Notemos que, quando $F_{1}$ e $F_{2}$ são contínuas, retornamos a 3.2 .6 e a 3.2 .8 [Nelsen (1992)]. Assim, para o caso discreto, em geral, $\tau_{X_{1}, X_{2}}$ pode não constituir uma medida da associação $T P_{2}\left(R R_{2}\right)$. 


\subsubsection{Exemplo}

Exemplo do uso da quantidade $T$ como medida de associação.

Consideremos quatro variáveis aleatórias $Y, X_{1}, X_{2}, X_{3}$ com distribuição multinomial de parâmetros $n=1$ e $\left(p, p_{1}, p_{2}, p_{3}\right)$, onde as variáveis $X_{i}, i=1,2,3$ representam resultados de diferentes análises clínicas feitas em um paciente. A variável $Y$ representa o diagnóstico do médico, em relação aos resultados $X_{1}, X_{2}, X_{3}$. Assim,

$$
\begin{aligned}
P(Y & \left.=y, X_{1}=x_{1}, X_{2}=x_{2}, X_{3}=x_{3}\right) \\
& = \begin{cases}\frac{p^{y} p_{1}^{x_{1}} p_{2}^{x_{2}} p_{3}^{x_{3}}}{y ! x_{1} ! x_{2} ! x_{3} !} & \text { se } y+\sum_{i=1}^{3} x_{i}=1, \quad p+\sum_{i=1}^{3} p_{i}=1, p>0, p_{i}>0, \forall i \\
0 & \text { caso contrário }\end{cases}
\end{aligned}
$$

conseqüentemente,

$$
\begin{gathered}
Y \sim \operatorname{Bin}(1, p), X_{i} \sim \operatorname{Bin}\left(1, p_{i}\right) i=1,2,3 ; \\
P\left(Y=y, X_{i}=x_{i}\right)= \begin{cases}\frac{p^{y} p_{i}^{x_{i}}\left(1-p-p_{i}\right)^{1-y-x_{i}}}{\left(1-y-x_{i}\right) ! y ! x_{i} !} & \text { se } y+x_{i} \leq 1, \quad x_{i}, y \geq 0, \text { inteiros } \\
0 & \text { caso contrário }\end{cases} \\
P\left(X_{i}=x_{i}, X_{j}=x_{j}\right)=\left\{\begin{array}{ll}
\frac{p_{i}^{x_{i}} p_{j}^{x_{j}}\left(1-p_{i}-p_{j}\right)^{1-x_{i}-x_{j}}}{\left(1-x_{i}-x_{j}\right) ! x_{i} ! x_{j} !} & \text { se } x_{i}+x_{j} \leq 1, \quad x_{i}, x_{j} \geq 0, \text { inteiros } \\
0 & \text { caso contrário }
\end{array} ;\right. \\
P\left(Y=y, X_{i}=x_{i}, X_{j}=x_{j}\right) \\
= \begin{cases}\frac{p^{y} p_{i}^{x_{i}} p_{j}^{x_{j}}\left(1-p-p_{i}-p_{j}\right)^{1-y-x_{i}-x_{j}}}{y ! x_{i} ! x_{j} !\left(1-y-x_{i}-x_{j}\right) !} & \text { se } y+x_{i}+x_{j} \leq 1, y, x_{i}, x_{j} \geq 0, \text { inteiros } \\
0 & \text { caso contrário }\end{cases}
\end{gathered}
$$


Estudo do tipo de associação entre os pares de variáveis consideradas.

Como $\left(Y, X_{1}, X_{2}, X_{3}\right)$ tem distribuição multinomial de parâmetros 1 e $\left(p, p_{1}, p_{2}, p_{3}\right)$. então $\left(Y, X_{1}, X_{2}, X_{3}\right)$ é $R R_{2}$ em pares. Conseqüentemente, o vetor $\left(Y, X_{1}, X_{2}, X_{3}\right)$ tem suas variáveis $N A$ em pares.

Consideremos, agora, uma combinação linear entre as variáveis $X_{1}, X_{2}, X_{3}$,

$$
X_{4}:=a X_{1}+b X_{2}+c X_{3}, \quad 0<a \leq b \leq c
$$

Por exemplo, $X_{4}=X_{1}+2 X_{2}+4 X_{3}$. Temos,

$$
P\left(Y=y, X_{4}=x_{4}\right)=\sum_{x_{1}}^{\sum} \sum_{x_{2}}^{\sum} P\left(Y=y, X_{1}=x_{1}, X_{2}=x_{2}, X_{3}=\frac{x_{4}-a x_{1}-b x_{2}}{c}\right) .
$$

Mais especificamente,

$$
\begin{aligned}
& P\left(Y=0, X_{4}=a\right)=P\left(Y=0, X_{1}=1, X_{2}=0, X_{3}=0\right)=p_{1} \\
& P\left(Y=0, X_{4}=b\right)=P\left(Y=0, X_{1}=0, X_{2}=1, X_{3}=0\right)=p_{2} \\
& P\left(Y=0, X_{4}=c\right)=P\left(Y=0, X_{1}=0, X_{2}=0, X_{3}=1\right)=p_{3} \\
& P\left(Y=1, X_{4}=0\right)=P\left(Y=1, X_{1}=0, X_{2}=0, X_{3}=0\right)=p .
\end{aligned}
$$

Notemos que as quantidades $T_{Y, X_{i}}$ (médias das medidas locais da condição $T P_{2}\left(R R_{2}\right)$ ).

verificam $T_{Y, X_{4}} \leq T_{Y, X_{i}}, \quad i=1,2,3$.

$$
\begin{aligned}
& T_{Y, X_{i}}=-p p_{i}, \quad i=1,2,3, \\
& T_{Y, X_{4}}=-p\left\{p_{1}+p_{2}+p_{3}\right\} .
\end{aligned}
$$


Cabe destacar que neste exemplo não dá para extrair informação a partir das medidas $\tau_{Y, X_{i}}$, com relação ao tipo de associação. No entanto, é possível fazer isso a partir das médias das medidas locais da condição $T P_{2}\left(R R_{2}\right)$, ou seja a partir das quantidades $T_{Y, X_{i}}$, pois, $p_{i} \leq p_{1}+p_{2}+p_{3}, \forall i=1,2,3$, então $T_{Y, X_{4}} \leq T_{Y, X_{i}}, i=1,2,3$. Logo, as variáveis $Y$ e $X_{4}$ são mais negativamente associadas (desde o conceito de médias locais da condição $\left.T P_{2}\left(R R_{2}\right)\right)$ que $Y$ e $X_{i}, i=1,2,3$. Ou seja, a combinação de todas as $X_{i}, i=1,2,3$, potencializaram a associação negativa que existia entre as variáveis originais $X_{i}, i=1,2,3$ e a variável $Y$.

3.4.5 Exemplos: Em seguida apresentamos dois exemplos, fornecendo as medi$\operatorname{das} \tau$ e $T$.

Retomando as tabelas dos exemplos discretos

Exemplo a: $p(x, y)$

\begin{tabular}{|c|c|c|c|}
\hline \hline $\mathrm{Y} \backslash \mathrm{X}$ & 0 & 1 & $f_{2}(\cdot)$ \\
\hline 0 & $1 / 4$ & $1 / 8$ & $3 / 8$ \\
\hline 1 & $3 / 8$ & $1 / 4$ & $5 / 8$ \\
\hline$f_{1}(\cdot)$ & $5 / 8$ & $3 / 8$ & 1 \\
\hline \hline
\end{tabular}

$$
\tau_{X, Y}=0,81, T_{X, Y}=0,02 .
$$

Exemplo b: $p(x, y)$

\begin{tabular}{|c|c|c|c|c|}
\hline \hline $\mathrm{Y} \backslash \mathrm{X}$ & 0 & 1 & 2 & $f_{2}(\cdot)$ \\
\hline 0 & .15 & .19 & .15 & .49 \\
\hline 1 & .12 & .21 & .18 & .51 \\
\hline$f_{1}(\cdot)$ & .27 & .40 & .33 & 1 \\
\hline \hline
\end{tabular}

$$
\tau_{X, Y}=0,71, T_{X, Y}=0,02 .
$$




\section{LA VLELTA DE MARTIN FIERRO}

Viene uno como dormido Cuando vuelve del desierto Veré si a esplicarme aciento Entre gente tan bizarra. Y si al sentir la guitarra

De mi sueño me despierto.

Siento que mi pecho tiembla,

Que sé turba mi razón.

$Y$ de la viguiela al son

Imploro a la alma de un sabio

Que venga a mover mi labio

$Y$ alentar mi corazón.

Mucho tiene que contar

El que tuvo que sufrir.

Y empezaré por pedir

No duden de cuanto digo:

Pues debe creerse al testigo

Si no pagan por mentir.
Cantal el pueblero... y es puetat.

Canta el gaucho... y jay Jesús!

Lo miran como avestruz.

Su inorancia los asombra;

Mas siempre sirven las sombras Para distinguir la luz.

El campo es del inorante. El pueblo del hombre estruido Yo que en el campo he nacido

Digo que mis cantos son.

Paratos lunos - sonidos.

Y para otros - intención.

Yo he conocido cantores Que era un gusto el escuchar -

Mas no quieren opinar

$Y$ se divierten cantando;

Pero yo canto opinando

Que es mi modo de cantat.

Y el que me quiera enmendar

Mucho tiene que saber -

Tiene mucho que aprender

El que me sepa escuchar -

Tiene mucho que rumiar

El que me quiera entender.

\section{Capítulo 4 Análise Exaustiva da Medida $\tau$-Kendall}

- Seção 4.1: Máxima Associação Positiva (Negativa)

- Seção 4.2: Medida $\tau$-Kendall no Caso Discreto 


\subsection{Máxima Associação Positiva (Negativa)}

\section{Objetivo}

Estudar os valores extremos da medida de concordância $\tau$ de Kendall, quando ela representa uma medida de associação, i.e., marginais, acumuladas, univariada contínuas.

Estudo da associação positiva $\left(T P_{2}\right)$

A importância da seguinte proposição reside em caracterizar as relações entre variáveis aleatórias com a ajuda dos valores assumidos pela medida $\tau$, e suas implicações.

A partir de outro ponto de vista, a seguinte proposição é muito mais geral do que parece, se lembrarmos que sempre existe um acoplamento associado e que, por definição, ele possui marginais uniformes. 


\subsubsection{Proposição*}

Suponhamos que as variáveis aleatórias $X_{1}$ e $X_{2}$ têm distribuição conjunta $F_{X_{1}, X_{2}}$ e marginais $U(0,1)$. Então, as seguintes condições são equivalentes,

(i) $\quad \tau_{X_{1}, X_{2}}=1$,

(ii) $P\left(X_{1}=X_{2}\right)=1$,

(iii) $\quad F_{X_{1}, X_{2}}\left(x_{1}, x_{2}\right)=\min \left\{x_{1}, x_{2}\right\}, x_{1}, x_{2} \in I=[0,1]$,

(iv) $\quad X_{2}=f\left(X_{1}\right)$ q.t.p. $\left[f_{X_{1}, X_{2}}\right]$ para alguma função $f$ não decrescente.

Prova de 4.1.1:

$(i) \Rightarrow(i i)$ :

propriedade: se $F_{X_{1}, X_{2}}$ é uma distribuição bivariada com marginais uniformes, então, a função de sobrevivência conjunta de $\left(X_{1}, X_{2}\right)$ tem a seguinte expressão

$$
P\left(X_{1}>x_{1}, X_{2}>x_{2}\right)=F_{X_{1}, X_{2}}\left(x_{1}, x_{2}\right)-x_{1}-x_{2}+1, \quad 0 \leq x_{1}, x_{2} \leq 1
$$

pois

$$
\begin{gathered}
P\left(X_{1}>x_{1}, X_{2}>x_{2}\right)=P\left(X_{1} \leq x_{1}, X_{2} \leq x_{2}\right)-P\left(X_{1} \leq x_{1}\right)-P\left(X_{2} \leq x_{2}\right)+1, \\
\forall x_{1}, x_{2}: 0 \leq x_{1}, x_{2} \leq 1 .
\end{gathered}
$$

Considerando que $\left(X_{1}, X_{2}\right)$ e $\left(X_{1}^{\prime}, X_{2}^{\prime}\right)$ são duas realizações independentes com igual função de distribuição acumulada $F_{X_{1}, X_{2}}$, 


$$
\begin{aligned}
1 & =\tau_{X_{1}, X_{2}} \\
& =P\left\{\left(X_{1}^{\prime}-X_{1}\right)\left(X_{2}^{\prime}-X_{2}\right) \geq 0\right\}-P\left\{\left(X_{1}^{\prime}-X_{1}\right)\left(X_{2}^{\prime}-X_{2}\right)<0\right\} \\
& =P\left\{\left(X_{1}^{\prime}-X_{1}\right)\left(X_{2}^{\prime}-X_{2}\right) \geq 0\right\}
\end{aligned}
$$

i.e.,

$$
1=\int_{-\infty}^{+\infty} \int_{-\infty}^{+\infty} P\left\{\left(X_{1}^{\prime}-X_{1}\right)\left(X_{2}^{\prime}-X_{2}\right) \geq 0 \mid X_{1}=x_{1}, X_{2}=x_{2}\right\} d F_{X_{1}, X_{2}}\left(x_{1}, x_{2}\right)
$$

A saber, condicionando sobre os possíveis valores de $\left(X_{1}, X_{2}\right)$, digamos, $\left(x_{1}, x_{2}\right)$, e usando a independência das observações $\left(X_{1}, X_{2}\right)$ e $\left(X_{1}^{\prime}, X_{2}^{\prime}\right)$, temos as seguintes igualdades q.t.p., integrando em ambos os membros na densidade conjunta.

$$
\begin{array}{rlr}
1= & P\left\{\left(X_{1}^{\prime}-x_{1}\right)\left(X_{2}^{\prime}-x_{2}\right) \geq 0\right\} & \\
= & P\left\{X_{1}^{\prime}>x_{1}, X_{2}^{\prime}>x_{2}\right\}+P\left\{X_{1}^{\prime}<x_{1}, X_{2}^{\prime}<x_{2}\right\}+ & \\
& P\left\{X_{1}^{\prime}=x_{1}, X_{2}^{\prime}>x_{2}\right\}+P\left\{X_{1}^{\prime}=x_{1}, X_{2}^{\prime}<x_{2}\right\}+ & \\
& P\left\{X_{1}^{\prime}>x_{1}, X_{2}^{\prime}=x_{2}\right\}+P\left\{X_{1}^{\prime}<x_{1}, X_{2}^{\prime}=x_{2}\right\} & {\left[f_{X_{1}, X_{2}}\right]} \\
= & P\left(X_{1}^{\prime}>x_{1}, X_{2}^{\prime}>x_{2}\right)+F_{X_{1}, X_{2}}\left(x_{1}, x_{2}\right),\left(F_{X_{1}, X_{2}} \text { contínua }\right) & \\
= & 2 F_{X_{1}, X_{2}}\left(x_{1}, x_{2}\right)-x_{1}-x_{2}+1 & {\left[f_{X_{1}, X_{2}}\right],}
\end{array}
$$

onde a última igualdade decorre da expressão da função de sobrevivência. Assim, $F_{X_{1}, X_{2}}\left(x_{1}, x_{2}\right)=\frac{x_{1}+x_{2}}{2}, \forall 0<x_{1}, x_{2}<1$, q.t.p.; e como

$$
F_{X_{1}, X_{2}}\left(x_{1}, x_{2}\right) \leq \min \left\{x_{1}, x_{2}\right\}
$$

(pois $F_{X_{1}, X_{2}}$ tem marginais uniformes, $\operatorname{Sklar}(1959)$ ) segue-se que $x_{1}=x_{2}$, q.t.p.. 
$($ ii $) \Rightarrow(i i i)$ :

$$
\begin{aligned}
F_{X_{1}, X_{2}}\left(x_{1}, x_{2}\right) & =P\left(X_{1} \leq x_{1}, X_{2} \leq x_{2}\right) \\
& =P\left(X_{1} \leq x_{1}, X_{2} \leq x_{2}, X_{1}=X_{2}\right) \\
& =P\left(X_{1} \leq \min \left\{x_{1}, x_{2}\right\}\right) \\
& =\min \left\{x_{1}, x_{2}\right\}
\end{aligned}
$$

onde, a segunda igualdade é conseqüência de $P\left(X_{1}=X_{2}\right)=1$, enquanto que a última decorre da hipótese (marginais $U(0,1)$ ).

$$
(i i i) \Rightarrow(i v) \text { : }
$$

Definição: seja $D$ subconjunto de $R^{2}$. Dizemos que $D$ é não decrescente, sempre que, para todo par de pontos $(x, y),(u, v)$ em $D, x<u \Rightarrow y \leq v$.

(iii) $\Rightarrow \exists D \subseteq R^{2}$ conjunto não decrescente, tal que $P\left(\left(X_{1}, X_{2}\right) \in D\right)=1$ [Mikusinski, Sherwood et al.(1991-1992)].

Consideremos

$$
f\left(x_{1}\right):=\frac{1}{2} \int_{-\infty}^{+\infty} I_{D}\left(x_{1}, x_{2}\right) f_{X_{2}}\left(x_{2}\right) d x_{2}
$$

onde

$$
I_{D}\left(x_{1}, x_{2}\right):=\left\{\begin{array}{ll}
1 & \text { se }\left(x_{1}, x_{2}\right) \in D \\
0 & \text { cc }
\end{array} .\right.
$$

Logo,

$$
E_{X_{1}, X_{2}}(f)=\int_{-\infty}^{+\infty} \int_{-\infty}^{+\infty} f\left(x_{1}\right) f_{X_{1}, X_{2}}\left(x_{1}, x_{2}\right) d x_{1} d x_{2}=\frac{1}{2}
$$


pois

$$
\begin{aligned}
E_{X_{1}, X_{2}}(f) & =\frac{1}{2} \int_{-\infty}^{+\infty} \int_{-\infty}^{+\infty}\left\{\int_{-\infty}^{+\infty} I_{D}\left(x_{1}, x_{2}\right) f_{X_{2}}\left(x_{2}\right) d x_{2}\right\} d x_{1} d x_{2} \\
& =\frac{1}{2} \int_{-\infty}^{+\infty}\left\{\int_{-\infty}^{+\infty} \int_{-\infty}^{+\infty} I_{D}\left(x_{1}, x_{2}\right) f_{X_{1}, X_{2}}\left(x_{1}, x_{2}\right) d x_{1} d x_{2}\right\} f_{X_{2}}\left(x_{2}\right) d x_{2} \\
& =\frac{1}{2} \int_{-\infty}^{+\infty} P\left\{\left(X_{1}, X_{2}\right) \in D\right\} f_{X_{2}}\left(x_{2}\right) d x_{2} \\
& =\frac{1}{2} \int_{-\infty}^{+\infty} f_{X_{2}}\left(x_{2}\right) d x_{2} \\
& =\frac{1}{2}
\end{aligned}
$$

por outro lado,

$$
\int_{-\infty}^{+\infty} \int_{-\infty}^{+\infty} x_{2} f_{X_{1}, X_{2}}\left(x_{1}, x_{2}\right) d x_{1} d x_{2}=\frac{1}{2}, \text { pois } X_{2} \text { é } U(0,1) .
$$

Finalmente,

$$
X_{2}=f\left(X_{1}\right) \text { q.t.p. }\left[f_{X_{1}, X_{2}}\right] \text {. }
$$

Resta verifica que $f$ é não decrescente.

Consideremos $x_{1}<x_{1}^{\prime}$,

como $D$ é não decrescente, dados $\left(x_{1}, x_{2}\right),\left(x_{1}^{\prime}, x_{2}^{\prime}\right)$ em $D$.

Então,

$$
I_{D}\left(x_{1}, x_{2}\right)=1=I_{D}\left(x_{1}^{\prime}, x_{2}^{\prime}\right)
$$

sempre que $x_{1}<x_{1}^{\prime}$ resulta $x_{2} \leq x_{2}^{\prime}$. Logo, 


$$
\begin{aligned}
f\left(x_{1}\right) & =\frac{1}{2} \int_{0}^{1} I_{D}\left(x_{1}, x_{2}\right) d x_{2} \\
& \leq \frac{1}{2} \int_{0}^{1} I_{D}\left(x_{1}^{\prime}, x_{2}^{\prime}\right) I_{\left(x_{2}, 1\right]}\left(x_{2}^{\prime}\right) d x_{2} \\
& =\frac{1}{2} \int_{0}^{1} I_{D}\left(x_{1}^{\prime}, x_{2}^{\prime}\right) I_{\left[0, x_{2}^{\prime}\right)}\left(x_{2}\right) d x_{2}^{\prime} \\
& \leq \frac{1}{2} \int_{0}^{1} I_{D}\left(x_{1}^{\prime}, x_{2}^{\prime}\right) d x_{2}^{\prime} \\
& =f\left(x_{1}^{\prime}\right) .
\end{aligned}
$$

$(i v) \Rightarrow(i):$

$$
\tau_{X_{1}, X_{2}}=2 \eta_{X_{1}, X_{2}}-1, \eta_{X_{1}, X_{2}}=P\left(\left(X_{1}-X_{1}^{\prime}\right)\left(X_{2}-X_{2}^{\prime}\right) \geq 0\right)
$$

onde $\left(X_{1}, X_{2}\right)$ e $\left(X_{1}^{\prime}, X_{2}^{\prime}\right)$ são realizações independentes com f.d.a. $F_{X_{1}, X_{2}}$.

Por outro lado,

$$
\begin{gathered}
1=P\left(X_{1} \geq x_{1}^{\prime}\right)+P\left(X_{1}<x_{1}^{\prime}\right) \forall x_{1}^{\prime} \in \text { Imagem de } X_{1} . \\
1=P\left(X_{1} \geq x_{1}^{\prime}, f\left(X_{1}\right) \geq f\left(x_{1}^{\prime}\right)\right)+P\left(X_{1} \geq x_{1}^{\prime}, f\left(X_{1}\right)<f\left(x_{1}^{\prime}\right)\right)+ \\
P\left(X_{1}<x_{1}^{\prime}, f\left(X_{1}\right)<f\left(x_{1}^{\prime}\right)\right)+P\left(X_{1}<x_{1}^{\prime}, f\left(X_{1}\right) \geq f\left(x_{1}^{\prime}\right)\right)\left[f_{X_{1}, X_{2}}\right] .
\end{gathered}
$$

Logo, como $f$ é não decrescente,

$$
1=P\left(X_{1} \geq x_{1}^{\prime}, X_{2} \geq x_{2}^{\prime}\right)+P\left(X_{1}<x_{1}^{\prime}, X_{2}<x_{2}^{\prime}\right)\left[f_{X_{1}, X_{2}}\right]
$$

onde $X_{2}=f\left(X_{1}\right)$ e $x_{2}^{\prime}=f\left(x_{1}^{\prime}\right)$

$$
\eta_{X_{1}, X_{2}}=1 \Rightarrow \tau_{X_{1}, X_{2}}=1
$$


Como conseqüência,

\subsubsection{Corolário *}

Sejam $X_{1}, X_{2}, X_{3}$ variáveis aleatórias, $\varphi: R^{2} \rightarrow R, X_{1}$ com distribuição $U(0,1)$ e $\varphi\left(X_{2}, X_{3}\right)$ variável aleatória de distribuição $U(0,1)$. Então, as seguintes condições são equivalentes

(i) $\tau_{X_{1}, \varphi\left(X_{2}, X_{3}\right)}=1$,

(ii) $P\left(X_{1}=\varphi\left(X_{2}, X_{3}\right)\right)=1$,

(iii) $\quad F_{X_{1}, \varphi\left(X_{2}, X_{3}\right)}\left(x_{1}, x\right)=\min \left\{F_{X_{1}}\left(x_{1}\right), F_{\varphi\left(X_{2}, X_{3}\right)}(x)\right\}, x_{1}, x \in I$,

(iv) $\varphi\left(X_{2}, X_{3}\right)=f\left(X_{1}\right)$ q.t.p. $\left[f_{X_{1}, \varphi\left(X_{2}, X_{3}\right)}\right]$ para alguma $f$ não decrescente.

Notemos que 4.1.2 ganha força quando temos em mente o resultado de De Finetti de 1953 (em seguida, enunciamos o teorema), pois a partir da hipótese: $X_{2}$ e $X_{3}$ possuindo distribuição conjunta contínua, podemos achar uma função $\varphi: R^{2} \rightarrow R$, tal que $\varphi\left(X_{2}, X_{3}\right)$ seja uma variável aleatória uniforme.

O seguinte Corolário fornece uma extensão de 4.1.1 natural, quando interpretamos tal proposição a partir da existência de um acoplamento associado. 


\subsubsection{Corolário*}

Suponhamos que as variáveis aleatórias $X_{1}$ e $X_{2}$ têm distribuição conjunta $F_{X_{1}, X_{2}}$ e marginais acumuladas contínuas $F_{X_{1}}$ e $F_{X_{2}}$ respectivamente. Então, as seguintes condições são equivalentes

(i) $\quad \tau_{X_{1}, X_{2}}=1$,

(ii) $\quad F_{X_{1}, X_{2}}\left(x_{1}, x_{2}\right)=\min \left\{F_{X_{1}}\left(x_{1}\right), F_{X_{2}}\left(x_{2}\right)\right\}, \forall x_{i} \in$ Imagem de $X_{i} i=1,2$,

(iii) $\quad F_{X_{2}}\left(X_{2}\right)=f \circ F_{X_{1}}\left(X_{1}\right)$ q.t.p. $\left[f_{X_{1}, X_{2}}\right]$ para alguma função $f$ não decrescente.

Notemos que (iii) fica substituída por

$$
X_{2}=f\left(X_{1}\right) \text { q.t.p. }\left[f_{X_{1}, X_{2}}\right]
$$

para alguma função $f$ não decrescente, quando $F_{X_{2}}$ for inversível.

Prova de 4.1.3:

$(i) \Rightarrow(i i)$ : pois $\tau$ é invariante perante transformações não decrescentes. Logo,

$$
1=\tau_{U_{1}, U_{2}} \text { onde } \quad U_{i}=F_{X_{i}}\left(X_{i}\right), i=1,2
$$

Nota: imediatamente, por 4.1 .1 temos, $1=P\left(U_{1}=U_{2}\right)$.

Consideremos $x_{i} \in$ Imagem de $X_{i}, i=1,2$. 


$$
\begin{aligned}
F_{X_{1}, X_{2}}\left(x_{1}, x_{2}\right) & =C_{X_{1}, X_{2}}\left(F_{X_{1}}\left(x_{1}\right), F_{X_{2}}\left(x_{2}\right)\right), C_{X_{1}, X_{2}} \text { acopl. associado a } F_{X_{1}, X_{2}} \\
& =F_{U_{1}, U_{2}}\left(u_{1}, u_{2}\right), u_{i}=F_{X_{i}}\left(x_{i}\right), i=1,2 \text {, por } 3.3 .9 \\
& =\min \left\{u_{1}, u_{2}\right\}, \text { por } 4.1 .1 \\
& =\min \left\{F_{X_{1}}\left(x_{1}\right), F_{X_{2}}\left(x_{2}\right)\right\}
\end{aligned}
$$

se $U_{i}=F_{X_{i}}\left(X_{i}\right), i=1,2 \Rightarrow$

$$
\begin{aligned}
F_{U_{1}, U_{2}}\left(u_{1}, u_{2}\right) & =C_{X_{1}, X_{2}}\left(u_{1}, u_{2}\right), \text { por } 3.3 .9 \\
& =C_{X_{1}, X_{2}}\left(F_{X_{1}}\left(x_{1}\right), F_{X_{2}}\left(x_{2}\right)\right), \exists x_{i}: F_{X_{i}}\left(x_{i}\right)=u_{i}, i=1,2 \\
& =F_{X_{1}, X_{2}}\left(x_{1}, x_{2}\right) \\
& =\min \left\{F_{X_{1}}\left(x_{1}\right), F_{X_{2}}\left(x_{2}\right)\right\} \\
& =\min \left\{u_{1}, u_{2}\right\}
\end{aligned}
$$

logo, por 4.1.1 temos que $\exists f$ função não decrescente, tal que

$$
U_{2}=f\left(U_{1}\right) \text { q.t.p. }\left[f_{U_{1}, U_{2}}\right] \text {. }
$$

Finalmente,

$$
F_{X_{2}}\left(X_{2}\right)=f \circ F_{X_{1}}\left(X_{1}\right) \text { q.t.p. }\left[f_{X_{1}, X_{2}}\right] \text {. }
$$


A seguir, enunciamos e referenciamos alguns resultados a serem usados logo.

Lembrar 3.3.5: Se $X_{1}$ possui função de distribuição $F_{X_{1}}$ contínua, então $F_{X_{1}}\left(X_{1}\right)$ é uma variável aleatória com distribuição uniforme $(0,1)$.

4.1.4 Teorema (Esteves (1997)): Sejam $X_{1}$ e $U$ variáveis aleatórias com função de distribuição contínua $F_{X_{1}}$ e $F_{U}$, respectivamente. Então, existe $\phi: R \rightarrow R$ tal que as variáveis aleatórias $\phi\left(X_{1}\right)$ e $\phi(U)$ possuem, ambas, distribuição uniforme em $(0,1)$.

4.1.5 Teorema (De Finetti (1953)): Sejam $X_{2}$ e $X_{3}$ com função de distribuição conjunta $F_{X_{2}, X_{3}}$ contínua. Então, existe $\varphi_{2,3}: R^{2} \rightarrow R$ tal que a variável aleatória $\varphi_{2,3}\left(X_{2}, X_{3}\right)$ possui distribuição uniforme em $(0,1)$.

Esteves(1997) menciona uma generalização do resultado anterior, a saber:

se $\underline{X}=\left(X_{1}, \cdots, X_{n}\right)$ e $\underline{Y}=\left(Y_{1}, \cdots, Y_{n}\right)$ são vetores aleatórios com funções de distribuição conjunta contínuas $F_{\underline{X}}$ e $F_{\underline{Y}}$, respectivamente, e $H$ uma função de distribuição unidimensional qualquer, então existe uma função real $\phi: R^{n} \rightarrow R$ tal que as variáveis aleatórias $\phi(\underline{X})$ e $\phi(\underline{Y})$ têm ambas função de distribuição $H$.

Como conseqüência dos resultados anteriores e de 4.1.2, temos: 


\subsubsection{Proposição*}

Sejam $X_{1}$ uma variável aleatória com função de distribuição contínua $F_{X_{1}}$ e $X_{2}, X_{3}$ duas variáveis aleatórias que possuem função de distribuição conjunta contínua $F_{X_{2}, X_{3}}$. Então,

(a) existem $\varphi_{1}: R \rightarrow R$ tal que $\varphi_{1}\left(X_{1}\right)$ é uma variável aleatória $U(0,1)$ e $\varphi_{2,3}$ : $R^{2} \rightarrow R$ tal que $\varphi_{2,3}\left(X_{2}, X_{3}\right)$ é variável aleatória $U(0,1)$;

(b) as seguintes condições são equivalentes:

$$
\begin{aligned}
& \left(b_{1}\right) \quad F_{U, V}(u, v)=\min \left\{F_{U}(u), F_{V}(v)\right\}, \\
& \left(b_{2}\right) \quad \tau_{U, V}=1,
\end{aligned}
$$

onde, $U:=\varphi\left(X_{1}\right), V:=\varphi_{2,3}\left(X_{2}, X_{3}\right)$

Comentário: O estudo de acoplamentos ou distribuições de marginais uniformes em $(0,1)$ representa uma poderosa ferramenta, isto é: todas as distribuições estão sendo contempladas através do seu acoplamento associado. Nesse ponto, se $X_{1}$ é a variável aleatória a predizer, $\left(X_{2}, X_{3}\right)$ é a base para a predição e estamos procurando o aporte que a base faz respeito da variável a predizer, podemos considerar $\varphi_{2,3}\left(X_{2}, X_{3}\right)$ como um resumo do aporte feito pela base de predição e analisar a estutura de associação existente entre $X_{1}$ e a nova variável uniforme em $(0,1)$, ou como é proposto na Proposição anterior estudar a estrutura de associação entre $\varphi_{1}\left(X_{1}\right)$ e $\varphi_{2,3}\left(X_{2}, X_{3}\right)$. 


\subsubsection{Corolário*}

Na hipótese da proposição anterior, as seguintes condições são equivalentes

$$
\begin{aligned}
& \text { (i) } \quad F_{W, V}(w, v)=\min \left\{F_{W}(w), F_{V}(v)\right\}, \\
& (i i) \quad \tau_{W, V}=\tau_{X_{1}, V}=1 .
\end{aligned}
$$

onde, $W:=F_{X_{1}}\left(X_{1}\right), V:=\varphi_{2,3}\left(X_{2}, X_{3}\right)$.

Comentário: O resultado anterior tenta simplificar o tratamento da variável $X_{1} \mathrm{em}$ relação com a base de predição. Pelas dificuldades topológicas presentadas pela função $\varphi_{1}$ - vide Esteves (1997) - é proposto o estudo da estrutura de associação entre $X_{1}$ e a base de predição $\left(X_{2}, X_{3}\right)$ através do estudo das transformadas $F_{X_{1}}\left(X_{1}\right)$ e $\varphi_{2,3}\left(X_{2}, X_{3}\right)$, aproveitando as bondades da medida $\tau$ de Kendall em relação as transformações monótonas não decrescentes.

Provas de 4.1.6 e de 4.1.7:

Notemos que $W$ é uma variável aleatória $U(0,1)$, por 3.3.5, enquanto que $V:=$ $\varphi_{2,3}\left(X_{2}, X_{3}\right)$ é uma variável aleatória $U(0,1)$, por 4.1 .5 
A primeira parte de (a) de 4.1.6 é conseqüência de 3.3 .5 ou de 4.1.4, enquanto que a segunda parte, do mesmo tópico, decorre de 4.1 .5 (De Finetti (1953)). (b) de 4.1.6 resulta a partir de 4.1 .2 e da parte (a) prévia.

O tópico 4.1.7 apresenta uma variante de 4.1.6, considerando a transformação uniforme de $X_{1}, W=F_{X_{1}}\left(X_{1}\right)$, invocada por 3.3.5. A igualdade presente em (ii) decorre da invariância de $\tau$ perante transformações não decrescentes - 3.1.2. 
Estudo da associação negativa $\left(R R_{2}\right)$

\subsubsection{Proposição *}

Suponhamos que as variáveis aleatórias $X_{1}$ e $X_{2}$ têm distribuição acumulada conjunta $F_{X_{1}, X_{2}}$ e distribuições marginais $U(0,1)$. Então, as seguintes condições são equivalentes

$$
\begin{array}{ll}
\text { (i) } & \tau_{X_{1}, X_{2}}=-1, \\
\text { (ii) } & P\left(X_{2}=1-X_{1}\right)=1 \\
(\text { iii }) & F_{X_{1}, X_{2}}\left(x_{1}, x_{2}\right)=\max \left\{0, x_{1}+x_{2}-1\right\}, x_{1}, x_{2} \in I=[0,1] \\
\text { (iv) } & X_{2}=f\left(X_{1}\right) \text { q.t.p. }\left[f_{X_{1}, X_{2}}\right] \text { para alguma } f \text { não crescente. }
\end{array}
$$

Prova de 4.1.8:

$(i) \Rightarrow(i i)$ : análoga à prova feita em 4.1.1.

$$
\begin{aligned}
& (\text { ii }) \Rightarrow(\text { iii }) \text { : } \\
& \begin{aligned}
P\left(X_{1} \leq x_{1}, X_{2} \leq x_{2}\right) & =P\left(X_{1} \leq x_{1}, X_{2} \leq x_{2}, X_{2}=1-X_{1}\right) \\
& =P\left(1-x_{2} \leq X_{1} \leq x_{1}\right) \\
& =\left\{\begin{array}{cc}
x_{1}-\left[1-x_{2}\right] & \text { se } 0 \leq 1-x_{2}<x_{1} \leq 1 \\
0 & \text { caso contrário }
\end{array}\right. \\
& =\max \left\{0, x_{1}+x_{2}-1\right\} .
\end{aligned}
\end{aligned}
$$

$(i i i) \Rightarrow(i v)$ :

(iii) $\Rightarrow \exists S \subseteq R^{2}$, subconjunto não crescente, i.e., para todo par de pontos 
$(x, y),(u, v) \in S, x<u \Rightarrow v \leq y$.

Com argumentos equivalentes aos utilizados em 4.1.1 verifica-se (iv) [Mikusinski, Sherwood (1991)].

$$
\begin{aligned}
(i v) & \Rightarrow(i): \\
\tau_{X_{1}, X_{2}} & =P\left\{\left(X_{1}^{\prime}-X_{1}\right)\left(f\left(X_{1}^{\prime}\right)-f\left(X_{1}\right)\right) \geq 0\right\}-P\left\{\left(X_{1}^{\prime}-X_{1}\right)\left(f\left(X_{1}^{\prime}\right)-f\left(X_{1}\right)\right)<0\right\} \\
& =-P\left\{\left(X_{1}^{\prime}-X_{1}\right)\left(f\left(X_{1}^{\prime}\right)-f\left(X_{1}\right)\right)<0\right\} \\
& =-1,
\end{aligned}
$$

pois, para cada possível valor de $X_{1}=x_{1}, P\left\{\left(X_{1}^{\prime}-x_{1}\right)\left(f\left(X_{1}^{\prime}\right)-f\left(x_{1}\right)\right) \geq 0\right\}=0$ já que $f$ é uma função não crescente. Deste modo para cada $X_{1}=x_{1}$ só pode acontecer

$$
\left\{X_{1}^{\prime}<x_{1}, f\left(X_{1}^{\prime}\right)>f\left(x_{1}\right)\right\} \text { ou }\left\{X_{1}^{\prime}>x_{1}, f\left(X_{1}^{\prime}\right)<f\left(x_{1}\right)\right\}
$$

Logo, resulta $(i)$.

\subsubsection{Corolário*}

Sejam $X_{1}, X_{2}, X_{3}$ variáveis aleatórias, $\varphi: R^{2} \rightarrow R, X_{1}$ com distribuição $U(0,1)$ e $\varphi\left(X_{2}, X_{3}\right)$ variável aleatória $U(0,1)$. Então, as seguintes condições são equivalentes

(i) $\tau_{X_{1}, \varphi\left(X_{2}, X_{3}\right)}=-1$,

(ii) $\quad P\left(\varphi\left(X_{2}, X_{3}\right)=1-X_{1}\right)=1$,

(iii) $\quad F_{X_{1}, \varphi\left(X_{2}, X_{3}\right)}\left(x_{1}, x\right)=\max \left\{0, F_{X_{1}}\left(x_{1}\right)+F_{\varphi\left(X_{2}, X_{3}\right)}(x)-1\right\}, x_{1}, x \in I$,

(iv) $\varphi\left(X_{2}, X_{3}\right)=f\left(X_{1}\right)$ q.t.p. $\left[f_{X_{1}, \varphi\left(X_{2}, X_{3}\right)}\right] f$ não crescente 


\subsubsection{Proposição*}

Sejam $X_{1}$ uma variável aleatória com função de distribuição contínua $F_{X_{1}}$ e $X_{2}, X_{3}$ duas variáveis aleatórias que possuem função de distribuição conjunta contínua $F_{X_{2}, X_{3}}$. Então, as seguintes condições são equivalentes

$$
\begin{array}{ll}
\left(b_{11}\right) & F_{U, V}(u, v)=\max \left\{0, F_{U}(u)+F_{V}(v)-1\right\} \\
\left(b_{22}\right) & \tau_{U, V}=-1
\end{array}
$$

onde, $U=\varphi_{1}\left(X_{1}\right), V=\varphi_{2,3}\left(X_{2}, X_{3}\right), \varphi_{1}$ e $\varphi_{2,3}$ são como em 4.1.6.

\subsubsection{Corolário*}

$\mathrm{Na}$ hipótese da proposição anterior, as seguintes condições são equivalentes

$$
\begin{aligned}
& \text { (i) } \quad F_{W, V}(w, v)=\max \left\{0, F_{W}(w)+F_{V}(v)-1\right\}, \\
& (\text { ii }) \quad-1=\tau_{W, V}=\tau_{X_{1}, V},
\end{aligned}
$$

onde, $W=F_{X_{1}}\left(X_{1}\right), V=\varphi_{2,3}\left(X_{2}, X_{3}\right)$.

Nos casos extremos, os conceitos de acoplamento extremo maximizante $\min \left\{x_{1}, x_{2}\right\}$ e medida $\tau$ máxima: $\tau_{X_{1}, X_{2}}=1$, é interpretada como concordância entre as variáveis envolvidas. Para o acoplamento extremo mínimo, $\max \left\{0, x_{1}+x_{2}-1\right\}$ e a medida $\tau$ mínima: $\tau_{X_{1}, X_{2}}=-1$; as variáveis envolvidas desenvolvem um comportamento discordante. 


\subsubsection{A homotopia como conexão contínua entre ditribuições acumuladas}

É conhecido que cada vez que o par $\left(U_{1}, U_{2}\right)$ tem f.d.a. absolutamente contínua $F_{U_{1}, U_{2}}$ é possível definir $\left(T_{1}, T_{2}\right):=\Psi_{F_{U_{1}, U_{2}}}\left(U_{1}, U_{2}\right)$ onde,

$$
\Psi_{F_{U_{1}, U_{2}}}: R^{2} \rightarrow[0,1]^{2}, \quad \Psi_{F_{U_{1}, U_{2}}}\left(u_{1}, u_{2}\right)=\left(F_{U_{1}}\left(u_{1}\right), F_{U_{2} \mid u_{1}}\left(u_{2}\right)\right)
$$

para todo $\left(u_{1}, u_{2}\right)$ no suporte de $\left(U_{1}, U_{2}\right)$.

Logo, $T_{1}$ e $T_{2}$ são variáveis aleatórias independentes e uniformes em $(0,1)$ [H.Li; M. Scarsini; M. Shaked (1996)pp.23].

$F_{T_{1}, T_{2}}\left(t_{1}, t_{2}\right)=t_{1} t_{2}$. Por tanto, sua distribuição constitui um acoplamento. Isso significa que na passagem do par $\left(U_{1}, U_{2}\right)$ para o par $\left(T_{1}, T_{2}\right)$ é anulada toda a dependência existente.

Com uma ideia semelhante estamos procurando "potencializar a associação" existente entre $U_{1}$ e $U_{2}$.

Pensemos agora na situação extrema, isto é, dadas duas variáveis aleatórias $X_{1}$ e $X_{2}$ com função de distribuição acumulada $F_{X_{1}, X_{2}}$ de marginais uniformes em $(0,1), F_{X_{1}, X_{2}}$ é identicamente igual ao acoplamento máximo ou limitante superior de Fréchet se, e somente se, $F_{X_{1}, X_{2}}$ está concentrada numa curva não decrescente.

Resenha (P. Mikusinski; H. Sherwood e M.D. Taylor (1991-92) do Real Analysis Exchange, vol 17, "The Fréchet Bounds Revisited",pp.759-764]. 


\subsubsection{Aplicações Homotópicas}

\section{Premissa}

Verificar se é possível levar um par de variáveis aleatórias com distribuição conjunta de marginais uniformes, até um outro par, cuja f.d.a. seja o limitante superior da Classe de Fréchet.

Se as distribuições marginais acumuladas são $F_{X_{i}}, i=1, \cdots, n$, a Classe de Fréchet de $\left\{F_{X_{i}}\right\}_{i=1}^{n}$ é constituída pelas distribuições acumuladas conjuntas de marginais univariadas $F_{X_{i}}, i=1, \cdots, n$.

O limitante inferior: $\max \left\{0, \sum_{i=1}^{n} F_{X_{i}}\left(x_{i}\right)-(n-1)\right\}$ assim como o limitante superior: $\min _{1 \leq i \leq n}\left\{F_{X_{i}}\left(x_{i}\right)\right\}$ da Classe de Fréchet, são distribuições acumuladas conjuntas, elementos da Classe de Fréchet definida pela seqüência de marginais $\left\{F_{X_{i}}\right\}_{i=1}^{n}$ que no caso de $n=2$ representam estruturas de máxima associação negativa e positiva respectivamente.

Sejam $E_{1}$ e $E_{2}$ espaços topológicos, $I=[0,1]$. Duas aplicações contínuas $f, g$ : $E_{1} \rightarrow E_{2}$ dizem-se homotópicas, quando existe uma aplicação contínua $H: E_{1} \times I \rightarrow$ $E_{2}$ (chamada uma homotopia entre $f$ e $g$ ) tal que, para cada $x_{1} \in E_{1}, H\left(x_{1}, 0\right)=$ $f\left(x_{1}\right)$ e $H\left(x_{1}, 1\right)=g\left(x_{1}\right)$. 
Se $E_{1}$ for um espaço de Hausdorff localmente compacto, $f$ e $g$ são homotópicas se, e somemente se, pertencem à mesma componente conexa por caminhos no espaço $C_{c a}\left(E_{1} ; E_{2}\right)$. Tais componentes chamam-se as classes de homotopia das aplicações com domínio em $E_{1}$ e imagem em $E_{2}$.

Sendo $C\left(E_{1} ; E_{2}\right)$ o conjunto das aplicações contínuas de $E_{1}$ em $E_{2}$. A topologia em $C\left(E_{1} ; E_{2}\right)$ gerada pelos conjuntos $A(K, V):=\left\{f \in C\left(E_{1} ; E_{2}\right): f(K) \subseteq V\right\}$, onde $K$ percorre os subconjuntos compactos de $E_{1}$ e $V$ os abertos de $E_{2}$, chama-se a topologia compacto-aberta, e o espaço topológico correspondente é indicado por $C_{c a}\left(E_{1} ; E_{2}\right)$.

Considerando a seguir $R^{2}$ com a topologia usual, suponhamos que dispomos de uma f.d.a. contínua, $F_{U_{1}, U_{2}}$ que é a densidade acumulada conjunta das variáveis $U_{1}$ e $U_{2}$ cujas distribuições univariadas são $U(0,1)$. Evidentemente $F_{U_{1}, U_{2}}$ constitui um acoplamento. Consideremos o acoplamento máximo: $\min \left\{u_{1}, u_{2}\right\}$. Então, é possível enlaçar ambas as distribuições com uma homotopia

$$
\begin{aligned}
H & : R^{2} \times[0,1] \rightarrow[0,1], \text { tal que } \\
H\left(\left(u_{1}, u_{2}\right), 0\right) & =F_{U_{1}, U_{2}}\left(u_{1}, u_{2}\right), \\
H\left(\left(u_{1}, u_{2}\right), 1\right) & =\min \left\{u_{1}, u_{2}\right\} .
\end{aligned}
$$

As condições de contorno das funções a enlaçar, são as seguintes:

$$
\begin{aligned}
& F_{U_{1}, U_{2}}\left(u_{1}, 0\right)=\min \left\{u_{1}, 0\right\}=0, \forall u_{1} \in[0,1] \\
& F_{U_{1}, U_{2}}\left(u_{1}, 1\right)=\min \left\{u_{1}, 1\right\}=u_{1}, \forall u_{1} \in[0,1] \\
& F_{U_{1}, U_{2}}\left(0, u_{2}\right)=\min \left\{0, u_{2}\right\}=0, \forall u_{2} \in[0,1] \\
& F_{U_{1}, U_{2}}\left(1, u_{2}\right)=\min \left\{1, u_{2}\right\}=u_{2}, \forall u_{2} \in[0,1] .
\end{aligned}
$$


Consideremos a aplicação

$H\left(\left(u_{1}, u_{2}\right), t\right):=(1-t) F_{U_{1}, U_{2}}\left(u_{1}, u_{2}\right)+\operatorname{tmin}\left\{u_{1}, u_{2}\right\}, 0 \leq t \leq 1, u_{i} \in[0,1], i=1,2$.

A transformação anterior verifica

$$
\begin{gathered}
H\left(\left(u_{1}, u_{2}\right), 0\right)=F_{U_{1}, U_{2}}\left(u_{1}, u_{2}\right), \\
H\left(\left(u_{1}, u_{2}\right), 1\right)=\min \left\{u_{1}, u_{2}\right\}, \\
\forall u_{i} \in[0,1], i=1,2 . \\
H\left(\left(u_{1}, 1\right), t\right)=(1-t) u_{1}+t u_{1}=u_{1}, \\
H\left(\left(u_{1}, 0\right), t\right)=0, \\
\forall u_{1} \in[0,1], \forall t \in[0,1] . \\
H\left(\left(1, u_{2}\right), t\right)=(1-t) u_{2}+t u_{2}=u_{2}, \\
H\left(\left(0, u_{2}\right), t\right)=0,
\end{gathered}
$$

$$
\forall u_{2} \in[0,1], \forall t \in[0,1]
$$

Por outro lado, $H$ é contínua em $\left(u_{1}, u_{2}\right)$, pois $F_{U_{1}, U_{2}}$ e o $\min \left\{u_{1}, u_{2}\right\}$ são contínuas, $\forall u_{i} \in[0,1], i=1,2$. $H$ é contínua em $t$, por ser transformação linear.

Assim, vê-se que $H$ contitui uma homotopia linear que respeita as condições de contorno. 
A existência da homotopia traduz-se na possibilidade de deformar um caminho $F_{U_{1}, U_{2}}$ continuamente. Assim, $H$ deforma $F_{U_{1}, U_{2}}\left(u_{1}, u_{2}\right)$ em $\min \left\{u_{1}, u_{2}\right\}$ fazendo, para cada $\left(u_{1}, u_{2}\right) \in[0,1]^{2}$, o ponto $F_{U_{1}, U_{2}}\left(u_{1}, u_{2}\right)$ deslizar no tempo unitário, com velocidade constante. ao longo do segmento da reta $\left[F_{U_{1}, U_{2}}\left(u_{1}, u_{2}\right), \min \left\{u_{1}, u_{2}\right\}\right]$. Uma tal homotopia diz-se "linear" e os caminhos $F_{U_{1}, U_{2}}(\cdot, \cdot)$ e $\min \{\cdot, \cdot\}$ dizem-se, então, linearmente homotópicos.

4.1.14 Proposição* : Para cada tempo t. fixo, $H((\cdot, \cdot), t)$ é f.d.a.

Prova de 4.1.14:

basta notar que $F_{U_{1}, U_{2}}\left(u_{1}, u_{2}\right) \leq \min \left\{u_{1}, u_{2}\right\}, \forall u_{i} \in[0,1]$, para concluir que $H((\cdot, \cdot), t)$ associa probabilidade não nula aos quadrados em $R^{2}$, do tipo $(a, b] \times(c, d]$.

4.1.15 Exemplo: Um exemplo desta homotopia é apresentado em [Joe(1997)pp.148] onde

$$
C(u, v, t):=\operatorname{tmin}\{u, v\}+(1-t) u v, \forall t \in[0,1]
$$

Nesse caso, a homotopia transforma continuamente o caminho uv (acoplamento de independência) no caminho $\min \{u, v\}$ (acoplamento de máxima associação positiva). 
Medida bivariada $\tau$ de Kendall e comparação entre variáveis.

Vamos considerar um vetor tri-variado, para estudar as possibilidades da otimização da associação, i.e., sob o seguinte contexto: sejam $\left(X_{1}, X_{2}, X_{3}\right)$ variáveis aleatórias com f.d.p. $f_{X_{1}, X_{2}, X_{3}}$

Critérios de comparação

4.1.16 Definição Se $\left(X_{1}, X_{i}\right)$ é um vetor aleatório com f.d.p. conjunta $f_{X_{1}, X_{2}} T P_{2}$ para $i=2,3$. Dizemos que $\left(X_{1}, X_{2}\right)$ são localmente mais positivamente associadas $\left(T P_{2}\right)$ que $\left(X_{1}, X_{3}\right)$ se

$$
\begin{gathered}
f_{X_{1}, X_{2}}\left(x^{\prime}, y^{\prime}\right) f_{X_{1}, X_{2}}(x, y)-f_{X_{1}, X_{2}}\left(x^{\prime}, y\right) f_{X_{1}, X_{2}}\left(x, y^{\prime}\right) \\
\geq f_{X_{1}, X_{3}}\left(x^{\prime}, y^{\prime}\right) f_{X_{1}, X_{3}}(x, y)-f_{X_{1}, X_{3}}\left(x^{\prime}, y\right) f_{X_{1}, X_{3}}\left(x, y^{\prime}\right) \\
\forall\left(x^{\prime}, x, y^{\prime}, y\right) \text { tal que } x^{\prime} \geq x, y^{\prime} \geq y .
\end{gathered}
$$

4.1.17 Definição Se $\left(X_{1}, X_{i}\right)$ tem f.d.p. $f_{X_{1}, X_{i}} T P_{2}$ para $i=1$, 2. Dizemos que $\left(X_{1}, X_{2}\right)$ são mais positivamente associadas $\left(T P_{2}\right)$ que $\left(X_{1}, X_{3}\right)$ se

$$
\tau_{X_{1}, X_{2}} \geq \tau_{X_{1}, X_{3}}
$$


4.1.18 Proposição*: Sejam $X_{1}$ e $X_{i}$ variáveis aleatórias com f.d.p. conjunta $T P_{2}$. Se $\left(X_{1}, X_{2}\right)$ forem localmente mais positivamente associadas $\left(T P_{2}\right)$ que $\left(X_{1}, X_{3}\right)$, então

$$
\begin{gathered}
P\left(X_{2}>y \mid X_{1}=x^{\prime}\right)-P\left(X_{2}>y \mid X_{1}=x\right) \geq P\left(X_{3}>y \mid X_{1}=x^{\prime}\right)-P\left(X_{3}>y \mid X_{1}=x\right), \\
\forall x \leq x^{\prime} .
\end{gathered}
$$

Prova de 4.1.18: previamente devemos notar que, para $y, x, x^{\prime}$ arbitrários

$$
P\left(X_{i}>y \mid X_{1}=x^{\prime}\right)-P\left(X_{i}>y \mid X_{1}=x\right) \geq 0
$$

a partir de 2.1.1

$$
f_{X_{1}, X_{i}} T P_{2} \Rightarrow\left(X_{1}, X_{i}\right) S I, i=1,2 \text {. }
$$

$P\left(X_{2}>y \mid X_{1}=x^{\prime}\right)-P\left(X_{2}>y \mid X_{1}=x\right) \geq P\left(X_{3}>y \mid X_{1}=x^{\prime}\right)-P\left(X_{3}>y \mid X_{1}=x\right)$

se, e somente se,

$$
\begin{aligned}
& \int_{y}^{+\infty} f_{X_{1}, X_{2}}\left(x^{\prime}, w\right) d w \int_{-\infty}^{+\infty} f_{X_{1}, X_{2}}(x, z) d z-\int_{y}^{+\infty} f_{X_{1}, X_{2}}(x, w) d w \int_{-\infty}^{+\infty} f_{X_{1}, X_{2}}\left(x^{\prime}, z\right) d z \\
& \geq \int_{y}^{+\infty} f_{X_{1}, X_{3}}\left(x^{\prime}, w\right) d w \int_{-\infty}^{+\infty} f_{X_{1}, X_{3}}(x, z) d z-\int_{y}^{+\infty} f_{X_{1}, X_{3}}(x, w) d w \int_{-\infty}^{+\infty} f_{X_{1}, X_{3}}\left(x^{\prime}, z\right) d z
\end{aligned}
$$

se, e somente se,

$$
\begin{aligned}
& \int_{y}^{+\infty} \int_{-\infty}^{y}\left[f_{X_{1}, X_{2}}\left(x^{\prime}, w\right) f_{X_{1}, X_{2}}(x, z)-f_{X_{1}, X_{2}}(x, w) f_{X_{1}, X_{2}}\left(x^{\prime}, z\right)\right] d z d w \\
\geq & \int_{y}^{+\infty} \int_{-\infty}^{y}\left[f_{X_{1}, X_{3}}\left(x^{\prime}, w\right) f_{X_{1}, X_{3}}(x, z)-f_{X_{1}, X_{3}}(x, w) f_{X_{1}, X_{3}}\left(x^{\prime}, z\right)\right] d z d w
\end{aligned}
$$


Seja $\left(X_{1}, X_{2}, X_{3}\right)$ um vetor trivariado com f.d.p. dada por $f_{X_{1}, X_{2}, X_{3}}$ e $\varphi: R^{2} \rightarrow R$ tal que $U:=\varphi\left(X_{2}, X_{3}\right)$ constitui uma variável aleatória, e a f.d.a. $F_{X_{1}, U}$ é absolutamente contínua. O objetivo é estabelecer condições para poder afirmar:

$$
E_{X_{1}, U}\left(F_{X_{1}, U}\left(X_{1}, U\right)\right) \geq E_{X_{1}, X_{i}}\left(F_{X_{1}, X_{i}}\left(X_{1}, X_{i}\right)\right), i=2,3
$$

em outros termos

$$
\tau_{X_{1}, \varphi\left(X_{2}, X_{3}\right)} \geq \max \left\{\tau_{X_{1}, X_{2}}, \tau_{X_{1}, X_{3}}\right\}
$$

Consideraremos

$$
\varphi\left(x_{2}, x_{3}\right)=x_{2}+x_{3}, \log 0 f_{X_{1}, \varphi\left(X_{2}, X_{3}\right)}(u, w)=\int f_{X_{1}, X_{2}, X_{3}}(u, v, w-v) d v
$$

enquanto que

$$
\begin{aligned}
F_{X_{1}, \varphi\left(X_{2}, X_{3}\right)}\left(x_{1}, x\right) & =\int_{-\infty}^{x_{1}} \int_{-\infty}^{x}\left\{\int f_{X_{1}, X_{2}, X_{3}}(u, v, w-v) d v\right\} d w d u \\
F_{X_{1}, X_{2}}\left(x_{1}, x_{2}\right) & =\int_{-\infty}^{x_{1}} \int_{-\infty}^{x_{2}}\left\{\int f_{X_{1}, X_{2}, X_{3}}(u, v, w) d w\right\} d v d u \\
F_{X_{1}, X_{3}}\left(x_{1}, x_{3}\right) & =\int_{-\infty}^{x_{1}} \int_{-\infty}^{x_{3}}\left\{\int f_{X_{1}, X_{2}, X_{3}}(u, v, w) d v\right\} d w d u
\end{aligned}
$$

\subsubsection{Proposição*}

Seja $\left(X_{1}, X_{2}, X_{3}\right)$ com f.d.p. conjunta $f_{X_{1}, X_{2}, X_{3}} \cdot \varphi: R^{2} \rightarrow R, \varphi(x, y)=x+y$. Supondo que $\forall u, v, w$,

$$
f_{X_{1}, X_{2}, X_{3}}(u, v, w-v) \geq \max \left\{f_{X_{1}, X_{2}, X_{3}}(u, w, v), f_{X_{1}, X_{2}, X_{3}}(u, v, w)\right\} .
$$

Então,

$$
\tau_{X_{1}, X_{2}+X_{3}} \geq \max \left\{\tau_{X_{1}, X_{2}}, \tau_{X_{1}, X_{3}}\right\}
$$


Prova de 4.1.19:

sejam

$$
\begin{gathered}
I:=E_{X_{1}, U}\left(F_{X_{1}, U}\left(X_{1}, U\right)\right), U:=\varphi\left(X_{2}, X_{3}\right) \\
I I:=E_{X_{1}, X_{2}}\left(F_{X_{1}, X_{2}}\left(X_{1}, X_{2}\right)\right) \\
I I I:=E_{X_{1}, X_{3}}\left(F_{X_{1}, X_{3}}\left(X_{1}, X_{3}\right)\right) .
\end{gathered}
$$

Para a prova consideraremos a seguinte notação: $f_{1,2,3}=f_{X_{1}, X_{2}, X_{3}}$, assim como $f_{i, j}=f_{X_{i}, X_{j}}$. Além disso, quando não for especificado, os limites da integração serão $-\infty$ e $+\infty$.

$$
\begin{aligned}
I & =\iint^{x_{1}} \iiint \int^{x_{2}} f_{1,2,3}(u, v, w-v) f_{1,2,3}\left(x_{1}, k, x_{2}-k\right) d w d x_{2} d k d v d u d x_{1} \\
I I & =\iint^{x_{1}} \iiint \int^{x_{2}} f_{1,2,3}(u, w, v) f_{1,2,3}\left(x_{1}, x_{2}, k\right) d w d x_{2} d k d v d u d x_{1} \\
I I I & =\iint^{x_{1}} \iiint \int^{x_{2}} f_{1,2,3}(u, v, w) f_{1,2,3}\left(x_{1}, k, x_{2}\right) d w d x_{2} d k d v d u d x_{1} .
\end{aligned}
$$

Como, por hipótese,

$$
f_{1,2,3}(u, v, w-v) \geq \max \left\{f_{1,2,3}(u, w, v), f_{1,2,3}(u, v, w)\right\},
$$

então,

$$
\begin{aligned}
f_{1,2,3}(u, v, w-v) & \geq \max \left\{f_{1,2,3}(u, w, v), f_{1,2,3}(u, v, w)\right\}, w \leq x_{2}, u \leq x_{1} \\
f_{1,2,3}\left(x_{1}, k, x_{2}-k\right) & \geq \max \left\{f_{1,2,3}\left(x_{1}, x_{2}, k\right), f_{1,2,3}\left(x_{1}, k, x_{2}\right)\right\}
\end{aligned}
$$


A partir daí, temos

$$
\begin{aligned}
& f_{1,2,3}(u, v, w-v) f_{1,2,3}\left(x_{1}, k, x_{2}-k\right) \geq f_{1,2,3}(u, w, v) f_{1,2,3}\left(x_{1}, x_{2}, k\right), w \leq x_{2}, u \leq x_{1} \\
& f_{1,2,3}(u, v, w-v) f_{1,2,3}\left(x_{1}, k, x_{2}-k\right) \geq f_{1,2,3}(u, v, w) f_{1,2,3}\left(x_{1}, k, x_{2}\right), w \leq x_{2}, u \leq x_{1} .
\end{aligned}
$$

Finalmente, $\tau_{X_{1}, X_{2}+X_{3}} \geq \max \left\{\tau_{X_{1}, X_{2}} ; \tau_{X_{1}, X_{3}}\right\}$.

Comentário: Considerando que $X_{1}$ é a variável a predizer e $\left(X_{2}, X_{3}\right)$ a base da predição, a Proposição anterior adquire valor, ou seja a soma dos elementos da base de predição representa uma adequada redução da dimensão dos elementos da base, quando o comportamento de $X_{2}$ e $X_{3}$ - desde a densidade conjunta - for equivalente, isto é: pontualmente $f_{1,2,3}(u, w, v)$ é $f_{1,2,3}(u, v, w)$ são limitados superiormante por $f_{1,2,3}(u, v, w-v)$. 
160

\subsection{Medida $\tau$-Kendall no caso discreto}

Exemplo i: $p(x, y)$

\begin{tabular}{|c|c|c|c|}
\hline \hline $\mathrm{Y} \backslash \mathrm{X}$ & 0 & 1 & $f_{2}(\cdot)$ \\
\hline 0 & $1 / 2$ & 0 & $1 / 2$ \\
\hline 1 & 0 & $1 / 2$ & $1 / 2$ \\
\hline$f_{1}(\cdot)$ & $1 / 2$ & $1 / 2$ & 1 \\
\hline \hline
\end{tabular}

Características:

(1) $P(x, y)=\min \left\{F_{1}(x), F_{2}(y)\right\}, P(X=Y)=1$

(2) As variáveis $X$ e $Y$ são $P A\left(T P_{2}\right)$.

(3) $\tau_{X, Y}=1 ; \quad T_{X, Y}=0,25$

(4) Violação de 3.2.8 [Nelsen (1992)]: $\tau \neq 2 T$.

Exemplo ii: $p(x, y)$

\begin{tabular}{|c|c|c|c|}
\hline \hline $\mathrm{Y} \backslash \mathrm{X}$ & 0 & 1 & $f_{2}(\cdot)$ \\
\hline 0 & $1 / 4$ & 0 & $1 / 4$ \\
\hline 1 & 0 & $3 / 4$ & $3 / 4$ \\
\hline$f_{1}(\cdot)$ & $1 / 4$ & $3 / 4$ & 1 \\
\hline \hline
\end{tabular}


Características:

(1) $P(x, y)=\min \left\{F_{1}(x), F_{2}(y)\right\}, P(X=Y)=1$

(2) As variáveis $X$ e $Y$ são $P A\left(T P_{2}\right)$.

(3) $\tau_{X, Y}=1 ; \quad T_{X, Y}=0,19$.

(4) Violação de 3.2 .8 [Nelsen (1992)] : $\tau \neq 2 T$.

Notemos que em $(i)$ e (ii) toda a probabilidade está concentrada na diagonal usual.

Exemplo iii: $p(x, y)$

\begin{tabular}{|c|c|c|c|}
\hline \hline $\mathrm{Y} \backslash \mathrm{X}$ & 0 & 1 & $f_{2}(\cdot)$ \\
\hline 0 & $1 / 4$ & $1 / 4$ & $2 / 4$ \\
\hline 1 & 0 & $2 / 4$ & $2 / 4$ \\
\hline$f_{1}(\cdot)$ & $1 / 4$ & $3 / 4$ & 1 \\
\hline \hline
\end{tabular}

Características:

(1) $P(x, y)=\min \left\{F_{1}(x), F_{2}(y)\right\}$.

(2) As variáveis $X$ e $Y$ são $P A\left(T P_{2}\right)$.

(3) $\tau_{X, Y}=1 ; \quad T_{X, Y}=0,13$. 
(4) Violação de $3.2 .8[$ Nelsen $(1992)]: \tau \neq 2 T$.

Violação de (ii) de 4.1.1: $P(X=Y) \neq 1$, ao passo que verifica $(i)$ e $(i i i)$.

Exemplo iv: $p(x, y)$

\begin{tabular}{|c|c|c|c|}
\hline \hline $\mathrm{Y} \backslash \mathrm{X}$ & 0 & 1 & $f_{2}(\cdot)$ \\
\hline 0 & $6 / 16$ & $1 / 16$ & $7 / 16$ \\
\hline 1 & $2 / 16$ & $7 / 16$ & $9 / 16$ \\
\hline$f_{1}(\cdot)$ & $8 / 16$ & $8 / 16$ & 1 \\
\hline \hline
\end{tabular}

Características:

(1) As variáveis $X$ e $Y$ são $P A\left(T P_{2}\right)$.

(2) $\tau_{X, Y}=0,97 ; \quad T_{X, Y}=0,16$

(3) Violação de 3.2.8 [Nelsen(1992)].

Exemplo v: $p(x, y)$

\begin{tabular}{|c|c|c|c|}
\hline \hline $\mathrm{Y} \backslash \mathrm{X}$ & 0 & 1 & $f_{2}(\cdot)$ \\
\hline 0 & $1 / 4$ & $1 / 4$ & $1 / 2$ \\
\hline 1 & $1 / 4$ & $1 / 4$ & $1 / 2$ \\
\hline$f_{1}(\cdot)$ & $1 / 2$ & $1 / 2$ & 1 \\
\hline \hline
\end{tabular}


Características:

(1) As variáveis são independentes.

(2) As variáveis $X$ e $Y$ são $P A\left(T P_{2}\right)$.

(3) $\tau_{X, Y}=0,75 ; \quad T_{X, Y}=0$.

(4) Violação de 3.2.8 [Nelsen(1992)].

Exemplo vi: $p(x, y)$

\begin{tabular}{|c|c|c|c|}
\hline \hline $\mathrm{Y} \backslash \mathrm{X}$ & 0 & 1 & $f_{2}(\cdot)$ \\
\hline 0 & $1 / 16$ & $7 / 16$ & $8 / 16$ \\
\hline 1 & $6 / 16$ & $2 / 16$ & $8 / 16$ \\
\hline$f_{1}(\cdot)$ & $7 / 16$ & $9 / 16$ & 1 \\
\hline \hline
\end{tabular}

Características:

(1) As variáveis $X$ e $Y$ são $N A\left(R R_{2}\right)$.

(2) $\tau_{X, Y}=0,34 ; \quad T_{X, Y}=-0,16$.

(3) Violação de 3.2.8 [Nelsen(1992)]: $\tau>0$. 
Exemplo vii: $p(x, y)$

\begin{tabular}{|c|c|c|c|}
\hline \hline $\mathrm{Y} \backslash \mathrm{X}$ & 0 & 1 & $f_{2}(\cdot)$ \\
\hline 0 & 0 & $1 / 2$ & $1 / 2$ \\
\hline 1 & $1 / 4$ & $1 / 4$ & $1 / 2$ \\
\hline$f_{1}(\cdot)$ & $1 / 4$ & $3 / 4$ & 1 \\
\hline \hline
\end{tabular}

Características:

(1) $P(x, y)=\max \left\{0, F_{1}(x)+F_{2}(y)-1\right\}$.

(2) As variáveis $X$ e $Y$ são $N A\left(R R_{2}\right)$.

(3) $\tau_{X, Y}=0,50 ; \quad T_{X, Y}=-0,13$.

(4) Violação de $3.2 .8[\operatorname{Nelsen}(1992)]: \tau>0$.

Violação da condição (i) e (ii) de 4.1.8, ao passo que verifica (iii) da mesma.

Exemplo viii: $p(x, y)$

\begin{tabular}{|c|c|c|c|}
\hline \hline $\mathrm{Y} \backslash \mathrm{X}$ & 0 & 1 & $f_{2}(\cdot)$ \\
\hline 0 & 0 & $3 / 4$ & $3 / 4$ \\
\hline 1 & $1 / 4$ & 0 & $1 / 4$ \\
\hline$f_{1}(\cdot)$ & $1 / 4$ & $3 / 4$ & 1 \\
\hline \hline
\end{tabular}


Características:

(1) $P(x, y)=\max \left\{0, F_{1}(x)+F_{2}(y)-1\right\}, \quad P(X=1-Y)=1$.

(2) As variáveis $X$ e $Y$ são $N A\left(R R_{2}\right)$.

(3) $\tau_{X, Y}=0,25 ; \quad T_{X, Y}=-0,19$.

(4) Verifica (ii) e (iii), de 4.1.8, mas viola (i) da mesma.

Violação de 3.2.8 [Nelsen (1992)].

Exemplo ix: $p(x, y)$

\begin{tabular}{|c|c|c|c|}
\hline \hline $\mathrm{Y} \backslash \mathrm{X}$ & 0 & 1 & $f_{2}(\cdot)$ \\
\hline 0 & 0 & $1 / 2$ & $1 / 2$ \\
\hline 1 & $1 / 2$ & 0 & $1 / 2$ \\
\hline$f_{1}(\cdot)$ & $1 / 2$ & $1 / 2$ & 1 \\
\hline \hline
\end{tabular}

Características:

(1) $P(x, y)=\max \left\{0, F_{1}(x)+F_{2}(y)-1\right\}, \quad P(X=1-Y)=1$

(2) As variáveis $X$ e $Y$ são $N A\left(R R_{2}\right)$.

(3) $\tau_{X, Y}=0 ; \quad T_{X, Y}=-0,25$.

(4) Violação de 4.1 .8 (i) e de 3.2.8. 
"Con este tango que es burlón y compadrito,

se ató dos alas la emoción de mi suburbio.

Con este tango nació el tango y como un grito

salió del sórdido barrial buscando el cielo.

Conjuro extraño de un amor hecho cadencia

que abrió caminos sin más ley que su esperanza

mezcla de rabia, de dolor, de fe, de ausencia,

llorando en la inocencia de un ritmo juguetón..."

Enrique Santos Discépolo y Juan Carlos Marambio Catán, "El Choclo".

\section{Capítulo 5 \\ Associação e Predição}

- Seção 5.1: Construção de um Preditor

- Seção 5.2: Adequacidade

- Seção 5.3: Predição Empírica 


\subsection{Construção de um Preditor}

Nesta Seção, a idéia que vai ser explorada é: se tivermos um vetor aleatório $\left(X_{1}, X_{2}, \cdots, X_{n}\right)$ onde $\left(X_{2}, \cdots, X_{n}\right)$ é a coleção de variáveis na base das quais pretendemos predizer $X_{1}$; variável aleatória que decorre das anteriores, a "informação" contida em $\left(X_{2}, \cdots, X_{n}\right)$ pode ser quantificada pela distribuição da $n$-1 upla de variáveis, $F_{X_{2}, \cdots, X_{n}}$, a mesma contém a "informação" unidimensional mais completa - do ponto de vista probabilístico. Em princípio, para a predição de $X_{1}$ procuramos um preditor unidimensional, sendo que $X_{2}, \cdots, X_{n}$ fornecem tudo o que é preciso para a definição de $X_{1}$. Analisamos $U_{2}=F_{X_{2}, \cdots, X_{n}}\left(X_{2}, \cdots, X_{n}\right)$, f.d.a. aplicada na base $\left(X_{2}, \cdots, X_{n}\right)$, como quantificador da informação da base.

Embora $U_{2}$ esteja alocado entre 0 e 1 , ele reproduz o comportamento de $X_{1}$, salvo escala e alocamento. Os ajustes feitos sobre $U_{2}$ para se tornar um preditor vão depender do banco de dados - histórico - de $X_{1}$ (experiências prévias da $n$ upla completa).

Consideremos as variáveis aleatórias $X_{1}, \cdots, X_{n}$ todas elas designando para cada $\omega \in \mathcal{X}$ os valores, $X_{i}(\omega), i=1, \cdots, n$ respectivamente, os quais podem ser denotados simplesmente por $x_{i}, i=1, \cdots, n$, respectivamente. Naturalmente, podemos considerar a $\sigma$-álgebra induzida por $\left\{X_{1}, \cdots, X_{n}\right\}$, isto é, $\sigma\left(X_{1}, \cdots, X_{n}\right)$, a saber, a menor $\sigma$-álgebra que faz mensuráveis ao vetor $\left(X_{1}, \cdots, X_{n}\right)$.

Adotando $\mathcal{A}:=\sigma\left(X_{1}, \cdots, X_{n}\right)$, temos logo o espaço amostral $(\mathcal{X}, \mathcal{A})$. 


\subsubsection{Lema*}

Seja $\underline{X}=\left(X_{1}, \cdots, X_{n}\right)$ um vetor aleatório no espaço amostral $(\mathcal{X}, \mathcal{A})$, com f.d.a. $F_{\underline{X}}$ contínua. Então, $U(\omega):=F_{\underline{X}}\left(X_{1}(\omega), \cdots, X_{n}(\omega)\right), \omega \in \mathcal{X}$, é uma variável aleatória.

Prova de 5.1.1:

para cada

$$
X_{i}:(\mathcal{X}, \mathcal{A}) \rightarrow(\mathcal{R}, \mathcal{B}(\mathcal{R}))
$$

e $B \in \mathcal{B}(\mathcal{R})$, boreliano da reta, $X_{i}^{-1}(B) \in \mathcal{A}$.

Seja $S \in \mathcal{B}(\mathcal{R})$. Consideremos

$A=\left\{\omega \in \mathcal{X}: F_{X_{1}, \cdots, X_{n}}\left(X_{1}(\omega), \cdots, X_{n}(\omega)\right) \in S\right\}$.

Assim,

$$
A=\left\{\omega \in \mathcal{X}:\left(X_{1}(\omega), \cdots, X_{n}(\omega)\right) \in F_{X_{1}, \cdots, X_{n}}^{-1}(S)\right\}
$$

Suponhamos primeiro que $S$ é aberto.

Mas, $F_{X_{1}, \cdots, X_{n}}: R^{n} \rightarrow R$ é contínua, então sua preimagem $\left(F_{X_{1}, \cdots, X_{n}}^{-1}(S)\right)$ é aberta, para os abertos $S$ em $R$. Logo, $F_{X_{1}, \cdots, X_{n}}^{-1}(S)$ é um boreliano em $R^{n}$. Finalmente $A \in \sigma\left(X_{1}, \cdots, X_{n}\right)=\mathcal{B}$. 
Notemos que se $S=[a, b)$, então

$$
\begin{aligned}
F_{X_{1}, \cdots, X_{n}}^{-1}(S) & =F_{X_{1}, \cdots, X_{n}}^{-1}\left(\bigcap_{n=1}^{\infty}\left(a-\frac{1}{n}, b\right)\right) \\
& =\bigcap_{n=1}^{\infty}\left\{F_{X_{1}, \cdots, X_{n}}^{-1}\left(a-\frac{1}{n}, b\right)\right\}
\end{aligned}
$$

Novamente, $F_{X_{1}, \cdots, X_{n}}^{-1}\left(a-\frac{1}{n}, b\right)$ é aberto em $R^{n}$.

Finalmente, $A \in \sigma\left(X_{1}, \cdots, X_{n}\right)$, quando $S$ é um boreliano.

\subsubsection{Lema*}

Seja $\underline{X}=\left(X_{1}, \cdots, X_{n}\right)$ um vetor aleatório com $f . d . a . F_{X}$ contínua. Suponhamos ainda, que exista uma seqüência ordenada de subíndices $\left\{i_{j}\right\}_{j=1}^{n-1} \subseteq\{1, \cdots, n\}, i_{k}<i_{s}$ sempre que $k<s$, tal que $P\left(\left\{\underline{x}: F_{\underline{X}}(\underline{x})=u \mid X_{i_{1}}=x_{i_{1}}^{0}, \cdots, X_{i_{n-1}}=x_{i_{n-1}}^{0}\right\}\right)$ é bem definida para toda $n$-1 upla $\left(x_{i_{1}}^{0}, \cdots, x_{i_{n-1}}^{0}\right)$ na imagem de $\left(X_{i_{1}}, \cdots, X_{i_{n-1}}\right)$ e para todo $u \in(0,1)$ - i.e. $P\left(X_{i_{1}}=x_{i_{1}}^{0}, \cdots, X_{i_{n-1}}=x_{i_{n-1}}^{0}\right) \neq 0$ ou $f_{X_{i_{1}}, \cdots, X_{i_{n-1}}}\left(x_{i_{1}}^{0}, \cdots, x_{i_{n-1}}^{0}\right)$ $\neq 0$. Então, a variável aleatória $U:=F_{\underline{X}}\left(X_{1}, \cdots, X_{n}\right)$ tem função de distribuição acumulada contínua.

Prova de 5.1.2:

denotamos por $\underline{x}$ cada elemento na imagem de $\underline{X}$. Consideremos $u \in(0,1)$. 
Afirmação: caso bivariado,

$$
\forall u \in(0,1), P\left(\left\{\left(x_{1}, x_{2}\right): F_{X_{1}, X_{2}}\left(x_{1}, x_{2}\right)=u\right\}\right)=0
$$

Notemos que esse resultado é naturalmente extendido.

A quantidade

$$
P\left(\left\{\left(x_{1}, x_{2}\right): F_{X_{1}, X_{2}}\left(x_{1}, x_{2}\right)=u\right\}\right)
$$

pode ser exposta como segue:

$$
\begin{aligned}
& =\int_{-\infty}^{+\infty} P\left(\left(X_{1}, X_{2}\right): F_{X_{1}, X_{2}}\left(X_{1}, X_{2}\right)=u \mid X_{2}=y_{0}\right) d F_{X_{2}}\left(y_{0}\right) \\
& =\int_{-\infty}^{+\infty} \frac{P\left(X_{1}: F_{X_{1}, X_{2}}\left(X_{1}, y_{0}\right)=u\right)}{P\left(X_{2}=y_{0}\right)} d F_{X_{2}}\left(y_{0}\right)
\end{aligned}
$$

Na prévia estamos supondo $P\left(X_{2}=y_{0}\right) \neq 0$ ou equivalentemente, se for o caso $f_{X_{2}}\left(y_{0}\right) \neq 0, \forall y_{0} \in \operatorname{Imag}\left(X_{2}\right)$.

A seguir, analisamos o integrando da anterior,

Como $F_{X_{2}}\left(y_{0}\right)>0$, pois $P\left(X_{2}=y_{0}\right) \neq 0$ podemos analisar os seguintes casos:

- se $u>F_{X_{2}}\left(y_{0}\right)$. Então $u>F_{X_{1}, X_{2}}\left(x, y_{0}\right), \forall x$. Logo

$$
P\left(X_{1}: F_{X_{1}, X_{2}}\left(X_{1}, y_{0}\right)=u\right)=0
$$

- se $u<F_{X_{2}}\left(y_{0}\right)$, definamos

$$
\begin{gathered}
h(x):=F_{X_{1}, X_{2}}\left(x, y_{0}\right), \forall x \in \operatorname{Imag}\left(X_{1}\right), \\
h^{q i}(v):=\inf \{t: h(t) \geq v\}, \quad v \in\left[u, F_{X_{2}}\left(y_{0}\right)\right] .
\end{gathered}
$$


Notemos que $\{t: h(t) \geq v\} \neq \emptyset$, pois como

$$
h(t) \rightarrow_{t \rightarrow+\infty} F_{X_{2}}\left(y_{0}\right) \geq v, \forall v \in\left[u, F_{X_{2}}\left(y_{0}\right)\right]
$$

então $\exists M>0$ tal que $\forall t>M, h(t) \geq v$.

Mais ainda, o conjunto $\{t: h(t) \geq v\}$ é limitado inferiormente.

Como $\lim _{x \rightarrow-\infty} F_{X_{1}, X_{2}}\left(x, y_{0}\right)=0, \exists t_{0}$ tal que $h\left(t_{0}\right)<v$, e $t_{0}$ é limitante inferior do conjunto de interesse. Logo, pelo axioma da completitude de $R,\{t: h(t) \geq v\}$ admite ínfimo.

Com os mesmos argumentos de 3.3.2,

$$
h^{q i}(v) \leq t \Leftrightarrow v \leq h(t), \forall v \in\left[u, F_{X_{2}}\left(y_{0}\right)\right]
$$

conseqüencia da continuidade à direita de $h$.

Logo, vale o equivalente 3.3.7, ou seja $h^{q i}$ é contínua à direita no seu domínio de definição.

Consideremos o intervalo arbitrário $[u, u+\epsilon)$ onde $u+\epsilon \in\left[u, F_{X_{2}}\left(y_{0}\right)\right]$. Sabemos que

$$
\begin{gathered}
u \leq h(x) \text { se, e somente se, } h^{q i}(u) \leq x, \\
u+\epsilon>h(x) \text { se, e somente se, } h^{q i}(u+\epsilon)>x, \\
P(\{x: u \leq h(x)<u+\epsilon\})=P\left(x: h^{q i}(u) \leq x<h^{q i}(u+\epsilon)\right) \\
=F_{X_{1}}\left(h^{q i}(u+\epsilon)\right)-F_{X_{1}}\left(h^{q i}(u)\right) \rightarrow_{\epsilon \rightarrow 0} 0
\end{gathered}
$$


A última propriedade decorre da continuidade à direita da $h^{q i}$ e da continuidade da $F_{X_{1}}$.

- se $u=F_{X_{2}}\left(y_{0}\right)$,

$$
\begin{aligned}
P\left(w: F_{X_{1}, X_{2}}\left(X_{1}(w), y_{0}\right)=u\right) \\
\quad=P\left(w: F_{X_{2}}\left(y_{0}\right)=u, F_{X_{1}, X_{2}}\left(X_{1}(w), y_{0}\right)=u, X_{2}(w) \leq y_{0}\right) \\
\leq P\left(w: X_{1}(w) \in\left[x_{0},+\infty\right), X_{2}(w) \leq y_{0}\right), x_{0} \exists \text { pela monotonicidade de } F_{X_{1}, X_{2}} \\
\quad=P\left(w: X_{1}(w) \in\left[x_{0},+\infty\right), X_{2}(w) \in\left(-\infty, y_{0}\right]\right) \\
\quad=F_{X_{2}}\left(y_{0}\right)-F_{X_{1}, X_{2}}\left(x_{0}, y_{0}\right)=0
\end{aligned}
$$

Finalmente, $P(\{x: h(x)=u\})=0$.

Observação: A hipótese deste Lema foi criada para poder fazer uso do mecanismo de definição da quase-inversa, no caso univariado, pudendo deste modo aproveitar o aprendizado na Seção 3 do capítulo 3.

\subsubsection{Proposição*}

Seja $\underline{X}=\left(X_{1}, \cdots, X_{n}\right)$ vetor aleatório, com $X_{1}$ sendo a variável a predizer, $\left(X_{2}, \cdots, X_{n}\right)$ base da predição, tal que a acumulada $F_{X_{2}, \cdots, X_{n}}$ da $(n-1)$ upla $\left(X_{2}, \cdots, X_{n}\right)$ é contínua e verifica as hipóteses de 5.1 .2 , permitindo definir $U_{2}:=$ $F_{X_{2}, \cdots, X_{n}}\left(X_{2}, \cdots, X_{n}\right)$ como v.a. contínua. Assumamos que $F_{X_{1}}$ é contínua. 
Suponhamos ainda que dados dois vetores independentes $\left(X_{2}, \cdots, X_{n}\right)$ e $\left(X_{2}^{\prime}, \cdots\right.$ , $X_{n}^{\prime}$ ) com a mesma distribuição $F_{X_{2}, \cdots, X_{n}}$, verifica-se

$$
X_{i} \geq X_{i}^{\prime} \text { e } X_{j} \geq X_{j}^{\prime} \Rightarrow X_{s} \geq X_{s}^{\prime} \text { q.t.p., } \forall s \neq i, s \neq j, i \neq j, i, j, s \in\{2, \cdots, n\}
$$

i.e., a concordância entre duas variáveis rotuladas por $i$ e $j$ induz a tendência da variável rotulada por $s$.

$\left\{\left(X_{i}-X_{i}^{\prime}\right)\left(X_{j}-X_{j}^{\prime}\right)<0\right\}=\left\{\left(X_{j}-X_{j}^{\prime}\right)\left(U_{2}-U_{2}^{\prime}\right)<0\right\}$ q.t.p. $\forall i \neq j, i, j \in\{2, \cdots, n\}$,

i.e., a discordância entre as variáveis rotuladas por $i$ e $j$ é captada pela variável $U_{2}$. Então,

$$
\tau_{X_{1}, U_{2}} \geq \tau_{X_{1}, X_{i}}, \quad i=2, \cdots, n, \quad U_{2}:=F_{X_{2}, \cdots, X_{n}}\left(X_{2}, \cdots, X_{n}\right) .
$$

\subsubsection{Lema*}

Seja $\left(X_{1}, \cdots, X_{n}\right)$ un vetor aleatório que verifica as hipóteses da proposição anterior. Se $X_{1}$ e $X_{i}$ são $P A\left(T P_{2}\right), i=2, \cdots, n$, então $U_{2}$ como função das variáveis $X_{2}, \cdots, X_{n}$, maximiza a medida $\tau$ (que aqui constitui uma medida de associação), isto é, $U_{2}$ maximiza a associação existente entre $X_{1}$ e cada uma das variáveis $X_{i}, i=2, \cdots, n$.

Prova de 5.1.3: 
Afirmação 1

Seja $\left(X_{1}, X_{2}, X_{3}\right)$ vetor aleatório, com $X_{1}$ variável aleatória a predizer, $\left(X_{2}, X_{3}\right)$ base da predição, tal que a $F_{X_{2}, X_{3}}$ verifica as hipóteses de 5.1.2. Definamos $U(\omega):=$ $F_{X_{2}, X_{3}}\left(X_{2}(\omega), X_{3}(\omega)\right)$ variável aleatória, então,

$$
\tau_{X_{1}, U} \geq \tau_{X_{1}, X_{i}}, \quad i=2,3
$$

sempre que,

$\left\{\left(X_{2}-X_{2}^{\prime}\right)\left(X_{3}-X_{3}^{\prime}\right)<0,\left(X_{i}-X_{i}^{\prime}\right)\left(U-U^{\prime}\right)<0\right\}=\left\{\left(X_{i}-X_{i}^{\prime}\right)\left(U-U^{\prime}\right)<0\right\}$ q.t.p.

$i=2,3$, onde $\left(X_{2}, X_{3}\right)$ e $\left(X_{2}^{\prime}, X_{3}^{\prime}\right)$ são duas realizações independentes da mesma distribuição $F_{X_{2}, X_{3}}$.

\section{Afirmação 2}

Se a função de distribuição acumulada $F_{X_{1}}$ é contínua, então, as respectivas medidas de concordância $\tau_{X_{1}, X_{2}}, \tau_{X_{1}, X_{3}}$ e $\tau_{X_{1}, U}$ constituem medidas de associação pois $U$ já constitui uma v.a. contínua, pela afirmação prévia - e, se $X_{1}, X_{j}, j=2,3$ são $P A\left(T P_{2}\right)$, então, a variável aleatória $U$ maximiza a associação positiva.

Prova da afirmação 1

Seja $U:=F_{X_{2}, X_{3}}\left(X_{2}, X_{3}\right)$. Se consideramos dois pares de vetores independentes e com a mesma distribuição, $\left(X_{1}, U\right)$ e $\left(X_{1}^{\prime}, U^{\prime}\right)$, temos,

$$
\tau_{X_{1}, U}=2 \eta_{X_{1}, U}-1, \quad \eta_{X_{1}, U}=P\left(\left(X_{1}-X_{1}^{\prime}\right)\left(U-U^{\prime}\right) \geq 0\right) .
$$


Analogamente,

$$
\tau_{X_{1}, X_{i}}=2 \eta_{X_{1}, X_{i}}-1, \quad \eta_{X_{1}, X_{i}}=P\left(\left(X_{1}-X_{1}^{\prime}\right)\left(X_{i}-X_{i}^{\prime}\right) \geq 0\right)
$$

o par $\left(X_{1}, X_{i}\right)$ é independente do par $\left(X_{1}^{\prime}, X_{i}^{\prime}\right)$ e ambos têm a mesma distribuição.

$\eta_{X_{1}, U}$

$$
\begin{aligned}
& =P\left(\left(X_{1}-X_{1}^{\prime}\right)\left(U-U^{\prime}\right) \geq 0\right) \\
& =P\left(\left(X_{1}-X_{1}^{\prime}\right)\left(X_{2}-X_{2}^{\prime}\right)^{2}\left(U-U^{\prime}\right) \geq 0\right) \\
& ={ }_{* 1} \quad P\left(\left(X_{1}-X_{1}^{\prime}\right)\left(X_{2}-X_{2}^{\prime}\right) \geq 0,\left(X_{2}-X_{2}^{\prime}\right)\left(U-U^{\prime}\right) \geq 0\right) \\
& +P\left(\left(X_{1}-X_{1}^{\prime}\right)\left(X_{2}-X_{2}^{\prime}\right)<0,\left(X_{2}-X_{2}^{\prime}\right)\left(U-U^{\prime}\right)<0\right) \\
& \geq P\left(\left(X_{1}-X_{1}^{\prime}\right)\left(X_{2}-X_{2}^{\prime}\right) \geq 0,\left(X_{2}-X_{2}^{\prime}\right)\left(U-U^{\prime}\right) \geq 0\right) \\
& =P\left(\left(X_{1}-X_{1}^{\prime}\right)\left(X_{2}-X_{2}^{\prime}\right) \geq 0,\left(X_{2}-X_{2}^{\prime}\right)\left(X_{3}-X_{3}^{\prime}\right)^{2}\left(U-U^{\prime}\right) \geq 0\right) \\
& =P\left(\left(X_{1}-X_{1}^{\prime}\right)\left(X_{2}-X_{2}^{\prime}\right) \geq 0,\left(X_{2}-X_{2}^{\prime}\right)\left(X_{3}-X_{3}^{\prime}\right) \geq 0,\right. \\
& \left.\left(X_{3}-X_{3}^{\prime}\right)\left(U-U^{\prime}\right) \geq 0\right) \\
& +P\left(\left(X_{1}-X_{1}^{\prime}\right)\left(X_{2}-X_{2}^{\prime}\right) \geq 0,\left(X_{2}-X_{2}^{\prime}\right)\left(X_{3}-X_{3}^{\prime}\right)<0,\right. \\
& \left.\left(X_{3}-X_{3}^{\prime}\right)\left(U-U^{\prime}\right)<0\right) \\
& ={ }_{* 2} \quad P\left(\left(X_{1}-X_{1}^{\prime}\right)\left(X_{2}-X_{2}^{\prime}\right) \geq 0,\left(X_{3}-X_{3}^{\prime}\right)\left(U-U^{\prime}\right) \geq 0\right) \\
& +P\left(\left(X_{1}-X_{1}^{\prime}\right)\left(X_{2}-X_{2}^{\prime}\right) \geq 0,\left(X_{2}-X_{2}^{\prime}\right)\left(X_{3}-X_{3}^{\prime}\right)<0,\right. \\
& \left.\left(X_{3}-X_{3}^{\prime}\right)\left(U-U^{\prime}\right)<0\right) \\
& ={ }_{* 3} \quad P\left(\left(X_{1}-X_{1}^{\prime}\right)\left(X_{2}-X_{2}^{\prime}\right) \geq 0,\left(X_{3}-X_{3}^{\prime}\right)\left(U-U^{\prime}\right) \geq 0\right) \\
& +P\left(\left(X_{1}-X_{1}^{\prime}\right)\left(X_{2}-X_{2}^{\prime}\right) \geq 0,\left(X_{3}-X_{3}^{\prime}\right)\left(U-U^{\prime}\right)<0\right) \\
& =\eta_{X_{1}, X_{2}}
\end{aligned}
$$

onde, em $* 1$ é usada a continuidade das marginais acumuladas: $F_{X_{i}}, i=1,2, F_{U}$. 
$* 2$ :

$$
\begin{gathered}
\left(X_{2}-X_{2}^{\prime}\right)\left(X_{3}-X_{3}^{\prime}\right) \geq 0 \Rightarrow\left\{\begin{array}{c}
X_{2} \geq X_{2}^{\prime}, X_{3} \geq X_{3}^{\prime} \\
\text { ou } \\
X_{2}<X_{2}^{\prime}, X_{3}<X_{3}^{\prime}
\end{array} \Rightarrow\right. \\
\left(X_{3}-X_{3}^{\prime}\right)\left(U-U^{\prime}\right) \geq 0 \text { q.t.p. }
\end{gathered}
$$

a última implicação decorre da monotonicidade da f.d.a. $F_{X_{2}, X_{3}}$.

$* 3$ : decorre da hipótese da afirmação 1 para $i=3$.

Com argumentos análogos prova-se que $\eta_{X_{1}, U} \geq \eta_{X_{1}, X_{3}}$.

Finalmente, decorre das anteriores que

$$
\tau_{X_{1}, U} \geq \max \left\{\tau_{X_{1}, X_{2}}, \tau_{X_{1}, X_{3}}\right\}
$$

Notar que uma hipótese alternativa para a prova da afirmação 1 é a seguinte:

$$
\begin{gathered}
\left\{\left(X_{2}-X_{2}^{\prime}\right)\left(X_{3}-X_{3}^{\prime}\right) \geq 0,\left(X_{i}-X_{i}^{\prime}\right)\left(U-U^{\prime}\right) \geq 0\right\}^{c} \\
=\left\{\left(X_{2}-X_{2}^{\prime}\right)\left(X_{3}-X_{3}^{\prime}\right)<0,\left(X_{i}-X_{i}^{\prime}\right)\left(U-U^{\prime}\right)<0\right\} \text { q.t.p. }
\end{gathered}
$$

Num sentido amplo a hipótese alternativa exige a concordância (discordância) simultánea de $X_{2}, X_{3}$ e $U$.

A prova para o caso geral, decorre das mesmas idéias: 
$\eta_{X_{1}, U_{2}}$

$$
\begin{aligned}
& =P\left(\left(X_{1}-X_{1}^{\prime}\right)\left(U_{2}-U_{2}^{\prime}\right) \geq 0\right) \\
& =P\left(\left(X_{1}-X_{1}^{\prime}\right)\left(X_{i}-X_{i}^{\prime}\right)^{2}\left(U_{2}-U_{2}^{\prime}\right) \geq 0\right), n \geq i \geq 2 \\
& =P\left(\left(X_{1}-X_{1}^{\prime}\right)\left(X_{i}-X_{i}^{\prime}\right) \geq 0,\left(X_{i}-X_{i}^{\prime}\right)\left(U_{2}-U_{2}^{\prime}\right) \geq 0\right) \\
& +P\left(\left(X_{1}-X_{1}^{\prime}\right)\left(X_{i}-X_{i}^{\prime}\right)<0,\left(X_{i}-X_{i}^{\prime}\right)\left(U_{2}-U_{2}^{\prime}\right)<0\right) \\
& \geq P\left(\left(X_{1}-X_{1}^{\prime}\right)\left(X_{i}-X_{i}^{\prime}\right) \geq 0,\left(X_{i}-X_{i}^{\prime}\right)\left(U_{2}-U_{2}^{\prime}\right) \geq 0\right) \\
& =P\left(\left(X_{1}-X_{1}^{\prime}\right)\left(X_{i}-X_{i}^{\prime}\right) \geq 0,\right. \\
& \left.\left(X_{i}-X_{i}^{\prime}\right)\left(X_{j}-X_{j}^{\prime}\right)^{2}\left(U_{2}-U_{2}^{\prime}\right) \geq 0\right), \quad j \neq i, n \geq j \geq 2 \\
& =P\left(\left(X_{1}-X_{1}^{\prime}\right)\left(X_{i}-X_{i}^{\prime}\right) \geq 0,\left(X_{i}-X_{i}^{\prime}\right)\left(X_{j}-X_{j}^{\prime}\right) \geq 0,\right. \\
& \left.\left(X_{j}-X_{j}^{\prime}\right)\left(U_{2}-U_{2}^{\prime}\right) \geq 0\right) \\
& +P\left(\left(X_{1}-X_{1}^{\prime}\right)\left(X_{i}-X_{i}^{\prime}\right) \geq 0,\left(X_{i}-X_{i}^{\prime}\right)\left(X_{j}-X_{j}^{\prime}\right)<0,\right. \\
& \left.\left(X_{j}-X_{j}^{\prime}\right)\left(U_{2}-U_{2}^{\prime}\right)<0\right) \\
& ={ }_{* 4} \quad P\left(\left(X_{1}-X_{1}^{\prime}\right)\left(X_{i}-X_{i}^{\prime}\right) \geq 0,\left(X_{j}-X_{j}^{\prime}\right)\left(U_{2}-U_{2}^{\prime}\right) \geq 0\right) \\
& +P\left(\left(X_{1}-X_{1}^{\prime}\right)\left(X_{i}-X_{i}^{\prime}\right) \geq 0,\left(X_{j}-X_{j}^{\prime}\right)\left(U_{2}-U_{2}^{\prime}\right)<0\right) \\
& =\eta_{X_{1}, X_{i}}, \quad n \geq i \geq 2
\end{aligned}
$$

$* 4:\left(X_{i}-X_{i}^{\prime}\right)\left(X_{j}-X_{j}^{\prime}\right) \geq 0 \Rightarrow\left(X_{j}-X_{j}^{\prime}\right)\left(U_{2}-U_{2}^{\prime}\right) \geq 0$ q.t.p., decorre da primeira hipótese da proposição e da monotonicidade da f.d.a. $F_{X_{2}, \cdots, X_{n}}$. Finalmente, pela segunda hipóteses da proposição decorre a igualdade. 
5.1.5 Natureza topológica do preditor: A seguir, apresentamos uma análise feita sobre distribuições em $R^{2}$.

Seja $F_{X_{1}, X_{2}}$ f.d.a. contínua, de $R^{2}$ em $R$. Considere $u \in(0,1)$.

Sejam

$$
A_{u}:=\left\{\underline{x} \in R^{2}: F_{X_{1}, X_{2}}(\underline{x}) \leq u\right\}, B_{u}:=\left\{\underline{x} \in R^{2}: F_{X_{1}, X_{2}}(\underline{x})>u\right\} .
$$

$\pi_{i}: R^{2} \rightarrow R$, a projeção usual na $i$-ésima coordenada. $i=1,2$.

Notemos que $B_{u} \neq \emptyset$, pois $\lim _{x_{i} \rightarrow+\infty i=1,2} F_{X_{1}, X_{2}}\left(x_{1}, x_{2}\right)=1$. Então, dado $\epsilon>$ $0 \exists M$ tal que se $x_{i}>M, i=1,2$ resulta $1-F_{X_{1}, X_{2}}\left(x_{1}, x_{2}\right)<\epsilon$.

Pela arbitrariedade de $\epsilon$, podemos considerar $\epsilon=1-u$. Logo, $B_{u} \neq \emptyset$.

Com argumentos semelhantes, é possível concluir que $A_{u} \neq \emptyset$.

Conseqüentemente é possível fazer

$$
B_{u}^{i}=\pi_{i}\left(B_{u}\right), A_{u}^{i}=\pi_{i}\left(A_{u}\right), i=1,2 .
$$

Mais especificamente,

$$
A_{u}^{1}=\left\{x: \exists y_{x}, F_{X_{1}, X_{2}}\left(x, y_{x}\right) \leq u\right\}, B_{u}^{1}=\left\{x: \exists y_{x}, F_{X_{1}, X_{2}}\left(x, y_{x}\right)>u\right\}
$$

5.1.6 Proposição* : Se $F_{X_{1}, X_{2}}$ é f.d.a. de $R^{2}$ em $R, u \in(0,1), B_{u}^{i}, A_{u}^{i} i=1,2$ definidos com anterioridade. $B_{u}^{i}$ é limitado inferiormente e possui ínfimo $m_{i}^{u}-m_{i}^{u} \in$ $f r\left(B_{u}^{i}\right)$ ( fronteira de $B_{u}^{i}$ ) - $i=1,2$. Enquanto que $A_{u}^{i}, i=1,2$ não é limitado superiormente. 
Prova de 5.1.6:

Notemos que $A_{u}^{1}$ não é limitado superiormente, pois dado $\epsilon>0, \forall x \exists M_{x, \epsilon}$ tal que, se $y<M_{x, \epsilon} \Rightarrow F_{X_{1}, X_{2}}(x, y)<\epsilon$ (pois $\lim _{y \rightarrow-\infty} F_{X_{1}, X_{2}}(x, y)=0$ ), então, $(x, y) \in A_{u}$. Logo, $\{(x, 0)\} \subseteq A_{u}^{1}, \forall x \in R$.

Por outro lado, $B_{u}^{1}$ é limitado inferiormente.

Afirmação $1 \quad L:=\left\{x \in R: u \geq F_{X_{1}}(x)\right\}$ constitui um conjunto de limitantes inferiores de $B_{u}^{1} . L \subseteq A_{u}^{1}, L \cap B_{u}^{1}=\emptyset$.

Prova da Afirmação 1

$L \neq \emptyset$ : pois, $\lim _{x \rightarrow-\infty} F_{X_{1}}(x)=0$, i.e., $\forall \epsilon>0 \exists M_{\epsilon}$ tal que se $x<M_{\epsilon} \Rightarrow F_{X_{1}}(x)<\epsilon$. Logo, se $\epsilon \leq u, x \leq M_{\epsilon}, x \in L$.

Suponhamos agora que existem $x_{0} \in B_{u}^{1}$ e $x_{0}^{\prime} \in L$ tal que $x_{0}<x_{0}^{\prime}$ :

(i) como $x_{0} \in B_{u}^{1} \Rightarrow \exists y_{0}$ tal que $F_{X_{1}, X_{2}}\left(x_{0}, y_{0}\right)>u$, então $F_{X_{1}, X_{2}}\left(x_{0}^{\prime}, y_{0}\right)>u$;

(ii) como $x_{0}^{\prime} \in L \Rightarrow u \geq F_{X_{1}}\left(x_{0}^{\prime}\right) \geq F_{X_{1}, X_{2}}\left(x_{0}^{\prime}, y\right) \forall y \in R$, particularmente, $u \geq$ $F_{X_{1}, X_{2}}\left(x_{0}^{\prime}, y_{0}\right)$.

Logo, por (i) e (ii) conclui-se

$$
u \geq F_{X_{1}, X_{2}}\left(x_{0}^{\prime}, y_{0}\right)>u \text { : absurdo, }
$$


ou seja, que $L$ constitui um conjunto de limitantes inferiores de $B_{u}^{1}$.

Além disso, $L \subseteq A_{u}^{1}, L \cap B_{u}^{1}=\emptyset$.

Primeiramente, se $x \in L, u \geq F_{X_{1}}(x)$ e

$$
u \geq F_{X_{1}}(x) \geq F_{X_{1}, X_{2}}(x, y), \quad \forall y \in R
$$

Ou seja, $L \subseteq A_{u}^{1}$.

Para a última parte da Afrrmação 1, vamos supor que existe $x \in L \cap B_{u}^{1}$,

(i) como $x \in L \Rightarrow F_{X_{1}}(x) \leq u \Rightarrow \forall y, \quad F_{X_{1}, X_{2}}(x, y) \leq u$;

(ii) como $x \in B_{u}^{1} \Rightarrow \exists y_{x}: F_{X_{1}, X_{2}}\left(x, y_{x}\right)>u$.

Logo, (i) e (ii) levam ao absurdo. Finalizando com a prova da Afirmação 1

Notemos que, pelo axioma da completitude de $R$, os conjuntos $B_{u}^{i}, i=1,2$ possuem ínfimo em $R$.

Consideremos $B_{u}^{1}$. Para este conjunto não vazio, existe $m_{1}^{u}$ verificando as seguintes propriedades:

(i) $\forall x \in B_{u}^{1}, m_{1}^{u} \leq x$.

(ii) se $s \in R$ satisfaz, $s \leq x, \forall x \in B_{u}^{1}$. Então $s \leq m_{1}^{u}$. 
Afirmação $2 m_{1}^{u} \in \operatorname{fr}\left\{B_{u}^{1}\right\}$ (fronteira de $B_{u}^{1}$ ).

Prova da Afirmação 2

Como $m_{1}^{u} \in \overline{B_{u}^{1}}$, pois, se supomos que $m_{1}^{u}$ não pertence ao fecho de $B_{u}^{1} \Rightarrow m_{1}^{u} \in$ $R-\overline{B_{u}^{1}}$, i.e.,$\exists \epsilon>0$ tal que $\left(m_{1}^{u}-\epsilon, m_{1}^{u}+\epsilon\right) \subseteq R-\overline{B_{u}^{1}}$. Logo. $\exists v_{\epsilon} \in\left(m_{1}^{u}, m_{1}^{u}+\epsilon\right)$ tal que $v_{\epsilon} \leq x, \forall x \in B_{u}^{1}$, e $v_{\epsilon} \geq m_{1}^{u}:$ absurdo. Por tanto, $m_{1}^{u} \in \overline{B_{u}^{1}}$.

Agora, $\overline{B_{u}^{1}}=\operatorname{int}\left\{B_{u}^{1}\right\} \cup \operatorname{fr}\left\{B_{u}^{1}\right\}$.

Suponhamos que $m_{1}^{u} \in \operatorname{int}\left\{B_{u}^{1}\right\}$. Então, $\exists \epsilon>0$ tal que $\left(m_{1}^{u}-\epsilon, m_{1}^{u}+\epsilon\right) \subseteq B_{u}^{1}$, $\Rightarrow$ $\exists x \in\left(m_{1}^{u}-\epsilon, m_{1}^{u}\right) \subseteq B_{u}^{1}$ : absurdo, pois, para todo $x \in B_{u}^{1}, x \geq m_{1}^{u}$. Logo, $m_{1}^{u} \notin$ $\operatorname{int}\left\{B_{u}^{1}\right\}$.

Finalmente

$$
m_{1}^{u} \in \operatorname{fr}\left\{B_{u}^{1}\right\}
$$

Comentário: As espectativas perante o estudo topológico dos conjuntos $A_{u}$ e $B_{u}$ são ambiciosas. Baseados nos resultados obtidos no caso univariado - que foram desenvolvidos na Seção 3 do Capítulo 3 - podemos afirmar que a complexidade atingida por essas estruturas em $R^{n}, n>1$, não é trivial e orienta nossos passos ao trabalho de famílias de distribuições específicas, que permitam estabelecer condições para que partindo de $B_{u}$ possa ser repetido o esquema utilizado no Capítulo 3, com o objetivo de que o par de variáveis $X_{1}, X_{2} \operatorname{com} f$.d.a. $F_{X_{1}, X_{2}}$ possa ser totalmente ca- 
racterizado pelos valores atingidos pela distribuição acumulada $F_{X_{1}, X_{2}}$ no domínio de definição da mesma. O objeto de tal pretenção tem sua justificativa: a distribuição de um vetor aleatório é o melhor resumo para fazer inferência respeito dos dados. Por outro lado se procuramos uma redução da dimensão dos dados, precisamos de alguma proposta que possa condensar um vetor de dados em alguma estrutura de dimensão menor, mais não por isso menos informativa, nesse ponto a distibuição acumulada pode apresentar uma alternativa viável. 


\subsection{Adequacidade}

Consideremos o espaço amostral $(\mathcal{X}, \mathcal{A})$, munido de uma família de distribuições de probabilidade $\mathcal{P}$. Seja $\mathcal{B}$ uma sub $\sigma$-algébra da $\sigma$-álgebra $\mathcal{A}$. Dizemos que $\mathcal{B}$ é suficiente para $\mathcal{A}$ em relação à familia $\mathcal{P}$, se para cada $A \in \mathcal{A}$ existe uma função $\mathcal{B}$-mensurável, digamos $g_{A}$, sobre $\mathcal{X}$ tal que

$$
P(A \cap B)=\int_{B} g_{A} d P, \quad \forall B \in \mathcal{B} \text { e } \forall P \in \mathcal{P}
$$

Se consideramos o caso particular, onde $\mathcal{P}=\left\{P_{0}\right\}$, dispomos do seguinte resultado

\subsubsection{Teorema [Ash (1972) pp.253]}

Seja $\left(\mathcal{X}, \mathcal{A},\left\{P_{0}\right\}\right)$ espaço de probabilidade, $\mathcal{B} \subseteq \mathcal{A}$, sub $\sigma$-álgebra. $\forall A \in \mathcal{A}, A$ fixo, existe uma função $P_{0}(A \mid \mathcal{B}):(\mathcal{X}, \mathcal{B}) \rightarrow(\mathcal{R}, \mathcal{B}(\mathcal{R})),(\mathcal{B}-\mathcal{B}(\mathcal{R}))$ mensurável, chamada probabilidade $\left(P_{0}\right)$ condicional de $A$ dada $\mathcal{B}$, tal que

$$
P_{0}(A \cap B)=\int_{B} P_{0}(A \mid \mathcal{B}) d P_{0}, \quad \forall B \in \mathcal{B}
$$

Assim, freqüentemente definimos

$$
E_{P_{0}}\left(I_{A} \mid \mathcal{B}\right):=P_{0}(A \mid \mathcal{B}), \forall A \in \mathcal{A}
$$

Apresentamos, a seguir, uma prova resumida do teorema anterior.

Definamos $\lambda(B):=P_{0}(B \cap A)$, para cada $B \in \mathcal{B}$, com $A \in \mathcal{A}$ fixado previamente. Notemos que $\lambda<<P_{0}$. 
O Teorema de Radom-Nykodim garante que, consirerando $(\mathcal{X}, \mathcal{B})$, existe uma função $(\mathcal{B}-\mathcal{B}(\mathcal{R}))$ mensurável, $g:(\mathcal{X}, \mathcal{B}) \rightarrow(\mathcal{R}, \mathcal{B}(\mathcal{R}))$, tal que

$$
\lambda(B *)=\int_{B *} g d P_{0}, \forall B * \in \mathcal{B} .
$$

Notemos que $g$, chamada derivada de Radom-Nykodim de $\lambda$ com relação a $P_{0}$, comumente denotada por $\frac{d \lambda}{d P_{0}}$, depende do $A$ fixo no começo da prova. Definamos $P_{0}(A \mid \mathcal{B}):=g$. Para concluir a prova, basta notar que $g$ é $\left.(\mathcal{B})-\mathcal{B}(\mathcal{R})\right)$ mensurável, pelo próprio Teorema de Radom-Nykodim.

Assim, podemos concluir que quando estudamos a situação $\left(\mathcal{X}, \mathcal{A},\left\{P_{0}\right\}\right)$ e consideramos uma sub $\sigma$-álgebra de $\mathcal{A}$, digamos $\mathcal{B}$, a suficiencia é sempre possível, pois sempre podemos fabricar para cada $A \in \mathcal{A}$ uma medida $\lambda_{A}(B):=P_{0}(A \cap B)$ sobre $\mathcal{B}$ que será dominada absolutamente por $P_{0}$. Portanto, existirá a $\frac{d \lambda_{A}}{d P_{0}}$ função com domínio em $(\mathcal{X}, \mathcal{B})$ e imagem de chegada em $(\mathcal{R}, \mathcal{B}(\mathcal{R})), \quad(\mathcal{B}-\mathcal{B}(\mathcal{R}))$ mensurável, que verifica

$$
\begin{aligned}
P_{0}(A \cap B) & =\lambda_{A}(B) \\
& =\int_{B} \frac{d \lambda_{A}}{d P_{0}} d P_{0}, \forall B \in \mathcal{B} .
\end{aligned}
$$

Apresentamos em seguida outro conceito da teoria de suficiencidade [Basu e Cheng (1981)].

Daqui para frente nos permitimos a notação reduzida $\mathcal{A}$ mensurável quando fizermos menção a $(\mathcal{A}-\mathcal{B}(\mathcal{R}))$ mensurável. 
5.2.2 Adequacidade: Seja $\left(\mathcal{X}, \mathcal{A},\left\{P_{0}\right\}\right)$ um espaço de probabilidade, $\mathcal{B}$ e $\mathcal{C}$ duas sub $\sigma$-álgebras da $\sigma$-álgebra $\mathcal{A}$. Sem perda da generalidade podemos tomar $\mathcal{A}=\mathcal{B} \vee \mathcal{C}$; onde $\mathcal{A}$ é a menor $\sigma$-álgebra contendo a união de $\mathcal{B}$ e $\mathcal{C}$. Consideremos $\mathcal{B}_{0}$ sub $\sigma$-álgebra da $\mathcal{B}$. Dizemos que $\mathcal{B}_{0}$ é adequada para predizer $\mathcal{C}$ na base de $\mathcal{B}$, se para cada $C \in \mathcal{C}$ existe uma versão $\mathcal{B}_{0}$-mensurável da $E_{P_{0}}\left(I_{C} \mid \mathcal{B}\right)$.

Notação: $\mathcal{B}_{0} \operatorname{adq}\left(\mathcal{B} ; \mathcal{C}, P_{0}\right)$

Notemos que, pelo Teorema 1 (Skibinsky (1966)), temos, $\mathcal{B}_{0} \operatorname{adq}\left(\mathcal{B} ; \mathcal{C}, P_{0}\right)$ se, e somente se, dado $B \in \mathcal{B}$, existe uma versão da $E_{P_{0}}\left(I_{B} \mid \mathcal{B}_{0} \vee \mathcal{C}\right), \mathcal{B}_{0}$-mensurável.

Se $B \in \mathcal{B}$, então, $I_{B}$ é uma variável aleatória sobre $\left(\mathcal{X}, \mathcal{A},\left\{P_{0}\right\}\right)$. Dada a $\sigma$-álgebra $\mathcal{B}_{0} \vee \mathcal{C}$ contida em $\mathcal{A}$, existe uma função,

$$
E_{P_{0}}\left(I_{B} \mid \mathcal{B}_{0} \vee \mathcal{C}\right):\left(\mathcal{X}, \mathcal{B}_{0} \vee \mathcal{C}\right) \rightarrow(\mathcal{R}, \mathcal{B}(\mathcal{R}))
$$

tal que $\forall D \in \mathcal{B}_{0} \vee \mathcal{C}$

$$
\int_{D} I_{B} d P_{0}=\int_{D} E_{P_{0}}\left(I_{B} \mid \mathcal{B}_{0} \vee \mathcal{C}\right) d P_{0}
$$

Pelo Teorema 6.4.3 de Ash pp.252, segue-se que, $E_{P_{0}}\left(I_{B} \mid \mathcal{B}_{0} \vee \mathcal{C}\right)$ é $P_{0}$-integrável e $\mathcal{B}_{0} \vee \mathcal{C}$-mensurável. 
Por outro lado, para um espaço probabilístico $\left(\mathcal{X}, \mathcal{A},\left\{P_{0}\right\}\right)$ dotado de três sub $\sigma$-álgebras de $\mathcal{A} ; \mathcal{F}, \mathcal{G}, \mathcal{H}$. Verifica-se

$$
\mathcal{F} \| \mathcal{G} \mid \mathcal{H} \text { q.t.p. }\left[P_{0}\right]
$$

$(\mathcal{F}$ independente de $\mathcal{G}$ dada $\mathcal{H})$

se, e somente se, para cada $G \in \mathcal{G}$, existe uma versão $\mathcal{H}$-mensurável de

$$
E_{P_{0}}\left(I_{G} \mid \mathcal{F} \vee \mathcal{H}\right)
$$

[Loéve(1963)].

Aplicando este resultado para $\mathcal{F}=\mathcal{C}, \mathcal{G}=\mathcal{B}, \mathcal{H}=\mathcal{B}_{0}$, temos o seguinte Lema,

\subsubsection{Lema}

$$
\mathcal{C} \| \mathcal{B} \mid \mathcal{B}_{0} \text { q.t.p. }\left[P_{0}\right]
$$

se, e somente se, para cada $B \in \mathcal{B}$, existe uma versão $\mathcal{B}_{0}$-mensurável de

$$
E_{P_{0}}\left(I_{B} \mid \mathcal{B}_{0} \vee \mathcal{C}\right)
$$

Vale dizer que as seguintes condições são equivalentes 


\subsubsection{Teorema}

Seja $\left(\mathcal{X}, \mathcal{A},\left\{P_{0}\right\}\right)$ um espaço probabilístico, $\mathcal{B}, \mathcal{C}$ e $\mathcal{B}_{0}$, sub $\sigma$-álgebras de $\mathcal{A}$, tal que $\mathcal{B}_{0} \subseteq \mathcal{B}$. Então, as seguintes condições são equivalentes,

(i) $\mathcal{B}_{0} \operatorname{adq}\left(\mathcal{B} ; \mathcal{C}, P_{0}\right)$;

(ii) para cada $B \in \mathcal{B}$, existe uma versão $\mathcal{B}_{0}$-mensurável da $E_{P_{0}}\left(I_{B} \mid \mathcal{B}_{0} \vee \mathcal{C}\right)$;

(iii) $\mathcal{B} \| \mathcal{C} \mid \mathcal{B}_{0}$ q.t.p. $\left[P_{0}\right]$.

5.2.5 Adequacidade e Predição: Consideremos as variáveis aleatórias e observáveis $X_{2}, X_{3}$ e a variável aleatória não observável $X_{1}$, todas elas designando para cada $\omega \in \mathcal{X}$ os valores, $X_{2}(\omega), X_{3}(\omega)$ e $X_{1}(\omega)$ respectivamente. Naturalmente, podemos considerar as $\sigma$-álgebras induzidas por $\left(X_{2}, X_{3}\right)$ e $X_{1}$, isto é, $\sigma\left(X_{2}, X_{3}\right)$ e $\sigma\left(X_{1}\right)$, a saber, as menores $\sigma$-álgebras que tornam mensuráveis o par $\left(X_{2}, X_{3}\right)$ e a função $X_{1}$, respectivamente.

Adotamos, $\mathcal{B}:=\sigma\left(X_{2}, X_{3}\right), \mathcal{C}:=\sigma\left(X_{1}\right)$. Podemos, pois, considerar $\mathcal{A}:=\mathcal{B} \vee \mathcal{C}$. Suponhamos que a função de distribuição acumulada do par $\left(X_{2}, X_{3}\right), F_{X_{2}, X_{3}}$ é contínua. Consideremos a variável aleatória $U:=F_{X_{2}, X_{3}}\left(X_{2}, X_{3}\right)$. Poderia esta ser uma "boa" preditora da variável não observável $X_{1}$ ?

Adotamos $\mathcal{T}:=\sigma(U)$. É possível notar que $\mathcal{T}$ é sub $\sigma$-álgebra de $\mathcal{B}$.

Seja $A \in \mathcal{T}$. Então, $A=\left\{\omega \in \mathcal{X}: F_{X_{2}, X_{3}}\left(X_{2}(\omega), X_{3}(\omega)\right) \in S\right\}$, onde $S$ é algum 
boreliano da reta.

Assim,

$$
A=\left\{\omega \in \mathcal{X}:\left(X_{2}(\omega), X_{3}(\omega)\right) \in F_{X_{2}, X_{3}}^{-1}(S)\right\}
$$

Mas $F_{X_{2}, X_{3}}: R^{2} \rightarrow R$ é contínua; então, sua preimagem $\left(F_{X_{2}, X_{3}}^{-1}(S)\right)$ é aberta, para os abertos $S$ em $R$. Logo, $F_{X_{2}, X_{3}}^{-1}(S)$ é um boreliano em $R^{2}$. Finalmente, $A \in$ $\sigma\left(X_{2}, X_{3}\right)=\mathcal{B}$.

Logo, $\mathcal{T} \subseteq \mathcal{B}$ e poderia ser uma candidata a $\sigma$-álgebra $\operatorname{adq}\left(\mathcal{B} ; \mathcal{C}, P_{0}\right)$, isto é, $F_{X_{2}, X_{3}}\left(X_{2}, X_{3}\right)$ poderia ser adequada para predizer $X_{1}$ na base observacional dada por $X_{2}, X_{3}$; onde $P_{0}$ é a probabilidade conjunta, a saber $P_{0}\left(X_{1} \in C,\left(X_{2}, X_{3}\right) \in B\right)$ para $C \in \mathcal{C}$ e $B \in \mathcal{B}$.

Em geral, se considerarmos $X_{1}, X_{2}, \cdots, X_{n}$ onde $X_{1}$ é a variável aleatória não observável e $X_{2}, \cdots, X_{n}$ como base para nossa predição de $X_{1}$. $\mathcal{B}=\sigma\left(X_{2}, \cdots, X_{n}\right)$ definimos

$$
\begin{aligned}
& \mathcal{T}_{1}=\sigma\left(F_{X_{2}, \cdots, X_{n}}\left(X_{2}, \cdots, X_{n}\right)\right) \\
& \mathcal{T}_{i}=\sigma\left(X_{2}, \cdots, X_{i}, F_{X_{i+1}, \cdots, X_{n}}\left(X_{i+1}, \cdots, X_{n}\right)\right), i=2, \cdots, n-2
\end{aligned}
$$

Se as $F_{X_{i}, \cdots, X_{n}}, i=2, \cdots, n-2$ são contínuas, então, $\left\{\mathcal{T}_{i}\right\}_{i=1}^{n-2} \subseteq \mathcal{B}$.

Essa seqüência de sub $\sigma$-álgebras de $\mathcal{B}$ apresenta uma seqüência de candidatas adequadas para predizer $X_{1}$ na base observacional de $X_{2}, \cdots, X_{n}$. 


\subsubsection{Teorema*}

Seja $\left(\mathcal{X}, \mathcal{A},\left\{P_{0}\right\}\right)$ um espaço probabilístico, $\left(X_{1}, X_{2}, \cdots, X_{n}\right)$ vetor aleatório com f.d.a. conjunta $F_{X_{1}, \cdots, X_{n}}$ e marginais acumuladas contínuas, $F_{X_{i}, \cdots, X_{n}}, i=2, \cdots, n$. Consideremos as seguintes sub- $\sigma$ álgebras induzidas,

$$
\begin{gathered}
\mathcal{C}=\sigma\left(X_{1}\right), \quad \mathcal{B}=\sigma\left(X_{2}, \cdots, X_{n}\right) \\
\mathcal{T}_{1}=\sigma\left(F_{X_{2}, \cdots, X_{n}}\left(X_{2}, \cdots, X_{n}\right)\right) \\
\mathcal{T}_{i}=\sigma\left(X_{2}, \cdots, X_{i}, F_{X_{i+1}, \cdots, X_{n}}\left(X_{i+1}, \cdots, X_{n}\right)\right), i=2, \cdots, n-2
\end{gathered}
$$

Então, se existe $i_{0} \in\{1, \cdots, n-2\}$ tal que $\mathcal{C} \subseteq \mathcal{T}_{i_{0}}$,

$$
\mathcal{T}_{i_{0}} \text { é } \operatorname{adq}\left(\mathcal{B} ; \mathcal{C}, P_{0}\right) \text {. }
$$

Prova : conseqüência da parte (ii) de 5.2.4.

Notemos que a suposição $\mathcal{C} \subseteq \mathcal{T}_{i_{0}}$ responde à seguinte idéia: $X_{1}$ decorre de $X_{2}, \cdots, X_{n}$, portanto, a "informação" contida em $\mathcal{C}$ é contemplada em alguma sub $\sigma$-álgebra de $\mathcal{B}$.

Centramos nosso estudo em famílias de probabilidades de um único elemento $\left\{P_{0}\right\}$ pois estamos assumindo que somente existe uma probabilidade $P_{0}$ vinculando as variáveis $X_{i}, i=1,2, \cdots, n$. 


\subsection{7 $\tau$ de Kendall, adequacidade e preditor}

Sejam $X_{1}, \cdots, X_{n}$ variáveis aleatórias, cujos valores são obtidos da amostra, $Y$ uma variável resposta. tirada a partir das anteriores. A seguir, apresentamos um exemplo no qual fabricamos uma estatística $T:=X_{1} \ldots X_{n}$ adequada para predizer $Y$ com base na amostra $\underline{X}=\left(X_{1}, \cdots, X_{n}\right)$. Consideremos uma família de distribuições de probabilidade $P=\left\{p_{\theta}, \theta \in \Theta\right\}$. É possível verificar, no exemplo, que $X_{i}, Y, i=$ $1, \cdots, n$ são $P A\left(T P_{2}\right)$ assim como $T, Y$ são $P A\left(T P_{2}\right)$. Assim, passamos a calcular a medida $\tau$ de Kendall, verificando que $\tau_{T, Y} \geq \tau_{X_{i}, Y}, i=1, \cdots, n$.

$$
\begin{aligned}
& \text { Em seguida, } F_{X_{1}, \cdots, X_{n}}\left(X_{1}, \cdots, X_{n}\right)=T^{\theta} \mathrm{e} \\
& \qquad \tau_{T^{\theta}, Y}=\tau_{T, Y} \geq \tau_{X_{i}, Y}, \quad i=1, \cdots, n . \\
& p_{\theta}\left(x_{1}, \cdots, x_{n}, y\right):=\frac{\theta^{n}\left(x_{1} \ldots x_{n}\right)^{\theta-1}}{\pi\left(1+y^{2}\right)}\left\{1+\frac{\theta}{\pi}\left[2\left(x_{1} \ldots x_{n}\right)^{\theta}-1\right] \operatorname{arctg}(y)\right\} \\
& 0<\theta<1,-\infty<y<+\infty, 0 \leq x_{i} \leq 1, i=1, \cdots, n .
\end{aligned}
$$

Se $\underline{X}=\left(X_{1}, \cdots, X_{n}\right)$ e $T=X_{1} \ldots X_{n}$

$$
g_{\theta}(t, y)=\frac{\theta^{n} t^{\theta-1}}{1+y^{2}}\left[1+\frac{\theta}{\pi}\left(2 t^{\theta}-1\right) \operatorname{arctg}(y)\right], \quad h=\frac{1}{\pi}
$$

então, $T$ é $\operatorname{adq}(\underline{X} ; Y, P)$ [Basu e Cheng (1981)]

Em seguida, veremos que $\left(X_{i}, Y\right)$ possui densidade de probabilidade $T P_{2}$, então, as variáveis aleatórias são $P A\left(T P_{2}\right), i=1, \cdots, n$. 
Provemos a afirmação para $i=1$, sem perda da generalidade,

$$
\begin{aligned}
p_{\theta}(\underline{x}, y)= & \frac{\theta^{n}}{\pi\left(1+y^{2}\right)}\left[1-\frac{\theta}{\pi} \operatorname{arctg}(y)\right] x_{1}^{\theta-1}\left(x_{2} \ldots x_{n}\right)^{\theta-1} \\
& +\frac{2 \theta^{n+1} \operatorname{arctg}(y)}{\pi^{2}\left(1+y^{2}\right)} x_{1}^{2 \theta-1}\left(x_{2} \ldots x_{n}\right)^{2 \theta-1} \\
p_{\theta}\left(x_{1}, y\right)= & \frac{\theta}{\pi\left(1+y^{2}\right)}\left[1-\frac{\theta}{\pi} \operatorname{arctg}(y)\right] x_{1}^{\theta-1}+\frac{2 \theta^{2} \operatorname{arctg}(y)}{\pi^{2}\left(1+y^{2}\right) 2^{n-2}} x_{1}^{2 \theta-1} \\
= & \frac{\theta x_{1}^{\theta-1}}{\pi\left(1+y^{2}\right)}\left\{1+h\left(x_{1}, y\right)\right\} \\
h\left(x_{1}, y\right):= & \frac{\theta \operatorname{arctg}(y)}{\pi 2^{n-2}}\left[x_{1}^{\theta}-2^{n-2}\right]
\end{aligned}
$$

Como $p_{\theta}\left(x_{1}, y\right) \propto \frac{x_{1}^{\theta-1}}{\left(1+y^{2}\right)}\left\{1+h\left(x_{1}, y\right)\right\}$ e para $x_{1}<x_{1}^{\prime}, \quad y<y^{\prime}$

$$
\begin{aligned}
\text { (a) } h\left(x_{1}^{\prime}, y^{\prime}\right) h\left(x_{1}, y\right) & =h\left(x_{1}^{\prime}, y\right) h\left(x_{1}, y^{\prime}\right) \\
\text { (b) } h\left(x_{1}^{\prime}, y^{\prime}\right)+h\left(x_{1}, y\right) & \geq h\left(x_{1}^{\prime}, y\right)+h\left(x_{1}, y^{\prime}\right),
\end{aligned}
$$

então, $p_{\theta}\left(x_{1}, y\right)$ é $T P_{2}$.

(a) é evidente, enquanto (b) é válida se, e somente se,

$$
\left[\left(x_{1}^{\prime}\right)^{\theta}-2^{n-2}\right]\left\{\operatorname{arctg}\left(y^{\prime}\right)-\operatorname{arctg}(y)\right\} \geq\left[\left(x_{1}\right)^{\theta}-2^{n-2}\right]\left\{\operatorname{arctg}\left(y^{\prime}\right)-\operatorname{arctg}(y)\right\}
$$

e como $x_{1}<x_{1}^{\prime} \Rightarrow\left(x_{1}\right)^{\theta}-2^{n-2}<\left(x_{1}^{\prime}\right)^{\theta}-2^{n-2}$ enquanto que $y^{\prime}>y \Rightarrow \operatorname{arctg}\left(y^{\prime}\right)-$ $\operatorname{arctg}(y) \geq 0$, e finalmente,

$$
\left[\left(x_{1}^{\prime}\right)^{\theta}-2^{n-2}\right]\left\{\operatorname{arctg}\left(y^{\prime}\right)-\operatorname{arctg}(y)\right\} \geq\left[\left(x_{1}\right)^{\theta}-2^{n-2}\right]\left\{\operatorname{arctg}\left(y^{\prime}\right)-\operatorname{arctg}(y)\right\} .
$$

Como conseqüência,

$$
X_{i}, Y \text { são } P A\left(T P_{2}\right) i=1, \cdots, n \text {. }
$$

A seguir, veremos que $T$ e $Y$ são $P A\left(T P_{2}\right)$. 
A densidade de probabilidade para o par $(T, Y)$ é dada por

$$
g_{\theta}^{\prime}(t, y)=\frac{g_{\theta}(t, y)}{\theta^{n-1} \pi}
$$

claramente $g_{\theta}^{\prime}(t, y) \propto g_{\theta}(t, y)$ e portanto $g_{\theta}^{\prime}(t, y)$ é $T P_{2}$ se, e somente se, $g_{\theta}(t, y)$ é $T P_{2}$,

$$
g_{\theta}(t, y) \propto \frac{t^{\theta-1}}{\left(1+y^{2}\right)}\{1+k(t, y)\}
$$

onde $k(t, y):=\frac{\theta}{\pi}\left(2 t^{\theta}-1\right) \operatorname{arctg}(y)$. Como para $t^{\prime}>t, y^{\prime}>y, k$ verifica

$$
\begin{aligned}
\left(a^{\prime}\right) k\left(t^{\prime}, y^{\prime}\right) k(t, y) & =k\left(t^{\prime}, y\right) k\left(t, y^{\prime}\right) \\
\left(b^{\prime}\right) k\left(t^{\prime}, y^{\prime}\right)+k(t, y) & \geq k\left(t^{\prime}, y\right)+m k\left(t, y^{\prime}\right)
\end{aligned}
$$

então $g_{\theta}$ é $T P_{2}$.

$\left(a^{\prime}\right)$ é direta, enquanto $\left(b^{\prime}\right)$ é conseqüência da validade da seguinte desigualdade

$$
\left(2\left(t^{\prime}\right)^{\theta}-1\right)\left\{\operatorname{arctg}\left(y^{\prime}\right)-\operatorname{arctg}(y)\right\} \geq\left(2 t^{\theta}-1\right)\left\{\operatorname{arctg}\left(y^{\prime}\right)-\operatorname{arctg}(y)\right\} .
$$

Finalmente,

$$
T, Y \text { são } P A\left(T P_{2}\right)
$$

A seguir, vamos calcular as medidas $\tau_{X_{i}, Y}$ e $\tau_{T, Y}$.

$$
\begin{aligned}
p_{\theta}\left(x_{i}, y\right) & \propto \frac{x_{i}^{\theta-1}}{\left(1+y^{2}\right)}\left\{1+h\left(x_{i}, y\right)\right\} \text { portanto } \\
F_{\theta}\left(x_{i}, y\right) & \propto \int_{-\infty}^{y} \int_{0}^{x_{i}} \frac{x^{\theta-1}}{1+z^{2}} d x d z+\int_{-\infty}^{y} \int_{0}^{x_{i}} \frac{x^{\theta-1} h(x, z)}{1+z^{2}} d x d z \\
\int_{-\infty}^{y} \int_{0}^{x_{i}} \frac{x^{\theta-1}}{1+z^{2}} d x d z & =\frac{x_{i}^{\theta}}{\theta}\left\{\operatorname{arctg}(y)+\frac{\pi}{2}\right\}
\end{aligned}
$$

para os calculos restantes, é preciso lembrar que

$$
\int \frac{\operatorname{arctg}^{n}(u)}{1+u^{2}} d u=\frac{\operatorname{arctg}^{n+1}(u)}{n+1}, \text { subst. } v=\operatorname{arctg}(u), d v=\frac{d u}{1+u^{2}}
$$




$$
\begin{aligned}
\int_{-\infty}^{y} \int_{0}^{x_{i}} \frac{x^{\theta-1} h(x, z)}{1+z^{2}} d x d z & =\frac{\theta}{\pi 2^{n-2}} \int_{-\infty}^{y} \int_{0}^{x_{i}} x^{2 \theta-1} \frac{\operatorname{arctg}(z)}{1+z^{2}} d x d z- \\
& =\left\{\int_{-\infty}^{y} \int_{0}^{x_{i}} x^{\theta-1} \frac{\operatorname{arctg}(z)}{1+z^{2}} d x d z-\frac{\pi^{2}}{4}\right\}\left[\frac{x_{i}^{2 \theta}}{\pi 2^{n}}-\frac{x_{i}^{\theta}}{2 \pi}\right] \\
F_{\theta}\left(x_{i}, y\right) & =\frac{\theta}{\pi}\{I+I I\} \\
I:= & \frac{x_{i}^{\theta}}{\theta}\left\{\operatorname{arctg}^{2}(y)+\frac{\pi}{2}\right\} \\
I I:= & \left\{\operatorname{arctg}^{2}(y)-\frac{\pi^{2}}{4}\right\}\left[\frac{x_{i}^{2 \theta}}{\pi 2^{n}}-\frac{x_{i}^{\theta}}{2 \pi}\right]
\end{aligned}
$$

logo,

$$
\begin{aligned}
& F_{\theta}\left(x_{i}, y\right) p_{\theta}\left(x_{i}, y\right)=\frac{\theta}{\pi}\left\{I \cdot p_{\theta}\left(x_{i}, y\right)+I I \cdot p_{\theta}\left(x_{i}, y\right)\right\} \\
& I \cdot p_{\theta}\left(x_{i}, y\right)=\frac{x_{i}^{2 \theta-1} \theta}{\pi\left(1+y^{2}\right) 2 \pi}\left\{\frac{\pi(2-\theta)}{\theta} \operatorname{arctg}(y)-2 \operatorname{arctg}^{2}(y)+\frac{\pi}{2^{n-2}} \operatorname{arctg}(y) x_{i}^{\theta}\right. \\
& \left.+\frac{1}{2^{n-3}} \operatorname{arctg}^{2}(y) x_{i}^{\theta}+\frac{\pi^{2}}{\theta}\right\} \\
& I I \cdot p_{\theta}\left(x_{i}, y\right)=\frac{x_{i}^{2 \theta-1} \theta}{\pi\left(1+y^{2}\right) 2 \pi}\left\{-\frac{\theta \pi}{4} \operatorname{arctg}(y)-\operatorname{arctg}^{2}(y)+\frac{\theta}{\pi} \operatorname{arctg}^{3}(y)\right. \\
& +\frac{3 \theta \pi}{2^{n+1}} \operatorname{arctg}(y) x_{i}^{\theta}+\frac{1}{2^{n-1}} \operatorname{arctg}^{2}(y) x_{i}^{\theta}-\frac{3 \theta}{2^{n-1} \pi} \operatorname{arctg}^{3}(y) x_{i}^{\theta} \\
& \left.-\frac{\theta \pi}{2^{2 n-1}} \operatorname{arctg}(y) x_{i}^{2 \theta}+\frac{\theta}{\pi 2^{2 n-3}} \operatorname{arctg}^{3}(y) x_{i}^{2 \theta}-\frac{\pi^{2}}{2^{n+1}} x_{i}^{\theta}+\frac{\pi^{2}}{4}\right\} \\
& F_{\theta}\left(x_{i}, y\right) p_{\theta}\left(x_{i}, y\right)=\frac{\theta}{\pi}\left\{I \cdot p_{\theta}\left(x_{i}, y\right)+I I \cdot p_{\theta}\left(x_{i}, y\right)\right\} \\
& \frac{x_{i}^{2 \theta-1} \theta^{2}}{2 \pi^{3}\left(1+y^{2}\right)}\left\{\left(\frac{2}{\theta}-1-\frac{\theta}{4}\right) \operatorname{arctg}(y)-3 \operatorname{arctg}^{2}(y)\right. \\
& +\frac{\theta}{\pi} \operatorname{arctg}^{3}(y)+\frac{\left(3 \theta+2^{3}\right) \pi}{2^{n+1}} \operatorname{arctg}(y) x_{i}^{\theta}+\frac{5}{2^{n-1}} \operatorname{arctg}^{2}(y) x_{i}^{\theta} \\
& -\frac{3 \theta}{\pi 2^{n-1}} \operatorname{arctg}^{3}(y) x_{i}^{\theta}-\frac{\theta \pi}{2^{2 n-1}} \operatorname{arctg}(y) x_{i}^{2 \theta} \\
& \left.+\frac{\theta}{\pi 2^{2 n-3}} \operatorname{arctg}^{3}(y) x_{i}^{2 \theta}-\frac{\pi^{2}}{2^{n+1}} x_{i}^{\theta}+\frac{\theta+4}{4 \theta} \pi^{2}\right\} \text {. }
\end{aligned}
$$


Como

$$
\int_{-\infty}^{+\infty} \frac{\operatorname{arctg}^{n}(u)}{1+u^{2}} d u=0, \text { se } n \text { é impar }
$$

então,

$$
\begin{aligned}
\int_{-\infty}^{+\infty} \int_{0}^{1} F_{\theta}\left(x_{i}, y\right) p_{\theta}\left(x_{i}, y\right) d x_{i} d y & =\int_{-\infty}^{+\infty} \int_{0}^{1} \frac{\theta}{\pi}\left\{I \cdot p_{\theta}\left(x_{i}, y\right)+I I \cdot p_{\theta}\left(x_{i}, y\right)\right\} d x_{i} d y \\
& =-I I I+I V-V+V I \\
I I I & :=c_{2} \frac{1}{2 \theta} \int_{-\infty}^{+\infty} \frac{\operatorname{arctg}^{2}(y)}{1+y^{2}} d y, c_{2}=\frac{3 \theta^{2}}{2 \pi^{3}} \\
& =\frac{\theta}{16} \\
I V & :=c_{5} \frac{1}{3 \theta} \int_{-\infty}^{+\infty} \frac{\operatorname{arctg}^{2}(y)}{1+y^{2}} d y, c_{5}=\frac{5 \theta^{2}}{2^{n} \pi^{3}} \\
& =\frac{5 \theta}{3^{2} \cdot 2^{n+2}} \\
V & :=c_{9} \frac{1}{3 \theta} \int_{-\infty}^{+\infty} \frac{1}{1+y^{2}} d y, c_{9}=\frac{\theta^{2}}{2^{n+2} \pi} \\
& =\frac{\theta}{3.2^{n+2}} \\
V I & :=c_{10} \frac{1}{2 \theta} \int_{-\infty}^{+\infty} \frac{1}{1+y^{2}} d y, c_{10}=\frac{\theta(\theta+4)}{8 \pi} \\
& =\frac{\theta+4}{16} .
\end{aligned}
$$

Logo

$$
\int_{-\infty}^{+\infty} \int_{0}^{1} F_{\theta}\left(x_{i}, y\right) p_{\theta}\left(x_{i}, y\right) d x_{i} d y=\frac{\theta}{3^{2} \cdot 2^{n+1}}+\frac{1}{4}
$$

então,

$$
\begin{aligned}
\tau_{X_{i}, Y} & =4 \int_{-\infty}^{+\infty} \int_{0}^{1} F_{\theta}\left(x_{i}, y\right) p_{\theta}\left(x_{i}, y\right) d x_{i} d y-1 \\
& =\frac{\theta}{3^{2} \cdot 2^{n-1}}, i=1, \cdots, n
\end{aligned}
$$

Notemos que naturalmente $\tau_{X_{i}, Y} \geq 0$. 
A seguir a medida $\tau_{T, Y}$,

$$
\begin{gathered}
g_{\theta}^{\prime}(t, y)=\frac{\theta t^{\theta-1}}{\pi\left(1+y^{2}\right)}+\frac{2 \theta^{2}}{\pi^{2}} t^{2 \theta-1} \frac{\operatorname{arctg}(y)}{\left(1+y^{2}\right)}-\frac{\theta^{2}}{\pi^{2}} t^{\theta-1} \frac{\operatorname{arctg}(y)}{\left(1+y^{2}\right)} \\
G_{\theta}^{\prime}(t, y)=\left(\frac{\theta}{8}+\frac{1}{2}\right) t^{\theta}-\frac{\theta}{8} t^{2 \theta}+\frac{1}{\pi} t^{\theta} \operatorname{arctg}(y)-\frac{\theta}{2 \pi^{2}} t^{\theta} \operatorname{arctg}^{2}(y)+\frac{\theta}{2 \pi^{2}} t^{2 \theta} \operatorname{arctg}^{2}(y) \\
\int_{-\infty}^{+\infty} \int_{0}^{1} G_{\theta}^{\prime}(t, y) g_{\theta}^{\prime}(t, y)=A_{1} \frac{\pi}{2 \theta}-A_{2} \frac{\pi}{3 \theta}-A_{3} \frac{\pi^{3}}{24 \theta}+A_{4} \frac{\pi^{3}}{36 \theta}+A_{5} \frac{\pi^{3}}{36 \theta}-A_{6} \frac{\pi^{3}}{24 \theta} \\
A_{1}=\frac{\theta}{\pi}\left(\frac{\theta}{8}+\frac{1}{2}\right), A_{2}=\frac{\theta^{2}}{8 \pi}, A_{3}=\frac{\theta^{2}}{2 \pi^{3}} \\
A_{4}=\frac{\theta^{2}}{2 \pi^{3}}, A_{5}=\frac{2 \theta^{2}}{\pi^{3}}, A_{6}=\frac{\theta^{2}}{\pi^{3}}
\end{gathered}
$$

finalmente,

$$
\int_{-\infty}^{+\infty} \int_{0}^{1} G_{\theta}^{\prime}(t, y) g_{\theta}^{\prime}(t, y)=\frac{\theta}{36}+\frac{1}{4}
$$

Logo,

$$
\tau_{T, Y}=\frac{\theta}{3^{2}}
$$

Assim,

$$
\tau_{X_{i}, Y}=\tau_{T, Y} \frac{1}{2^{n-1}}
$$

e como $\frac{1}{2^{n-1}} \leq 1 \Rightarrow \frac{\theta}{9} \frac{1}{2^{n-1}} \leq \frac{\theta}{9}$, pois $0<\theta<1$, finalmente,

$$
0<\tau_{X_{i}, Y} \leq \tau_{T, Y}, \quad i=1, \cdots, n
$$

Retomando: para $0<\theta<1,0 \leq x_{i} \leq 1, i=1,2,-\infty<y<+\infty$. Consideremos $\left(X_{1}, \cdots, X_{n}\right)$ como a base da predição e $Y$ a variável aleatória a predizer.

$$
\begin{aligned}
& F_{X_{1}, \cdots, X_{n}, Y}\left(x_{1}, \cdots, x_{n}, y\right)= \\
& \quad \frac{x_{1}^{\theta} \ldots x_{n}^{\theta}}{2}+\frac{\operatorname{arctg}(y)}{\pi} x_{1}^{\theta} \ldots x_{n}^{\theta}+\frac{\theta}{2 \pi^{2}}\left\{\operatorname{arctg}^{2}(y)-\frac{\pi^{2}}{2^{2}}\right\}\left\{\frac{x_{1}^{2 \theta} \ldots x_{n}^{2 \theta}}{2^{n-1}}-x_{1}^{\theta} \ldots x_{n}^{\theta}\right\},
\end{aligned}
$$


observemos que $F_{X_{1}, \cdots, X_{n}, Y}$ é contínua e

$$
F_{X_{1}, \cdots, X_{n}}\left(x_{1}, \cdots, x_{n}\right)=x_{1}^{\theta} \ldots x_{n}^{\theta}, \text { contínua em }[0,1]
$$

Sugerimos, então como variável preditora de $Y$ a $F_{X_{1} \cdots, X_{n}}\left(X_{1}, \cdots, X_{n}\right)=X_{1}^{\theta} \ldots X_{n}^{\theta}$. É possível calcular a medida $\tau$ de Kendall entre $F_{X_{1}, \cdots, X_{n}}\left(X_{1}, \cdots, X_{n}\right)$ e $Y$. Como a medida $\tau$ é invariante perante funções crescentes, e $F_{X_{1}, \cdots, X_{n}}\left(X_{1}, \cdots, X_{n}\right)=T^{\theta}$, resulta

$$
\tau_{F_{X_{1}, \cdots, X_{n}}\left(X_{1}, \cdots, X_{n}\right), Y}=\tau_{T, Y}=\frac{\theta}{3^{2}} .
$$

E podemos notar que

$$
\tau_{F_{X_{1}, \cdots, X_{n}}\left(X_{1}, \cdots, X_{n}\right), Y} \geq \tau_{X_{i}, Y}, \quad i=1, \cdots, n .
$$




\subsection{Predição Empírica}

5.3.1 Simulação: A seguir, descreveremos a simulação. Temos presente que, pelos estudos baseados no conhecimento das virtudes da medida $\tau$ de Kendall, ela pode "condensar" a informação da concordância versus discordância entre pares de variáveis aleatórias. Quando, nas seções prévias, trabalhamos com a distribuição acumulada e suas peculiaridades, tínhamos em mente varios tópicos. Em primeiro lugar, é sabido que a distribuição dos dados é toda a informação de que precisamos para poder fazer inferência sobre os dados; em segundo lugar, se tivermos três variáveis aleatórias $U_{1}, U_{2}, U_{3}$, onde $U_{1}, U_{i}$ são $P A\left(T P_{2}\right), i=2,3$, e $F_{U_{2}, U_{3}}(\cdot, \cdot)$ é a distribuição acumulada de $\left(U_{2}, U_{3}\right)$, a variável aleatória $U:=F_{U_{2}, U_{3}}\left(U_{2}, U_{3}\right)$ é tal que $\tau_{U_{1}, U} \geq \tau_{U_{1}, U_{i}}, \quad i=2,3$, prévia hipótese de concordância entre $U_{2}$ e $U_{3}$.

Suponhamos agora que $U_{2}$ e $U_{3}$ são resultados de análises clínicas feitas em um paciente e $U_{1}$ representa o diagnóstico elaborado com base em $U_{2}$ e $U_{3}$. Suponhamos, ainda, que dispomos de um banco de dados com $N-1$ dessas triplas: $\left\{u_{i 1}, u_{i 2}, u_{i 3}\right\}_{i=1}^{N-1}$; em seguida, incorporamos um novo paciente com estudos clínicos $\left(u_{N 2}, u_{N 3}\right)$. Estamos interesados em fazer uma predição para o diagnóstico desse novo paciente: $u_{N 1}$. Para isso, pretendemos utilizar a distribuição acumulada de $U_{2}$ e $U_{3}$ (que supostamente bastavam para concluir $U_{1}$ ) como preditor semente (preditor de variabilidade) do diagnóstico $U_{1}$. Esse preditor tem imagem em $[0,1]$, enquanto $U_{1}$ pode assumir valores fora desse espaço; por isso, precisamos utilizar, para a predição de $u_{N 1}$, a informação fornecida pelo histórico de $U_{1}$, ou seja, $\left\{u_{i 1}\right\}_{i=1}^{N-1}$, ajustando o preditor semente. Esperamos que o preditor semente reproduza o comportamento de $U_{1}$ salvo escala e deslocamento. 
Diversos caminhos poderiam ser utilizados para elaborar preditores sementes, como considerar a distribuição acumulada dos ternos até $N-1$ e "combinar" esse novo preditor com o descrito anteriormente.

\subsubsection{Discussão e análise}

Na figura 5.3.3, a distribuição acumulada das variáveis $U_{2}$ e $U_{3}$ serve para predizer o comportamento da variável "oculta" $U_{1}$. Esse preditor de comportamento pretende representar todo o campo de chegada da função $U_{1},(-\infty,+\infty)$, no intervalo $(0,1)$, sofrendo alterações somente quando $U_{1}$ sofrer alterações. Vale destacar que a distribuição acumulada de $\left(U_{2}, U_{3}\right)$ tem informação a respeito da função $U_{1}$. Por isso esse preditor do comportamento reflete as mudanças ocorridas em $U_{1}$. A partir da existência da conexão entre as três variáveis, pela normal tri-variada (não independentes), é possível afirmar que as variáveis $U_{2}$ e $U_{3}$ têm "algo" a dizer sobre o comportamento da variável $U_{1}$. Isto será alcançado pela função de distribuição acumulada do par $\left(U_{2}, U_{3}\right)$.

Na figura 5.3.4, o preditor do comportamento, alocado entre 0 e 1 , é corrigido pela média e a variância histórica da variável $U_{1}$, calculadas a partir dos valores da amostra. Desse modo, é incorporada a informação passada da variável $U_{1}$ na fabricação do preditor de cada novo valor possível da variável $U_{1}$. Essa proposta, de preditor, permite aproveitar a distribuição acumulada, como preditor de comportamento. ED e $V D$ representam, respectivamente, o valor esperado e a variância da diferença entre o preditor Pred e o valor verdadeiro ValorVerd. 
Uma outra proposta apresentada aqui consiste em: suponhamos que as triplas históricas (do banco de dados) $\left(u_{i 1}, u_{i 2}, u_{i 3}\right)_{i=1}^{N-1}$ são ordenadas pela norma euclideana calculada para as duas últimas componentes das triplas, $\left\{\left(u_{i 2}, u_{i 3}\right)\right\}_{i=1}^{N-1}$, na ordem crescente. Suponhamos, ainda, que un novo par de valores $\left(u_{N 2}, u_{N 3}\right)$ é recebido e alocado segundo a norma euclideana, sem perda da generalidade, como maior que $\left(u_{N-12}, u_{N-13}\right)$. Então, o preditor proposto para o valor desconhecido $u_{N 1}$ será uma combinação entre os valores da acumulada de $\left(u_{N-11}, u_{N-12}, u_{N-13}\right)$ e da acumulada dos valores revelados $\left(u_{N 2}, u_{N 3}\right)$, tal combinação, vai ser ajustada pela média e a variância do histórico da variável $U_{1}$, i.e., $\left\{u_{i 1}\right\}_{i=1}^{N-1}$.

A idéia é a seguinte: o ponto $\left(u_{N-11}, u_{N-12}, u_{N-13}\right)$ é o ponto mais próximo de $\left(u_{N 1}, u_{N 2}, u_{N 3}\right)$, em toda a amostra e considerando a norma euclideana, das duas últimas componentes. Portanto, esse ponto $\left(u_{N-11}, u_{N-12} \cdot u_{N-13}\right)$ aporta "informação" de $u_{N 1}$, através da acumulada associada a ele. O par $\left(u_{N 2}, u_{N 3}\right)$ revelado prediz o comportamento de $u_{N 1}$, como já induzimos, mediante a distribuição acumulada conjunta.

Logo, aquelas sementes - acumulada conjunta de $\left(u_{N 2}, u_{N 3}\right)$ e acumulada conjunta de $\left(u_{N-11}, u_{N-12}, u_{N-13}\right)$ - serão combinadas com os valores da média e variância amostral, fornecidas por $\left\{u_{i 1}\right\}_{i=1}^{N-1}$, vide figuras 5.3.7-5.3.8.

Vale destacar, que em nossa simulação, a "melhor" proposta foi a primeira, segundo os cálculos feitos sobre as médias das diferenças. Pois, sendo "Pred" o primeiro preditor e "Eva" o segundo, temos que

$$
E(\text { ValorVerd }- \text { Pred })=.1072<E(\text { ValorVerd }- \text { Eva })=.3317
$$


onde $E$ denota o valor esperado e ValorVerd. denota a variável $U_{1}$, revelada. Notemos que o segundo preditor, Eva, apresenta uma outra opção de incorporação da informação passada, da variável $U_{1}$.

Planejamos continuar com a pesquisa destas propostas, com a idéia de implementar criterios para "decidir" qual preditor pode ser mais indicado segundo o problema abordado. Acreditamos que a idéia possa ser fortalecida com a incorporação da natureza dos dados. Certamente, a estrutura e origem dos dados poderá orientar a escolha dos ajustes, fatores de escala e deslocamento, utilizados para posicionar os preditores sementes.

\subsubsection{Conjecturas sobre o Processo de Predição:}

Suponhamos a seguinte situação: $U_{2}, \cdots, U_{n}$ representam análises clínicas feitas em um paciente e $U_{1}$ representa o diagnóstico respeito da presença de alguma doença. Suponhamos, ainda, que possuímos um banco de dados no qual constam os registros de $m$ pacientes. Assim $\left\{\left(u_{i 1}, u_{i 2}, \cdots, u_{i n}\right)\right\}_{i=1}^{m}$ representa o banco de dados. Para o paciente $i_{0^{-}}$ésimo cujas análises registradas foram $\left(u_{i_{0} 2}, \cdots, u_{i_{0} n}\right)$, o diagnóstico registrado foi $u_{i_{0} 1}$. Nosso objetivo é: admitido um novo paciente, de rótulo $m+1$, com análises clínicas $\left(u_{(m+1) 2}, \cdots, u_{(m+1) n}\right)$, fornecer uma predição do diagnóstico $u_{(m+1) 1}$, que é completamente desconhecido. As ferramentas à nossa disposição são basicamente: o banco de dados registrados e a nova $(n-1)$-upla de análises do indivíduo $m+1$. Portanto, pretendemos fazer inferência - predição - referente ao estado futuro do paciente - isto é, captar os elementos contidos nas análises clínicas do presente estado do paciente, que induzem um estado futuro. Naturalmente, na 
formulação prévia é esperado que exista um comportamento dos dados trabalhados, a saber, refere-se a três "afirmações" fundamentais:

1. A seqüência de análises observada - que foi determinada por especialistas com conhecimento profundo do problema - é suficiente para fazer inferência respeito do estado futuro do paciente. Acredita-se que a partir das análises observadas é possível determinar o diagnóstico. Ou seja que o conjunto de dados disponíveis sobre cada paciente contém a essência da "informação" que é necessária para fazer inferência sobre o estado futuro do paciente.

2. A inferência do diagnóstico para um novo paciente $m+1$ só poderá ser realizada se suas análises clínicas $\left(u_{(m+1) 2}, \cdots, u_{(m+1) n}\right)$ tiverem a mesma natureza dos pacientes já existentes no banco de dados, ou seja: somente se o novo paciente tiver aproximadamente as mesmas características (etária, socio-económica, etc.) dos pacientes já registrados.

3. O banco de dados e seu carácter preditivo: se for comunicada uma nova $n-1$ upla $\left(u_{(m+1) 2}, \cdots, u_{(m+1) n}\right)$ referente ao paciente $m+1$ - e se essa $n$-1 upla estiver próxima de alguma outra $n$-1 upla $\left(u_{i_{0} 2}, \cdots, u_{i_{0} n}\right)$ associada ao paciente $i_{0}$, cujo registro $\left(u_{i_{0} 1}, u_{i_{0} 2}, \cdots, u_{i_{0} n}\right)$ esteja contido no banco de dados, então a inferência de $u_{(m+1) 1}$ obtida a partir de $\left(u_{(m+1) 2}, \cdots, u_{(m+1) n}\right)$ deverá estar próxima do valor observado em $u_{i_{0} 1}$.

O preditor a ser implementado terá as seguintes passos: primeiramente, fazemos menção à importância indiscutível da distribuição acumulada. A distribuição 
acumulada de um vetor aleatório $\left(X_{1}, \cdots, X_{k}\right)$ contabiliza as ocorrências de um evento do tipo $\left\{X_{1} \leq x_{1}, \cdots, X_{k} \leq x_{k}\right\}$. Assim, $P\left(X_{1} \leq x_{1}, \cdots, X_{k} \leq x_{k}\right)$ resume toda a informação (unidimensional), e que pode ser obtida a partir de qualquer banco de dados. Calculado através da empírica (por exemplo). Deste modo $P\left(U_{2} \leq u_{i 2}, \cdots, U_{n} \leq u_{i n}\right)$ contém toda a informação embutida na amostra para a inferência de $U_{1}$ quando ela assume o valor $u_{i 1}$, correspondente. Por ser distribuição conjunta acumulada, $P\left(U_{2} \leq u_{i 2}, \cdots, U_{n} \leq u_{\text {in }}\right)$ incorpora não apenas o quadro clínico atual do paciente $i$, mas também os possíveis estágios pelos quais este passou. Esse preditor pontual $P\left(U_{2} \leq u_{i 2}, \cdots, U_{n} \leq u_{i n}\right)$ de $U_{1}=u_{i 1}$, encontra-se alocado entre 0 e 1 , e precisa ser corrigido pelo aporte feito pelo histórico da variável $U_{1}$.

\section{Implementando o preditor:}

Quando comunicada a $n$-1 upla $\left(u_{(m+1) 2}, \cdots, u_{(m+1) n}\right)$ de análises clínicas efetuadas sobre o paciente rotulado por $m+1$, imediatamente procuramos em nosso banco de dados a $n-1$ upla que fique mais próxima da comunicada, digamos $\left(u_{i_{0} 2}, \cdots, u_{i_{0} n}\right)$. A seguir, calculamos $P\left(U_{2} \leq u_{i_{0} 2}, \cdots, U_{n} \leq u_{i_{0} n}\right)$ que é o preditor de monotonicidade do valor $u_{(m+1) 1}$ ainda desconhecido. Para definir o preditor definitivo do valor $u_{(m+1) 1}$ é preciso deslocar a quantidade $P\left(U_{2} \leq u_{i_{0} 2}, \cdots, U_{n} \leq u_{i_{0} n}\right)$ usando fatores obtidos a partir das observações de $U_{1}$, a saber: $\left\{u_{i 1}\right\}_{i=1}^{m}$. Deste modo ficou definido um preditor pontual para cada possível valor da variável $U_{1}$. 


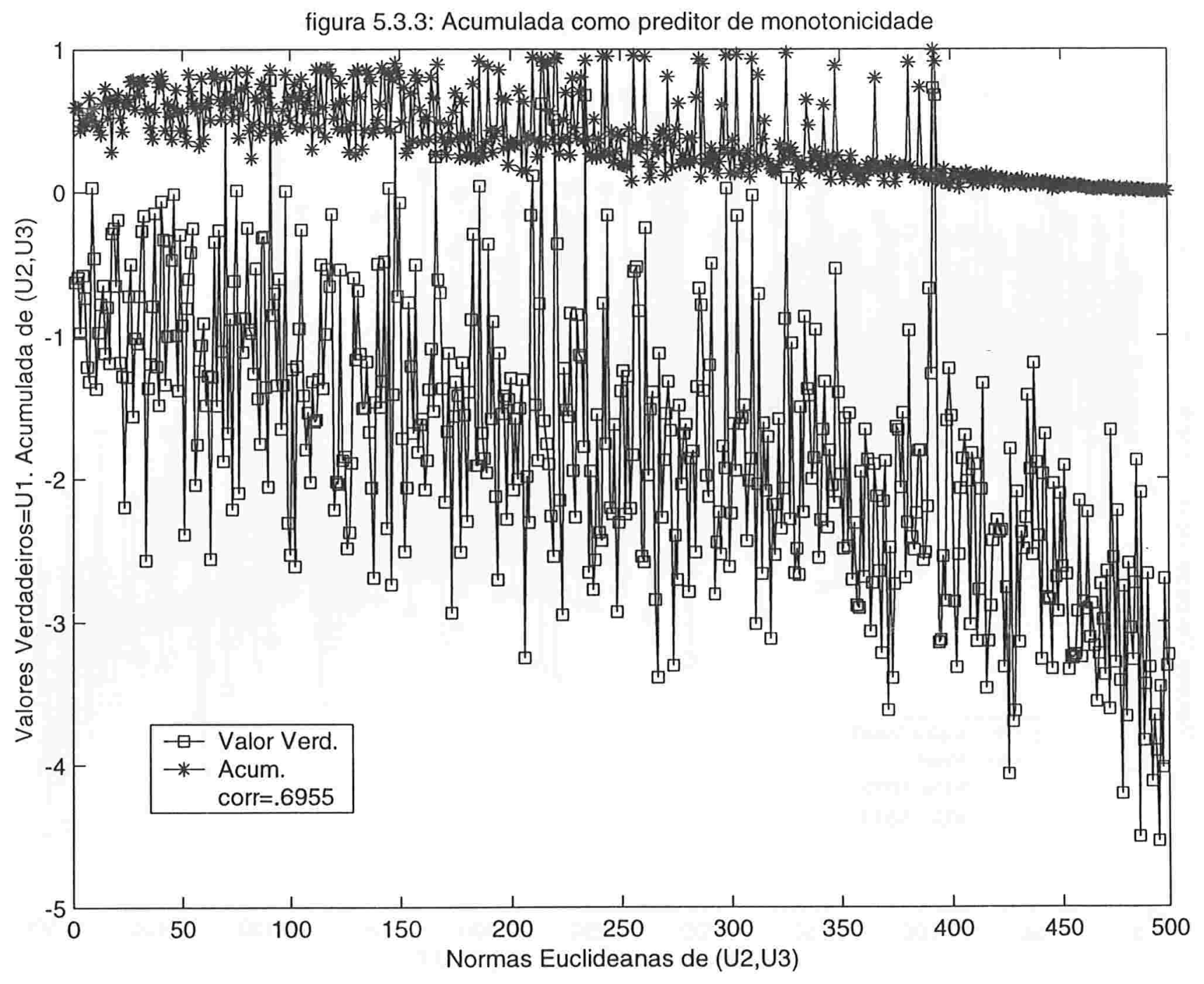




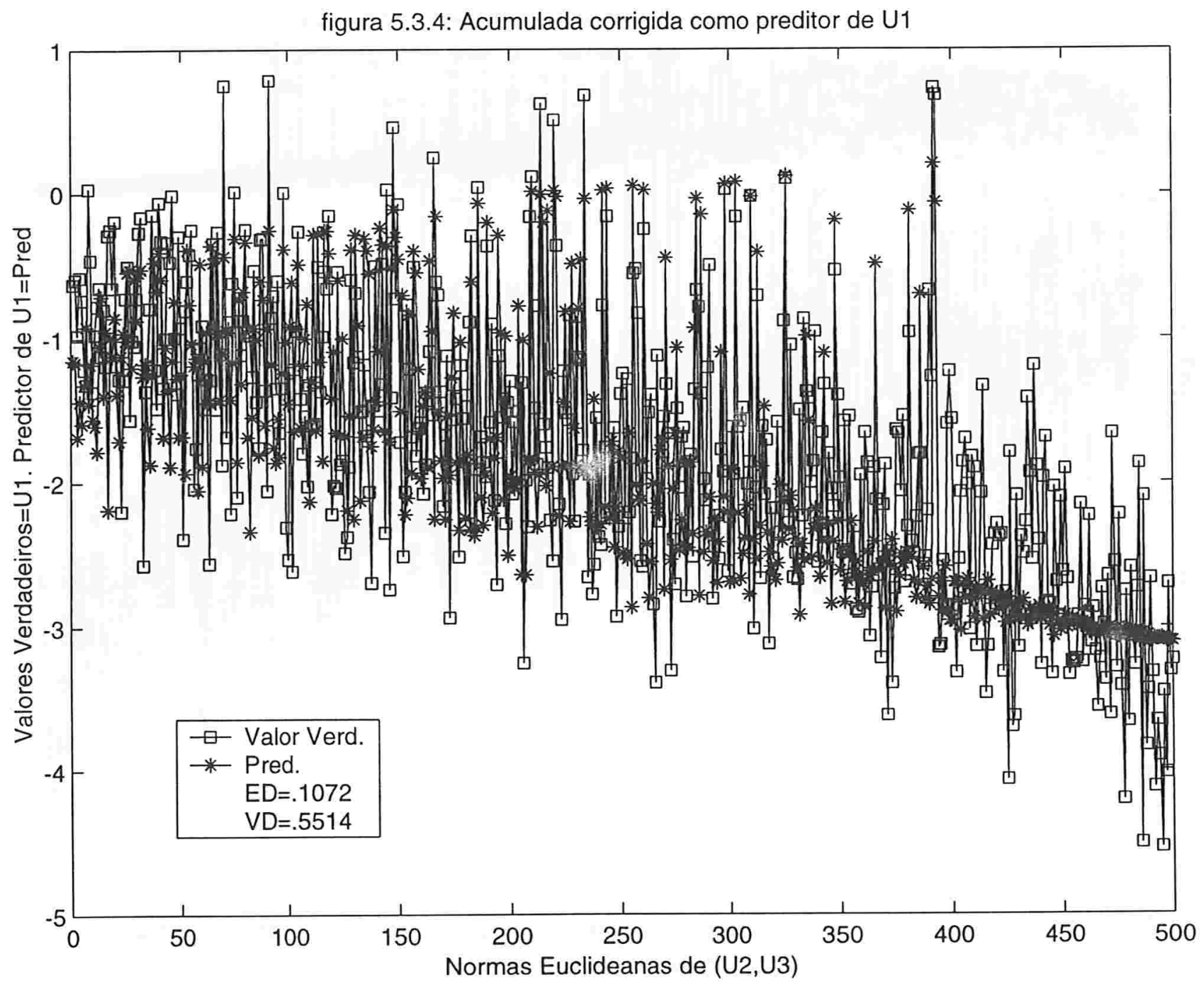


figura 5.3.5: Valores Verdadeiros $=\mathrm{U} 1$

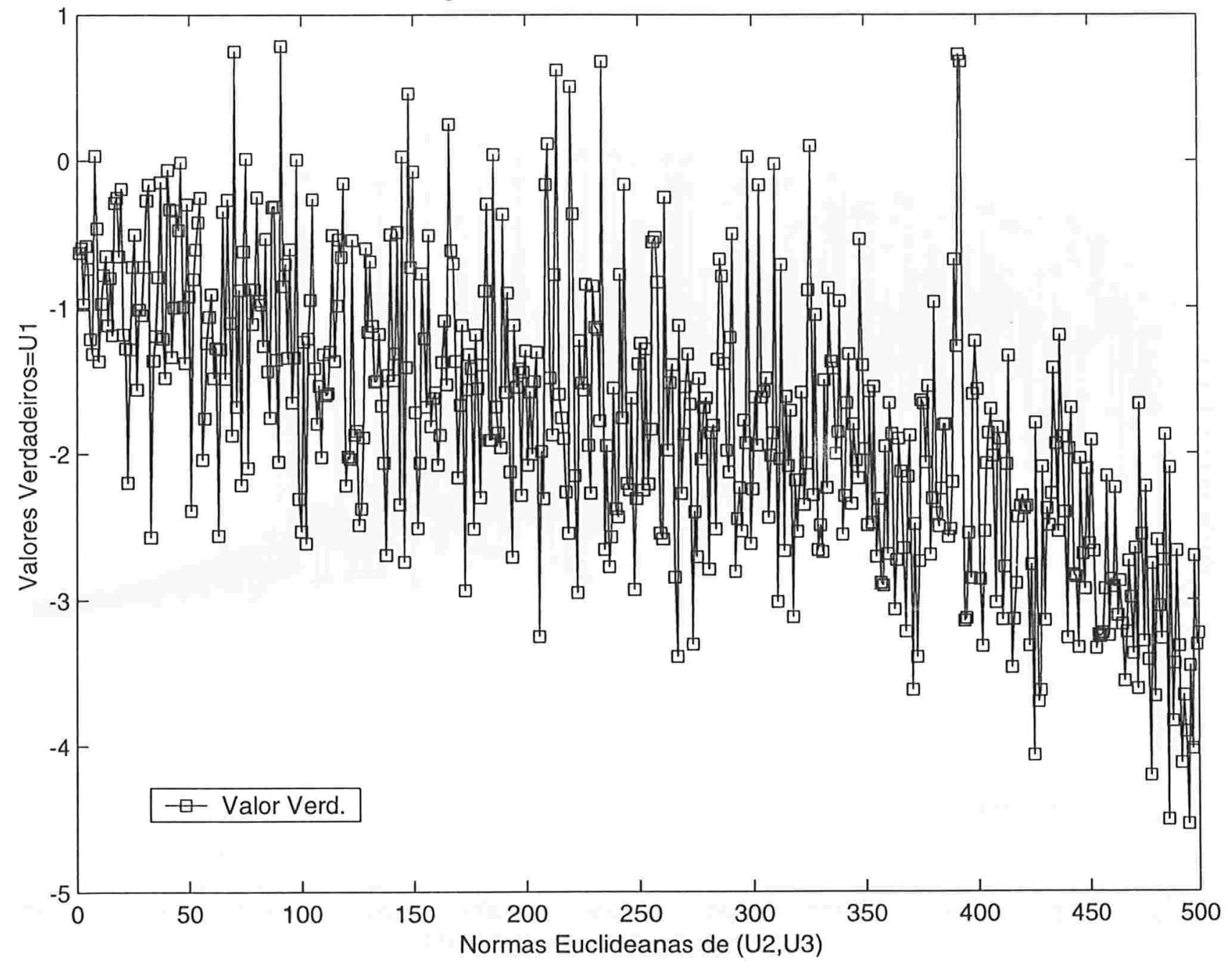




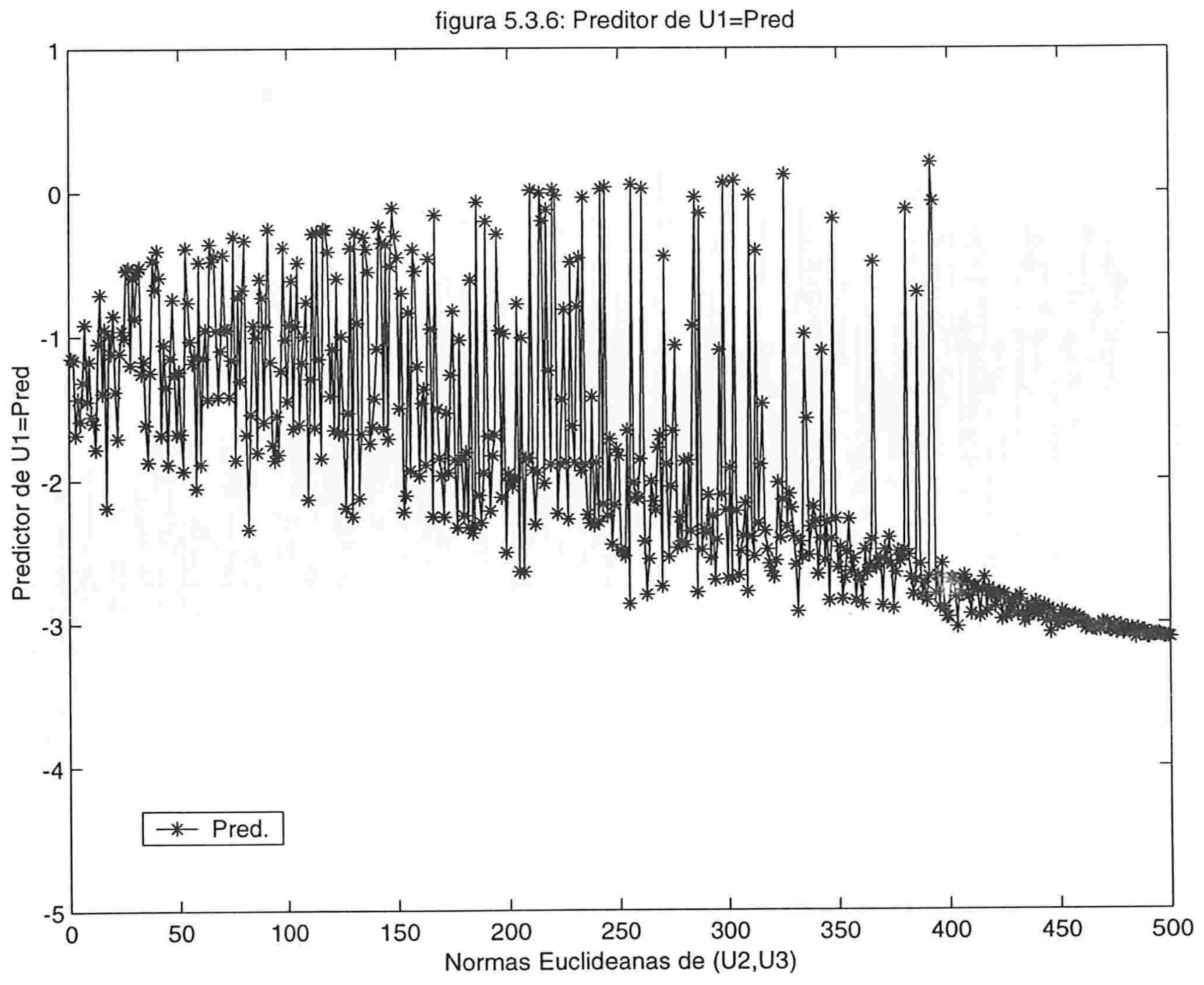




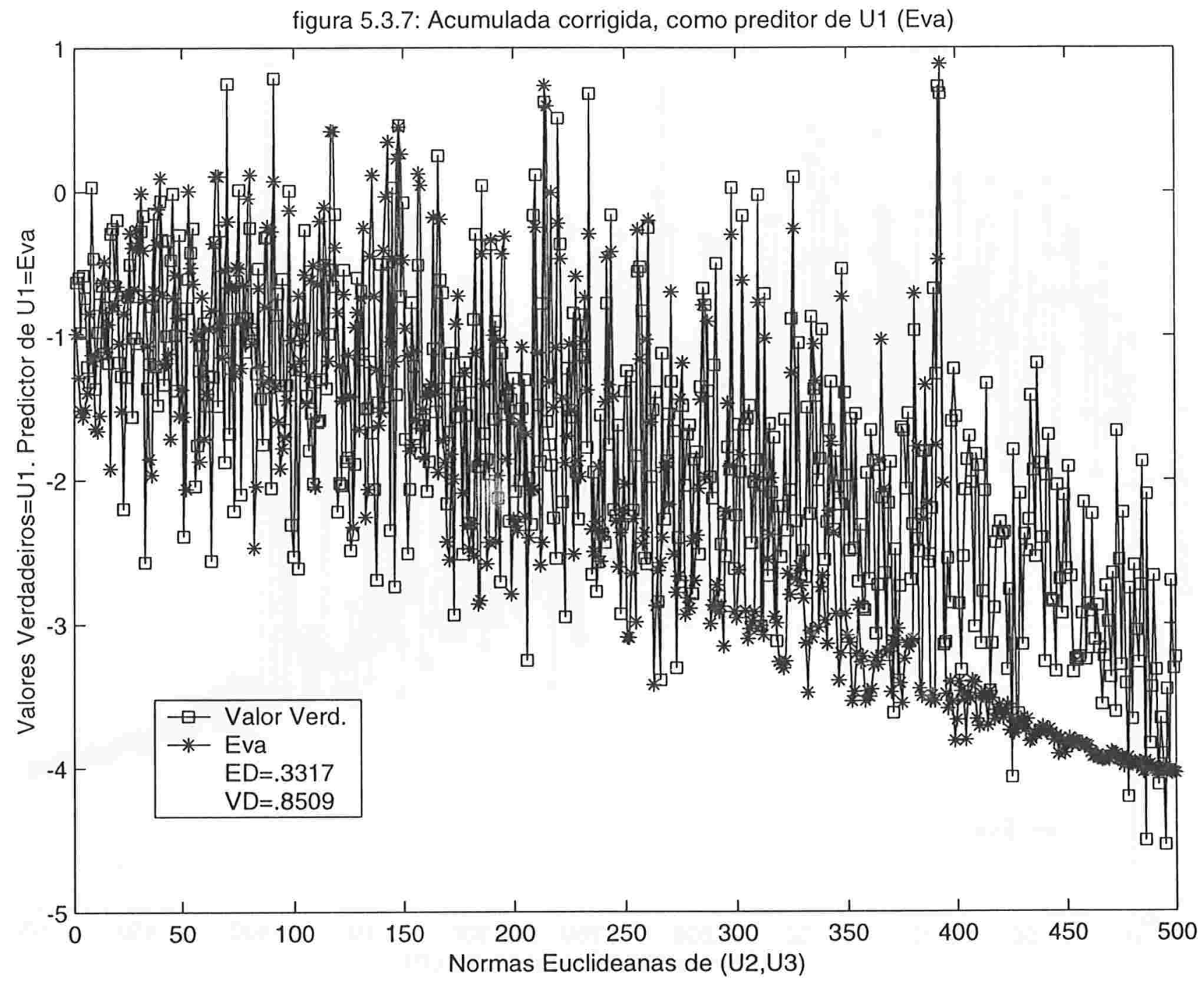




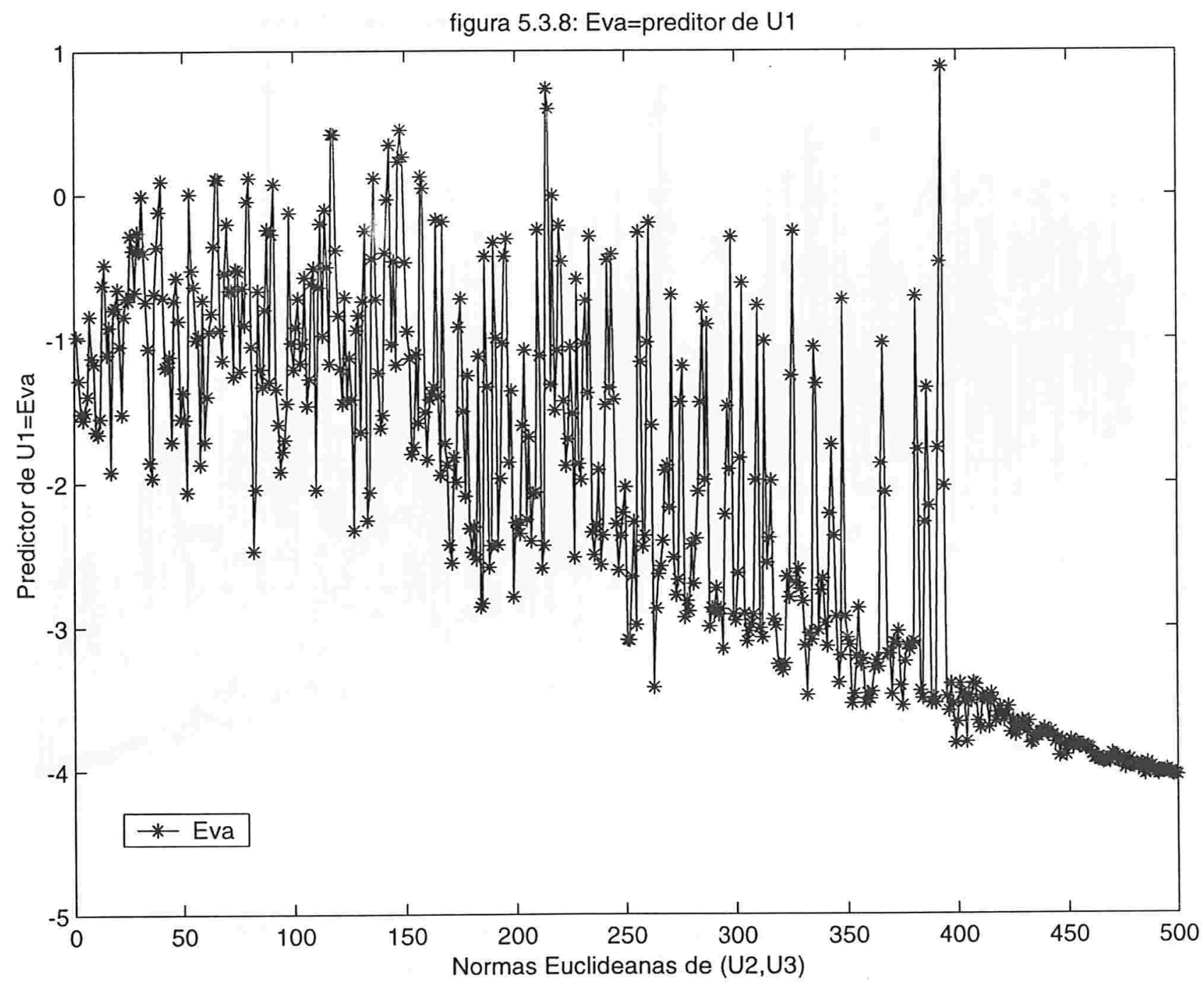




\title{
Apêndice
}

\author{
A.1. Programas \\ - Para o Cálculo de Acoplamentos e Medida $\tau$-Kendall \\ - Simulação
}

A.2. Condição $T P_{2}\left(R R_{2}\right)$ em pares, Distribuição
Conjunta e Operação Bayesiana: Prior/Posterior 


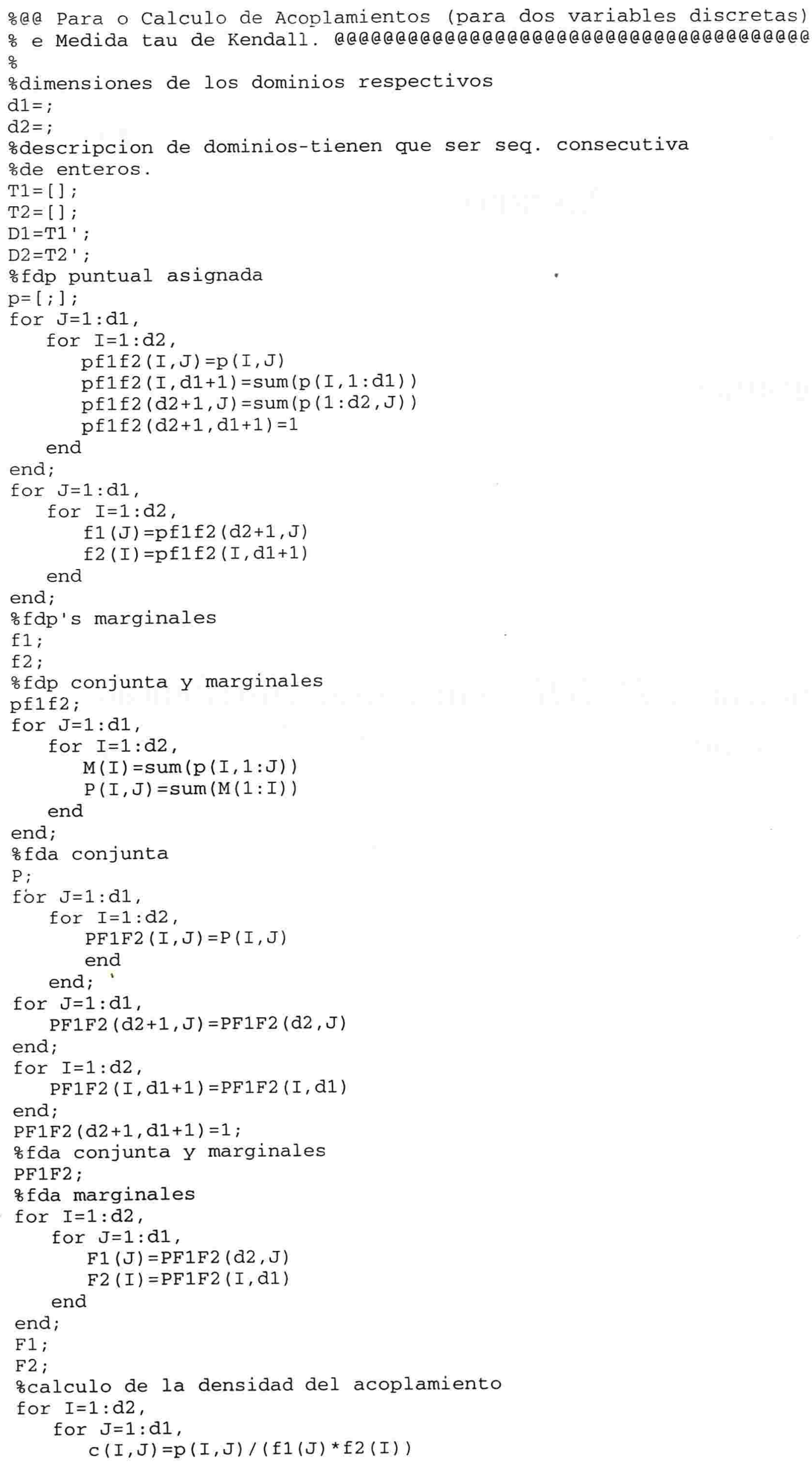


end

end;

C;

\%c representa la asignacion por intervalos, otorgada a

- la densidad del acoplamiento

\% la densidad del acoplamiento es:

$\because \mathrm{C}(\mathrm{U}, \mathrm{V})=\mathrm{C}(\mathrm{I}, \mathrm{J})$ si $\mathrm{u}$ en $[\mathrm{F} 1(\mathrm{I}-1), \mathrm{F} 1(\mathrm{I}))], \mathrm{V}$ en $[\mathrm{F} 2(\mathrm{~J}-1), \mathrm{F} 2(\mathrm{~J}))]$

for $I=1: \mathrm{d} 2$,

for $J=1: d 1$,

iP1 $(\mathrm{J})=\mathrm{F} 1(\mathrm{~J})$

i $P 2(I)=F 2(I)$

end

end;

\%dominio de definicion de la densidad del acoplamiento

iP1;

iP2;

\%a@a@a@a@a@a@a@a@a@a@a@a@a@a@a@a@a@a@a@a@a@a@a@

\% a seguir verificamos que c es acoplamiento.

\%calculo de la longitud de los intervalos de dominio de la densidad

\%del acoplamiento

L1 $(1)=$ iP1 (1);

L2 $(1)=$ iP2 $(1)$;

for $I=2: d 2$,

for $\mathrm{J}=2: \mathrm{d} 1$,

$\mathrm{L} 1(\mathrm{~J})=i \mathrm{P} 1(\mathrm{~J})-i \mathrm{P} 1(\mathrm{~J}-1)$

end

$L 2(I)=i P 2(I)-i P 2(I-1)$

end;

L1;

L2;

for $I=1: d 2$,

for $\mathrm{J}=1: \mathrm{d} 1$,

$S(I, J)=C(I, J) * L 1(J) * L 2(I)$

end

end;

s;

for $\mathrm{J}=1: \mathrm{d} 1$,

$\mathrm{Ci}(1, \mathrm{~J})=\operatorname{sum}(\mathrm{s}(1,1: \mathrm{J}))$

end

for $I=2: d 2$,

for $J=1: d 1$,

end

$\mathrm{Ci}(I, J)=\mathrm{Ci}(I-1, J)+\operatorname{sum}(\mathrm{S}(I, 1: J))$

end

\% acumulada C (a partir de la densida C) aplicada en (F1(J), F2 (I))

$\%$ es decir, $\mathrm{Ci}(\mathrm{I}, \mathrm{J})=\mathrm{C}(\mathrm{F} 1(\mathrm{~J}), \mathrm{F} 2(\mathrm{I}))$

$\mathrm{Ci}$

\&calculo de la medida tau

for $J=1: d 1$,

for $I=1: \mathrm{d} 2$

$s 2(I)=F 2(I) * f 2(I)$

$\mathrm{s} 1(\mathrm{~J})=\mathrm{F} 1(\mathrm{~J}) * \mathrm{f} 1(\mathrm{~J})$

end

$\operatorname{s} 12(I, J)=P(I, J) * p(I, J)$

end

$\mathrm{s} 2$;

s1;

s12,

$\mathrm{s} 2=\operatorname{sum}(\mathrm{s} 2(1: \mathrm{d} 2))$

$\mathrm{s} 1=\operatorname{sum}(\mathrm{s} 1(1: \mathrm{d} 1))$

for $I=1: \mathrm{d} 2$,

$\operatorname{si02}(I)=\operatorname{sum}(\operatorname{si2}(I, 1: d 1))$

end

$\mathrm{S} 12=\operatorname{sum}(\mathrm{s} 102(1: \mathrm{d} 2))$

for $J=1: d 1$,

for $I=1: d 2$,

$\operatorname{mf} 2(I, J)=p(I, J) * \operatorname{sum}(p(I, J+1: d 1))$ 


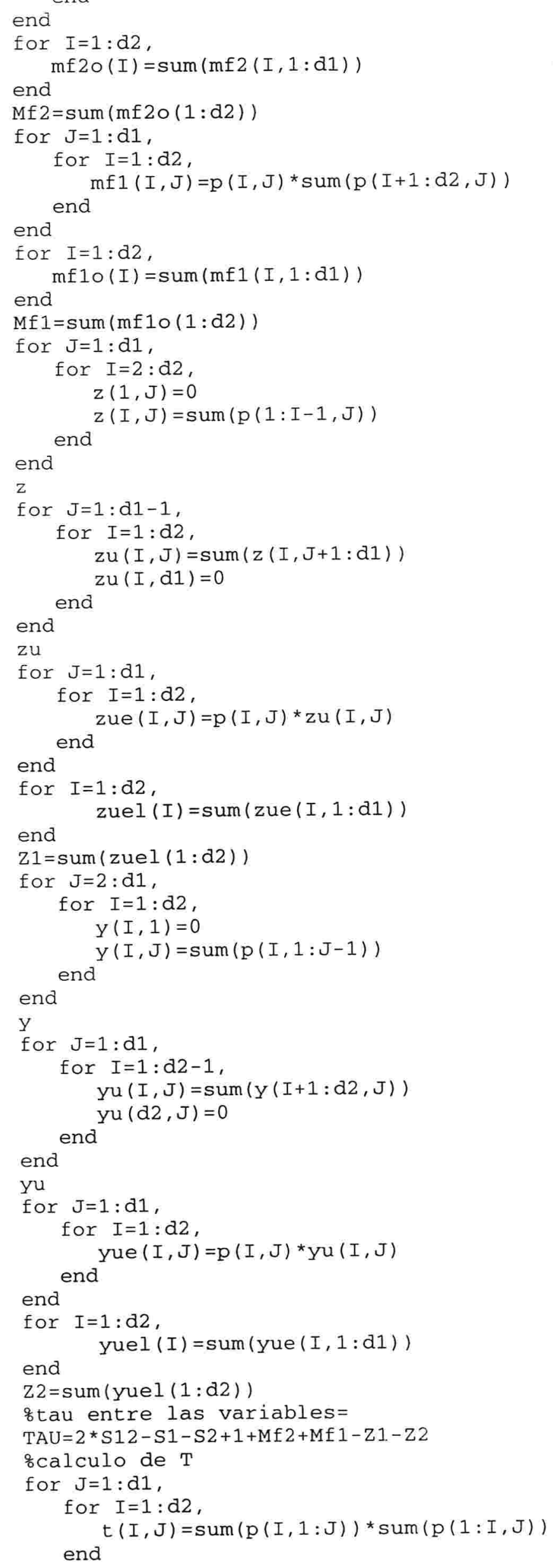




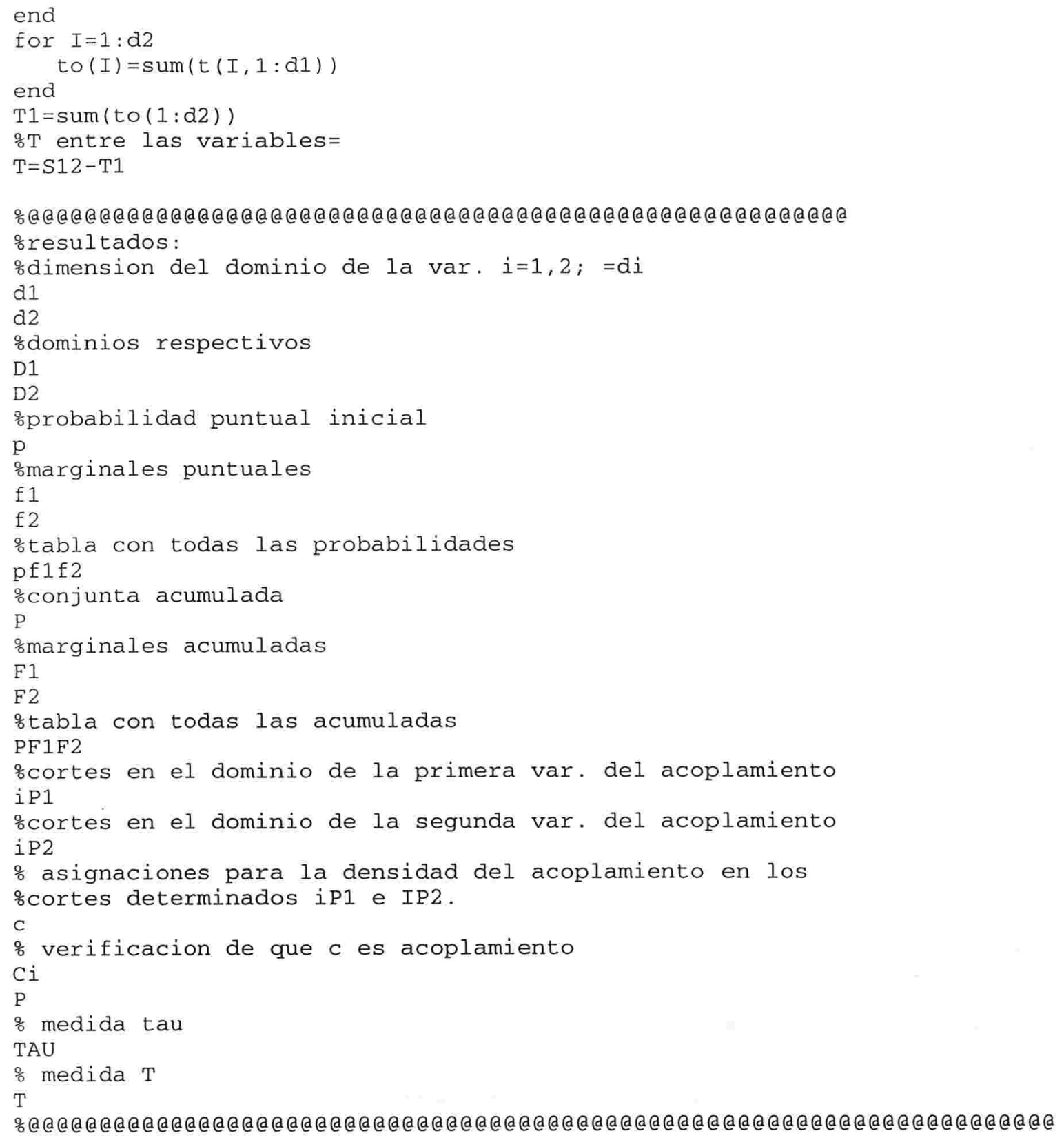




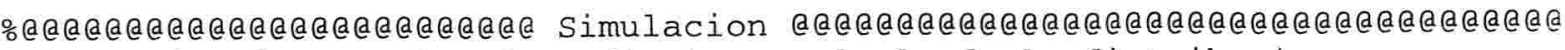
\%simulacion de normales dependientes, calculo de la distribucion

\%acumulada empirica.

\&Descripcion del procedimiento:

$\% 1$ ) generamos $\mathrm{N}$ muestras de 3 normales $(0,1)$, independientes $=x$.

\%2) tranformamos tales normales en, normales dependientes

$\because$ aun con parametros $(0,1)=u$

$\mathrm{N}=500$

$\mathrm{a}=[1 / \operatorname{sqrt}(3) 1 / \operatorname{sqrt}(3) 1 / \operatorname{sqrt}(3) ; 1 / \operatorname{sqrt}(2) 1 / \operatorname{sqrt}(2) 0 ; 100$;

$\%$ matriz de normales independientes, generada aleatoriamente

$\mathrm{x}=\operatorname{randn}(\mathrm{N}, 3)$

$x^{\prime}$

$\mathrm{e}=\mathrm{a} * \mathrm{x}^{\prime}$

$\mathrm{b}=\mathrm{e}^{\prime}$

$u=[b(:, 1)-\operatorname{sqrt}(3), b(:, 2)-\operatorname{sqrt}(2), b(:, 3)] ;$

\%matriz de normales dependientes: la primera columna son los valores :a predecir.Las columnas 2 y 3 corresponden a la base usada para la oprediccion de la primera.

$\mathrm{u}$

\&calculo de la distribucion acumulada empirica, con base en las

ocolumnas 2 y 3 de la matriz u.

$\mathrm{sv}=\mathrm{u}(:, 2)$

[svord, index] =sort (sv)

uex $=u($ index, : )

for $I=1: N$,

$k(I)=0$

for $\mathrm{J}=1: \mathrm{I}$,

if $\operatorname{uex}(J, 3)<=\operatorname{uex}(I, 3)$

$k(I)=k(I)+1$

end

end

end

$\mathrm{aeb}=\mathrm{k}^{\prime} / \mathrm{N}$

vbybt $=[$ uex, aeb $]$

\% matriz completa, descripcion:

$\% 1$ colum=variables a predecir

$\% 2$ y 3 colums= base de prediccion

\% colum=empirica elaborada con base en las dos columnas previas

$\% \quad=$ simiente del predictor

vbybt

\% vamos a ordenar a la matriz vbybt por la norma euclidiana desde

\% las columnas 2 y 3.

for $I=1: N$,

$\operatorname{vbybt}(I, 5)=\operatorname{norm}(\operatorname{vbybt}(I, 2: 3))$

end

tvabubt $(:, 5)$

[ tvord, index] = sort (tv)

FIN=vbybt (index, $1: 5$ )

\% vbybt ordenada por la norma euclideana ( 5 columna $)=F I N$

M1 $=$ mean $(\operatorname{FIN}(:, 1))$

$\mathrm{V} 1=\operatorname{var}(\mathrm{FIN}(:, 1), 1)$

s1 $=$ sqrt $(\mathrm{V} 1)$

plot $\left(1: N, F I N(:, 1), ' s-b ', 1: N, \operatorname{FIN}(:, 4), ' r-{ }^{\prime}\right.$ ')

xlabel('Normas Euclideanas de (U2,U3)')

ylabel ('Valores Verdadeiros=U1. Acum. de (U2,U3)')

legend('Valor Verd.' ', Acum. ', 'corr $=.6955$ ')

title('Acumulada como preditor de monotonicidade')

$\operatorname{corrcoef}([\operatorname{FIN}(:, 1), \operatorname{FIN}(:, 4)])$

\% A continuacion vamos a mostrar como usar el predictor

\& $\mathrm{P}=(\mathrm{M} 1 \wedge 2 / \mathrm{V} 1) * \mathrm{FIN}(:, 4)-\mathrm{M} 1 \wedge 2$.

\% prediciendo u11, de la matriz FIN, supongamos que el valor ull

$\%$ es desconocido, mas los valores u12 y u13 son conocidos. Calcu

\% lamos entonces la media M(1) y la varianza V(1), entre los valores

$\%$ uil, eso fornece informacion pasada de la variabe U1. eses valores

\% calculados, no involucran u11. 
\% el predictor de ul1 sera'dado por;

$\therefore \operatorname{Pred}(1)=(M(1) \wedge 2 / V(1)) *(\operatorname{sum}(\operatorname{FIN}(1: N, 4)) / N)-M(1)^{\wedge} 2$

$\mathrm{TM}=\operatorname{sum}(\operatorname{FIN}(:, 1)) /(\mathrm{N}-1)$

$\operatorname{TV}=(\operatorname{FIN}(:, 1) \cdot * \operatorname{FIN}(:, 1)) /(\mathrm{N}-1)$

$\mathrm{M}=\mathrm{TM}-\mathrm{FIN}(:, 1) /(\mathrm{N}-1)$

$\mathrm{V}=\mathrm{TV}-\mathrm{FIN}(:, 1) \cdot{ }^{*} \mathrm{FIN}(:, 1) /(\mathrm{N}-1)-\mathrm{M}(:) \cdot{ }^{\wedge} 2$

\% predictor para cada punto de la primera columna de FIN

Pred $=\left(M(:) \cdot{ }^{\wedge} 2, / V(:)\right) \cdot{ }^{*} \operatorname{FIN}(:, 4)-M(:) \cdot{ }^{\wedge} 2$

difp=FIN $(:, 1)-\operatorname{Pred}(:)$

esp=mean (difp (:))

$\operatorname{variap}=\operatorname{var}(\operatorname{difp}(:), 1)$

plot $(1: N, F I N(:, 1), ' s-b ', 1: N, \operatorname{Pred}(:), ' r-* ')$

xlabel('Normas Euclideanas de (U2,U3)')

Ylabel ('Valores Verdadeiros=U1. Predictor de U1=Pred')

legend('Valor Verd.' ', 'Pred. ', 'ED=.1072 ', 'VD=.5514')

title('Acumulada corrigida como preditor de U1')

\%fabricando otra simiente de prediccion

for $I=1: N$,

$k c(I)=0$

for $J=1: I$

if $\operatorname{uex}(\mathrm{J}, 3)<=\operatorname{uex}(I, 3)$

if $\operatorname{uex}(\mathrm{J}, 1)<=\operatorname{uex}(I, 1)$

$\operatorname{kc}(I)=\mathrm{kc}(I)+1$

end

end

end

end

acum $=\mathrm{kc}^{\prime} / \mathrm{N}$

omatriz original ordenada por la 2 colum., donde, la ultima colum es

\%la empirica acumulada, de las ternas(U1,U2,U3) .

vbyacum $=[$ uex, aeb]

\% adicionando la norma euclideana de las 2 e 3 columnas, en la 5 colum.

for $I=1: N$,

$\operatorname{vbyacum}(I, 5)=\operatorname{vbybt}(I, 5)$

end

qordenando por la 5 columna

tiv=vbyacum $(:, 5)$

[tivord, index] =sort $($ tiv)

CHE=vbyacum (index, $1: 5$ )

plot $(\operatorname{FIN}(:, 4), \operatorname{CHE}(:, 4)) \%=$ identidad?

plot $\left(1: N, F I N(:, 1), '>-g^{\prime}, 1: N, \operatorname{CHE}(:, 4), \mathrm{m}^{-<}\right.$')

xlabel('Normas Euclideanas de (U2,U3)')

ylabel ('Acum. de (U2, U3). Acum. de (U1, U2, U3)')

legend('Acum. (U2, U3)', 'Acum(U1, U2, U3)')

title('Oscilação entre Acumuladas')

$\%$ predictor con las simientes anteriores

\& dadas por $\operatorname{FIN}(:, 4)$ e por $\operatorname{CHE}(:, 4)$.

for $I=2: N-1$,

if $\operatorname{CHE}(I, 5)-\operatorname{CHE}(I-1,5) \quad c=\operatorname{CHE}(I+1,5)-\operatorname{CHE}(I, 5)$

$\operatorname{Eva}(I)=M(I) \wedge 2 / V(I) * F I N(I, 4)-M(I) / V(I) * C H E(I-1,4)-M(I) \wedge 2-V(I)$ else

$\operatorname{Eva}(I)=M(I) \wedge 2 / V(I) * \operatorname{FIN}(I, 4)-M(I) / V(I) * \operatorname{CHE}(I+1,4)-M(I) \wedge 2-V(I)$

end end

$\operatorname{Eva}(1)=\mathrm{M}(1)^{\wedge} 2 / \mathrm{V}(1) * \mathrm{FIN}(1,4)-\mathrm{M}(1) / \mathrm{V}(1) * \mathrm{CHE}(2,4)-\mathrm{M}(1)^{\wedge} 2-\mathrm{V}(1)$

$\operatorname{Eva}(N)=M(N) \wedge 2 / V(N) \star F I N(N, 4)-M(N) / V(N) * C H E(N-1,4)-M(N) \wedge 2-V(N)$

plot ([FIN $(:, 1), \operatorname{Eva}(:)])$

plot $(1: N, \operatorname{FIN}(:, 1), ' s-b$ ', $1: N, \operatorname{Eva}(:), ' r-* ')$

xlabel('Normas Euclideanas de (U2,U3)')

ylabel ('Valores Verdadeiros=U1. Predictor de U1=Eva')

legend('Valor Verd.' 'Eva', ' $E D=.3317$ ', 'VD=.8509 ')

title('Acumulada corrigida, como preditor de U1')

dife $=\operatorname{FIN}(:, 1)-\operatorname{Eva}(:)$

ese=mean (dife $(:))$

$\operatorname{variae}=\operatorname{var}(\operatorname{dife}(:), 1)$ 


\section{A.2. Condição $T P_{2}\left(R R_{2}\right)$ em pares, Distribuição Conjunta e Operação Bayesiana: Prior/Posterior}

Notação:

$$
\begin{aligned}
\theta & \text { variável a predizer, tomando valores em } R \\
\underline{X}=\left(X_{1}, \cdots, X_{k}\right) & \text { vetor de dados, } \underline{X} \in R^{k}, \text { variáveis base da predição } \\
f_{i} & \text { densidade de prob. de } X_{i}, i=1, \cdots, k \\
f_{\theta, i} & \text { densidade de prob. conjunta de } \theta \text { e } X_{i}, i=1, \cdots, k, \\
f_{\theta, 1, \cdots, k} & \text { densidade de prob. conjunta de } \theta \text { e } \underline{X} \\
f\left(X_{i} \mid \theta\right) & \text { função de verossimilhança } \\
f(\underline{X} \mid \theta) & \text { função de verossimilhança } \\
\xi(\theta) & \text { f.d.p. a priori para } \theta \\
\xi^{*}\left(\theta \mid X_{i}\right) & \text { f.d.p. a posteriori para } \theta \\
\xi^{*}(\theta \mid \underline{X}) & f . d . p . \text { a posteriori para } \theta \\
& \\
& \xi^{*}\left(\theta \mid X_{i}\right)=\frac{f_{\theta, i}\left(\theta, X_{i}\right)}{f_{i}\left(X_{i}\right)}=H\left(X_{i}\right) f\left(X_{i} \mid \theta\right) \xi(\theta),
\end{aligned}
$$

onde

$$
H\left(X_{i}\right):=\left(\int f\left(X_{i} \mid \theta\right) \xi(\theta) d \theta\right)^{-1}
$$

Portanto, se denotamos por $K\left(X_{i}\right):=f_{i}\left(X_{i}\right) H\left(X_{i}\right)$, a conjunta pode ser escrita

$$
f_{\theta, i}\left(\theta, X_{i}\right)=K\left(X_{i}\right) f\left(X_{i} \mid \theta\right) \xi(\theta)
$$

A partir daí é simples deduzir que $f_{\theta, i}\left(\theta, X_{i}\right)$ é $T P_{2}$ se, e somente se, $f\left(X_{i} \mid \theta\right)$ é $T P_{2}$, quando, a última for considerada função de $\theta$ e $X_{i}$.

Finalmente, $\xi^{*}\left(\theta \mid X_{i}\right)$ é $T P_{2}\left(\theta, X_{i}\right) \Leftrightarrow f_{\theta, i}\left(\theta, X_{i}\right)$ é $T P_{2}\left(\theta, X_{i}\right) \Leftrightarrow f\left(X_{i} \mid \theta\right)$ é $T P_{2}\left(\theta, X_{i}\right), i=1, \cdots, k$ 
Continuando com esse raciocínio,

$$
\begin{aligned}
\xi^{*}(\theta \mid \underline{X}) & =\frac{f_{\theta, 1, \cdots, k}(\theta, \underline{X})}{f_{1, \cdots, k}(\underline{X})} \\
& =H(\underline{X}) f(\underline{X} \mid \theta) \xi(\theta) \\
H(\underline{X}) & :=\left(\int f(\underline{X} \mid \theta) \xi(\theta) d \theta\right)^{-1} .
\end{aligned}
$$

pode-se concluir

$\xi^{*}(\theta \mid \underline{X})$ é $T P_{2}\left(\theta, X_{i}\right) \Leftrightarrow f_{\theta, 1, \cdots, k}(\theta, \underline{X})$ é $T P_{2}\left(\theta, X_{i}\right) \Leftrightarrow f(\underline{X} \mid \theta)$ é $T P_{2}\left(\theta, X_{i}\right), 1 \leq i \leq k$.

Conclusões análogas são tiradas para o caso $R R_{2}$ em pares.

Observação : Medida $\tau$ de Kendall e a distribuição a posteriori

Lembremos que, no caso absolutamente contínuo, a medida $\tau_{\theta, X_{i}}=2 T_{\theta, X_{i}}$, onde,

$$
T_{\theta, X_{i}}=\int_{-\infty}^{+\infty} \int_{-\infty}^{+\infty} \int_{-\infty}^{x_{i}^{\prime}} \int_{-\infty}^{\theta^{\prime}}\left[f_{\theta, i}\left(\theta^{\prime}, x_{i}^{\prime}\right) f_{\theta, i}\left(\theta, x_{i}\right)-f_{\theta, i}\left(\theta, x_{i}^{\prime}\right) f_{\theta, i}\left(\theta^{\prime}, x_{i}\right)\right] d \theta d x_{i} d \theta^{\prime} d x_{i}^{\prime}
$$

$i=1, \cdots, k$.

Se definimos $a_{i}\left(x_{i}, x_{i}^{\prime}\right):=f_{i}\left(x_{i}\right) f_{i}\left(x_{i}^{\prime}\right)$, "peso do condicionamento em $X_{i}$ ", $i=$ $1, \cdots, k$.

$T_{\theta, X_{i}}=\int_{-\infty}^{+\infty} \int_{-\infty}^{+\infty} \int_{-\infty}^{x_{i}^{\prime}} \int_{-\infty}^{\theta^{\prime}}\left[\xi^{*}\left(\theta^{\prime} \mid x_{i}^{\prime}\right) \xi^{*}\left(\theta \mid x_{i}\right)-\xi^{*}\left(\theta \mid x_{i}^{\prime}\right) \xi^{*}\left(\theta^{\prime} \mid x_{i}\right)\right] a_{i}\left(x_{i}, x_{i}^{\prime}\right) d \theta d x_{i} d \theta^{\prime} d x_{i}^{\prime}$.

$T_{\theta, X_{i}}$, além de providenciar uma medida de associação na classe das densidades conjuntas $T P_{2}\left(R R_{2}\right)$ de $\theta$ e $X_{i}$, oferece, nesse mesmo sentido, uma medida da condição $T P_{2}\left(R R_{2}\right)$ da posteriori $\theta \mid X_{i}$ com peso dado pelo peso do condicionamento $a_{i}$, antes definido. 


\section{Premissa}

Modificações apresentadas pela distribuição a posteriori para um vetor a predizer, $\underline{\theta}$, quando é alterada a base da predição.

Consideraremos em seguida, a quantidade $\theta$ como um vetor $\underline{\theta}=\left(\theta_{1}, \cdots, \theta_{n}\right)$ tomando valores em $R^{n}$. Lembremos 1.3 .1 e 1.3.3; assim como o Teorema seguinte, que foi o motivador desta seção.

Teorema [Fahmy, S.; C.A.de B.Pereira et al.(1982)]

$\mathrm{Se}$

$$
\begin{gathered}
\xi^{*}(\underline{\theta} \mid \underline{X})=H(\underline{X}) \prod_{i=1}^{k} g_{i}\left(X_{i}, \theta_{i}\right) C(\underline{\theta}) ; \\
g_{i} \quad T P_{2} \mathrm{em} \quad\left(X_{i}, \theta_{i}\right) \forall i=1, \cdots, k ; \\
g_{i} \quad P F_{2}\left(\theta_{i}\right) \quad \text { qdo. } X_{i} \text { é fixo; } \\
C(\underline{\theta}) \quad S-M R R_{2} .
\end{gathered}
$$

Então, Se $\underline{X}=\left(X_{1}, \cdots, X_{k}\right), \underline{X}^{\prime}=\left(X_{1}^{\prime}, \cdots, X_{k}^{\prime}\right), \operatorname{com} X_{i}^{\prime}=X_{i}, X_{j}^{\prime} \geq X_{j}$,

$j=1, \cdots, k j \neq i$

$$
\left.\xi_{i}^{*}\left(\theta_{i} \mid \underline{X}\right)\right)>_{T P_{2}\left(\theta_{i}\right)} \xi_{i}^{*}\left(\theta_{i} \mid \underline{X^{\prime}}\right),
$$

com $i$ fixo, $i \in\{1, \cdots, k\}$. 


\section{Teorema A*}

Se

$$
\xi^{*}(\underline{\theta} \mid \underline{X})=H(\underline{X}) \prod_{i=1}^{k} g_{i}\left(X_{i}, \theta_{i}\right) C(\underline{\theta}),
$$

$g_{i} T P_{2}$ em $\left(X_{i}, \theta_{i}\right), \quad \forall i=1, \cdots, k$.

$$
C(\underline{\theta}) \text { tal que } \forall j \text { e para todo par de vetores } \underline{\theta} \text { e } \underline{\theta}^{\prime}
$$

$\operatorname{com}(\underline{\theta})_{u}=\theta_{u}$ e $\left(\underline{\theta}^{\prime}\right)_{u}=\theta_{u}^{\prime}, u=1, \cdots, k$. verifique

(C) $C(\underline{\theta}) C\left(\underline{\theta}^{\prime}\right) \leq C\left(\theta_{(1)}^{(j-1)} \vee \theta_{(1)}^{(j-1)}, \theta_{j} \wedge \theta_{j}^{\prime}, \theta_{(j+1)}^{(k)} \vee \theta_{j+1}^{\prime(k)}\right)$

$$
C\left(\theta_{(1)}^{(j-1)} \wedge \theta_{1}^{(j-1)}, \theta_{j} \vee \theta_{j}^{\prime}, \theta_{(j+1)}^{(k)} \wedge \theta_{(j+1)}^{(k)}\right)
$$

Então, se $\underline{X}$ e $\underline{X}^{\prime},(\underline{X})_{u}=X_{u},\left(\underline{X}^{\prime}\right)_{u}=X_{u}^{\prime}, u=1, \cdots, k$, tais que

$$
X_{u}^{\prime}=X_{u} \text { para } u \leq j, \quad X_{j}^{\prime}>X_{j} \text { e } X_{u}^{\prime} \leq X_{u} \text { para } u>j ; j \in\{1, \cdots, k\}
$$

$$
\xi_{s, t}^{*}\left(\theta_{s}, \theta_{t} \mid \underline{X}\right)>_{T P_{2}\left(\theta_{s}, \theta_{t}\right)} \xi_{s, t}^{*}\left(\theta_{s}, \theta_{t} \mid \underline{X^{\prime}}\right) \quad t \neq s, \quad t, s<j
$$

$(A .2) \xi_{1, \cdots, j-1}^{*}\left(\theta_{1}, \cdots, \theta_{j-1} \mid \underline{X}\right)>_{T P_{2}\left(\theta_{1}, \cdots, \theta_{j-1}\right)} \xi_{1, \cdots, j-1}^{*}\left(\theta_{1}, \cdots, \theta_{j-1} \mid \underline{X}^{\prime}\right), \quad j \in\{2, \cdots, k\} ;$

$\left.(A .3) \quad \xi_{1, \cdots, j-1, j+1, \cdots, k}^{*}\left(\theta_{(1)}^{(j-1)}, \theta_{(j+1)}^{(k)}\right) \mid \underline{X}\right)$

$$
>_{T P_{2}\left(\theta_{(1)}^{(j-1)}, \theta_{(j+1)}^{(k)}\right)} \xi_{1, \cdots, j-1, j+1, \cdots, k}^{*}\left(\theta_{(1)}^{(j-1)}, \theta_{(j+1)}^{(k)} \mid \underline{X}^{\prime}\right)
$$

$j \in\{2, \cdots, k-1\}$. 
Prova: (A.1) basta demonstrá-lo para $t=1, s=2, j=3$,

$$
\text { - } \underline{X}=\left(X_{1}, X_{2}, X_{3}, X_{(4)}^{(k)}\right), \underline{X^{\prime}}=\left(X_{1}, X_{2}, X_{3}^{\prime}, X_{(4)}^{(k)}\right)
$$

oSe $k>3$ temos

$\xi_{1,2}^{*}\left(\theta_{1}, \theta_{2} \mid \underline{X}\right)$

$$
=H(\underline{X}) g_{1}\left(X_{1}, \theta_{1}\right) g_{2}\left(X_{2}, \theta_{2}\right) \int g_{3}\left(X_{3}, \theta_{3}\right)\left[\int \cdots \int C(\underline{\theta}) \prod_{i=4}^{k} g_{i}\left(X_{i}, \theta_{i}\right) d \theta_{i}\right] d \theta_{3}
$$

Consideremos agora dois pares de pontos $\left(\theta_{1}, \theta_{2}\right)$ e $\left(\theta_{1}^{\prime}, \theta_{2}^{\prime}\right)$, arbitrários

$$
\text { (1) } \xi_{1,2}^{*}(\cdot, \cdot \mid \underline{X})>_{T P_{2}(\cdot, \cdot)} \xi_{1,2}^{*}\left(\cdot, \cdot \mid \underline{X^{\prime}}\right)
$$

se, e somente se,

(2) $\xi_{1,2}^{*}\left(\theta_{1} \vee \theta_{1}^{\prime}, \theta_{2} \vee \theta_{2}^{\prime} \mid \underline{X}\right) \xi_{1,2}^{*}\left(\theta_{1} \wedge \theta_{1}^{\prime}, \theta_{2} \wedge \theta_{2}^{\prime} \mid \underline{X}^{\prime}\right) \geq \xi_{1,2}^{*}\left(\theta_{1}^{\prime}, \theta_{2}^{\prime} \mid \underline{X}\right) \xi_{1,2}^{*}\left(\theta_{1}, \theta_{2} \mid \underline{X^{\prime}}\right)$

se, e somente se,

$$
\begin{aligned}
& g_{1}\left(x_{1}, \theta_{1} \vee \theta_{1}^{\prime}\right) g_{2}\left(x_{2}, \theta_{2} \vee \theta_{2}^{\prime}\right) \int g_{3}\left(x_{3}, \theta_{3}\right)\left[\int \cdots \int C\left(\theta_{1} \vee \theta_{1}^{\prime}, \theta_{2} \vee \theta_{2}^{\prime}, \theta_{(3)}^{(k)}\right)\right. \\
& \left.\prod_{i=4}^{k} g_{i}\left(x_{i}, \theta_{i}\right) d \theta_{i}\right] d \theta_{3} g_{1}\left(x_{1}, \theta_{1} \wedge \theta_{1}^{\prime}\right) g_{2}\left(x_{2}, \theta_{2} \wedge \theta_{2}^{\prime}\right) \int g_{3}\left(x_{3}^{\prime}, \theta_{3}\right) \\
& {\left[\int \cdots \int C\left(\theta_{1} \wedge \theta_{1}^{\prime}, \theta_{2} \wedge \theta_{2}^{\prime}, \theta_{(3)}^{(k)}\right) \prod_{i=4}^{k} g_{i}\left(x_{i}, \theta_{i}\right) d \theta_{i}\right] d \theta_{3}} \\
& \geq g_{1}\left(x_{1}, \theta_{1}^{\prime}\right) g_{2}\left(x_{2}, \theta_{2}^{\prime}\right) \int g_{3}\left(x_{3}, \theta_{3}\right)\left[\int \cdots \int C\left(\theta_{1}^{\prime}, \theta_{2}^{\prime}, \theta_{(3)}^{(k)}\right) \prod_{i=4}^{k} g_{i}\left(x_{i}, \theta_{i}\right) d \theta_{i}\right] d \theta_{3} \\
& g_{1}\left(x_{1}, \theta_{1}\right) g_{2}\left(x_{2}, \theta_{2}\right) \int g_{3}\left(x_{3}^{\prime}, \theta_{3}\right)\left[\int \cdots \int C\left(\theta_{1}, \theta_{2}, \theta_{(3)}^{(k)}\right) \prod_{i=4}^{k} g_{i}\left(x_{i}, \theta_{i}\right) d \theta_{i}\right] d \theta_{3}
\end{aligned}
$$

mas, como $g_{i}$ é $T P_{2}$ em $\left(X_{i}, \theta_{i}\right) \forall i=1, \cdots, k$, a desigualdade anterior é equivalente a 
(3) $\int g_{3}\left(x_{3}, \theta_{3}\right)\left[\int \cdots \int C\left(\theta_{1} \vee \theta_{1}^{\prime}, \theta_{2} \vee \theta_{2}^{\prime}, \theta_{(3)}^{(k)}\right) \prod_{i=4}^{k} g_{i}\left(x_{i}, \theta_{i}\right) d \theta_{i}\right] d \theta_{3}$

$\int g_{3}\left(x_{3}^{\prime}, \theta_{3}\right)\left[\int \cdots \int C\left(\theta_{1} \wedge \theta_{1}^{\prime}, \theta_{2} \wedge \theta_{2}^{\prime}, \theta_{(3)}^{(k)}\right) \prod_{i=4}^{k} g_{i}\left(x_{i}, \theta_{i}\right) d \theta_{i}\right] d \theta_{3}$

$\geq \int g_{3}\left(x_{3}, \theta_{3}\right)\left[\int \cdots \int C\left(\theta_{1}^{\prime}, \theta_{2}^{\prime}, \theta_{(3)}^{(k)}\right) \prod_{i=4}^{k} g_{i}\left(x_{i}, \theta_{i}\right) d \theta_{i}\right] d \theta_{3}$

$\int g_{3}\left(x_{3}^{\prime}, \theta_{3}\right)\left[\int \cdots \int C\left(\theta_{1}, \theta_{2}, \theta_{(3)}^{(k)}\right) \prod_{i=4}^{k} g_{i}\left(x_{i}, \theta_{i}\right) d \theta_{i}\right] d \theta_{3}$

Denotemos por

$$
G\left(\theta_{1}, \theta_{2}, \theta_{3}, x_{(4)}^{(k)}\right):=\int \cdots \int C\left(\theta_{1}, \theta_{2}, \theta_{(3)}^{(k)}\right) \prod_{i=4}^{k} g_{i}\left(x_{i}, \theta_{i}\right) d \theta_{i}
$$

Afirmação:

(4) $G\left(\theta_{1} \vee \theta_{1}^{\prime}, \theta_{2} \vee \theta_{2}^{\prime}, \theta_{3} \wedge \theta_{3}^{\prime}, x_{(4)}^{(k)}\right) G\left(\theta_{1} \wedge \theta_{1}^{\prime}, \theta_{2} \wedge \theta_{2}^{\prime}, \theta_{3} \vee \theta_{3}^{\prime}, x_{(4)}^{(k)}\right)$

$$
\geq G\left(\theta_{1}, \theta_{2}, \theta_{3}, x_{(4)}^{(k)}\right) G\left(\theta_{1}^{\prime}, \theta_{2}^{\prime}, \theta_{3}^{\prime}, x_{(4)}^{(k)}\right)
$$

Prova, definindo

$$
\begin{aligned}
& f_{1}\left(\theta_{(4)}^{(k)}\right):=C\left(\theta_{1}, \theta_{2}, \theta_{3}, \theta_{(4)}^{(k)}\right) \prod_{i=4}^{k} g_{i}\left(x_{i}, \theta_{i}\right) \\
& f_{2}\left(\theta_{(4)}^{(k)}\right):=C\left(\theta_{1}^{\prime}, \theta_{2}^{\prime}, \theta_{3}^{\prime}, \theta_{(4)}^{(k)}\right) \prod_{i=4}^{k} g_{i}\left(x_{i}, \theta_{i}\right) \\
& f_{3}\left(\theta_{(4)}^{(k)}\right):=C\left(\theta_{1} \wedge \theta_{1}^{\prime}, \theta_{2} \wedge \theta_{2}^{\prime}, \theta_{3} \vee \theta_{3}^{\prime}, \theta_{(4)}^{(k)}\right) \prod_{i=4}^{k} g_{i}\left(x_{i}, \theta_{i}\right) \\
& f_{4}\left(\theta_{(4)}^{(k)}\right):=C\left(\theta_{1} \vee \theta_{1}^{\prime}, \theta_{2} \vee \theta_{2}^{\prime}, \theta_{3} \wedge \theta_{3}^{\prime}, \theta_{(4)}^{(k)}\right) \prod_{i=4}^{k} g_{i}\left(x_{i}, \theta_{i}\right)
\end{aligned}
$$


é possível verificar que para

$$
\begin{gathered}
\underline{v}=\left(\theta_{4}, \theta_{5}, \cdots, \theta_{k}\right) \text { e } \underline{w}=\left(\theta_{4}^{\prime}, \theta_{5}^{\prime}, \cdots, \theta_{k}^{\prime}\right), \\
f_{1}(\underline{v}) f_{2}(\underline{w}) \leq f_{3}(\underline{v} \wedge \underline{w}) f_{4}(\underline{v} \vee \underline{w}),
\end{gathered}
$$

ou, equivalentemente (pois as $g_{i}$ são $T P_{2}$ ),

$C(\underline{\theta}) C\left(\underline{\theta}^{\prime}\right) \leq C\left(\theta_{(1)}^{(j-1)} \vee \theta_{(1)}^{\prime(j-1)}, \theta_{j} \wedge \theta_{j}^{\prime}, \theta_{(j+1)}^{(k)} \vee \theta_{j+1}^{\prime(k)}\right)$

$$
C\left(\theta_{(1)}^{(j-1)} \wedge \theta_{1}^{(j-1)}, \theta_{j} \vee \theta_{j}^{\prime}, \theta_{(j+1)}^{(k)} \wedge \theta_{(j+1)}^{(k)}\right)
$$

Esta última desigualdade vale por hipótese. Agora, por 1.3.1, temos:

$$
\int f_{1}\left(\theta_{(4)}^{(k)}\right) d \theta_{(4)}^{(k)} \int f_{2}\left(\theta_{(4)}^{(k)}\right) d \theta_{(4)}^{(k)} \leq \int f_{3}\left(\theta_{(4)}^{(k)}\right) d \theta_{(4)}^{(k)} \int f_{4}\left(\theta_{(4)}^{(k)}\right) d \theta_{(4)}^{(k)}
$$

e, assim, provamos (4). (3) é equivalente a

(5)

$$
\begin{aligned}
& \int g_{3}\left(x_{3}, \theta_{3}\right) G\left(\theta_{1} \vee \theta_{1}^{\prime}, \theta_{2} \vee \theta_{2}^{\prime}, \theta_{3}, x_{(4)}^{(k)}\right) d \theta_{3} \int g_{3}\left(x_{3}^{\prime}, \theta_{3}\right) G\left(\theta_{1} \wedge \theta_{1}^{\prime}, \theta_{2} \wedge \theta_{2}^{\prime}, \theta_{3}, x_{(4)}^{(k)}\right) d \theta_{3} \\
& \geq \int g_{3}\left(x_{3}, \theta_{3}\right) G\left(\theta_{1}^{\prime}, \theta_{2}^{\prime}, \theta_{3}, x_{(4)}^{(k)}\right) d \theta_{3} \int g_{3}\left(x_{3}^{\prime}, \theta_{3}\right) G\left(\theta_{1}, \theta_{2}, \theta_{3}, x_{(4)}^{(k)}\right) d \theta_{3}
\end{aligned}
$$

Para a prova de (5), definamos

$$
\begin{aligned}
f_{1}^{*}\left(\theta_{3}\right) & :=g_{3}\left(x_{3}, \theta_{3}\right) G\left(\theta_{1}^{\prime}, \theta_{2}^{\prime}, \theta_{3}, x_{(4)}^{(k)}\right) \\
f_{2}^{*}\left(\theta_{3}\right) & :=g_{3}\left(x_{3}^{\prime}, \theta_{3}\right) G\left(\theta_{1}, \theta_{2}, \theta_{3}, x_{(4)}^{(k)}\right) \\
f_{3}^{*}\left(\theta_{3}\right) & :=g_{3}\left(x_{3}, \theta_{3}\right) G\left(\theta_{1} \vee \theta_{1}^{\prime}, \theta_{2} \vee \theta_{2}^{\prime}, \theta_{3}, x_{(4)}^{(k)}\right) \\
f_{4}^{*}\left(\theta_{3}\right) & :=g_{3}\left(x_{3}^{\prime}, \theta_{3}\right) G\left(\theta_{1} \wedge \theta_{1}^{\prime}, \theta_{2} \wedge \theta_{2}^{\prime}, \theta_{3}, x_{(4)}^{(k)}\right),
\end{aligned}
$$

verificando que

$$
f_{1}^{*}\left(\theta_{3}^{\prime}\right) f_{2}^{*}\left(\theta_{3}\right) \leq f_{3}^{*}\left(\theta_{3} \wedge \theta_{3}^{\prime}\right) f_{4}^{*}\left(\theta_{3} \vee \theta_{3}^{\prime}\right)
$$


por ser $g_{3}, T P_{2}$; então, por 1.3.1, provamos (5), equivalentemente (1).

oSe $k=3$

$$
\xi_{1,2}^{*}\left(\theta_{1}, \theta_{2} \mid \underline{X}\right)=H(\underline{X}) g_{1}\left(X_{1}, \theta_{1}\right) g_{2}\left(X_{2}, \theta_{2}\right) \int g_{3}\left(X_{3}, \theta_{3}\right) C\left(\theta_{1}, \theta_{2}, \theta_{3}\right) d \theta_{3}
$$

Logo, como $g_{1}$ e $g_{2}$ são $T P_{2}$, (1) é verificada através da seguinte

(6) $g_{3}\left(x_{3}, \theta_{3}\right) C\left(\theta_{1} \vee \theta_{1}^{\prime}, \theta_{2} \vee \theta_{2}^{\prime}, \theta_{3}\right) g_{3}\left(x_{3}^{\prime}, \theta_{3}\right) C\left(\theta_{1} \wedge \theta_{1}^{\prime}, \theta_{2} \wedge \theta_{2}^{\prime}, \theta_{3}\right)$

$$
\geq g_{3}\left(x_{3}^{\prime}, \theta_{3}\right) C\left(\theta_{1}, \theta_{2}, \theta_{3}\right) g_{3}\left(x_{3}, \theta_{3}\right) C\left(\theta_{1}^{\prime}, \theta_{2}^{\prime}, \theta_{3}\right) \text {. }
$$

Esta última desigualdade é verificada a partir das hipóteses sobre $C, g_{3}$ e o uso de 1.3.1.

$$
\begin{gathered}
\cdot \underline{X}=\left(X_{1}, X_{2}, X_{3}, X_{4}, \cdots, X_{k}\right), \underline{X^{\prime}}=\left(X_{1}, X_{2}, X_{3}^{\prime}, X_{4}^{\prime}, \cdots, X_{k}\right) \\
\operatorname{com} X_{3}^{\prime}>X_{3}, X_{4}^{\prime}<X_{4}
\end{gathered}
$$

Neste caso (3) fica substituída por

(a3)

$$
\begin{aligned}
& \int g_{3}\left(x_{3}, \theta_{3}\right)\left[\int g_{4}\left(x_{4}, \theta_{4}\right)\left(\int \cdots \int C\left(\theta_{1} \vee \theta_{1}^{\prime}, \theta_{2} \vee \theta_{2}^{\prime}, \theta_{(3)}^{(k)}\right) \prod_{i=5}^{k} g_{i}\left(x_{i}, \theta_{i}\right) d \theta_{i}\right) d \theta_{4}\right] d \theta_{3} \\
& \int g_{3}\left(x_{3}^{\prime}, \theta_{3}\right)\left[\int g_{4}\left(x_{4}^{\prime}, \theta_{4}\right)\left(\int \cdots \int C\left(\theta_{1} \wedge \theta_{1}^{\prime}, \theta_{2} \wedge \theta_{2}^{\prime}, \theta_{(3)}^{(k)}\right) \prod_{i=5}^{k} g_{i}\left(x_{i}, \theta_{i}\right) d \theta_{i}\right) d \theta_{4}\right] d \theta_{3} \\
& \geq \int g_{3}\left(x_{3}, \theta_{3}\right)\left[\int g_{4}\left(x_{4}, \theta_{4}\right)\left(\int \cdots \int C\left(\theta_{1}^{\prime}, \theta_{2}^{\prime}, \theta_{(3)}^{(k)}\right) \prod_{i=5}^{k} g_{i}\left(x_{i}, \theta_{i}\right) d \theta_{i}\right) d \theta_{4}\right] d \theta_{3} \\
& \int g_{3}\left(x_{3}^{\prime}, \theta_{3}\right)\left[\int g_{4}\left(x_{4}^{\prime}, \theta_{4}\right)\left(\int \cdots \int C\left(\theta_{1}, \theta_{2}, \theta_{(3)}^{(k)}\right) \prod_{i=5}^{k} g_{i}\left(x_{i}, \theta_{i}\right) d \theta_{i}\right) d \theta_{4}\right] d \theta_{3}
\end{aligned}
$$

Fixando $\left(x_{5}, \cdots, x_{k}\right)$, definamos

$$
H\left(\theta_{1}, \theta_{2}, \theta_{3}, x_{4}\right):=\int g_{4}\left(x_{4}, \theta_{4}\right)\left(\int \cdots \int C\left(\theta_{1}, \theta_{2}, \theta_{3}, \cdots, \theta_{k}\right) \prod_{i=5}^{k} g_{i}\left(x_{i}, \theta_{i}\right) d \theta_{i}\right) d \theta_{4}
$$


Deste jeito fica (a3) substituída por

$$
\begin{aligned}
& \int g_{3}\left(x_{3}, \theta_{3}\right) H\left(\theta_{1} \vee \theta_{1}^{\prime}, \theta_{2} \vee \theta_{2}^{\prime}, \theta_{3}, x_{4}\right) d \theta_{3} \int g_{3}\left(x_{3}^{\prime}, \theta_{3}\right) H\left(\theta_{1} \wedge \theta_{1}^{\prime}, \theta_{2} \wedge \theta_{2}^{\prime}, \theta_{3}, x_{4}^{\prime}\right) d \theta_{3} \\
& \geq \int g_{3}\left(x_{3}, \theta_{3}\right) H\left(\theta_{1}^{\prime}, \theta_{2}^{\prime}, \theta_{3}, x_{4}\right) d \theta_{3} \int g_{3}\left(x_{3}^{\prime}, \theta_{3}\right) H\left(\theta_{1}, \theta_{2}, \theta_{3}, x_{4}^{\prime}\right) d \theta_{3} .
\end{aligned}
$$

Afirmação: para $\theta_{3}^{\prime}$ e $\theta_{3}$ ambos arbitrários,

$$
\begin{aligned}
& g_{3}\left(x_{3}, \theta_{3} \wedge \theta_{3}^{\prime}\right) H\left(\theta_{1} \vee \theta_{1}^{\prime}, \theta_{2} \vee \theta_{2}^{\prime}, \theta_{3} \wedge \theta_{3}^{\prime}, x_{4}\right) \\
& g_{3}\left(x_{3}^{\prime}, \theta_{3} \vee \theta_{3}^{\prime}\right) H\left(\theta_{1} \wedge \theta_{1}^{\prime}, \theta_{2} \wedge \theta_{2}^{\prime}, \theta_{3} \vee \theta_{3}^{\prime}, x_{4}^{\prime}\right) \\
& \geq g_{3}\left(x_{3}, \theta_{3}^{\prime}\right) H\left(\theta_{1}^{\prime}, \theta_{2}^{\prime}, \theta_{3}^{\prime}, x_{4}\right) g_{3}\left(x_{3}^{\prime}, \theta_{3}\right) H\left(\theta_{1}, \theta_{2}, \theta_{3}, x_{4}^{\prime}\right)
\end{aligned}
$$

Deste modo, podemos aplicar 1.3.1- concluindo com a prova da validade de (a3).

Prova-se a última afirmação usando 1.3.1, e as condições $x_{4}>x_{4}^{\prime}, g_{4} T P_{2}$.

Prova de ( A.2)

(7) $\xi_{1, \cdots, j-1}^{*}\left(\theta_{1}, \theta_{2}, \cdots, \theta_{j-1} \mid \underline{X}\right)$

$$
=H(\underline{X}) g_{1}\left(X_{1}, \theta_{1}\right) \cdots g_{j-1}\left(X_{j-1}, \theta_{j-1}\right) \int \cdots \int C(\underline{\theta}) \prod_{i=j}^{k} g_{i}\left(X_{i}, \theta_{i}\right) d \theta_{i}
$$

Consideremos agora dois pares de pontos $\left(\theta_{1}, \cdots, \theta_{j-1}\right)$ e $\left(\theta_{1}^{\prime}, \cdots, \theta_{j-1}^{\prime}\right)$ arbitrários. Supondo

$$
\underline{X}=\left(X_{1}, \cdots, X_{j-1}, X_{j}, X_{j+1}, \cdots, X_{k}\right) \text { e } \underline{X^{\prime}}=\left(X_{1}, \cdots, X_{j-1}, X_{j}^{\prime}, X_{j+1}^{\prime}, \cdots, X_{k}^{\prime}\right)
$$

$\operatorname{com} X_{j}^{\prime}>X_{j} X_{s}^{\prime} \leq X_{s} s>j$ notemos que (A.2) é equivalente a 
(8)

$$
\begin{aligned}
& g_{1}\left(x_{1}, \theta_{1} \vee \theta_{1}^{\prime}\right) \cdots g_{j-1}\left(x_{j-1}, \theta_{j-1} \vee \theta_{j-1}^{\prime}\right)\left[\int \cdots \int C\left(\theta_{1} \vee \theta_{1}^{\prime}, \cdots, \theta_{j-1} \vee \theta_{j-1}^{\prime}, \theta_{(j)}^{(k)}\right)\right. \\
& \left.\prod_{i=j}^{k} g_{i}\left(x_{i}, \theta_{i}\right) d \theta_{i}\right] g_{1}\left(x_{1}, \theta_{1} \wedge \theta_{1}^{\prime}\right) \cdots g_{j-1}\left(x_{j-1}, \theta_{j-1} \wedge \theta_{j-1}^{\prime}\right) \\
& {\left[\int \cdots \int C\left(\theta_{1} \wedge \theta_{1}^{\prime}, \cdots, \theta_{j-1} \wedge \theta_{j-1}^{\prime}, \theta_{(j)}^{(k)}\right) \prod_{i=j}^{k} g_{i}\left(x_{i}^{\prime}, \theta_{i}\right) d \theta_{i}\right]} \\
& \geq g_{1}\left(x_{1}, \theta_{1}^{\prime}\right) \cdots g_{j-1}\left(x_{j-1}, \theta_{j-1}^{\prime}\right)\left[\int \cdots \int C\left(\theta_{1}^{\prime}, \cdots, \theta_{j-1}^{\prime}, \theta_{(j)}^{(k)}\right) \prod_{i=j}^{k} g_{i}\left(x_{i}, \theta_{i}\right) d \theta_{i}\right] \\
& g_{1}\left(x_{1}, \theta_{1}\right) \cdots g_{j-1}\left(x_{j-1}, \theta_{j-1}\right)\left[\int \cdots \int C\left(\theta_{1}, \cdots, \theta_{j-1}, \theta_{(j)}^{(k)}\right) \prod_{i=j}^{k} g_{i}\left(x_{i}^{\prime}, \theta_{i}\right) d \theta_{i}\right]
\end{aligned}
$$

mas, como $g_{i}$ é $T P_{2}$ em $\left(X_{i}, \theta_{i}\right) \forall i=1, \cdots, k,(8)$ é equivalente a

$$
\begin{aligned}
& \text { (9) } \quad \int \cdots \int C\left(\theta_{1} \vee \theta_{1}^{\prime}, \cdots, \theta_{j-1} \vee \theta_{j-1}^{\prime}, \theta_{(j)}^{(k)}\right) \prod_{i=j}^{k} g_{i}\left(x_{i}, \theta_{i}\right) d \theta_{i} \\
& \int \cdots \int C\left(\theta_{1} \wedge \theta_{1}^{\prime}, \cdots, \theta_{j-1} \wedge \theta_{j-1}^{\prime}, \theta_{(j)}^{(k)}\right) \prod_{i=j}^{k} g_{i}\left(x_{i}^{\prime}, \theta_{i}\right) d \theta_{i} \\
& \geq \int \cdots \int C\left(\theta_{1}^{\prime}, \cdots, \theta_{j-1}^{\prime}, \theta_{(j)}^{(k)}\right) \prod_{i=j}^{k} g_{i}\left(x_{i}, \theta_{i}\right) d \theta_{i} \\
& \int \cdots \int C\left(\theta_{1}, \cdots, \theta_{j-1}, \theta_{(j)}^{(k)}\right) \prod_{i=j}^{k} g_{i}\left(x_{i}^{\prime}, \theta_{i}\right) d \theta_{i} .
\end{aligned}
$$

o Consideremos o caso em que $X_{s}^{\prime}=X_{s} \forall s>j$, denotando por

$$
G\left(\theta_{1}, \cdots, \theta_{j-1}, \theta_{j}, x_{(j+1)}^{(k)}\right):=\int \cdots \int C\left(\theta_{1}, \cdots, \theta_{j-1}, \theta_{(j)}^{(k)}\right) \prod_{i=j+1}^{k} g_{i}\left(x_{i}, \theta_{i}\right) d \theta_{i} .
$$




\section{Afirmação}

$$
\begin{gathered}
G\left(\theta_{1} \vee \theta_{1}^{\prime}, \cdots, \theta_{j-1} \vee \theta_{j-1}^{\prime}, \theta_{j} \wedge \theta_{j}^{\prime}, x_{(j+1)}^{(k)}\right) G\left(\theta_{1} \wedge \theta_{1}^{\prime}, \cdots, \theta_{j-1} \wedge \theta_{j-1}^{\prime}, \theta_{j} \vee \theta_{j}^{\prime}, x_{(j+1)}^{(k)}\right) \\
\geq G\left(\theta_{1}, \cdots, \theta_{j-1}, \theta_{j}, x_{(j+1)}^{(k)}\right) G\left(\theta_{1}^{\prime}, \cdots, \theta_{j-1}^{\prime}, \theta_{j}^{\prime}, x_{(j+1)}^{(k)}\right)
\end{gathered}
$$

só adaptando a prova feita para (4). Logo (9) fica

$$
\text { (11) } \begin{aligned}
& \int g_{j}\left(x_{j}, \theta_{j}\right) G\left(\theta_{1} \vee \theta_{1}^{\prime}, \cdots, \theta_{j-1} \vee \theta_{j-1}^{\prime}, \theta_{j}, x_{(j+1)}^{(k)}\right) d \theta_{j} \\
& \int g_{j}\left(x_{j}^{\prime}, \theta_{j}\right) G\left(\theta_{1} \wedge \theta_{1}^{\prime}, \cdots, \theta_{j-1} \wedge \theta_{j-1}^{\prime}, \theta_{j}, x_{(j+1)}^{(k)}\right) d \theta_{j} \\
\geq & \int g_{j}\left(x_{j}, \theta_{j}\right) G\left(\theta_{1}^{\prime}, \cdots, \theta_{j-1}^{\prime}, \theta_{j}, x_{(j+1)}^{(k)}\right) d \theta_{j} \\
& \int g_{j}\left(x_{j}^{\prime}, \theta_{j}\right) G\left(\theta_{1}, \cdots, \theta_{j-1}, \theta_{j}, x_{(j+1)}^{(k)}\right) d \theta_{j},
\end{aligned}
$$

e, com prova análoga à feita para (5), usando (10), fica provada (11). Logo, vale (A.2).

○ O caso geral $\left(X_{s}^{\prime} \leq X_{s} \forall s>j\right)$ é tratado como na prova de (A.1).

Prova de (A.3)

(12) $\xi_{1, \cdots, j-1, j+1, \cdots, k}^{*}\left(\theta_{(1)}^{(j-1)}, \theta_{(j+1)}^{(k)} \mid \underline{X}\right)$

$$
=H(\underline{X}) \prod_{\substack{i=1 \\ i \neq j}}^{k} g_{i}\left(X_{i}, \theta_{i}\right) \int g_{j}\left(X_{j}, \theta_{j}\right) C(\underline{\theta}) d \theta_{j}
$$

Consideremos agora dois pares de pontos arbitrários

$$
\left(\theta_{1}, \cdots, \theta_{j-1}, \theta_{j+1}, \cdots, \theta_{k}\right),\left(\theta_{1}^{\prime}, \cdots, \theta_{j-1}^{\prime}, \theta_{j+1}^{\prime}, \cdots, \theta_{k}^{\prime}\right)
$$


Supondo

$\underline{X}=\left(X_{1}, \cdots, X_{j-1}, X_{j}, X_{j+1}, \cdots, X_{k}\right)$ e $\underline{X^{\prime}}=\left(X_{1}, \cdots, X_{j-1}, X_{j}^{\prime}, X_{j+1}^{\prime}, \cdots, X_{k}^{\prime}\right)$

$\operatorname{com} X_{j}^{\prime}>X_{j} X_{s}^{\prime} \leq X_{s} s>j$

o Caso em que $X_{s}^{\prime}=X_{s} s>j:$ notemos que (A.3) é equivalente a,

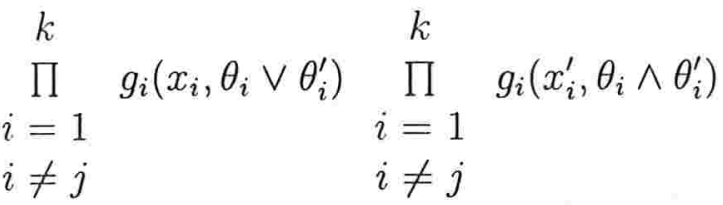

$$
\begin{aligned}
& \int g_{j}\left(x_{j}, \theta_{j}\right) C\left(\theta_{(1)}^{(j-1)} \vee \theta_{(1)}^{(j-1)}, \theta_{j}, \theta_{(j+1)}^{(k)} \vee \theta_{(j+1)}^{(k)}\right) d \theta_{j} \\
& \int g_{j}\left(x_{j}^{\prime}, \theta_{j}\right) C\left(\theta_{(1)}^{(j-1)} \wedge \theta_{(1)}^{(j-1)}, \theta_{j}, \theta_{(j+1)}^{(k)} \wedge \theta_{(j+1)}^{(k)}\right) d \theta_{j} \\
& \geq \prod_{\substack{i=1 \\
i \neq j}}^{k} g_{i}\left(x_{i}, \theta_{i}^{\prime}\right) \int g_{j}\left(x_{j}, \theta_{j}\right) C\left(\theta_{(1)}^{(j-1)}, \theta_{j}, \theta_{(j+1)}^{(k)}\right) d \theta_{j} \\
& \prod_{\substack{i=1 \\
i \neq j}}^{k} g_{i}\left(x_{i}^{\prime}, \theta_{i}\right) \int g_{j}\left(x_{j}^{\prime}, \theta_{j}\right) C\left(\theta_{(1)}^{(k)}\right) d \theta_{j},
\end{aligned}
$$

mas como $X_{s}^{\prime}=X_{s}, s>j$ e $g_{i}$ é $T P_{2}$ a desigualdade anterior é equivalente a

$$
\begin{aligned}
& \int g_{j}\left(x_{j}, \theta_{j}\right) C\left(\theta_{(1)}^{(j-1)} \vee \theta_{(1)}^{(j-1)}, \theta_{j}, \theta_{(j+1)}^{(k)} \vee \theta_{(j+1)}^{(k)}\right) d \theta_{j} \\
& \int g_{j}\left(x_{j}^{\prime}, \theta_{j}\right) C\left(\theta_{(1)}^{(j-1)} \wedge \theta_{(1)}^{(j-1)}, \theta_{j}, \theta_{(j+1)}^{(k)} \wedge \theta_{(j+1)}^{(k)}\right) d \theta_{j} \\
& \geq \int g_{j}\left(x_{j}, \theta_{j}\right) C\left(\theta_{(1)}^{(j-1)}, \theta_{j}, \theta_{(j+1)}^{(k)}\right) d \theta_{j} \int g_{j}\left(x_{j}^{\prime}, \theta_{j}\right) C\left(\theta_{(1)}^{(k)}\right) d \theta_{j},
\end{aligned}
$$


se,

$$
\begin{aligned}
f_{1}\left(\theta_{j}\right) & :=C\left(\theta_{(1)}^{\prime(j-1)}, \theta_{j}, \theta_{(j+1)}^{\prime(k)}\right) g_{j}\left(x_{j}, \theta_{j}\right) \\
f_{2}\left(\theta_{j}\right) & :=C\left(\theta_{(1)}^{(k)}\right) g_{j}\left(x_{j}^{\prime}, \theta_{j}\right) \\
f_{3}\left(\theta_{j}\right) & :=C\left(\theta_{(1)}^{(j-1)} \wedge \theta_{(1)}^{(j-1)}, \theta_{j}, \theta_{(j+1)}^{(k)} \wedge \theta_{(j+1)}^{(k)}\right) g_{j}\left(x_{j}^{\prime}, \theta_{j}\right) \\
f_{4}\left(\theta_{j}\right) & :=C\left(\theta_{(1)}^{(j-1)} \vee \theta_{(1)}^{\prime(j-1)}, \theta_{j}, \theta_{(j+1)}^{(k)} \vee \theta_{(j+1)}^{\prime(k)}\right) g_{j}\left(x_{j}, \theta_{j}\right)
\end{aligned}
$$

e como $f_{1}(u) f_{2}(v) \leq f_{3}(u \vee v) f_{4}(u \wedge v)$ se, e somente se,

$C\left(\theta_{(1)}^{(j-1)}, u, \theta_{(j+1)}^{\prime(k)}\right) g_{j}\left(x_{j}, u\right) C\left(\theta_{(1)}^{(j-1)}, v, \theta_{(j+1)}^{(k)}\right) g_{j}\left(x_{j}^{\prime}, v\right)$

$\leq C\left(\theta_{(1)}^{(j-1)} \wedge \theta_{(1)}^{((j-1)}, u \vee v, \theta_{(j+1)}^{(k)} \wedge \theta_{(j+1)}^{(k)}\right) g_{j}\left(x_{j}^{\prime}, u \vee v\right)$

$C\left(\theta_{(1)}^{(j-1)} \vee \theta_{(1)}^{\prime(j-1)}, u \wedge v, \theta_{(j+1)}^{(k)} \vee \theta_{(j+1)}^{(k)}\right) g_{j}\left(x_{j}, u \wedge v\right)$

certo pelas hipóteses. Logo, pelos argumentos já empregados em provas semelhantes, verificamos (13); imediatamente vale (A.3).

○ Caso em que $X_{s}^{\prime}=X_{s}, s>j+1, X_{j+1}^{\prime}<X_{j+1}$. (A.3) é equivalente a

$$
\begin{aligned}
& g_{j+1}\left(x_{j+1}, \theta_{j+1} \vee \theta_{j+1}^{\prime}\right) g_{j+1}\left(x_{j+1}^{\prime}, \theta_{j+1} \wedge \theta_{j+1}^{\prime}\right) \\
& \int g_{j}\left(x_{j}, \theta_{j}\right) C\left(\theta_{(1)}^{j-1} \vee \theta_{(1)}^{(j-1)}, \theta_{j}, \theta_{(j+1)}^{(k)} \vee \theta_{(j+1)}^{(k)}\right) d \theta_{j} \\
& \int g_{j}\left(x_{j}^{\prime}, \theta_{j}\right) C\left(\theta_{(1)}^{(j-1)} \wedge \theta_{(1)}^{(j-1)}, \theta_{j}, \theta_{(j+1)}^{(k)} \wedge \theta_{(j+1)}^{(k)}\right) d \theta_{j} \\
& \geq g_{j+1}\left(x_{j+1}, \theta_{j+1}^{\prime}\right) \int g_{j}\left(x_{j}, \theta_{j}\right) C\left(\theta_{(1)}^{(j-1)}, \theta_{j}, \theta_{(j+1)}^{(k)}\right) d \theta_{j} \\
& g_{j+1}\left(x_{j+1}^{\prime}, \theta_{j+1}\right) \int g_{j}\left(x_{j}^{\prime}, \theta_{j}\right) C\left(\theta_{(1)}^{(k)}\right) d \theta_{j},
\end{aligned}
$$

pois as $g_{i}$, são $T P_{2}$. Mas, como $x_{j+1}^{\prime} \leq x_{j+1}$ e $g_{j+1}$ é $T P_{2}$ o problema se reduz á verificação de (13), já demonstrada. 


\section{Teorema B*}

Seja

$$
\xi^{*}(\underline{\theta} \mid \underline{X})=H(\underline{X}) \prod_{i=1}^{k} g_{i}\left(X_{i}, \theta_{i}\right) C(\underline{\theta}) .
$$

Suponhamos ainda, que $\exists j$, tal que

$$
g_{j} \text { é } R R_{2}\left(X_{j}, \theta_{j}\right), g_{i} \text { é } T P_{2}\left(X_{i}, \theta_{i}\right) \forall i \neq j, i=1, \cdots, k \text {. }
$$

E seja $C, M T P_{2}(\underline{\theta})$.

Então, se

$$
\underline{X}=\left(X_{1}, \cdots, X_{j-1}, X_{j}, X_{j+1}, \cdots, X_{k}\right), \underline{X^{\prime}}=\left(X_{1}^{\prime}, \cdots, X_{j-1}^{\prime}, X_{j}^{\prime}, X_{j+1}^{\prime}, \cdots, X_{k}^{\prime}\right)
$$

$\operatorname{com} X_{i}^{\prime} \leq X_{i} \forall i \neq j, X_{j}^{\prime} \geq X_{j}$

$$
\xi^{*}(\underline{\theta} \mid \underline{X})>_{T P_{2}(\underline{\theta})} \xi^{*}\left(\underline{\theta} \mid \underline{X^{\prime}}\right)
$$

Prova: notemos que

$$
\text { (B.1) } \xi^{*}(\underline{\theta} \mid \underline{X})>_{T P_{2}(\underline{\theta})} \xi^{*}\left(\underline{\theta} \mid \underline{X^{\prime}}\right)
$$

é equivalente a (B.2)

$$
\begin{aligned}
& k \quad k \\
& \prod g_{i}\left(x_{i}, \theta_{i} \vee \theta_{i}^{\prime}\right) \quad \prod \quad g_{i}\left(x_{i}^{\prime}, \theta_{i} \wedge \theta_{i}^{\prime}\right) \\
& i \neq j \quad i \neq j \\
& g_{j}\left(x_{j}, \theta_{j} \vee \theta_{j}^{\prime}\right) g_{j}\left(x_{j}^{\prime}, \theta_{j} \wedge \theta_{j}^{\prime}\right) C\left(\underline{\theta} \vee \underline{\theta}^{\prime}\right) C\left(\underline{\theta} \wedge \underline{\theta}^{\prime}\right) \\
& \geq g_{j}\left(x_{j}, \theta_{j}^{\prime}\right) \prod_{\substack{i=1 \\
i \neq j}}^{k} g_{i}\left(x_{i}, \theta_{i}^{\prime}\right) g_{j}\left(x_{j}^{\prime}, \theta_{j}\right) \prod_{\substack{i=1 \\
i \neq j}}^{k} g_{i}\left(x_{i}^{\prime}, \theta_{i}\right) C\left(\underline{\theta}^{\prime}\right) C(\underline{\theta}),
\end{aligned}
$$


como $g_{i}$ é $T P_{2}\left(X_{i}, \theta_{i}\right)$ e $x_{i}^{\prime} \leq x_{i}, \forall i \neq j$, (B.2) é equivalente a

$g_{j}\left(x_{j}, \theta_{j} \vee \theta_{j}^{\prime}\right) C\left(\underline{\theta} \vee \underline{\theta}^{\prime}\right) g_{j}\left(x_{j}^{\prime}, \theta_{j} \wedge \theta_{j}^{\prime}\right) C\left(\underline{\theta} \wedge \underline{\theta^{\prime}}\right) \geq g_{j}\left(x_{j}, \theta_{j}^{\prime}\right) C\left(\underline{\theta}^{\prime}\right) g_{j}\left(x_{j}^{\prime}, \theta_{j}\right) C(\underline{\theta})$,

que resulta direto das hipóteses, $x_{j}^{\prime} \geq x_{j}, g_{j} R R_{2}$ e $C, M T P_{2}$.

\section{Teorema $\mathrm{C}^{*}$}

$$
\xi^{*}(\underline{\theta} \mid \underline{X})=H(\underline{X}) \prod_{i=1}^{k} g_{i}\left(X_{i}, \theta_{i}\right) C(\underline{\theta})
$$

$g_{i}$ é $R R_{2}\left(X_{i}, \theta_{i}\right) \forall i=1, \cdots, j, g_{i}$ é $T P_{2}\left(X_{i}, \theta_{i}\right) \forall i=j+1, \cdots, k$. C é $M T P_{2}(\underline{\theta})$.

Então, se

$$
\underline{X}=\left(X_{1}, \cdots, X_{j-1}, X_{j}, X_{j+1}, \cdots, X_{k}\right) ; \underline{X^{\prime}}=\left(X_{1}^{\prime}, \cdots, X_{j-1}^{\prime}, X_{j}^{\prime}, X_{j+1}^{\prime}, \cdots, X_{k}^{\prime}\right)
$$

$\operatorname{com} X_{i}^{\prime} \geq X_{i} \forall i=1, \cdots, j$ e $X_{i}^{\prime} \leq X_{i} \forall i=j+1, \cdots, k$,

$$
\xi^{*}(\underline{\theta} \mid \underline{X})>_{T P_{2}(\underline{\theta})} \xi^{*}\left(\underline{\theta} \mid \underline{X^{\prime}}\right) .
$$

Prova: Como $g_{i}$ é $R R_{2}\left(x_{i}, \theta_{i}\right)$ e $x_{i}^{\prime} \geq x_{i}, i=1, \cdots, j$,

$$
g_{i}\left(x_{i}, \theta_{i} \vee \theta_{i}^{\prime}\right) g_{i}\left(x_{i}^{\prime}, \theta_{i} \wedge \theta_{i}^{\prime}\right) \geq g_{i}\left(x_{i}, \theta_{i}^{\prime}\right) g_{i}\left(x_{i}^{\prime}, \theta_{i}\right)
$$

logo,

$$
\text { (a) } \prod_{i=1}^{j} g_{i}\left(x_{i}, \theta_{i} \vee \theta_{i}^{\prime}\right) \prod_{i=1}^{j} g_{i}\left(x_{i}^{\prime}, \theta_{i} \wedge \theta_{i}^{\prime}\right) \geq \prod_{i=1}^{j} g_{i}\left(x_{i}, \theta_{i}^{\prime}\right) \prod_{i=1}^{j} g_{i}\left(x_{i}^{\prime}, \theta_{i}\right) \text {. }
$$

Como $g_{i}$ é $T P_{2}\left(x_{i}, \theta_{i}\right)$ e $x_{i}^{\prime} \leq x_{i}, \quad i=j+1, \cdots, k$. Logo,

$$
\text { (b) } \prod_{i=j+1}^{k} g_{i}\left(x_{i}, \theta_{i} \vee \theta_{i}^{\prime}\right) \prod_{i=j+1}^{k} g_{i}\left(x_{i}^{\prime}, \theta_{i} \wedge \theta_{i}^{\prime}\right) \geq \prod_{i=j+1}^{k} g_{i}\left(x_{i}, \theta_{i}^{\prime}\right) \prod_{i=j+1}^{k} g_{i}\left(x_{i}^{\prime}, \theta_{i}\right)
$$


e

$$
\text { (c) } C\left(\underline{\theta} \wedge \underline{\theta}^{\prime}\right) C\left(\underline{\theta} \vee \underline{\theta}^{\prime}\right) \geq C(\underline{\theta}) C\left(\underline{\theta}^{\prime}\right)
$$

$(a),(b),(c)$ além da forma da posteriori permitem concluir a prova.

\section{Exemplo : Distribuição Normal Multivariada}

Seja $\underline{X}$ um vetor aleatório $k$-dimensional, com coordenadas $X_{i}, i=1, \cdots, k$ independentes. Suponhamos que para todo $i, \theta_{i}:=E\left(X_{i}\right)$ é desconhecida, enquanto que $\sigma_{i}:=\operatorname{Var}\left(X_{i}\right)$ é conhecida. Assim,

$$
\begin{aligned}
f(\underline{X} \mid \underline{\theta}) & =|\Sigma|^{-\frac{1}{2}} \exp \left(\frac{-(\underline{X}-\underline{\theta})^{t}|\Sigma|^{-1}(\underline{X}-\underline{\theta})}{2}\right) \\
& =h(\underline{X}) g(\underline{X}, \underline{\theta}) \\
h(\underline{X}) & :=\prod_{i=1}^{k} \exp \frac{-X_{i}^{2}}{2 \sigma_{i}^{2}} \\
g(\underline{X}, \underline{\theta}) & :=\prod_{i=1}^{k} \exp \frac{X_{i} \theta_{i}}{\sigma_{i}^{2}} \prod_{i=1}^{k} \exp \frac{-\theta_{i}^{2}}{2 \sigma_{i}^{2}}
\end{aligned}
$$

Afirmação: $h$ é $M T P_{2}(\underline{X})$ e $g$ é $M T P_{2}(\underline{X}, \underline{\theta})$.

Os delineamentos para a prova de: $g M T P_{2}(\underline{X}, \underline{\theta})$ são os seguintes,

$$
\begin{aligned}
g(\underline{X}, \underline{\theta}) & =\prod_{i=1}^{k} g_{i}\left(X_{i}, \theta_{i}\right) D(\underline{\theta}), \\
g_{i}\left(X_{i}, \theta_{i}\right) & =\exp \frac{X_{i} \theta_{i}}{\sigma_{i}^{2}} \\
D(\underline{\theta}) & :=\prod_{i=1}^{k} D_{i}\left(\theta_{i}\right) \\
D_{i}\left(\theta_{i}\right) & :=\exp \frac{-\theta_{i}^{2}}{2 \sigma_{i}^{2}} .
\end{aligned}
$$


$D(\underline{\theta})$ é $T P_{2}$ em pares, enquanto que $\prod_{i=1}^{k} g_{i}\left(X_{i}, \theta_{i}\right)$ é $T P_{2}$ nos pares

$$
\left(X_{s}, X_{j}\right),\left(\theta_{s}, \theta_{j}\right),\left(X_{s}, \theta_{j}\right), j \neq s
$$

logo, $g(\underline{X}, \underline{\theta})$ é $T P_{2}$ em pares, mas como em nosso caso $g(\underline{X}, \underline{\theta}) \neq 0$, por 1.3 .3 resulta $g, M T P_{2}$. Usando a mesma proposição, prova-se que $h$ é $M T P_{2}$.

Aplicação dos Teoremas $\mathrm{A}^{*}$ e $\mathbf{B}^{*}$

A expressão da posteriori fica,

$$
\begin{aligned}
\xi^{*}(\underline{\theta} \mid \underline{X}) & =S(\underline{X}) \prod_{i=1}^{k} g_{i}\left(X_{i}, \theta_{i}\right) C(\underline{\theta}) \\
C(\underline{\theta}) & :=\xi(\underline{\theta}) \prod_{i=1}^{k} \exp \frac{-\theta_{i}^{2}}{2 \sigma_{i}^{2}} \\
S(\underline{X}) & :=h(\underline{X})\left(\int f(\underline{X} \mid \underline{\theta}) \xi(\underline{\theta}) d \underline{\theta}\right)^{-1}
\end{aligned}
$$

Assim, verificar (C) (do Teorema $\mathrm{A}^{*}$ ) sobre $C$ equivale a verificar (C) sobre $\xi$. Assumimos como priori para $\underline{\theta}$ uma Normal de vetor de médias $\underline{0}$ e matriz de covariâncias dada pela identidade $I$,

$$
\xi(\underline{\theta}) \propto \prod_{i=1}^{k} \exp \frac{-\theta_{i}^{2}}{2}, \text { que verifica (C). }
$$




\section{Referências}

Ash, R.B. (1972). Real Analysis and Probability. Probability and Mathematical Statistics. A Series of Monographs and Text books. University of Illionois. Academic Press. New York and London.

Barlow, R. E.; Proschan, F. (1981). Statistical Theory of reliability and life testing. Silver Spring, MD.

Basu, D.; Cheng, C. (1981). A note on the notion of adequacy.

Block H.W.; Savits T.H.; Shaked M. (1982). Some concepts of negative dependence, Ann.Probab. 10, 765-772.

Boland, P. J.; Proschan, F. (1983). The reliability of K out of $\mathrm{N}$ systems. The Annals of Probability, Vol. 11, 3, 760-764.

Boland, P. J.; Proschan, F.; Tong, Y.L. (1988). Moment and geometric Probability inequalities arising from arrangement increasing functions. The Annals of Probability Vol. 16, 1, 407-413.

Boland, P. J.; Proschan, Frank; Tong, Y. L. (1989). Modelling dependence in simple and indirect majority systems. J.Appl.Prob. Vol. 26, 81-88.

Boland, P. J.; Hollander, M.; Joag-Dev, K.; Kochar, S. (1996). Bivariate dependence properties of order statistics. J. Multivariate Anal. Vol. 56, 1, 75-89. 
Brow, M.; Proschan, F.(1983). Imperfect repair. J. Appl.Prob. Vol. 20, 851-859.

De Finetti, B. (1953). Trasformazioni di numeri aleatori atte a far coincidere distribuzioni diverse. Istituto Italiano Degli Attuari-Anno XVI 51-57.

Ebrahimi, N.; Ghosh, M. (1981). Multivariate Negative Dependence. Communications in statistics (Theory and methods.) A10, 4, 307-337.

Efron P. (1965). Increasing properties of Pólya frequency functions. Ann.Math.Statist. $36,272-279$.

Esteves, L.G. (1997). Coincidência de distribuições através de uma transformação de De Finetti. Disertação de Mestrado-IME. USP.

Fahmy, S.; C.A.de B.Pereira; Proschan, F.; Shaked, M. (1982). The influence of the sample on the posterior distribution. Communications in statistics (Theory and methods.) Vol.11, 16, 1757-1768.

Fréchet, M. (1951). Sur les tableaux de corrélation dont les margens sont données. Annales de L' Université de Lyon Sect, A Ser 3, 14, 53-77.

Genest, CH.; MacKay, J. (1986). The Joy of Copulas: Bivariate Distributions With Uniform Marginals. The American Statistician Vol.40, 4, 280-283.

Joag-Dev, K. (1983). Independence via uncorrelatedness under certain dependence structures. Ann. Probab. Vol. 11, 4, 1037-1041.

Joag-Dev, K.; Perlman, M. D.; Pitt, L. D.(1983). Association of normal random variables and Slepian's inequality. The Annals of Probability Vol. 11, 2, 451455. 
Joag-Dev, K.; Proschan, F. (1983). Negative association of random variables, with applications. Ann. Statist.Vol. 11, 1, 286-295.

Joag-Dev, K.; Kochar, S.; Proschan, F. (1995). A general composition theorem and its applications to certain partial orderings of distributions. Statist. Probab. Lett. Vol. 22, 2, 111-119.

Joe, H. (1997). Multivariate Models and Dependence Concepts. Monographs on Statistics and Applied Probability 73 (Chapman and Hall).

Karlin, S. (1968). Total Positivity. Stanford University Press. Stanford, California.

Karlin, S.; Rinott, Y. (1980 a). Classes of ordering of measures and related correlation inequalities.I. Multivariada totaly positive distributions. J. Multivariate Anal. Vol.10, 4, 467-498.

Karlin, S.; Rinott, Y. (1980 b). Classes of ordering of measures and related correlation inequalities.II.Multivariada reverse rule distributions. J. Multivariate Anal. Vol.10, 4, 499-518.

Kim, J. S.; Proschan, F.(1995). A review: the arrangement increasing partial ordering. Comput. Oper. Res. Vol 22, 4, 357-371.

Kruskal, W. H. (1958). Ordinal measures of association. American Statistical Association Journal 53, 814-861.

Lehmann, E.L. (1966). Some concepts of dependence. Ann.Math.Statist. 43, 1137-1153. 
Li H.; Scarsini M.; Shaked M. (1996). A tool for the construction of multivariate distributions with given nonoverlapping multivariate marginals. J. Multivariate Anal. Vol 56, 1, 20-41.

Loéve, M.(1963). Probability Theory (Third Edition). Van Nostrand, New York.

Mikusinski, P.; Sherwood, H.; Taylor, M. D. (1991-1992). The Fréchet Bounds Revisited. Real Analysis Exchange Vol.17, 759-764.

Nelsen, R.B. (1992). On measures of association as measures of positive dependence. Statist. Probab. Lett, 14, 269-274.

Nelsen, R.B. (1995). Copulas, Characterization, Correlation, and Counterexamples. Mathematics Magazine Vol.68, 3, 193-198.

Skibinsky, M. (1967). Adequate Subfields and Sufficiency. Ann. Math. Statist Vol.38, 155-161.

Sklar, A. (1959). Fonctions de répartition à $n$ dimensions et leurs margens. Publications Institute des Statistics, Université de Paris, 8, 229-231.

Sklar, A. (1973). Random Variables, Join Distribution Function, and Copulas. Kibernetika Vol. 9, 6. 


\section{Tipos de dependências}

Barlow, R. E.; Proschan, F.(1981). Statistical Theory of reliability and life testing. Silver Spring, MD.

Block H.W.; Savits T.H.; Shaked M. (1982). Some concepts of negative dependence. Ann.Probab. 10, 765-772.

Joag-Dev, K.; Proschan, F. (1983). Negative association of random variables, with applications. Ann. Statist.Vol. 11, 1, 286-295.

Joe, H. (1997). Multivariate Models and Dependence Concepts. Monographs on Statistics and Applied Probability 73 (Chapman and Hall).

Karlin, S.; Rinott, Y. (1980 a). Classes of ordering of measures and related correlation inequalities.I. Multivariada totaly positive distributions. J. Multivariate Anal. Vol.10, 4, 467-498.

Karlin, S.; Rinott, Y. (1980 b). Classes of ordering of measures and related correlation inequalities.II.Multivariada reverse rule distributions. J. Multivariate Anal. Vol.10, 4, 499-518. 


\section{Acoplamentos e Classes de Fréchet}

Fréchet, M. (1951). Sur les tableaux de corrélation dont les margens sont données. Annales de L'Université de Lyon Sect, A Ser 3, 14, 53-77.

Genest, CH.; MacKay. J. (1986). The Joy of Copulas: Bivariate Distributions With Uniform Marginals. The American Statistician Vol.40, 4, 280-283.

Joe, H. (1997). Multivariate Models and Dependence Concepts. Monographs on Statistics and Applied Probability 73 (Chapman and Hall).

Mikusinski, P.; Sherwood, H.; Taylor, M. D. (1991-1992). The Fréchet Bounds Revisited. Real Analysis Exchange Vol.17, 759-764.

Nelsen, R.B. (1992). On measures of association as measures of positive dependence. Statist. Probab. Lett, 14, 269-274.

Nelsen, R.B. (1995). Copulas, Characterization, Correlation, and Counterexamples. Mathematics Magazine Vol.68, 3, 193-198.

Sklar, A. (1959). Fonctions de répartition à $n$ dimensions et leurs margens. Publications Institute des Statistics, Université de Paris, 8, 229-231.

Sklar, A. (1973). Random Variables, Joint Distribution Function, and Copulas. Kibernetika Vol. 9, 6. 


\section{Indice de Tópicos}

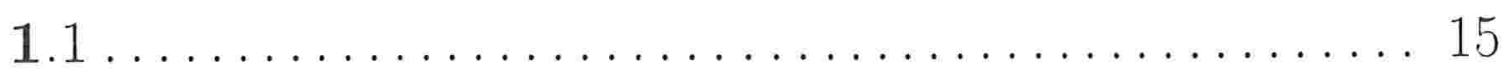

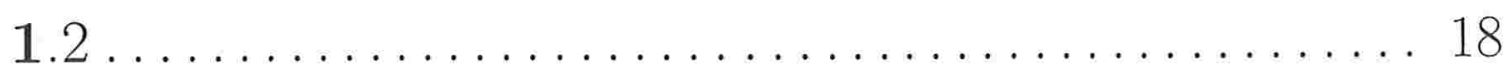

$1.2 .1 \ldots \ldots \ldots \ldots \ldots \ldots \ldots \ldots \ldots \ldots \ldots \ldots \ldots, 18$

$1.2 .2 \ldots \ldots \ldots \ldots \ldots \ldots \ldots \ldots \ldots \ldots \ldots$

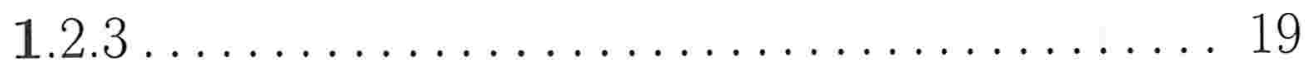

$1.2 .4 \ldots \ldots \ldots \ldots \ldots \ldots \ldots \ldots \ldots \ldots \ldots \ldots \ldots \ldots \ldots, 20$

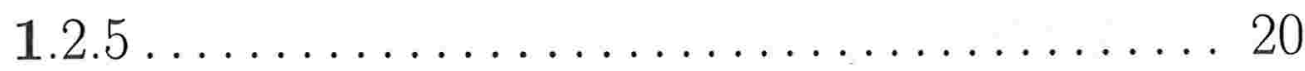

$1.2 .6 \ldots \ldots \ldots \ldots \ldots \ldots \ldots \ldots \ldots \ldots \ldots \ldots \ldots \ldots \ldots$

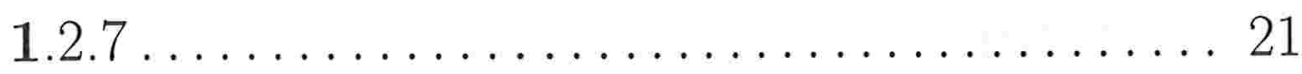

$1.2 .8 \ldots \ldots \ldots \ldots \ldots \ldots \ldots \ldots \ldots \ldots \ldots \ldots \ldots \ldots \ldots$

$1.2 .9 \ldots \ldots \ldots \ldots \ldots \ldots \ldots \ldots \ldots \ldots \ldots \ldots \ldots \ldots \ldots \ldots, 22$

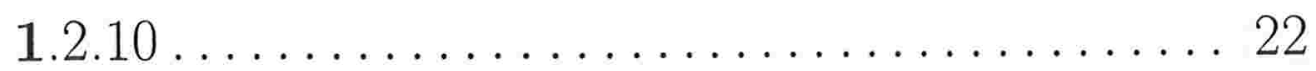

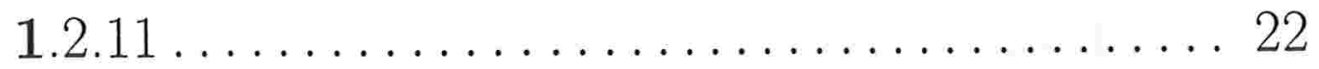


$1.2 .12 \ldots \ldots \ldots \ldots \ldots \ldots \ldots \ldots \ldots \ldots \ldots \ldots$

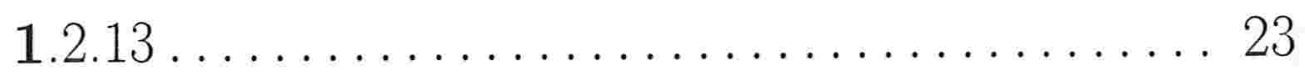

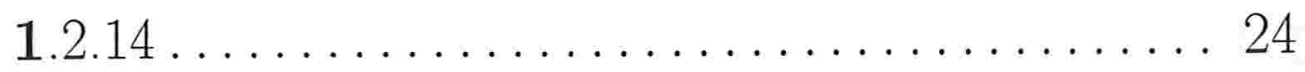

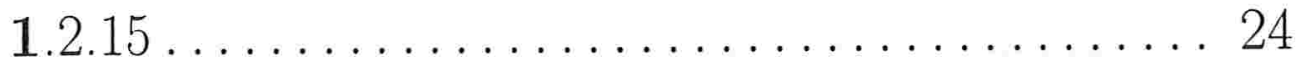

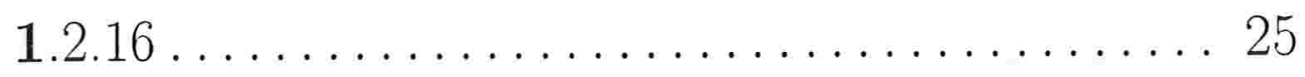

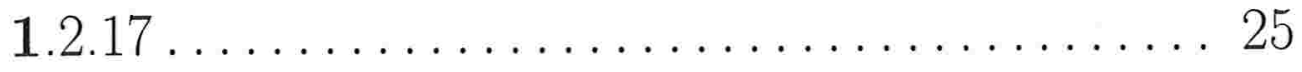

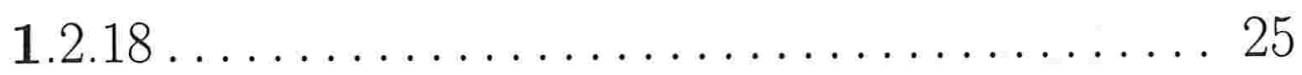

$1.2 .19 \ldots \ldots \ldots \ldots \ldots \ldots \ldots \ldots \ldots \ldots \ldots \ldots$

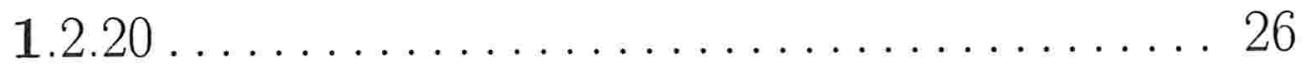

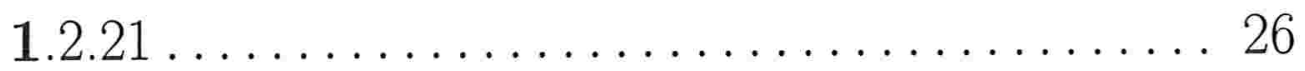

$1.2 .22 \ldots \ldots \ldots \ldots \ldots \ldots \ldots \ldots \ldots \ldots \ldots \ldots \ldots$

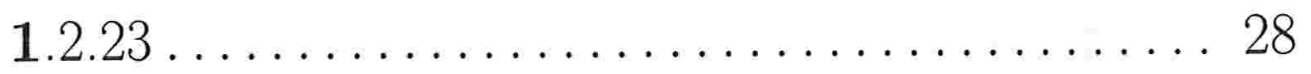

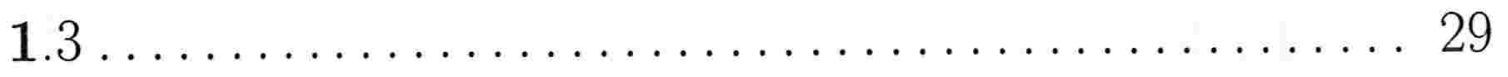

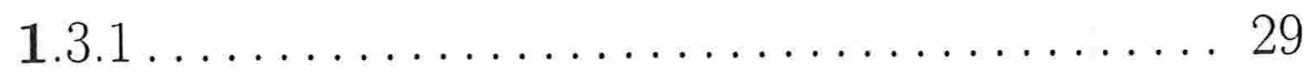




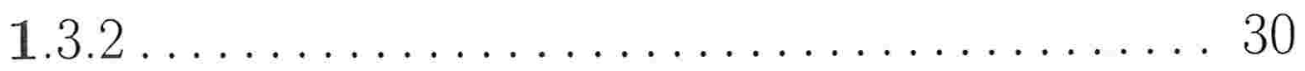

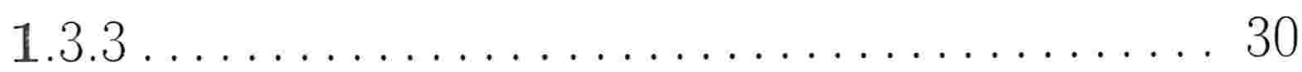

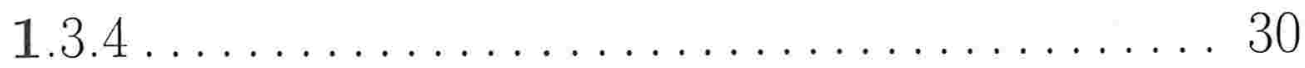

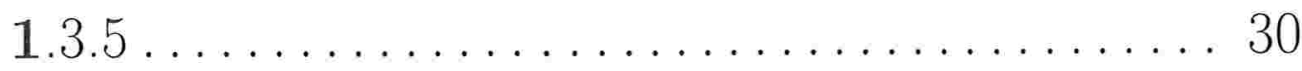

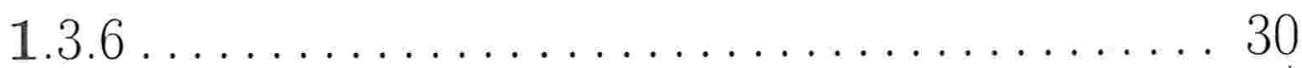

$1.3 .7 \ldots \ldots \ldots \ldots \ldots \ldots \ldots \ldots \ldots \ldots \ldots \ldots \ldots$

$1.3 .8 \ldots \ldots \ldots \ldots \ldots \ldots \ldots \ldots \ldots \ldots \ldots \ldots \ldots$

$1.3 .9 \ldots \ldots \ldots \ldots \ldots \ldots \ldots \ldots \ldots \ldots \ldots \ldots \ldots$

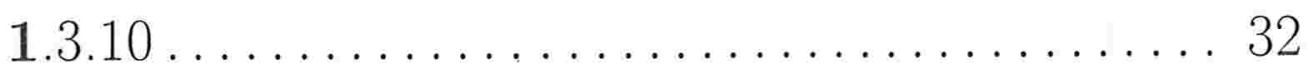

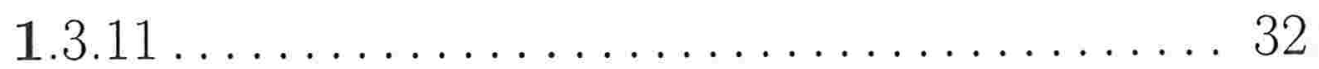

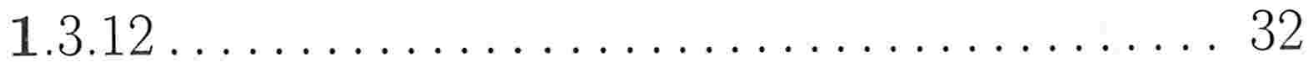

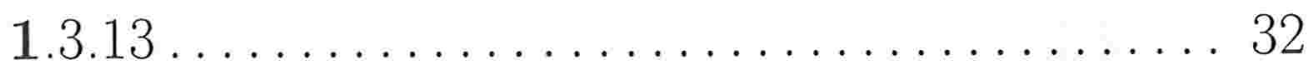

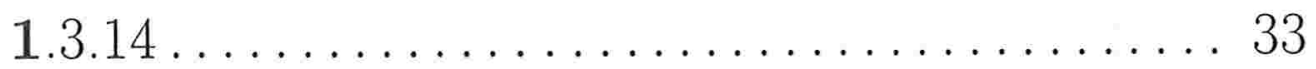

$1.3 .15 \ldots \ldots \ldots \ldots \ldots \ldots \ldots \ldots \ldots \ldots \ldots \ldots$ 


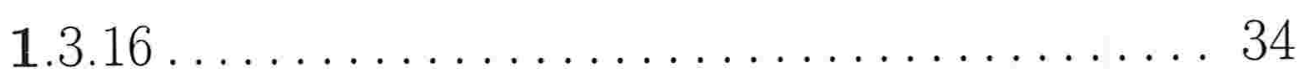

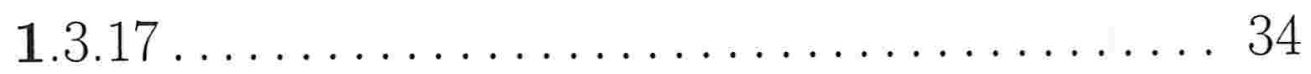

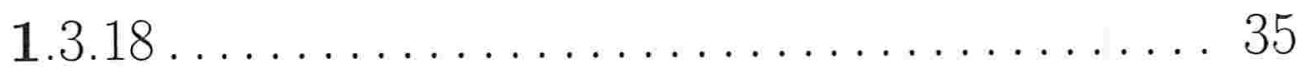

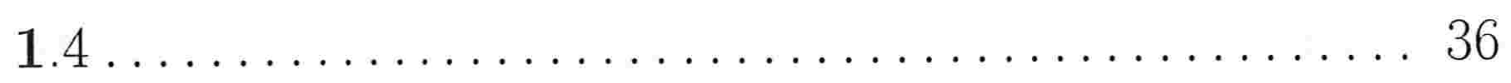

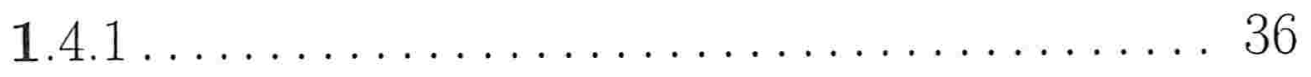

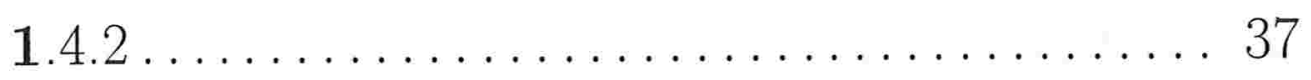

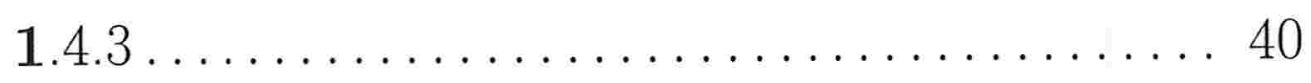

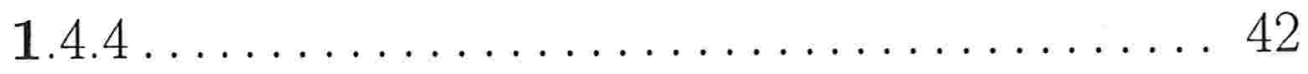

$1.4 .5 \ldots \ldots \ldots \ldots \ldots \ldots \ldots \ldots \ldots \ldots \ldots \ldots$

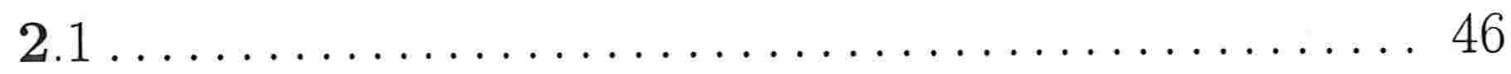

$2.1 .1 \ldots \ldots \ldots \ldots \ldots \ldots \ldots \ldots \ldots \ldots, 46$

$2.1 .2 \ldots \ldots \ldots \ldots \ldots \ldots \ldots \ldots \ldots \ldots \ldots \ldots$

$2.1 .3 \ldots \ldots \ldots \ldots \ldots \ldots \ldots \ldots \ldots \ldots \ldots \ldots$

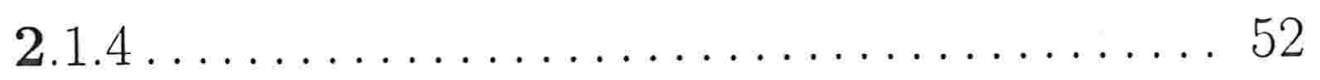




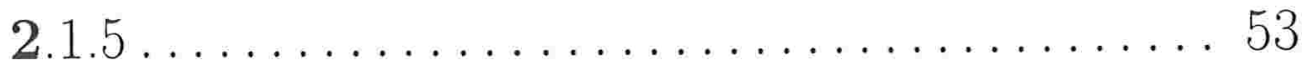

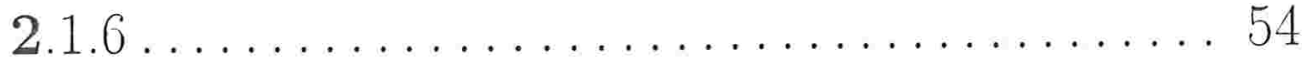

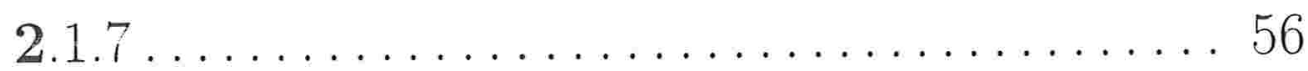

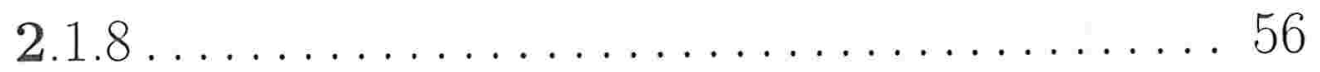

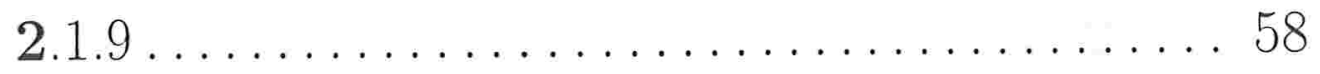

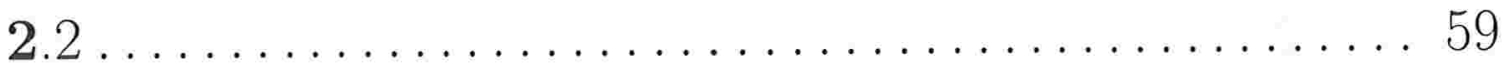

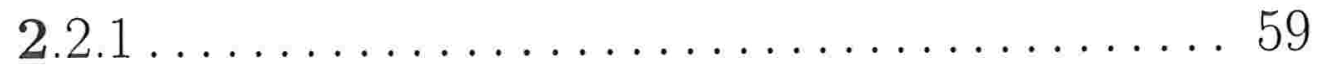

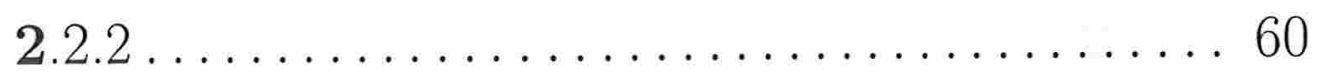

$2.2 .3 \ldots \ldots \ldots \ldots \ldots \ldots \ldots \ldots \ldots \ldots \ldots \ldots \ldots \ldots \ldots \ldots$

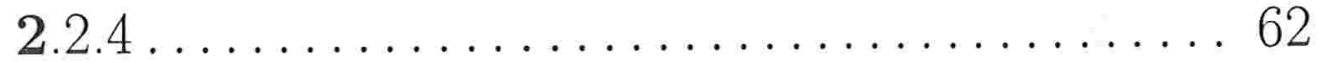

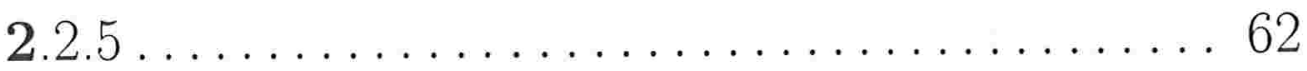

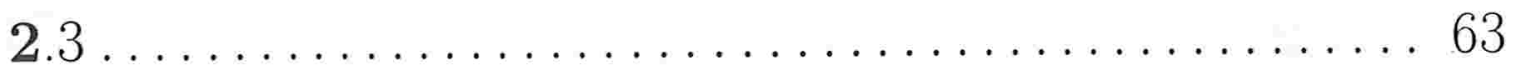

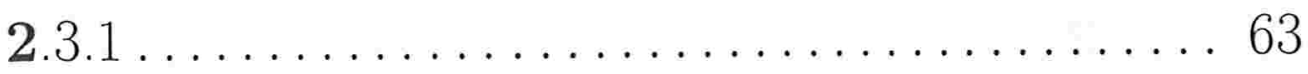

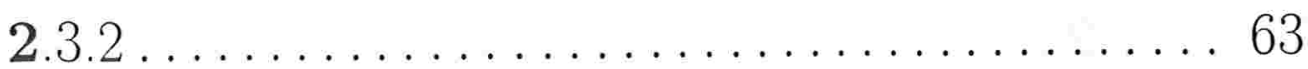




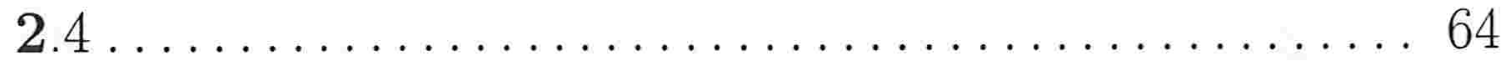

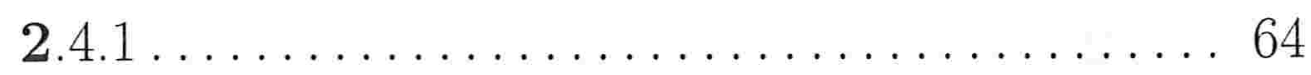

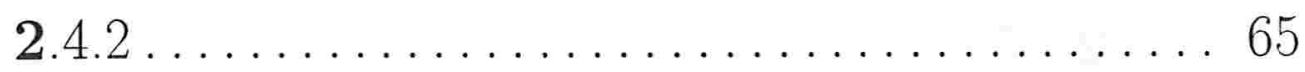

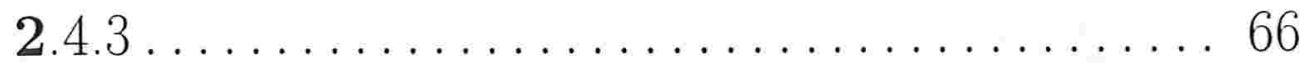

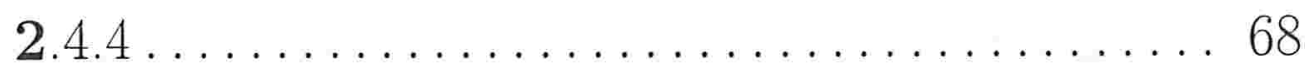

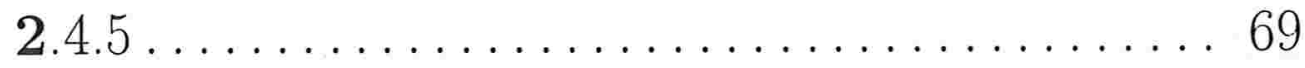

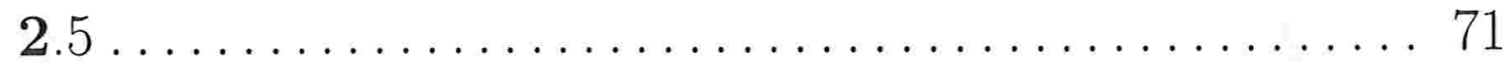

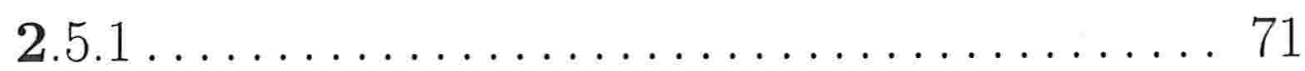

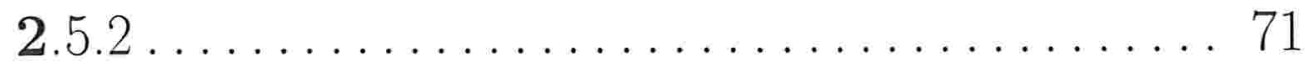

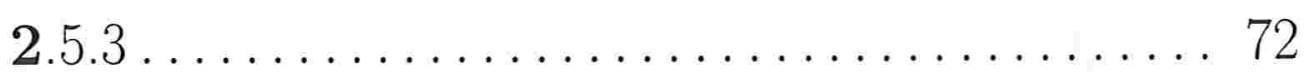

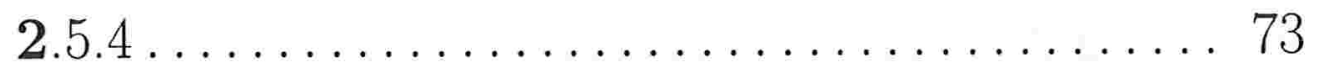

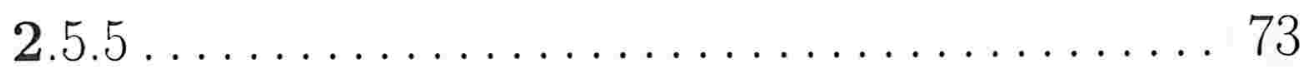

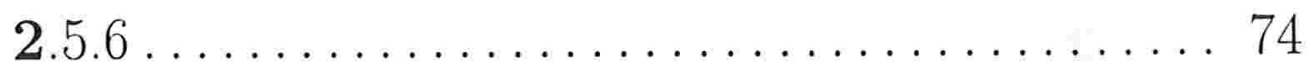

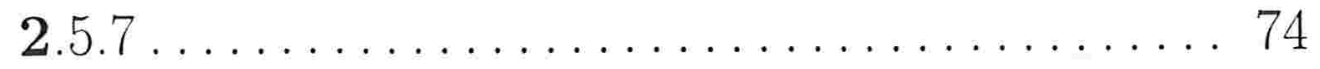




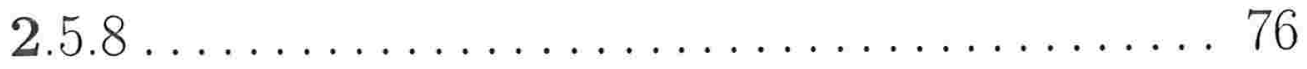

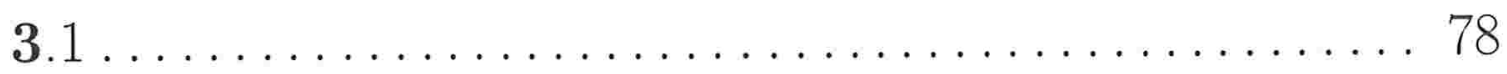

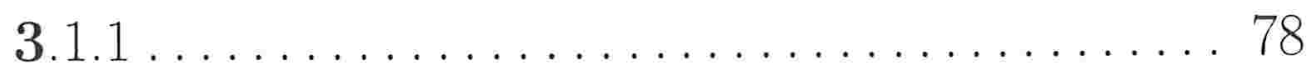

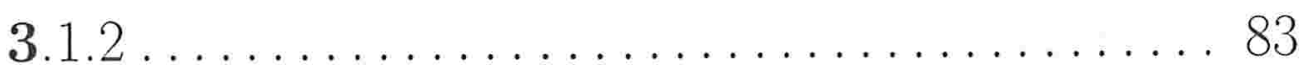

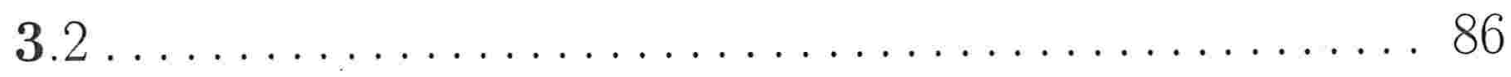

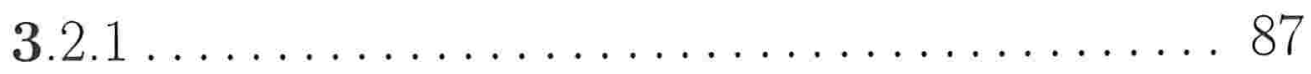

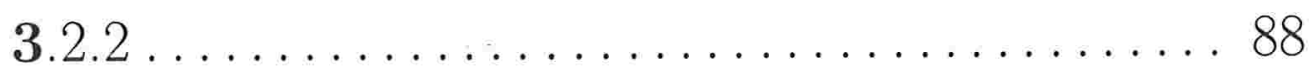

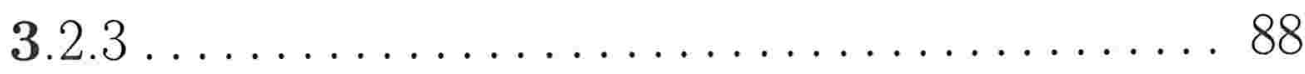

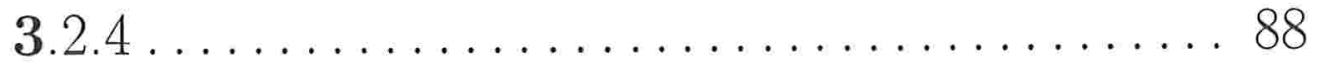

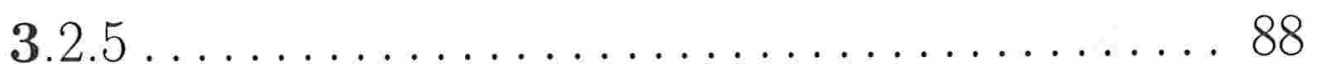

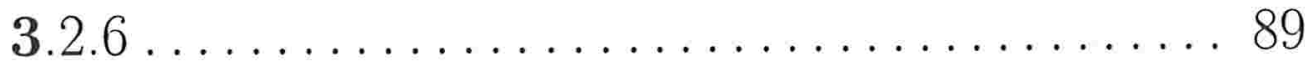

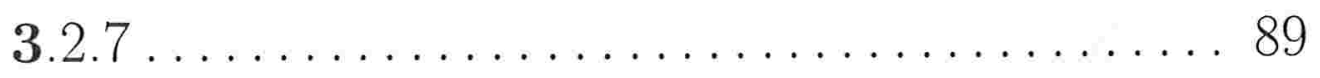

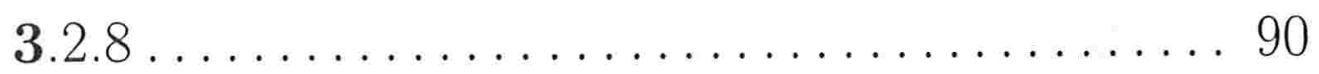

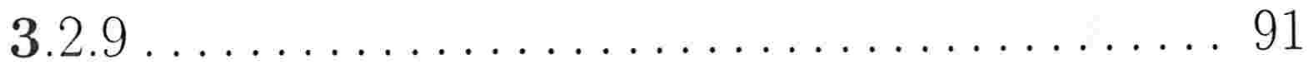




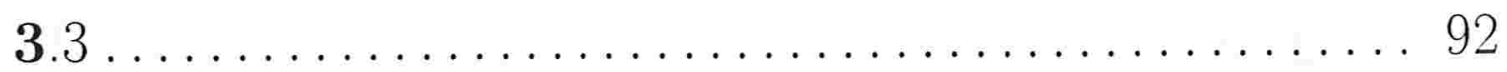

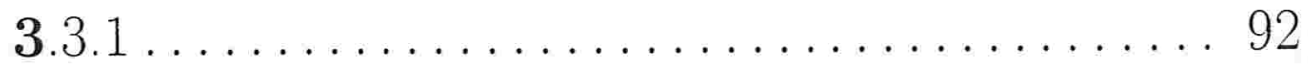

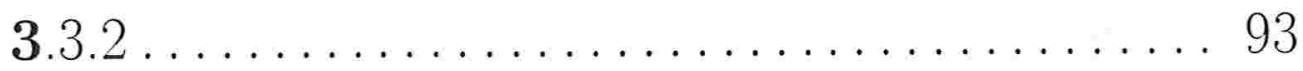

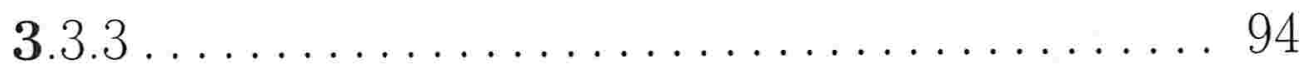

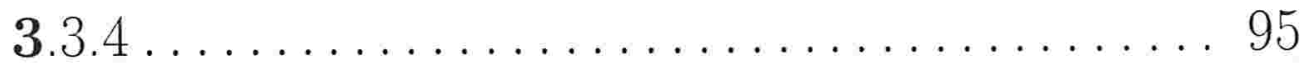

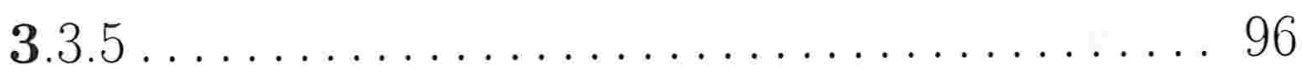

$3.3 .6 \ldots \ldots \ldots \ldots \ldots \ldots \ldots \ldots \ldots \ldots$

$3.3 .7 \ldots \ldots \ldots \ldots \ldots \ldots \ldots \ldots \ldots \ldots \ldots$

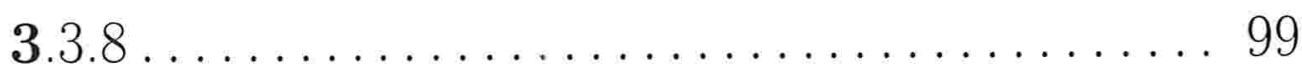

$3.3 .9 \ldots \ldots \ldots \ldots \ldots \ldots \ldots \ldots \ldots \ldots \ldots \ldots \ldots \ldots \ldots \ldots$

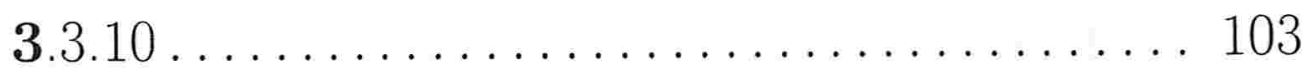

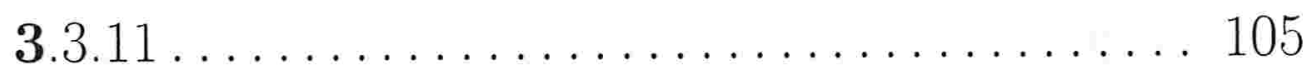

$3.3 .12 \ldots \ldots \ldots \ldots \ldots \ldots \ldots \ldots \ldots \ldots \ldots \ldots \ldots$

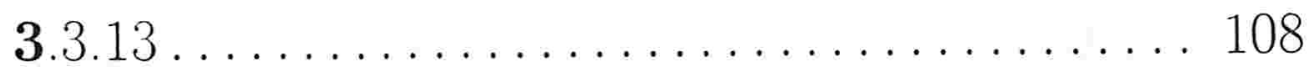




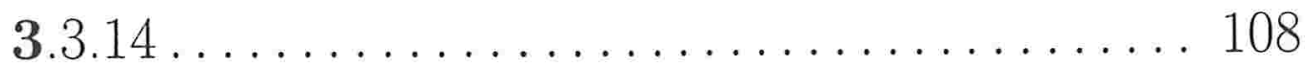

$3.3 .15 \ldots \ldots \ldots \ldots \ldots \ldots \ldots \ldots \ldots \ldots \ldots$

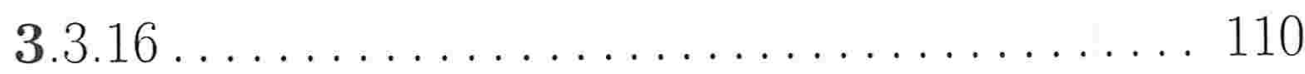

$3.3 .17 \ldots \ldots \ldots \ldots \ldots \ldots \ldots \ldots \ldots \ldots \ldots \ldots$

$3.3 .18 \ldots \ldots \ldots \ldots \ldots \ldots \ldots \ldots \ldots \ldots \ldots \ldots \ldots$

$3.3 .19 \ldots \ldots \ldots \ldots \ldots \ldots \ldots \ldots \ldots \ldots \ldots \ldots \ldots$

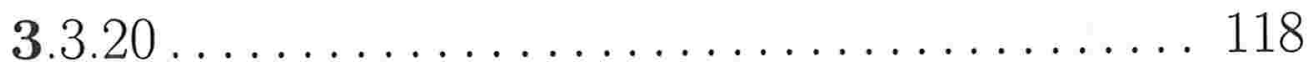

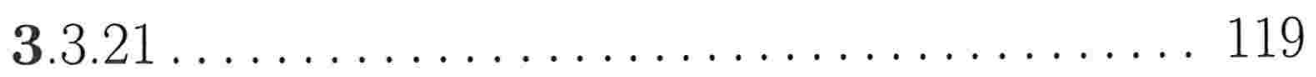

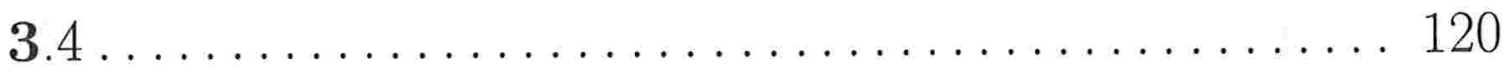

$3.4 .1 \ldots \ldots \ldots \ldots \ldots \ldots \ldots \ldots \ldots \ldots \ldots \ldots \ldots \ldots \ldots$

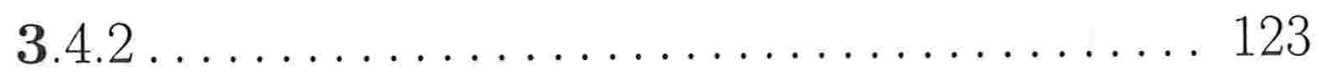

$3.4 .3 \ldots \ldots \ldots \ldots \ldots \ldots \ldots \ldots \ldots \ldots \ldots \ldots \ldots \ldots \ldots$

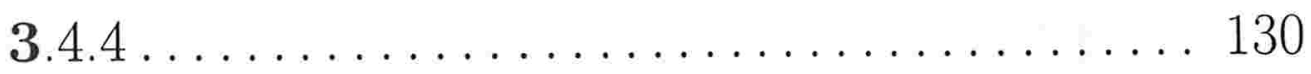

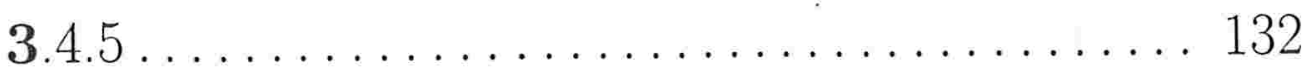


236

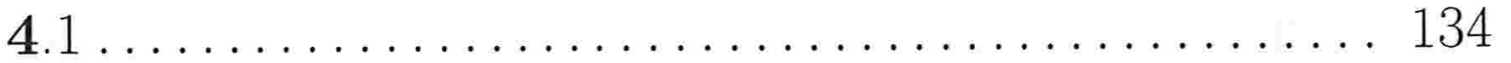

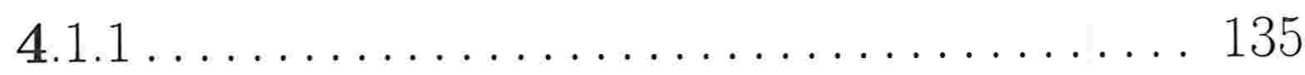

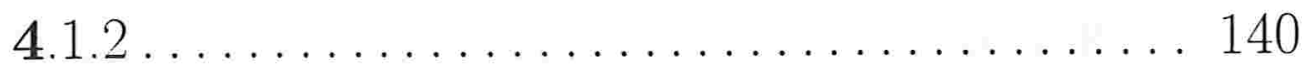

$4.1 .3 \ldots \ldots \ldots \ldots \ldots \ldots \ldots \ldots \ldots \ldots \ldots \ldots \ldots$

$4.1 . \ldots \ldots \ldots \ldots \ldots \ldots \ldots \ldots \ldots \ldots \ldots \ldots \ldots$

$4.1 .5 \ldots \ldots \ldots \ldots \ldots \ldots \ldots \ldots \ldots \ldots \ldots \ldots \ldots$

$4.1 .6 \ldots \ldots \ldots \ldots \ldots \ldots \ldots \ldots \ldots \ldots \ldots \ldots \ldots \ldots$

$4.1 .7 \ldots \ldots \ldots \ldots \ldots \ldots \ldots \ldots \ldots \ldots \ldots \ldots \ldots$

$4.1 .8 \ldots \ldots \ldots \ldots \ldots \ldots \ldots \ldots \ldots \ldots \ldots \ldots \ldots$

$4.1 .9 \ldots \ldots \ldots \ldots \ldots \ldots \ldots \ldots \ldots \ldots \ldots \ldots \ldots$

$4.110 \ldots \ldots \ldots \ldots \ldots \ldots \ldots \ldots \ldots \ldots \ldots$

$4.1 .11 \ldots \ldots \ldots \ldots \ldots \ldots \ldots \ldots \ldots \ldots \ldots \ldots$

$4.1 .12 \ldots \ldots \ldots \ldots \ldots \ldots \ldots \ldots \ldots \ldots \ldots \ldots$

$4.1 .13 \ldots \ldots \ldots \ldots \ldots \ldots \ldots \ldots \ldots \ldots \ldots \ldots$ 


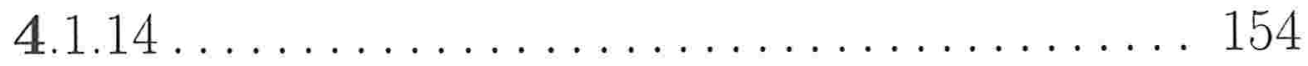

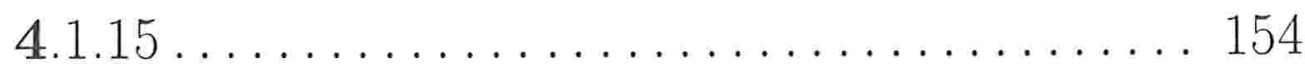

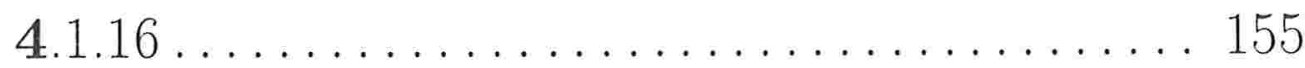

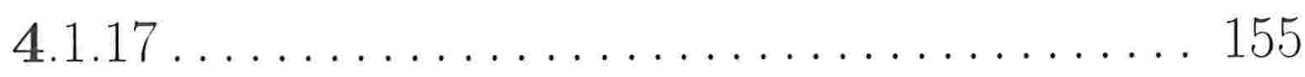

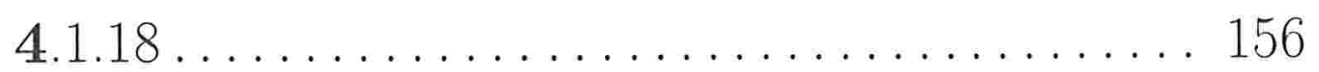

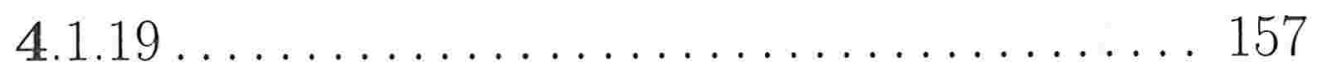

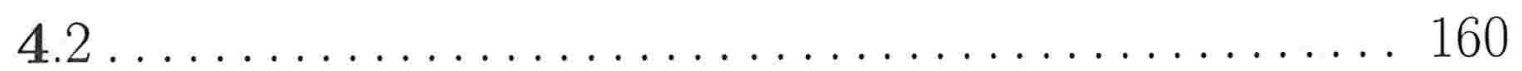

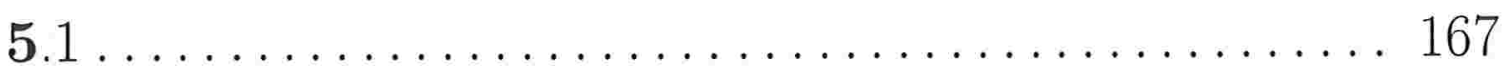

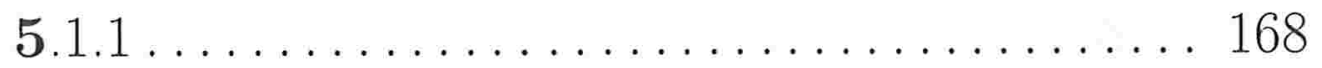

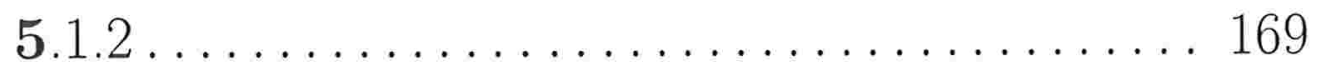

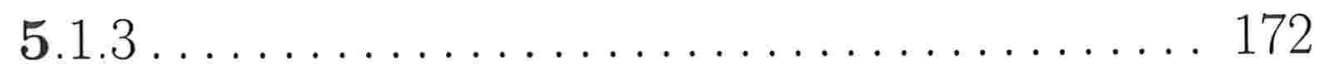

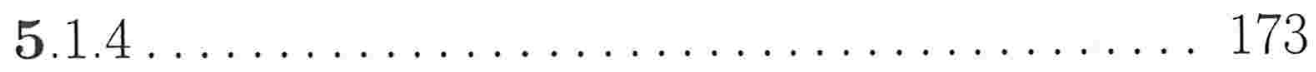

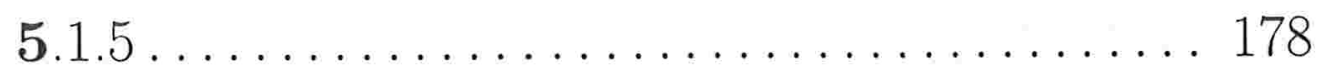

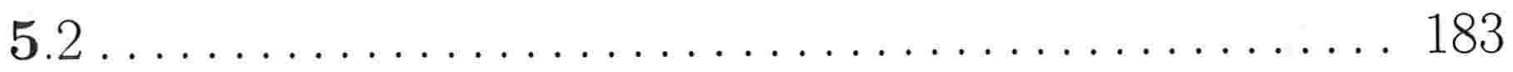


238

$5.2 .1 \ldots \ldots \ldots \ldots \ldots \ldots \ldots \ldots \ldots \ldots \ldots$

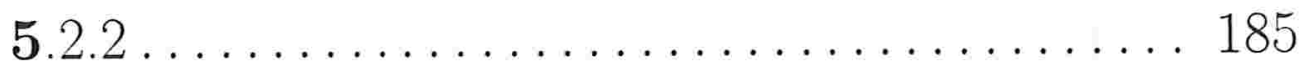

$5.2 .3 \ldots \ldots \ldots \ldots \ldots \ldots \ldots \ldots \ldots \ldots \ldots$

$5.2 .4 \ldots \ldots \ldots \ldots \ldots \ldots \ldots \ldots \ldots \ldots \ldots \ldots \ldots$

$5.2 .5 \ldots \ldots \ldots \ldots \ldots \ldots \ldots \ldots \ldots \ldots \ldots \ldots$

$5.2 .6 \ldots \ldots \ldots \ldots \ldots \ldots \ldots \ldots \ldots \ldots \ldots$

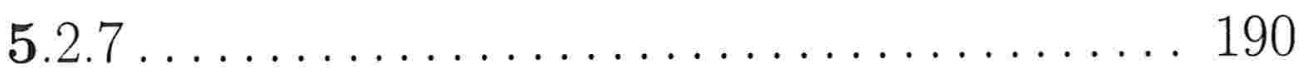

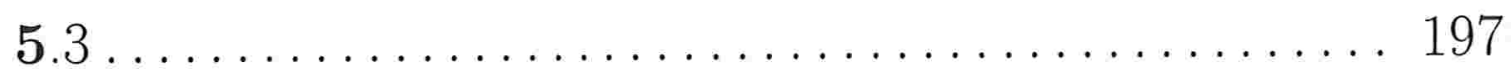

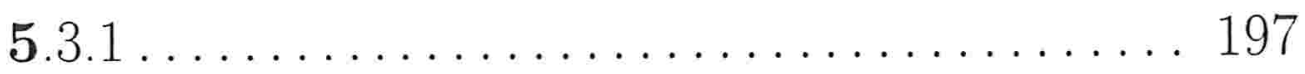

$5.3 .2 \ldots \ldots \ldots \ldots \ldots \ldots \ldots \ldots \ldots \ldots \ldots$

$5.3 .3 \ldots \ldots \ldots \ldots \ldots \ldots \ldots \ldots \ldots \ldots \ldots$

$5.3 .4 \ldots \ldots \ldots \ldots \ldots \ldots \ldots \ldots \ldots \ldots \ldots$

$5.3 .5 \ldots \ldots \ldots \ldots \ldots \ldots \ldots \ldots \ldots \ldots \ldots$

$5.3 .6 \ldots \ldots \ldots \ldots \ldots \ldots \ldots \ldots \ldots \ldots \ldots$ 
Acoplamentos e Associação

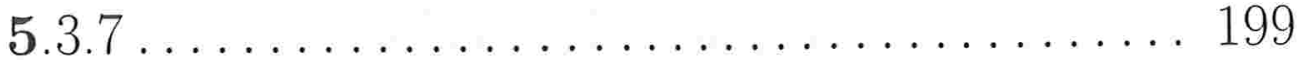

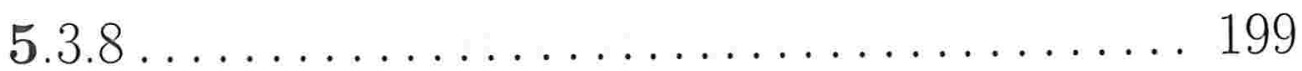

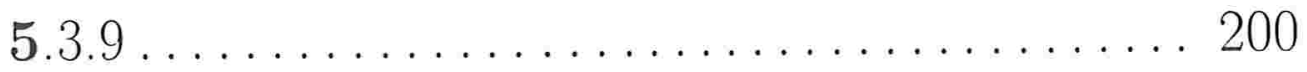




\section{Indice de Siglas}

Sigla Significado Tópico

$C D S$ _ Condicionalmente decrescente em seqüência _ 1.2.16 CIS _ Condicionalmente crescente em seqüência _ 1.2.16 f.d.a. Função de distribuição acumulada

f.d.p. —_ Função de distribuição de probabilidade

LTD _ Decrescente pela cauda esquerda 1.2 .18

LTI Crescente pela cauda esquerda 1.2 .18

$M T P_{2} \_$Multivariate totally positive of order 2 1.2 .3

$M R R_{2} \_$Multivariate reverse rule of order 2 1.2 .3 $N A$ Negativamente associado

$N D S$ Negativamente dependente através da ordem estocastica $\mathbf{1 . 2 . 1 5}$

NLOD _ _ Negative lower orthant dependence 1.2 .11 
NOD __ Negative orthant dependence 1.2 .12

$N Q D$ Negative quadrant dependent

NUOD __ Negative upper orthant dependence

$P A$ Positivamente associado

$P D S$ Positivamente dependente através da ordem estocastica 1.2 .14 $P F_{2}$ Frequência Polya de ordem 2 1.2.4 PLOD _ _ Positive lower orthant dependence 1.2 .11 POD __ Positive orthant dependence 1.2 .12 $P Q D \_$Positive quadrant dependent 1.2 .10 PUOD __ Positive upper orthant dependence 1.2 .11 $R R_{2} \_$Reverse rule of order 2 1.2 .4 $R R_{r} \_$Sinal-regra reversa de ordem $r$ 1.2 .21 $R T D \_$Decrescente pela cauda direita 
$R T I$ Crescente pela cauda direita 1.2 .17

$S D \_$Estocasticamente decrescente

1.2 .13

SI _ Estocasticamente crescente 1.2 .13

$S-M R R_{2}$ Fortemente $M R R_{2}$ 1.2.6

$S R_{r}$ Sinal-regular de ordem $r$

$T P_{2}$ $M T P_{2}$ caso bivariado 1.2.4 $\underline{X}$ vetor aleatório

$f_{\underline{X}}$ f.d.p. de $\underline{X}$

$F_{\underline{X}}$ f.d.a. de $\underline{X}$

$C_{\underline{X}} \longrightarrow$ Acoplamento associado a $F_{\underline{X}}$

1.2 .22

$\tau_{X_{1}, X_{2}} \longrightarrow$ Medida $\tau$ de Kendall entre $X_{1}$ e $X_{2}$ 1.2 .23

$>_{T P_{2}}$ ordem $T P_{2}$ 1.2.7 